\title{
Uniform regularity for the free surface compressible Navier-Stokes equations with or without surface tension
}

\author{
Yu Mei*, Yong Wang ${ }^{\dagger}, \quad$ Zhouping Xin ${ }^{\ddagger}$ \\ ${ }^{\dagger}$ Institute of Applied Mathematics, AMSS, CAS, Beijing 100190, China \\ ${ }^{\ddagger}$ Institute of Mathematical Science, The Chinese University of Hong Kong, Shatin, Hong Kong
}

\begin{abstract}
In this paper, we investigate the uniform regularity of solutions to the 3-dimensional isentropic compressible Navier-Stokes system with free surfaces and study the corresponding asymptotic limits of such solutions to that of the compressible Euler system for vanishing viscosity and surface tension. It is shown that there exists an unique strong solution to the free boundary problem for the compressible Navier-Stokes system in a finite time interval which is independent of the viscosity and the surface tension. The solution is uniform bounded both in $W^{1, \infty}$ and a conormal Sobolev space. It is also shown that the boundary layer for the density is weaker than the one for the velocity field. Based on such uniform estimates, the asymptotic limits to the free boundary problem for the ideal compressible Euler system with or without surface tension as both the viscosity and the surface tension tend to zero, are established by a strong convergence argument.
\end{abstract}

Keywords: Compressible Navier-Stokes, vanishing viscosity limit, free surface.

AMS: 35Q35, 35B65, 76N10

\section{Contents}

1 Introduction 2

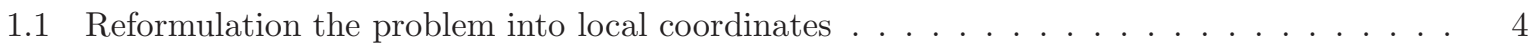

1.2 Main results . . . . . . . . . . . . . . . . . . . . . . . . . 7

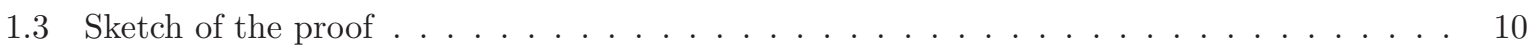

2 Preliminaries 14

2.1 General Inequalities . . . . . . . . . . . . . . . . . . . . . . . . 14

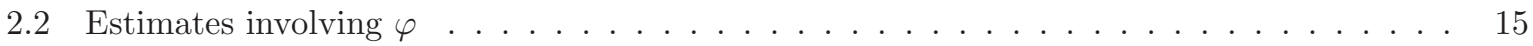

3 Equations for higher order conormal derivative terms 17

3.1 A commutator estimate . . . . . . . . . . . . . . . . . 17

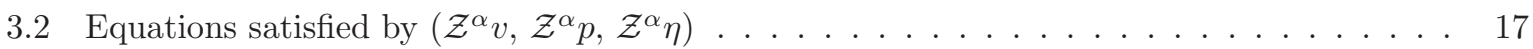

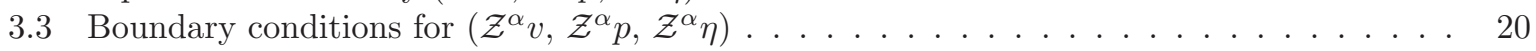

4 A priori Estimates 21

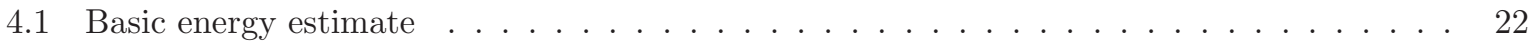

4.2 Higher order conormal energy estimates . . . . . . . . . . . . . . . . . 23

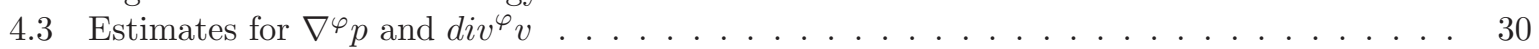

4.4 Normal derivative estimates: Part I . . . . . . . . . . . . . . . . . . . . . . . . . . 39

4.5 Normal derivative estimates: Part II . . . . . . . . . . . . . . . . . . 44

${ }^{*}$ Corresponding author.

Email addresses: $\quad$ ymei@math.cuhk.edu.hk(Yu Mei), $\quad$ yongwang@amss.ac.cn(Yong $\quad$ Wang), zpxin@ims.cuhk.edu.hk(Zhouping Xin) 


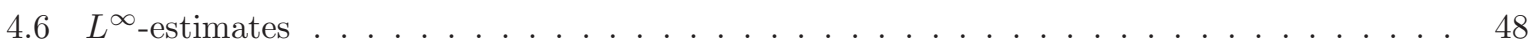

4.7 Proof of Theorem $4.1 \ldots \ldots \ldots \ldots \ldots \ldots \ldots$

5 Proof of the Main Results $\quad 60$

6 Appendix

\section{Introduction}

Consider the motion of a viscous isentropic compressible fluid with surface tension effect along a free boundary. It is governed by the following isentropic compressible Navier-Stokes system written in Eulerian coordinate as

$$
\left\{\begin{array}{l}
\rho_{t}^{\varepsilon}+\operatorname{div}\left(\rho^{\varepsilon} u^{\varepsilon}\right)=0 \\
\left(\rho^{\varepsilon} u^{\varepsilon}\right)_{t}+\operatorname{div}\left(\rho^{\varepsilon} u^{\varepsilon} \otimes u^{\varepsilon}\right)+\nabla p^{\varepsilon}=\mu \varepsilon \Delta u^{\varepsilon}+(\mu+\lambda) \varepsilon \nabla \operatorname{div} u^{\varepsilon},
\end{array} \quad x \in \Omega_{t}, t>0,\right.
$$

where $\Omega_{t}$ is a simply connected domain in $\mathbb{R}^{3}$ occupied by the fluid at time $t \geq 0, \rho^{\varepsilon}, u^{\varepsilon}$, which are unknowns, represent the density and the velocity field respectively. The pressure $p^{\varepsilon}$ is given by the $\gamma$-law

$$
p^{\varepsilon}=\left(\rho^{\varepsilon}\right)^{\gamma}, \quad \gamma>1
$$

The constant viscosity coefficients $\mu \varepsilon, \lambda \varepsilon$ satisfy the physical restrictions

$$
\mu>0,2 \mu+3 \lambda>0
$$

where the parameter $\varepsilon>0$ is the inverse of the Reynolds number. We assume the boundary of $\Omega_{t}$ is given by

$$
\Sigma_{t}=\left\{x \in \mathbb{R}^{3} \mid F^{\varepsilon}(x, t)=0\right\},
$$

where $F^{\varepsilon}(x, t)$ is an unknown function which will be uniquely determined by the velocity field. To study the well-posedness theory of this free boundary problem, the following two boundary conditions are imposed on $\Sigma_{t}, t>0$. On the one hand, the kinetic boundary condition, which states the fluid particles do not cross the free boundary, reads

$$
\partial_{t} F^{\varepsilon}+u^{\varepsilon} \cdot \nabla_{x} F^{\varepsilon}=0, \text { for } x \in \Sigma_{t} .
$$

On the other hand, we also need the dynamic boundary condition to balance the stress tensor on the both side of the free boundary. When the surface tension is taken into consideration, this boundary condition can be written as

$$
p^{\varepsilon} \mathbf{n}^{\varepsilon}=\left(2 \mu \varepsilon S u^{\varepsilon}+\lambda \varepsilon \operatorname{div} u^{\varepsilon}\right) \mathbf{n}^{\varepsilon}+p_{e} \mathbf{n}^{\varepsilon}-\sigma H^{\varepsilon} \mathbf{n}^{\varepsilon}, \quad x \in \Sigma_{t}
$$

which describes the stress tensor of the fluid is proportional to the mean curvature of the free boundary $\Sigma_{t}$. Here $S u^{\varepsilon}=\frac{1}{2}\left(\nabla u^{\varepsilon}+\nabla^{t} u^{\varepsilon}\right)$ and $\mathbf{n}^{\varepsilon}=\frac{\nabla_{x} F^{\varepsilon}}{\mid \nabla_{x} F^{\varepsilon}}$ denotes the outward normal vector of $\Omega_{t}, p_{e}$ is a given constant external pressure, $\sigma$ denotes the surface tension coefficient, $H$ is the double mean curvature of $\Sigma_{t}$ which can be expressed in the form

$$
H^{\varepsilon} \mathbf{n}^{\varepsilon}=\Delta_{\Sigma_{t}}(t) x, x=\left(x^{1}, x^{2}, x^{3}\right),
$$

where $\Delta_{\Sigma_{t}}(t)$ is the Laplace-Beltrami operator on $\Sigma_{t}$. We also impose the initial data for the compressible Navier-Stokes equations (1.1) as

$$
\left(\rho^{\varepsilon}, u^{\varepsilon}, F^{\varepsilon}\right)(0, x)=\left(\rho_{0}^{\varepsilon}, u_{0}^{\varepsilon}, F_{0}^{\varepsilon}\right)(x), \quad x \in \Omega_{0}
$$

such that

$$
0<\frac{1}{C_{0}} \leq \rho_{0}^{\varepsilon} \leq C_{0}<\infty
$$

where $\Omega_{0}$ is a given initial domain determined by $F_{0}^{\varepsilon}$ and $C_{0}$ is a given constant independent of $\varepsilon$.

The study of fluid motions with free surfaces is an important topic in fluid dynamics. For incompressible viscous fluids, we refer to the works by Beale [4, Tani [49, Solonnikov [43] and Guo-Tice [20] and references therein for the local well-posedness with or without surface tension. As to compressible 
viscous fluids, the local-wellposedness theory is established by Secchi-Valli [40] without surface tension, and by Solonnikov-Tani [44, Zajaczkowski 63, 64, and Tanaka-Tani 47] with surface tension and references therein. For the inviscid fluid, it is much more difficult to get the regularity of free boundary. $\mathrm{Wu}$ [54, 55] made a big breakthrough for the local well-posedness of irrotational incompressible Euler system in two and three dimensional, we also refer to [5, 11, 24, 34, 39] and the references therein for related works. Later, $\mathrm{Wu}$ [56] proved an almost global existence result in the 2-D case and the global existence results in 3-D case is proved by Wu 57 and Germain-Masmoudi-Shatah 17, 18. We also refer to [12, 14, 25, 41, 65] and the references therein. For the compressible Euler system, Lindblad [26] proved a local well-posedness result by using Lagrangian coordinates and Nash-Moser construction. Using the theory of symmetric hyperbolic systems, Trakhinin [50] provided a different proof for the local existence of solutions. Both the estimates in [26] and [50, had derivatives loss. Recently, Coutand-Hole-Shkoller 13 proved the well-posedness for the motion of a compressible liquid with or without surface tension, and with no derivative loss. The zero surface tension limit is also established in 13 .

Another classical and interesting problem in the mathematical theory of fluid mechanics is to study the asymptotic limit of the solutions to the Navier-Stokes equation at high Reynold number which corresponds to small viscosity. There has lots of literature on this problem when the domain has no boundaries, see for instances [10, 9, 22, 30. However, in the presence of physical boundaries, the problems become much more complicated. When no-slip boundary condition is imposed on the incompressible fluid in a fixed domain, the vanishing viscosity limit of the incompressible Navier-Stokes is one of the major open problems due to the possible appearance of boundary layers, as illustrated by Prandtl's theory. In [37, 38, the authors proved the (local in time) convergence of the incompressible Navier-Stokes flows to the Euler flows outside the boundary layer and to the prandtl flows in the boundary layer at the inviscid limit for the analytic initial data. Recently, Y. Maekawa [27] proved this limit when the initial vorticity is located away from the boundary in 2-D half plane. While, for the incompressible Navier-Stokes system with Navier-slip boundary condition in a fixed domain, considerable progress has been made on this problem. Indeed, the uniform $H^{3}$ bound and a uniform existence time interval as $\varepsilon$ tends to zero are obtained by Xiao-Xin in [58] for flat boundaries, which are generalized to $W^{k, p}$ in [6. 7]. However, such results can not be expected for general curved boundaries since boundary layer may appear due to non-trivial curvature as pointed out in 21. In such a case, Iftimie and Sueur have proved the convergence of the viscous solutions to the inviscid Euler solutions in $L^{\infty}\left(0, T, ; L^{2}\right)$-space by a careful construction of boundary layer expansions and energy estimates. However, to identify precisely the asymptotic structure and get the convergence in stronger norms such as $L^{\infty}\left(0, T ; H^{s}\right)(s>0)$, further a priori estimates and analysis are needed. Recently, Masmoudi-Rousset [31] established conormal uniform estimates for 3-D general smooth domains with the Naiver-slip boundary condition, which, in particular, implies the uniform boundedness of the normal first order derivatives of the velocity field. This allows the authors to obtain the convergence of the viscous solutions to the inviscid ones by a compact argument. Based on the uniform estimates in [31, better convergence with rates have been studied in [15] and [59]. In particular, Xiao-Xin [59] has proved the convergence in $L^{\infty}\left(0, T ; H^{1}\right)$ with an rate of convergence.

For the compressible Navier-Stokes equations in fixed domain, Xin-Yanagisawa 61 studied the vanishing viscosity limit of the linearized compressible Navier-Stokes system with the no-slip boundary condition in the 2-D half plane. Recently, Wang-Williams [51] constructed a boundary layer solution of the compressible Navier-Stokes equations with Navier-slip boundary conditions in 2-D half plane. The layers constructed in [51] are of width $O(\sqrt{\varepsilon})$ as the Prandtl boundary layer, but are of amplitude $O(\sqrt{\varepsilon})$ which is similar to the one 21] for the incompressible case. So, in general, it is impossible to obtain the $H^{3}$ or $W^{2, p}(p>3)$ estimates for the compressible Navier-Stokes system (1.1) with the generalized Navier-slip boundary condition. Later, Paddick 35] obtained an existence and conormal Sobolev regularity of strong solutions to the 3-D compressible isentropic Navier-Stokes system on the half-space with a Navier boundary condition. Recently, Wang-Xin-Yong [53] also obtained an uniform regularity for the solutions of the compressible Navier-Stokes with general Navier-slip boundary conditions in 3-D domains with curvature, especially, the vanishing viscosity limit of viscous solution to the corresponding inviscid one was also obtained with rate of convergence in $L^{\infty}$. In [53], it is also shown that the boundary layer for density is weaker than the one for velocity fields.

As to the vanishing viscosity limit problem of fluid motion with free surfaces, an interesting result was achieved recently, by Masmoudi-Rousset [32, that the local existence of solutions to the incompressible Navier-Stokes system with the gravity field but without surface tension in an uniform in $\varepsilon$ time interval by using a suitable functional framework based on conormal Sobolev spaces which minimizes the needed amount of normal regularity but which gives a control of the Lipschitz norm of the solution. These 
regularities of solution are necessary and reasonable for the free boundary problem. Actually, the Lipschitz regularity guarantee that the free surface moves along the partical path into outside of the fluid. And, in the vicinity of the free boundary, the expected behavior of the solution to the incompressible NavierStokes equation is $u^{\varepsilon}(x, t) \sim u(x, t)+\sqrt{\varepsilon} U\left(t, y, \frac{z}{\sqrt{\varepsilon}}\right)$ so that it is hard to obtain an uniform $H^{k}$-norm $(k \geq$ 2) estimate for $u^{\varepsilon}$ in a time interval independent of $\varepsilon$. In particular, a new existence result for the incompressible Euler equations can be obtained by strong convergence argument for the Navier-Stokes equations. Later, Elgindi-Lee has tried to study the similar problem in the presence of the surface tension in [16]. It should be noted that in the case surface tension be a positive constant, the pressure term in the Euler system becomes less regular. On the other hand, it is also interesting to investigate the zero surface tension limit for free boundary problems. This is nontrivial since that the surface tension coefficient $\sigma$ is connected to the mean curvature of free surface which is a second order derivative term of the boundary function. It is a subtle issue to obtain the uniform estimate in $\sigma$. Ambrose-Masmoudi [2, 3] studied the zero surface tension limit of irrotational water waves. The zero surface tension limit of incompressible Navier-Stokes equation has been established by Tan-Wang in 46 for small initial data. For the zero surface tension limit of compressible Euler with free surfaces, we can refer Coutand-Hole-Shkoller [13.

In this paper, we are interested in the existence of strong solution to the compressible Navier-Stokes system (1.1) with free surfaces in a finite interval of time independent of viscosity $\varepsilon$ and the surface tension $\sigma$ and the corresponding asymptotic limits problem of vanishing viscosity and zero surface tension. Formally, when the $\varepsilon$ tends to zero, the limit of (1.1) is compressible Euler system with surface tension

$$
\left\{\begin{array}{l}
\partial_{t} \rho+\operatorname{div}(\rho u)=0, \\
\rho \partial_{t} u+\rho(u \cdot \nabla) u+\nabla p=0,
\end{array} \quad x \in \Omega_{t}\right.
$$

with the boundary conditions

$$
\partial_{t} F+u \cdot \nabla F=0, \quad \text { and } p=p_{e}-\sigma H, \quad x \in \Sigma_{t}
$$

Moreover, when the surface tension coefficient $\sigma$ also goes to zero, it can be reduce to the compressible Euler system (1.10) with the boundary conditions

$$
\partial_{t} F+u \cdot \nabla F=0, \quad \text { and } p=p_{e}, \quad x \in \Sigma_{t},
$$

Motivated by Masmoudi-Rosset[32, we aim to obtain the uniform in $\varepsilon$ and $\sigma$ regularity in the anisotropic conormal Sobolev spaces and a control of the Lipschitz norm for solutions to the compressible NavierStokes equations (1.1) with free surfaces. Based on this uniform regularity, we can study the asymptotic limits to the ideal compressible Euler system with free surfaces as both the viscosity and the surface tension tend to zero by a strong compactness argument. Of course, the Taylor sign condition for the pressure on the boundary is needed to have local well-posedness for the Euler system. Usually, one expects that the boundary layer for density is weaker than the one for the velocity fields, we also aim to prove this facts in this paper. It should be mentioned that around same time as this research project, similar theory has been just established by Wang-Xin [52] for the incompressible fluids with rigid bottom below. Although there are some common difficulties caused by the appearance of surface tension for both cases, yet, as we shall explain later, there are major differences in these two cases. Different techniques are needed in the compressible case due to the lack of divergence free conditions which are essential in [32, 52.

\subsection{Reformulation the problem into local coordinates}

Due to the possible appearance of boundary layers, we shall consider this problem in the conormal Sobolev space as 32 . In order to define the conormal derivatives for the free boundary problem, we first assume that the initial domain $\Omega_{0}$ has a covering such that

$$
\Omega_{0} \Subset \tilde{\Omega}_{0} \cup_{k=1}^{n} \tilde{\Omega}_{k}
$$

with each $\tilde{\Omega}_{k}, k=0, \cdots, n$ being a convex domain. Here, $\tilde{\Omega}_{0} \Subset \Omega_{0}$ is chosen to satisfy

$$
\operatorname{dist}\left(\Sigma_{0}, \tilde{\Omega}_{0}\right)=2 d_{0}>0 .
$$


where $\Sigma_{0}$ is the boundary of $\Omega_{0}$ and $d_{0}$ is a given positive constant. For each fixed $k$ and $a>0$, define $\tilde{\Omega}_{k, a}$ as

$$
\tilde{\Omega}_{k, a}=\left\{x \in \mathbb{R}^{3} \mid \operatorname{dist}\left(x, \tilde{\Omega}_{k}\right)<a d_{0}\right\}
$$

Clearly,

$$
\Omega_{0} \Subset \tilde{\Omega}_{0,1} \cup_{k=1}^{n} \tilde{\Omega}_{k, 1} .
$$

$\tilde{\Omega}_{k, a}, k=1, \cdots, n$ will be called the boundary covering. Without loss of generality, we assume that

$$
\Omega_{0} \cap \tilde{\Omega}_{k, 1}=\left\{x \mid x_{3}<\tilde{h}_{k}^{\varepsilon}\left(x_{1}, x_{2}\right)\right\} \cap \tilde{\Omega}_{k, 1}, \quad \partial \Omega_{0} \cap \tilde{\Omega}_{k, 1}=\left\{x \mid x_{3}=\tilde{h}_{k}^{\varepsilon}\left(x_{1}, x_{2}\right)\right\} \cap \tilde{\Omega}_{k, 1},
$$

since the other cases can be handled similarly. Define

$$
\Sigma^{k, a}=\left\{\left(x_{1}, x_{2}\right) \mid x \in \tilde{\Omega}_{k, a} \cap \Omega_{0}, \text { for some } x=\left(x_{1}, x_{2}, x_{3}\right) \in \mathbb{R}^{3}\right\}, \quad k=1, \cdots, n .
$$

Then, $\Sigma^{k, a}$ is the projection of $\tilde{\Omega}_{k, a} \cap \Omega_{0}$ onto the hyperplane $\mathbb{R}_{x_{1}} \times \mathbb{R}_{x_{2}}$.

Similarly, for $\Omega_{t}$, we can define $\tilde{h}_{k}^{\varepsilon}\left(t, x_{1}, x_{2}\right)$ and $\Sigma_{t}^{k, a}$.

Remark 1.1 Under the assumption of the velocity is bounded, it is easy to know that $\tilde{\Omega}_{0,1}, \tilde{\Omega}_{k, \frac{1}{8}}, k=$ $1, \cdots, n$ is still an effective covering of $\Omega_{t}$ at least in a short time $t \in\left[0, T_{0}\right]$, i.e.

$$
\Omega_{t} \Subset \tilde{\Omega}_{0,1} \cup_{k=1}^{n} \tilde{\Omega}_{k, \frac{1}{8}}, \quad \forall t \in\left[0, T_{0}\right], \text { with } T_{0} \ll 1,
$$

where $T_{0}$ depends on the upper bound of velocity and $d_{0}$.

As in 32, to deal with the free boundary problem, one can reduce the problem in each boundary covering into a fixed domain and use the local coordinate to define the conormal derivatives. Instead of using Lagrangian variables, we define a family of differmorphism $\Phi_{k}^{\varepsilon}(t, \cdot)$ by the following process. Since $\tilde{h}_{k}^{\varepsilon}$ is defined locally, we first extend $\tilde{h}_{k}^{\varepsilon}$ to $\mathbb{R}^{2}$ by multiplying a cut-off function

$$
h_{k}^{\varepsilon}(t, y)=\tilde{\psi}_{k}(y) \tilde{h}_{k}^{\varepsilon}(t, y) \text {, }
$$

where $\tilde{\psi}_{k}(y)$ is a smooth function satisfying

$$
\tilde{\psi}_{k}(y)= \begin{cases}1, & y \in \Sigma_{k, \frac{3}{4}}(0), \\ 0, & y \in \Sigma_{k, \frac{7}{8}}(0) .\end{cases}
$$

Then, define $\eta_{k}^{\varepsilon}$ as

$$
\hat{\eta}_{k}^{\varepsilon}(t, \xi, z)=\kappa(z\langle\xi\rangle) \hat{h}_{k}^{\varepsilon}(t, \xi)
$$

where $\hat{\imath}$ denotes the Fourier transform with respect to $y$ and $\kappa$ is defined by

$$
\kappa(z\langle\xi\rangle)=e^{-z^{2}\langle\xi\rangle^{2}}=e^{-z^{2}\left(1+|\xi|^{2}\right)},
$$

which implies that

$$
\eta_{k}^{\varepsilon}(t, y, z)=\frac{1}{2} e^{-z^{2}} \int_{\mathbb{R}^{2}} \frac{1}{z^{2}} e^{\frac{|y-\tilde{y}|^{2}}{4 z^{2}}} h_{k}^{\varepsilon}(t, \tilde{y}) d \tilde{y} .
$$

As in $\left[32, \varphi_{k}^{\varepsilon}\right.$ can be chosen as

$$
\varphi_{k}^{\varepsilon}(t, y, z)=A z+\eta_{k}^{\varepsilon}(t, y, z),
$$

where $A>0$ is a constant chosen such that

$$
\partial_{z} \varphi_{k}^{\varepsilon}(0, y, z) \geq 1,(y, z) \in \mathcal{S} .
$$

Therefore, the differmorphism $\Phi_{k}^{\varepsilon}$ can be defined by

$$
\begin{gathered}
\Phi_{k}^{\varepsilon}(t, \cdot): \mathcal{S}=\mathbb{R}^{2} \times(-\infty, 0) \rightarrow D_{k}(t) \supset \Omega_{t} \cap \tilde{\Omega}_{k, \frac{5}{8}} \\
(y, z) \mapsto x:=\left(y, \varphi_{k}^{\varepsilon}(t, y, z)\right),
\end{gathered}
$$

where $D_{k}(t)$ is defined by

$$
D_{k}(t):=\left\{x=\left(y, x_{3}\right) \mid y \in \mathbb{R}^{2}, x_{3}<h_{k}^{\varepsilon}(t, y)\right\} .
$$


By the above parameterizations, the free surface becomes $z=0$ locally. Although we have defined an extension of $\tilde{h}_{k}^{\varepsilon}$ in $\mathbb{R}^{2} \times(-\infty, 0)$, but it will be more convenient to work in a vicinity of the boundary, i.e.

$$
\mathcal{S}_{k, a}(t)=\left\{(y, z) \mid(y, z) \in \mathbb{R}^{3} \text { such that } x=\left(y, \varphi_{k}^{\varepsilon}(t, y, z)\right) \in \tilde{\Omega}_{k, a}\right\} \subset \mathcal{S}, \quad k=1, \cdots, n .
$$

with $0 \leq a \leq \frac{3}{4}$. Since the velocity will be uniform bounded, one has that

$$
\mathcal{S}_{k, \frac{1}{8}}(t) \subset \mathcal{S}_{k, \frac{1}{4}}(0) \subset \mathcal{S}_{k, \frac{3}{8}}(t) \subset \mathcal{S}_{k, \frac{1}{2}}(0) \subset \mathcal{S}_{k, \frac{5}{8}}(t) \subset \mathcal{S}_{k, \frac{3}{4}}(0), \quad \forall t \in\left[0, T_{0}\right], \quad T_{0} \ll 1
$$

Since $\Sigma_{t}$ is given locally by $x_{3}=h(t, y)$ (Henceforth the subscript $k$ and the supscript $\varepsilon$ in $\rho^{\varepsilon}, u^{\varepsilon}, h_{k}^{\varepsilon}$ will be omitted for notational convenience), it is convenient to use the local coordinates $(y, z)$ defined in (1.24) at least locally. Once the choice of $\varphi$ is made, based on the observation of (1.27), one can reduce locally (neighborhood near the boundary $\Sigma_{t}$ ) the problem into the fixed domain $\mathcal{S}_{\frac{1}{2}}(0)$ by setting

$$
\varrho(t, y, z)=\rho(t, \Phi(t, y, z)), \quad v(t, y, z)=u(t, \Phi(t, y, z)), \quad(y, z) \in \mathcal{S}_{\frac{1}{2}}(0) .
$$

For simplicity, we will denote also $(\rho, u)$ as $(\varrho, v)$ in the interior domain.

Now, we rewrite the system (1.1) to the one for $(\varrho, v, h)$ in the new coordinate $(y, z)$ of the fixed domain $\mathcal{S}_{\frac{1}{2}}(0)$. First, one introduces the operators $\partial_{i}^{\varphi}, i=t, 1,2,3$ such that

$$
\partial_{i}^{\varphi} \varrho=\left(\partial_{i} \rho\right) \circ(t, \Phi(t, \cdot)), \quad \partial_{i}^{\varphi} v=\left(\partial_{i} u\right) \circ(t, \Phi(t, \cdot)),
$$

which directly yields that

$$
\left\{\begin{array}{l}
\partial_{i}^{\varphi}=\partial_{i}-\frac{\partial_{i} \varphi}{\partial_{z} \varphi} \partial_{z}, i=t, 1,2 \\
\partial_{3}^{\varphi}=\frac{1}{\partial_{z} \varphi} \partial_{z}
\end{array}\right.
$$

Then, the compressible Navier-Stokes system (1.1) is locally reduced to the system for $(\varrho, v)$ as

$$
\left\{\begin{array}{l}
\partial_{t}^{\varphi} \varrho+\operatorname{div}^{\varphi}(\varrho v)=0 \\
\varrho \partial_{t}^{\varphi} v+\varrho v \cdot \nabla^{\varphi} v+\nabla^{\varphi} p(\varrho)=2 \mu \varepsilon \operatorname{div}^{\varphi}\left(S^{\varphi} v\right)+\lambda \varepsilon \nabla^{\varphi} \operatorname{div}^{\varphi} v, \quad(y, z) \in \mathcal{S}_{\frac{1}{2}}(0), t>0
\end{array}\right.
$$

where $\operatorname{div}^{\varphi} v=\sum_{i=1}^{3} \partial_{i}^{\varphi} v_{i}, \nabla^{\varphi}=\left(\partial_{1}^{\varphi}, \partial_{2}^{\varphi}, \partial_{3}^{\varphi}\right)^{t}, v \cdot \nabla^{\varphi}=\sum_{i=1}^{3} v_{i} \partial_{i}^{\varphi}$ and $S^{\varphi} v=\frac{1}{2}\left(\nabla^{\varphi} v+\nabla^{\varphi} v^{t}\right)$. And the two boundary conditions read as

$$
\partial_{t} h=v \cdot \mathbf{N}=-v_{y}(t, y, 0) \cdot \nabla_{y} h+v_{3}(t, y, 0),(y, z) \in \mathcal{S}_{\frac{1}{2}}(0)
$$

and

$$
p \mathbf{N}=\left(2 \mu \varepsilon S^{\varphi} v+\lambda \varepsilon \operatorname{div}^{\varphi} v\right) \mathbf{N}+p_{e} \mathbf{N}-\sigma \nabla_{y} \cdot \frac{\nabla_{y} h}{\sqrt{1+\left|\nabla_{y} h\right|^{2}}} \mathbf{N}, \quad(y, 0) \in \mathcal{S}_{\frac{1}{2}}(0),
$$

where $v=\left(v_{y}, v_{3}\right)^{t}:=\left(v_{1}, v_{2}, v_{3}\right)^{t}, \nabla=\left(\nabla_{y}, \partial_{z}\right)^{t}:=\left(\partial_{1}, \partial_{2}, \partial_{z}\right)^{t}$ and $\mathbf{N}=\left(-\partial_{1} h,-\partial_{2} h, 1\right)^{t}$. For later use, we define $\mathbf{n}=\frac{\mathbf{N}}{|\mathbf{N}|}$.

Remark 1.2 By using the covering of $\Omega_{t}$, one can always assume that each vector field $(\varrho, v, h)$ is supported in either $\mathcal{S}_{k, \frac{1}{2}}(0)$ in $(y, z)$ coordinate, or in $\tilde{\Omega}_{0,1}$, since this assumption can be achieved by multiplying a cut-off function $\psi_{k} \in C_{0}^{\infty}\left(\mathbb{R}_{+}^{3}\right)$ such that $\psi_{k} \equiv 1,(y, z) \in \mathcal{S}_{k, \frac{1}{4}}(0)$ and $\psi_{k} \equiv 0,(y, z) \in$ $\mathbb{R}_{+}^{3}-\mathcal{S}_{k, \frac{1}{2}}(0)$, and $\psi_{0} \in C_{0}^{\infty}\left(\mathbb{R}^{3}\right)$ such that $\psi_{0}(x) \equiv 1$ for $x \in \tilde{\Omega}_{0, \frac{3}{4}}$ and $\psi_{0}(x) \equiv 0$ for $x \in \mathbb{R}^{3}-\tilde{\Omega}_{0,1}$, respectively. Therefore, using this localization arguments, one can also assume that the problem (1.31) holds in $\mathcal{S}$ or $\mathbb{R}^{3}$ and (1.32)-1.33) hold on $z=0, y \in \mathbb{R}^{2}$.

To measure the regularity of functions defined in $\mathcal{S}$, we shall use the Sobolev conormal spaces as in 32. Introduce the vector fields

$$
Z_{i}=\partial_{i}, i=1,2, \quad Z_{3}=\frac{z}{1-z} \partial_{z}
$$

The Sobolev conormal spaces $H_{c o}^{m}$ is defined as

$$
H_{c o}^{m}(\mathcal{S})=\left\{f \in L^{2}(\mathcal{S})\left|Z^{\alpha} f \in L^{2}(\mathcal{S}), \quad\right| \alpha \mid \leq m\right\},
$$


where $Z^{\alpha}=Z_{1}^{\alpha_{1}} Z_{2}^{\alpha_{2}} Z_{3}^{\alpha_{3}}$ with norm defined as

$$
\|f\|_{m}^{2}=\sum_{|\alpha| \leq m}\left\|Z^{\alpha} f\right\|^{2}
$$

Similarly, set

$$
W_{c o}^{m . \infty}(\mathcal{S})=\left\{f \in L^{\infty}(\mathcal{S})\left|Z^{\alpha} f \in L^{\infty}(\mathcal{S}), \quad\right| \alpha \mid \leq m\right\}
$$

and

$$
\|f\|_{m, \infty}=\sum_{|\alpha| \leq m}\left\|Z^{\alpha} f\right\|_{L^{\infty}} .
$$

However, for the compressible Navier-Stokes, the time derivative should be involved in the energy functional. So, we also set

$$
Z_{0}=\partial_{0}=\partial_{t}, \quad \mathcal{Z}^{\alpha}=Z_{0}^{\alpha_{0}} Z_{1}^{\alpha_{1}} Z_{2}^{\alpha_{2}} Z_{3}^{\alpha_{3}}
$$

and, for smooth space-time function $f(t, y, z)$ with $t \geq 0,(y, z) \in S$, set the following notations

$$
\begin{gathered}
\|f(t)\|_{\mathcal{H}^{m}}^{2}=\sum_{|\alpha| \leq m}\left\|\mathcal{Z}^{\alpha} f(t)\right\|^{2}, \quad\|f(t)\|_{\mathcal{H}^{k, \infty}}^{2}=\sum_{|\alpha| \leq k}\left\|\mathcal{Z}^{\alpha} f(t)\right\|_{L^{\infty}}^{2}, \\
\left\|\nabla \mathcal{Z}^{m} f(t)\right\|^{2}=\sum_{|\alpha| \leq m}\left\|\nabla \mathcal{Z}^{\alpha} f(t)\right\|^{2}
\end{gathered}
$$

and

$$
|f(t)|_{\mathcal{H}^{m}}^{2}=\sum_{|\alpha| \leq m}\left|\mathcal{Z}^{\alpha} f(t, \cdot, z=0)\right|_{L^{2}}^{2}, \quad\left|\mathcal{Z}^{m} f(t)\right|_{s}^{2}=\sum_{|\alpha| \leq m}\left|\mathcal{Z}^{\alpha} f(t, \cdot, z=0)\right|_{s}^{2}
$$

Remark 1.3 It should emphasized that the norms defined above can be used in each covering although we have not written this fact explicitly.

Remark 1.4 In the present paper, we will focus on the estimates near the boundary, i.e. in $\mathcal{S}_{\frac{1}{2}}(0)$. The interior estimate is easy to obtain in a similar way by a cut-off approach such that no boundary terms involves, since the conormal Sobolev space is equivalent to the standard Sobolev space in the interior domain. The global estimates follow from collecting all the local boundary ones and the interior one.

Notations: Throughout this paper, the positive generic constants that are independent of $\varepsilon, \sigma$ are denoted by $c, C$. And $\|\cdot\|$ denotes the standard $L^{2}$ norm, and $\|\cdot\|_{H^{m}}(m=1,2,3, \cdots)$ to denote the Sobolev $H^{m}$ norm. The notation $|\cdot|_{s}$ will be used for the standard Sobolev $H^{s}$ norm of functions defined on boundary, and note that this norm involves only tangential derivatives. $a \lesssim b$ used here denotes $a \leq C b$ for some positive constant independent of $\varepsilon, \sigma . \Lambda(\cdot)$ denotes a polynomial function independent of $\varepsilon$ and $\sigma$, and may change from line to line.

\subsection{Main results}

The aim of this paper is to get a local well-posedness result for strong solutions to (1.1) in an interval of time independent of $\varepsilon$ and $\sigma$ for $\varepsilon \in(0,1], \sigma \in[0,1]$. Note that such a result will also imply the local existence of strong solutions for the Euler equations with or without surface tension. As it is well-known [14, 32, 41, 55, in the absence of viscosity and surface tension, we need to require the initial pressure $p_{0}$ for the compressible Euler flow satisfies the Taylor sign condition (see, for example [45] and [36]), i.e.

$$
0<c_{0} \leq-\frac{\partial p_{0}^{\varepsilon}}{\partial \mathbf{N}}, \quad x \in \Sigma_{t}
$$

In the local coordinate $(y, z)$, the initial data (1.8) is reduced to

$$
\left(\varrho^{\varepsilon}, v^{\varepsilon}, h^{\varepsilon}\right)(y, z, 0)=\left(\varrho_{0}^{\varepsilon}, v_{0}^{\varepsilon}, h_{0}^{\varepsilon}\right)(y, z),
$$

such that

$$
0<\frac{1}{C_{0}} \leq \varrho_{0}^{\varepsilon} \leq C_{0}<\infty
$$


and the Taylor sign condition becomes

$$
0<c_{0} \leq-\partial_{z}^{\varphi} p_{0}^{\varepsilon}, \quad z=0 .
$$

Since boundary layers may appear in the vicinity of physical boundaries, in order to obtain uniform estimates for solutions to the compressible Navier-Stokes system with free surfaces, one needs to find a suitable functional space. Here, we define the functional space $X_{m}^{\varepsilon}(T)$ for $(p, v, h)=(p, v, h)(t)$ as follows:

$$
X_{m}^{\varepsilon}(T)=\left\{(p, v, h) \in L^{\infty}\left([0, T], L^{2}\right) ; \quad \operatorname{esssup}_{0 \leq t \leq T}\|(p, v, h)(t)\|_{X_{m}^{\varepsilon}}<+\infty\right\},
$$

where the norm $\|(\cdot, \cdot, \cdot)\|_{X_{m}^{\varepsilon}}$ is given by

$$
\begin{aligned}
\|(p, v, h)(t)\|_{X_{m}^{\varepsilon}}^{2}=\|(p, v)(t)\|_{\mathcal{H}^{m}}^{2}+\|\nabla(p, v)(t)\|_{\mathcal{H}^{m-2}}^{2}+\|\Delta p(t)\|_{\mathcal{H}^{1}}^{2}+\left|\left(h, \sqrt{\sigma} \nabla_{y} h\right)(t)\right|_{\mathcal{H}^{m}}^{2} \\
\quad+\|\nabla(p, v)(t)\|_{\mathcal{H}^{1, \infty}}^{2}+\varepsilon\|\nabla(p, v)(t)\|_{\mathcal{H}^{m-1}}^{2}+\varepsilon\|\Delta p(t)\|_{\mathcal{H}^{2}}^{2}+\varepsilon\left\|\nabla^{2} v(t)\right\|_{L^{\infty}}^{2}+\varepsilon\left|\mathcal{Z}^{m} h\right|_{\frac{1}{2}}^{2} .
\end{aligned}
$$

Note that the a priori estimates in Theorem 4.1 below is obtained in the case that the approximate solution is sufficient smooth up to the boundary. In order to obtain a selfcontained result, one needs to assume that the approximate initial data satisfies the boundary compatibility conditions. Therefore, we set

$$
\begin{aligned}
& X_{N S, a p}^{\varepsilon, m}=\{(\rho, u) \in C^{3 m}\left(\Omega_{0}\right), F\left(x\left(s_{1}, s_{2}\right)\right) \in C^{3 m}(U) \mid \text { The Taylor sign condition (1.38) holds; } \\
& \partial_{t}^{k} \rho, \partial_{t}^{k} u \text { and } \partial_{t}^{k} F, k=1, \cdots, m \text { are defined through the Navier-Stokes equations } \\
&\left(\underline{1.1)} \text { and (1.5) }, \text { respectively; } \partial_{t}^{k}(\rho, u, F), k=0,1, \cdots, m-1\right. \text { satisfy the } \\
&\text { boundary compatibility conditions (1.6) }\}
\end{aligned}
$$

where the domain $\Omega_{0}$ is defined through $F$ as previous. Note that the definition of $X_{N S, a p}^{\varepsilon, m}$ is given in the Euler coordinate, but it will be changed automatically into local coordinate when one evaluates it by some norm, and we should keep this in mind all the time. Define

$$
X_{N S}^{\varepsilon, m}=\text { The closure of } X_{N S, a p}^{\varepsilon, m} \text { in the norm }\|(\cdot, \cdot, \cdot)\|_{X_{m}^{\varepsilon}} .
$$

We assume that the initial data $\left(\varrho_{0}^{\varepsilon}, v_{0}^{\varepsilon}, h_{0}^{\varepsilon}\right) \in X_{N S}^{\varepsilon, m}$ and satisfies

$$
\sup _{\varepsilon \in(0,1], \sigma \in[0,1]}\left\{\left\|\left(p_{0}^{\varepsilon}, v_{0}^{\varepsilon}, h_{0}^{\varepsilon}\right)\right\|_{X_{m}^{\varepsilon}}^{2}+\left\|\nabla v_{0}^{\varepsilon}\right\|_{\mathcal{H}^{m-1}}^{2}\right\} \leq \tilde{C}_{0}
$$

where $p_{0}^{\varepsilon}=p\left(\varrho_{0}^{\varepsilon}\right), C_{0}>0, \tilde{C}_{0}>0$ are positive constants independent of $\varepsilon \in(0,1]$ and $\sigma \in[0,1]$. Thus, the initial data $\left(\varrho_{0}^{\varepsilon}, u_{0}^{\varepsilon}\right)$ is assumed to have higher space regularity and compatibilities. It should be noted that the initial data may also depends on $\sigma$, but, for simplicity, this will not be written explicitly.

Our main result is as follows:

Theorem 1.5 (Uniform Regularity) Assume that $m \geq 6, \varepsilon \in(0,1]$ and $\sigma \in[0,1]$. Consider the initial data $\left(\rho_{0}^{\varepsilon}, u_{0}^{\varepsilon}, F_{0}^{\varepsilon}\right) \in X_{N S}^{\varepsilon, m}$ given in (1.8) satisfying (1.40), (1.41) and (1.46). Then there exists a time $T_{0}>0$ and $\tilde{C}_{1}>0$ independent of $\varepsilon$ and $\sigma$, such that there exists an unique solution $\left(\rho^{\varepsilon}, u^{\varepsilon}, F^{\varepsilon}\right)$ to the free surface problem (1.1), (1.5) and (1.6) which is defined on $\left[0, T_{0}\right]$ and satisfies the following estimates:

$$
\begin{aligned}
\sup _{0 \leq t \leq T_{0}} & \left\{\left\|\left(p^{\varepsilon}, v^{\varepsilon}\right)(t)\right\|_{\mathcal{H}^{m}}^{2}+\left\|\nabla\left(p^{\varepsilon}, v^{\varepsilon}\right)(t)\right\|_{\mathcal{H}^{m-2}}^{2}+\left\|\Delta p^{\varepsilon}(t)\right\|_{\mathcal{H}^{1}}^{2}+\left|h^{\varepsilon}\right|_{\mathcal{H}^{m}}^{2}+\sigma\left|\nabla_{y} h^{\varepsilon}\right|_{\mathcal{H}^{m}}^{2}\right. \\
& \left.+\left\|\nabla\left(p^{\varepsilon}, v^{\varepsilon}\right)(t)\right\|_{\mathcal{H}^{1, \infty}}^{2}+\varepsilon\left\|\nabla\left(p^{\varepsilon}, v^{\varepsilon}\right)(t)\right\|_{\mathcal{H}^{m-1}}^{2}+\varepsilon\left\|\Delta p^{\varepsilon}(t)\right\|_{\mathcal{H}^{2}}^{2}+\varepsilon\left\|\nabla^{2} v^{\varepsilon}\right\|_{L^{\infty}}^{2}+\varepsilon\left|\mathcal{Z}^{m} h\right|_{\frac{1}{2}}^{2}\right\} \\
& +\int_{0}^{T_{0}}\left\|\nabla p^{\varepsilon}(t)\right\|_{\mathcal{H}^{m-1}}^{2}+\left\|\Delta p^{\varepsilon}(t)\right\|_{\mathcal{H}^{2}}^{2} d t+\int_{0}^{T_{0}}\left\|\nabla v^{\varepsilon}(t)\right\|_{\mathcal{H}^{m-1}}^{4} d t \\
& +\varepsilon \int_{0}^{T_{0}}\left\|\nabla v^{\varepsilon}(t)\right\|_{\mathcal{H}^{m}}^{2}+\left\|\nabla^{2} v^{\varepsilon}(t)\right\|_{\mathcal{H}^{m-2}}^{2} d t+\varepsilon^{2} \int_{0}^{T_{0}}\left\|\nabla^{2} v^{\varepsilon}(t)\right\|_{\mathcal{H}^{m-1}}^{2} d t \leq \tilde{C}_{1}
\end{aligned}
$$

and

$$
\frac{1}{2 C_{0}} \leq \varrho^{\varepsilon}(t) \leq 2 C_{0} \quad \forall t \in\left[0, T_{0}\right]
$$

where $\tilde{C}_{1}$ only depends on $C_{0}, \tilde{C}_{0}$ and $d_{0}$. 
Note that in the above result, time derivatives shall be involved in the energy functional. This is not necessary for incompressible flow without surface tension studied by Masmoudi-Rousset 32, since the pressure $p$ there solves an elliptic equation with a Dirichlet boundary condition so that elliptic regularity of $p$ in the spatial conormal spaces can be used to obtain the uniform estimates. But, as to the incompressible flow with surface tension studied by Wang-Xin [52, time derivatives are also needed since the Neumann boundary condition for $p$, which was derived from the momentum equation, shall be used. It should be noted that the main difficulties in 52 for the incompressible flow with surface tension are that there are no any estimates of the highest order time derivatives $\partial_{t}^{m} p$. However, the difficulties for the compressible fluid are different, since the pressure $p$ here satisfies a transport equation so that the regularity of $\partial_{t}^{m} p$ can be derived by an energy method. As will be seen later, the main difficulties here are to derive delicate estimates of $\operatorname{div}^{\varphi} v$ and uniform in $\sigma$ estimates (when $\sigma \neq 0$ ) of the boundary term as (4.2.15) involving surface tension. We also give some remarks on our uniform regularity in Theorem 1.5 as follows.

Remark 1.6 Note that $\left(\rho^{\varepsilon}, u^{\varepsilon}, F^{\varepsilon}\right)$ in Theorem 1.5 is the solution to (1.1) (1.5) and (1.6) given in Eulerian coordinate of $\Omega_{t}$. While, $\left(\varrho^{\varepsilon}, v^{\varepsilon}, h^{\varepsilon}\right)$ defined locally in the fixed domain $\mathcal{S}$ is used in all the a priori estimates. Since the solution has enough regularity, they can be changed to each other, equivalently. These two notations will be used without confusion throughout this paper.

Remark 1.7 The Taylor sign condition (1.38) or (1.41) is necessary when one studies both vanishing viscosity and zero surface tension limits to the ideal compressible Euler system. However, when surface tension is considered(for fixed $\sigma>0$ ), this is not necessary since the surface tension prevent the RayleighTaylor instability

Remark 1.8 One can construct a class of initial data to satisfy the compatibility conditions (1.44). e.g. Let $\Omega_{0}=B_{R_{0}}$ be a ball with radius $R_{0}$. When $\sigma>0$ is fixed, let $\left(\rho_{0}^{\varepsilon}, u_{0}^{\varepsilon}\right)$ be sufficiently smooth functions, and $\rho_{0}^{\varepsilon}$ be a positive constant $\rho_{0 c}$ and $u_{0}^{\varepsilon}$ vanishes in a vicinity of the boundary. By choosing $p\left(\rho_{0 c}\right)=$ $p_{e}+\frac{2 \sigma}{R_{0}}$, then it is obvious that $\left(\rho_{0}^{\varepsilon}, u_{0}^{\varepsilon}\right)$ satisfies the boundary compatibility conditions. When $\sigma=0$, it becomes a little complicated due to the Taylor sign condition. In this case, one can assume that the initial data is radial symmetric (near the boundary of ball), which are polynomial functions of radius $R_{0}-r$, so that the boundary compatibility conditions can be reduce to algebraic equations for coefficients of polynomials. We omit the details here for simplicity.

Remark 1.9 It follows from the uniform estimate of $\left\|\Delta p^{\varepsilon}(t)\right\|_{\mathcal{H}^{1}}^{2}$ that the boundary layer for the density $\rho^{\varepsilon}$ is weaker than the one for the velocity $u^{\varepsilon}$ as expected.

Based on the uniform estimates given in Theorem 1.5, we can justify the vanishing viscosity limit, zero surface tension limit and the local existence of solution to the free surface problem for the compressible Euler system.

Theorem 1.10 (Inviscid Limit) For any fixed $\sigma \geq 0$, under the assumptions in Theorem 1.5, and in addition that there exists $\left(\varrho_{0}, u_{0}, h_{0}\right)$ such that

$$
\lim _{\varepsilon \rightarrow 0}\left\|\left(\varrho_{0}^{\varepsilon}-\varrho_{0}, v_{0}^{\varepsilon}-v_{0}\right)\right\|_{L^{2}}+\left|h_{0}^{\varepsilon}-h_{0}\right|_{L^{2}}=0 .
$$

Then, there exists $(\rho, u, F)(x, t)$ on the time interval $\left[0, T_{0}\right]$, and satisfies

$$
\begin{gathered}
\sup _{0 \leq t \leq T_{0}}\left\{\|(p, v)(t)\|_{\mathcal{H}^{m}}^{2}+\|\nabla(p, v)(t)\|_{\mathcal{H}^{m-2}}^{2}+\left|\left(h, \sqrt{\sigma} \nabla_{y} h\right)(t)\right|_{\mathcal{H}^{m}}^{2}+\|\Delta p(t)\|_{\mathcal{H}^{1}}^{2}+\|\nabla(p, v)(t)\|_{\mathcal{H}^{1, \infty}}^{2}\right\} \\
+\int_{0}^{T_{0}}\|\nabla p(t)\|_{\mathcal{H}^{m-1}}^{2} d t+\int_{0}^{T_{0}}\|\Delta p(t)\|_{\mathcal{H}^{2}}^{2} d t+\int_{0}^{T_{0}}\|\nabla v(t)\|_{\mathcal{H}^{m-1}}^{4} d t \leq \tilde{C}_{1}<\infty \\
0<\frac{1}{2 C_{0}} \leq \rho(t) \leq 2 C_{0}<\infty, \quad \forall t \in\left[0, T_{0}\right]
\end{gathered}
$$

and

$$
\lim _{\varepsilon \rightarrow 0} \sup _{t \in\left[0, T_{0}\right]}\left\|\left(\varrho^{\varepsilon}-\varrho, v^{\varepsilon}-v\right)(t)\right\|_{L^{\infty}}+\left|h^{\varepsilon}-h\right|_{W^{1, \infty}}=0
$$


where $(\varrho, v, h)$ is the localized representation of $(\rho, u, F)$. Furthermore, $(\rho, u, F)$ is the unique solution to the free surface Euler equations

$$
\left\{\begin{array}{l}
\partial_{t} \rho+\operatorname{div}(\rho u)=0, \\
\rho \partial_{t} u+\rho(u \cdot \nabla) u+\nabla p=0,
\end{array} \quad x \in \Omega_{t}\right.
$$

with the boundary conditions

$$
\partial_{t} F+u \cdot \nabla F=0, \quad \text { and } p=p_{e}-\sigma H, \quad x \in \Sigma_{t},
$$

where $\Omega_{t}$ is the domain occupied by the fluid on time $t \geq 0$ and the boundary of $\Omega_{t}$ is given by

$$
\Sigma_{t}=\left\{x \in \mathbb{R}^{3} \mid F(x, t)=0\right\} .
$$

Similarly, by the strong compactness argument in Theorem 1.10, as $\varepsilon$ and $\sigma$ tend to zero independently, one can obtain that

Theorem 1.11 (Inviscid and Zero Surface Tension Limit) Under the assumptions of Theorem 1.5 , if we assume in addition that there exists $\left(\varrho_{0}, u_{0}, h_{0}\right)$ such that

$$
\lim _{\varepsilon \rightarrow 0, \sigma \rightarrow 0}\left\|\left(\varrho_{0}^{\varepsilon, \sigma}-\varrho_{0}, v_{0}^{\varepsilon, \sigma}-v_{0}\right)\right\|_{L^{2}}+\left|h_{0}^{\varepsilon, \sigma}-h_{0}\right|_{L^{2}}=0 .
$$

Then, there exists $(\rho, u, F)(t)$ on the time interval $\left[0, T_{0}\right]$, and satisfies

$$
\begin{gathered}
\sup _{0 \leq t \leq T_{0}}\left\{\|(p, v)(t)\|_{\mathcal{H}^{m}}^{2}+\|\nabla(p, v)(t)\|_{\mathcal{H}^{m-2}}^{2}+|h(t)|_{\mathcal{H}^{m}}^{2}+\|\Delta p(t)\|_{\mathcal{H}^{1}}^{2}+\|\nabla(p, v)(t)\|_{\mathcal{H}^{1, \infty}}^{2}\right\} \\
+\int_{0}^{T_{0}}\|\nabla p(t)\|_{\mathcal{H}^{m-1}}^{2} d t+\int_{0}^{T_{0}}\|\Delta p(t)\|_{\mathcal{H}^{2}}^{2} d t+\int_{0}^{T_{0}}\|\nabla v(t)\|_{\mathcal{H}^{m-1}}^{4} d t \leq \tilde{C}_{1}<\infty \\
0<\frac{1}{2 C_{0}} \leq \rho(t) \leq 2 C_{0}<\infty, \quad \forall t \in\left[0, T_{0}\right]
\end{gathered}
$$

and

$$
\lim _{\varepsilon \rightarrow 0} \sup _{t \in\left[0, T_{0}\right]}\left\|\left(\varrho^{\varepsilon, \sigma}-\varrho, v^{\varepsilon, \sigma}-v\right)(t)\right\|_{L^{\infty}}+\left|h^{\varepsilon, \sigma}-h\right|_{W^{1, \infty}}=0,
$$

where $(\varrho, v, h)$ is the localized version of $(\rho, u, F)$. Furthermore, $(\rho, u, F)$ is the unique solution to the free surface Euler equations

$$
\left\{\begin{array}{l}
\partial_{t} \rho+\operatorname{div}(\rho u)=0, \\
\rho \partial_{t} u+\rho(u \cdot \nabla) u+\nabla p=0,
\end{array} \quad x \in \Omega_{t}\right.
$$

with the boundary conditions

$$
\partial_{t} F+u \cdot \nabla F=0, \quad \text { and } p=p_{e}, \quad x \in \Sigma_{t},
$$

where $\Omega_{t}$ is the domain occupied by the fluid on time $t \geq 0$ and the boundary of $\Omega_{t}$ is given by

$$
\Sigma_{t}=\left\{x \in \mathbb{R}^{3} \mid F(x, t)=0\right\} .
$$

Remark 1.12 Based on the uniform regularity (1.47)-1.48), for any fixed $\varepsilon>0$, one can also obtain the zero surface tension limit of free surface compressible Navier-Stokes system with surface tension by the similar strong compactness argument above.

\subsection{Sketch of the proof}

Since the classical local existence results to smooth solutions of (1.1) are available [40, 63, the main difficulty in the proof of Theorem [1.5 is to get the a priori estimates of the solution in a small time 
interval independent of $\varepsilon$ and $\sigma$. For notational convenient, we drop the superscript $\varepsilon$ in the a priori estimates. Define

$$
\begin{gathered}
\Theta_{m}(p, v, h)(T)=\sup _{0 \leq t \leq T}\left(1+\|(p, v, h)(t)\|_{X_{m}^{\varepsilon}}^{2}\right)+\int_{0}^{T}\|\nabla p(t)\|_{\mathcal{H}^{m-1}}^{2}+\|\Delta p(t)\|_{\mathcal{H}^{2}}^{2}+\|\nabla v(t)\|_{\mathcal{H}^{m-1}}^{4} d t \\
+\varepsilon \int_{0}^{T}\|\nabla v(t)\|_{\mathcal{H}^{m}}^{2}+\left\|\nabla^{2} v(t)\right\|_{\mathcal{H}^{m-2}}^{2} d t+\varepsilon^{2} \int_{0}^{T}\left\|\nabla^{2} v(t)\right\|_{\mathcal{H}^{m-1}}^{2} d t<+\infty
\end{gathered}
$$

The major goal of this paper will be to derive a uniform bound of the above quantity in a finite time interval. We outline the main steps and ideas for the a priori estimates as follows:

Step 1: Conormal energy estimates for $(p, v, h)$. The first step will be to estimate $\mathcal{Z}^{\alpha}(p, v, h)$ for $|\alpha| \leq m$. The basic energy estimate $(\alpha=0)$ of $(p, v, h)$ is easy to obtain from the total energy identity of compressible Navier-Stokes system. In order to get the estimates for higher order conormal derivatives, one can perform the $L^{2}$ energy estimates on the Alinhac good unknowns $Q^{\alpha}=\mathcal{Z}^{\alpha} p-\partial_{z}^{\varphi} p \mathcal{Z}^{\alpha} \eta, V^{\alpha}=$ $\mathcal{Z}^{\alpha} v-\partial_{z}^{\varphi} v \mathcal{Z}^{\alpha} \eta$ for $|\alpha| \neq 0$ to overcome the loss of $\frac{1}{2}$ derivative for $h$ on the free surfaces as in [32], when $\sigma=0$. However, one subtle difficulty arises on the uniform estimate of boundary terms for $V^{\alpha}$ and $Q^{\alpha}$. This is because the following boundary estimate

$$
\left|\left(\partial_{z} v\right)^{b}\right|_{s} \lesssim \Lambda\left(\|v\|_{1, \infty}+|h|_{2, \infty}\right)\left(|v|_{s+1}+|h|_{s+1}\right),
$$

which plays an key role in the analysis of [32, is invalid for the compressible case due to $\operatorname{div}^{\varphi} v$ is not free. Here and henceforth, ()$^{b}$ denotes the function taking value on the boundary $z=0$. So, in general, the term $\varepsilon^{2} \int_{0}^{t}\left\|\nabla^{2} v\right\|_{\mathcal{H} \text { m-1 }}^{2} d \tau$ will appear on the right hand side of the estimate for $V^{\alpha}$ and $Q^{\alpha}$, when one applies directly the trace estimates to terms involving $\varepsilon^{2} \int_{0}^{t}\left|\left(\partial_{z} v\right)^{b}\right|_{\mathcal{H}^{m-1}}^{2} d \tau(\mathrm{cf}$. Lemma 3.3). Fortunately, with a $\varepsilon^{2}$ there, this term can be bounded by the dissipation terms in the estimates for normal derivatives and $\operatorname{div}^{\varphi} v$ in the next steps. When the effect of $\operatorname{surface} \operatorname{tension}(\sigma \neq 0)$ is taken into consideration, the boundary term involving the mean curvature $\sigma \nabla_{y} \cdot \frac{\nabla_{y} h}{\sqrt{1+\left|\nabla_{y} h\right|^{2}}}$ of the free surface seems difficult to be bounded uniformly, since

$$
\sigma \int \nabla_{y} \cdot \mathcal{Z}^{\alpha} \frac{\nabla_{y} h}{\sqrt{1+\left|\nabla_{y} h\right|^{2}}}\left(\partial_{t}^{m-1} v_{y}^{b} \cdot \partial_{t} \nabla_{y} h\right) d y \cong \sigma\left|\nabla_{y}^{2} h\right|_{\mathcal{H}^{m-1}}^{2}, \text { or } \sigma\left|v^{b}\right|_{\mathcal{H}^{m}}^{2},
$$

which both terms are difficult to be bounded uniformly (cf. Lemma 3.5). To deal with this boundary term, we will take full advantage of the third component of dynamic boundary condition (3.26) and momentum equations (3.6) to turn it into the volume integral (cf. (4.2.16)-(4.2.21)). The key observation is that the worst boundary terms appearing in (4.2.17) and 4.2.21) by this approach will be canceled with each other. It should be reminded that, for any fixed $\sigma>0$, one can derive the uniform in $\varepsilon$ and $\sigma$ estimates without using the Alinhac good unknowns since the boundary regularity can be improved due to the surface tension effect. But, when one studies the zero surface tension limit problem, this improved regularity of boundary disappear so that the Alinhac good unknowns should be used here. Precisely, one can obtain that

$$
\begin{aligned}
& \left\|\left(V^{m}, Q^{m}\right)(t)\right\|+\left|\left(h, \sqrt{\sigma} \nabla_{y} h\right)(t)\right|_{\mathcal{H}^{m}}^{2}+\varepsilon \int_{0}^{t}\left\|\nabla V^{m}\right\|^{2} d \tau \\
& \leq \Lambda\left(\tilde{C}_{0}\right)+\delta \int_{0}^{t}\|\nabla p\|_{\mathcal{H}^{m-1}}^{2}+\varepsilon^{2}\left\|\nabla^{2} v\right\|_{\mathcal{H}^{m-1}}^{2} d \tau+\Lambda\left(\Theta_{m}\right) \int_{0}^{t}\|\nabla v\|_{\mathcal{H}^{m-1}}^{2} d \tau+C_{\delta} t \Lambda\left(\Theta_{m}\right) .
\end{aligned}
$$

provided the Taylor sign condition (1.41) holds true.

Step 2: Estimates for $\left(\nabla^{\varphi} p, d_{i v}^{\varphi} v\right)$. Next, one needs to bound $\nabla p$ to close the argument. We shall use an energy method to estimate $\nabla p$, since the pressure $p=\varrho^{\gamma}$, shown in (1.31) ${ }_{2}$ for compressible flows, satisfies a transport equation due to 1.31). Thus, one also needs to bound $\operatorname{div}^{\varphi} v$. This is different from the estimate of pressure in [32, 52] for incompressible flows, which satisfies an elliptic equation due to the divergence free condition. Furthermore, since

$$
\partial_{z} v \cong \operatorname{div}^{\varphi} v+\Pi\left(\partial_{z} v\right)+\nabla_{y} v
$$


the estimate of $\operatorname{div}^{\varphi} v$ is also helpful to the estimates of normal derivative $\partial_{z} v$. It follows from the standard energy method that

$$
\begin{aligned}
& \|\nabla v(t)\|_{\mathcal{H}^{m-2}}^{2}+\varepsilon\left\|\left(\operatorname{div}^{\varphi} v, \nabla^{\varphi} p\right)(t)\right\|_{\mathcal{H}^{m-1}}^{2}+\int_{0}^{t} \varepsilon\left\|\nabla^{\varphi} \mathcal{Z}^{m-2} \operatorname{div}^{\varphi} v\right\|^{2}+\varepsilon^{2}\left\|\nabla^{\varphi} \mathcal{Z}^{m-1} \operatorname{div}^{\varphi} v\right\|^{2} d \tau \\
& \leq \Lambda\left(\tilde{C}_{0}\right)+\delta \varepsilon^{2} \int_{0}^{t}\left\|\nabla^{2} v\right\|_{\mathcal{H}^{m-1}}^{2} d \tau+\Lambda\left(\Theta_{m}\right) \int_{0}^{t}\|\nabla v\|_{\mathcal{H}^{m-1}}^{2} d \tau+C_{\delta} t \Lambda\left(\Theta_{m}\right) \\
& \|\nabla p(t)\|_{\mathcal{H}^{m-2}}^{2}+\int_{0}^{t}\|\nabla p\|_{\mathcal{H}^{m-1}}^{2} d \tau \leq \Lambda\left(\tilde{C}_{0}\right)+\varepsilon^{2} \int_{0}^{t}\left\|\nabla^{2} v\right\|_{\mathcal{H}^{m-1}}^{2} d \tau+t \Lambda\left(\Theta_{m}\right) \\
& \left\|\operatorname{div}^{\varphi} v\right\|_{\mathcal{H}^{m-1}}^{2} \leq \Lambda\left(\Theta_{m}\right)
\end{aligned}
$$

Here the estimate of $\|(\nabla v, \nabla p)(t)\|_{\mathcal{H}^{m-2}}^{2}$ is a consequence of the fundamental theorem of calculus. It should be reminded that the estimate of

$$
\varepsilon\left\|\operatorname{div}^{\varphi} v(t)\right\|_{\mathcal{H}^{m-1}}^{2}+\int_{0}^{t} \varepsilon\left\|\nabla^{\varphi} \mathcal{Z}^{m-2} \operatorname{div}^{\varphi} v\right\|^{2}+\varepsilon^{2}\left\|\nabla^{\varphi} \mathcal{Z}^{m-1} \operatorname{div}^{\varphi} v\right\|^{2} d \tau
$$

is the key to bound $\varepsilon\|\nabla v(t)\|_{\mathcal{H}^{m-1}}^{2}+\int_{0}^{t} \varepsilon\left\|\nabla^{2} v\right\|_{\mathcal{H}^{m-2}}^{2}+\varepsilon^{2}\left\|\nabla^{2} v\right\|_{\mathcal{H}^{m-1}}^{2} d \tau$. This is not necessary for incompressible case, since $\int_{0}^{t} \varepsilon\left\|\nabla^{2} v\right\|_{\mathcal{H}^{m-2}}^{2}+\varepsilon^{2}\left\|\nabla^{2} v\right\|_{\mathcal{H}^{m-1}}^{2} d \tau$ does not appear due to (1.62).

Step 3: Normal derivative estimates Part I. Since $\nabla_{y} v$ and $\operatorname{div}^{\varphi} v$ have been bounded in the above steps, in order to bound $\partial_{z} v$, it remains to estimate $\Pi\left(\partial_{z} v\right)$, where $\Pi=I d-\mathbf{n} \otimes \mathbf{n}$ denotes the tangential vector field. One can follow the arguments in 32 to derive the estimate for

$$
S_{\mathbf{n}}=\Pi\left(S^{\varphi} v \mathbf{N}\right)
$$

which is equivalent to $\Pi\left(\partial_{z} v\right)$ but vanishes on the boundary. The main conclusion of this step is

$$
\begin{aligned}
& \left\|S_{\mathbf{n}}(t)\right\|_{\mathcal{H}^{m-2}}^{2}+\varepsilon\left\|S_{\mathbf{n}}(t)\right\|_{\mathcal{H}^{m-1}}^{2}+\int_{0}^{t} \varepsilon\left\|\nabla^{\varphi} \mathcal{Z}^{m-2} S_{\mathbf{n}}\right\|^{2}+\varepsilon^{2}\left\|\nabla^{\varphi} \mathcal{Z}^{m-1} S_{\mathbf{n}}\right\|^{2} d \tau \\
& \leq \Lambda\left(\tilde{C}_{0}\right)+\Lambda\left(\Theta_{m}\right) \int_{0}^{t}\|\nabla v\|_{\mathcal{H}^{m-1}}^{2} d \tau+C_{\delta} t \Lambda\left(\Theta_{m}\right) .
\end{aligned}
$$

This, together with (1.64), yields immediately that $\varepsilon\|\nabla v(t)\|_{\mathcal{H}^{m-1}}^{2}+\int_{0}^{t} \varepsilon\left\|\nabla^{2} v\right\|_{\mathcal{H}^{m-2}}^{2}+\varepsilon^{2}\left\|\nabla^{2} v\right\|_{\mathcal{H}^{m-1}}^{2} d \tau$, which does not appear in the arguments of 32 due to the divergence free condition. It should be noted that $\varepsilon\|(\nabla v, \nabla p)(t)\|_{\mathcal{H}^{m-1}}^{2}$ is necessary to close the estimate of $\int_{0}^{t} \varepsilon^{2}\left\|\nabla^{2} v\right\|_{\mathcal{H}^{m-1}}^{2} d \tau$.

Step 4: Normal derivative estimates Part II. In order to close the a priori estimate, it remains to bound the $m-1$ order conormal derivatives of $\partial_{z} v$. As indicated in 32, one can only expect the estimate of $\int_{0}^{t}\|\nabla v\|_{\mathcal{H}^{m-1}}^{4} d \tau$. Based on the estimate of $\operatorname{div}^{\varphi} v$ in Step 2 and the fact $\partial_{z} v \cong \operatorname{div}^{\varphi} v+\omega \times \mathbf{N}+\nabla_{y} v$, it suffices to bound $\int_{0}^{t}\|\omega \times \mathbf{N}\|_{\mathcal{H}^{m-1}}^{4} d \tau$. Due to the less regularity of $\nabla p$ and $\operatorname{div}^{\varphi} v$, one chooses to bound $\omega \times \mathbf{N}$ as in 32 instead of $S_{\mathbf{n}}$, since $\nabla^{\varphi} p$ and $\nabla^{\varphi} \operatorname{div}^{\varphi} v$ can be eliminated in the equation of the vorticity $\omega$. We will follow the approach of microlocal symmetrizer in 32 to derive this kind of estimate. However, if one applies directly this argument to $\int_{0}^{t}\|\omega\|_{\mathcal{H}^{m-1}}^{4} d \tau$ as 32 so that

$$
\left(\int_{0}^{t}\|\omega\|_{\mathcal{H}^{m-1}}^{4} d \tau\right)^{\frac{1}{2}} \lesssim \Lambda\left(\tilde{C}_{0}\right)+\sqrt{\varepsilon} \int_{0}^{t}\left|\omega^{b}(\tau)\right|_{\mathcal{H}^{m-1}}^{2} d \tau+t^{\frac{1}{2}} \Lambda\left(\Theta_{m}\right) .
$$

Then it seems difficult to bound $\sqrt{\varepsilon} \int_{0}^{t}\left|\omega^{b}(\tau)\right|_{\mathcal{H}^{m-1}}^{2} d \tau$, since it involves $\left|\left(\partial_{z} v\right)^{b}\right|_{\mathcal{H}^{m-1}}$ but in general (1.62) is not valid for the compressible flows. It should be noted that even though it follows from trace theorem that

$$
\sqrt{\varepsilon} \int_{0}^{t}|\omega(\tau)|_{\mathcal{H}^{m-1}}^{2} d \tau \lesssim \sqrt{\varepsilon} \int_{0}^{t}\|\nabla \omega\|_{\mathcal{H}^{m-1}}\|\omega\|_{\mathcal{H}^{m-1}} d \tau+t^{\frac{1}{2}} \Lambda\left(\Theta_{m}\right) \lesssim \varepsilon \int_{0}^{t}\|\nabla \omega\|_{\mathcal{H}^{m-1}}^{2} d \tau+t^{\frac{1}{2}} \Lambda\left(\Theta_{m}\right),
$$

but, $\varepsilon \int_{0}^{t}\|\nabla \omega\|_{\mathcal{H}^{m-1}}^{2} d \tau$ may not be expected to be bounded. However, direct calculations yield(c.f. (4.4.5) and (4.5.2) that

$$
(\omega \times \mathbf{N})^{b}=-2 \Pi\left\{\left(\partial_{1} v \cdot \mathbf{N}, \partial_{2} v \cdot \mathbf{N}, 0\right)^{t}\right\}
$$


So, one can expect to bound $\int_{0}^{t}\|\omega \times \mathbf{N}\|_{\mathcal{H}^{m-1}}^{4} d \tau$. To avoid involving too much regularity of $h$ (due to the commutator for $\mathcal{Z}^{m-1} \Delta^{\varphi} \omega \times \mathbf{N}$ ), it is equivalent to bound $\int_{0}^{t}\left\|\mathcal{Z}^{m-1} \omega \times \mathbf{N}\right\|^{4} d \tau$ base on the fact

$$
\int_{0}^{t}\|\omega \times \mathbf{N}\|_{\mathcal{H}^{m-1}}^{4} d \tau \lesssim \int_{0}^{t}\left\|\mathcal{Z}^{m-1} \omega \times \mathbf{N}\right\|^{4} d \tau+t \Lambda\left(\Theta_{m}\right) .
$$

Note that the boundary condition $(1.33)$ yields

$$
\left(\mathcal{Z}^{m-1} \omega \times \mathbf{N}\right)^{b} \cong \mathcal{Z}^{m-1} \nabla_{y} h+\left(\mathcal{Z}^{m-1} \nabla_{y} v\right)^{b}+\left(\mathcal{Z}^{m-2} \omega\right)^{b}+\text { l.o.t. }
$$

Then, it follows from the argument of the microlocal symmetrizer in 32 that

$$
\begin{aligned}
\int_{0}^{t}\|\omega \times \mathbf{N}\|_{\mathcal{H}^{m-1}}^{4} d \tau & \lesssim \Lambda\left(\tilde{C}_{0}\right)+\sqrt{\varepsilon} \Lambda\left(\Theta_{m}\right) \int_{0}^{t}\left|\left(\mathcal{Z}^{m-1} \omega \times \mathbf{N}\right)\right|_{L^{2}}^{2} d \tau+t \Lambda\left(\Theta_{m}\right) \\
& \lesssim \Lambda\left(\tilde{C}_{0}\right)+\varepsilon \int_{0}^{t}\left\|\nabla V^{m}\right\|^{2}+\left\|\nabla^{2} v\right\|_{\mathcal{H}^{m-2}}^{2} d \tau+t \Lambda\left(\Theta_{m}\right) .
\end{aligned}
$$

Therefore, the conclusion of this step is

$$
\left(\int_{0}^{t}\|\nabla v\|_{\mathcal{H}^{m-1}}^{4}\right)^{\frac{1}{2}} \lesssim \Lambda\left(\tilde{C}_{0}\right)+\varepsilon \int_{0}^{t}\left\|\nabla V^{m}\right\|^{2}+\left\|\nabla^{2} v\right\|_{\mathcal{H}^{m-2}}^{2} d \tau+t \Lambda\left(\Theta_{m}\right) .
$$

Step 5: $L^{\infty}$-estimates. Finally, we will bound $L^{\infty}$-norm of $\|(\nabla p, \nabla v)\|_{\mathcal{H}^{1, \infty}}$ and $\sqrt{\varepsilon}\left\|\nabla^{2} v\right\|_{L^{\infty}}$ to close the priori estimate. In order to estimate $\|\nabla p\|_{\mathcal{H}^{1, \infty}}$, using the anisotropic Sobolev inequality, one only needs to estimate $\left\|\Delta^{\varphi} p\right\|_{\mathcal{H}^{1}}$. Actually, one can obtain, for $m \geq 6$, that

$$
\left(\left\|\Delta^{\varphi} p(t)\right\|_{\mathcal{H}^{1}}^{2}+\varepsilon\left\|\Delta^{\varphi} p(t)\right\|_{\mathcal{H}^{2}}^{2}\right)+\int_{0}^{t}\left\|\mathcal{Z}^{\alpha} \Delta^{\varphi} p\right\|^{2} d \tau \lesssim \Lambda\left(\tilde{C}_{0}\right)+t \Lambda\left(\Theta_{m}\right) .
$$

This estimate also implies that the boundary layer for density is weaker than the one for velocity.

In order to estimate $\|\nabla v\|_{\mathcal{H}^{1, \infty}}$ and $\sqrt{\varepsilon}\left\|\nabla^{2} v\right\|_{L^{\infty}}$, it suffices to to estimate $\left\|S_{\mathbf{n}}\right\|_{\mathcal{H}^{1, \infty}}^{2}$ and $\varepsilon\left\|\nabla S_{\mathbf{n}}\right\|_{L^{\infty}}^{2}$, since

$$
\|\nabla v\|_{\mathcal{H}^{1, \infty}}^{2}+\varepsilon\left\|\nabla^{2} v\right\|_{L^{\infty}}^{2} \lesssim \Lambda\left(\tilde{C}_{0}\right)+\left\|\Delta^{\varphi} p\right\|_{\mathcal{H}^{1}}^{2}+\left\|S_{\mathbf{n}}\right\|_{\mathcal{H}^{1, \infty}}^{2}+\varepsilon\left\|\nabla S_{\mathbf{n}}\right\|_{L^{\infty}}^{2} .
$$

However, $\left\|S_{\mathbf{n}}\right\|_{\mathcal{H}^{1, \infty}}^{2}$ and $\varepsilon\left\|\nabla S_{\mathbf{n}}\right\|_{L^{\infty}}^{2}$ seems difficult to be bounded if one performs $L^{\infty}$ estimates directly on the convection diffusion equation solved by $S_{\mathbf{n}}$ due to the appearance of the term $\left\|\nabla \operatorname{div}^{\varphi} v\right\|_{\mathcal{H}^{2, \infty}}^{2}$, which is difficult to controll. Therefore, we try to estimate

$$
\zeta_{\mathbf{n}}=\Pi(\omega \times \mathbf{N})-2 \Pi\left\{\left(\nabla_{y}, 0\right)^{t} \partial_{t} \eta-\left(\nabla^{\varphi} \mathbf{N}\right)^{t} v\right\},
$$

which also solves a convection diffusion equation but involving $\left\|\nabla \operatorname{div}^{\varphi} v\right\|_{\mathcal{H}^{1, \infty}}$ only. By using the similar argument in 32, one can obtain, for $m \geq 6$, that

$$
\|\nabla v\|_{\mathcal{H}^{1, \infty}}^{2}+\varepsilon\left\|\nabla^{2} v\right\|_{L^{\infty}}^{2} \lesssim \Lambda\left(\tilde{C}_{0}\right)+t^{\frac{1}{2}} \Lambda\left(\Theta_{m}\right) .
$$

The estimate of $\Theta_{m}$ on some uniform time follows by collecting the estimates of the five steps. Note that at the end, we need to check that the Taylor sign condition and $\Phi$ being a differmorphism remain true.

The rest of the paper is organized as follows: In the next section, we collect some elementary facts and inequalities to be used later. In section 3 , we study the equations satisfied by $\left(\mathcal{Z}^{\alpha} p, \mathcal{Z}^{\alpha} v, \mathcal{Z}^{\alpha} h\right)$ and prove the estimates for the lower order commutators and boundary values. In section 4 , the a priori estimates Theorem 4.1 will be established, which is the main part of this paper. Using the a priori estimates, Theorem 1.5 and Theorems 1.10, 1.11 are proved in section 5. The Appendix collects a generalization of Lemma 14 and Lemma 15 for $L^{\infty}$ estimates in [31] so that it can be applied to compressible Navier-Stokes equations. 


\section{Preliminaries}

In this section, we will list some basic properties of conormal function spaces and some elementary facts which will be used frequently in the process of a priori estimates. For later use, define

$$
\begin{gathered}
\mathcal{W}^{m}([0, T] \times \mathcal{S})=\left\{f(t, y, z) \in L^{2}([0, T] \times \mathcal{S})\left|\mathcal{Z}^{\alpha} f \in L^{2}([0, T] \times \mathcal{S}), \quad\right| \alpha \mid \leq m\right\}, \\
\mathcal{W}^{m, \infty}([0, T] \times \mathcal{S})=\left\{f(t, y, z) \in L^{\infty}([0, T] \times \mathcal{S})\left|\mathcal{Z}^{\alpha} f \in L^{\infty}([0, T] \times \mathcal{S}),\right| \alpha \mid \leq m\right\},
\end{gathered}
$$

and the following notations

$$
\|f\|_{\mathcal{W}}^{2} \mathcal{W}_{t}^{m, \infty}=\sup _{0 \leq \tau \leq t} \sum_{|\alpha| \leq m}\left\|\mathcal{Z}^{\alpha} f(\tau)\right\|_{L^{\infty}(\mathcal{S})}^{2}, m \geq 1,\|f\|_{\infty, t}=\sup _{0 \leq \tau \leq t}\|f(\tau)\|_{L^{\infty}(\mathcal{S})} .
$$

\subsection{General Inequalities}

The following Gagliardo-Nirenberg-Morser type inequality will be used repeatedly whose proof can be found in [19].

Lemma 2.1 The following products and commutator estimates hold:

(1) For $u, v \in L^{\infty}([0, T] \times \mathcal{S}) \cap \mathcal{W}^{k}([0, T] \times \mathcal{S})$ with $k \in \mathbb{N}$ be an integer. It holds for any $0 \leq t \leq T$ that

$$
\int_{0}^{t}\left\|\left(\mathcal{Z}^{\beta} u \mathcal{Z}^{\nu} v\right)(\tau)\right\|^{2} d \tau \lesssim\|u\|_{\infty, t}^{2} \int_{0}^{t}\|v(\tau)\|_{\mathcal{H}^{k}}^{2} d \tau+\|v\|_{\infty, t}^{2} \int_{0}^{t}\|u(\tau)\|_{\mathcal{H}^{k}}^{2} d \tau, \quad|\beta|+|\nu|=k
$$

(2) For $1 \leq|\alpha| \leq k, 0 \leq t \leq T, g \in \mathcal{W}^{k-1}([0, T] \times \mathcal{S}) \cap L^{\infty}([0, T] \times \mathcal{S}), f \in \mathcal{W}^{k}([0, T] \times \mathcal{S})$ such that $\mathcal{Z} f \in L^{\infty}([0, T] \times \mathcal{S})$, we have

$$
\int_{0}^{t}\left\|\left[\mathcal{Z}^{\alpha}, f\right] g\right\|^{2} d \tau \lesssim\|g\|_{\infty, t}^{2} \int_{0}^{t}\|\mathcal{Z} f\|_{\mathcal{H}^{k-1}}^{2} d \tau+\|\mathcal{Z} f\|_{\infty, t}^{2} \int_{0}^{t}\|g\|_{\mathcal{H}^{k-1}}^{2} d \tau
$$

(3) For $|\alpha|=k \geq 2,0 \leq t \leq T$, and $f, g \in \mathcal{W}^{k-1}([0, T] \times \mathcal{S}) \cap \mathcal{W}^{1, \infty}([0, T] \times \mathcal{S})$. Define the symmetric commutator as $[\mathcal{Z}, f, g]=\mathcal{Z}^{\alpha}(f g)-\mathcal{Z}^{\alpha} f g-f \mathcal{Z}^{\alpha} g$. Then, it holds for any $0<t<T$ that

$$
\int_{0}^{t}\left\|\left[\mathcal{Z}^{\alpha}, f, g\right]\right\|^{2} d \tau \lesssim\|\mathcal{Z} f\|_{\infty, t}^{2} \int_{0}^{t}\|\mathcal{Z} g\|_{\mathcal{H}^{k-2}}^{2} d \tau+\|\mathcal{Z} g\|_{\infty, t}^{2} \int_{0}^{t}\|\mathcal{Z} f\|_{\mathcal{H}^{k-2}}^{2} d \tau
$$

We also need the following anisotropic Sobolev embedding and trace estimates in $\mathcal{S}$ whose proof can be found in 32 :

Lemma 2.2 Let $m_{1} \geq 0, m_{2} \geq 0$ be integers, $f \in H_{c o}^{m_{1}}(\mathcal{S}) \cap H_{c o}^{m_{2}}(\mathcal{S})$ and $\nabla f \in H_{c o}^{m_{2}}(\mathcal{S})$.

1) The following the anisotropic Sobolev embedding holds:

$$
\|f\|_{L^{\infty}}^{2} \leq C\left(\|\nabla f\|_{m_{2}}+\|f\|_{m_{2}}\right)\|f\|_{m_{1}} .
$$

provided $m_{1}+m_{2} \geq 3$.

2) The following trace estimate holds:

$$
|f|_{H^{s}\left(\mathbb{R}^{2}\right)}^{2} \leq C\left(\|\nabla f\|_{m_{2}}+\|f\|_{m_{2}}\right)\|f\|_{m_{1}} .
$$

with $m_{1}+m_{2} \geq 2 s \geq 0$.

The following classical Sobolev inequalities and commutator estimates in $\mathbb{R}^{2}$ hold:

Lemma 2.3 For $s \in \mathbb{R}, s \geq 0$, it holds that

$$
\begin{aligned}
& |f g|_{H^{s}\left(\mathbb{R}^{2}\right)} \leq C_{s}\left(|f|_{L^{\infty}\left(\mathbb{R}^{2}\right)}|g|_{H^{s}\left(\mathbb{R}^{2}\right)}+|g|_{L^{\infty}\left(\mathbb{R}^{2}\right)}|f|_{H^{s}\left(\mathbb{R}^{2}\right)}\right) \\
& \left|\left[\mathcal{F}^{s}, f\right] \nabla g\right| \leq C_{s}\left(|\nabla f|_{L^{\infty}\left(\mathbb{R}^{2}\right)}|g|_{H^{s}\left(\mathbb{R}^{2}\right)}+|\nabla g|_{L^{\infty}\left(\mathbb{R}^{2}\right)}|f|_{H^{s}\left(\mathbb{R}^{2}\right)}\right), \\
& |u v|_{\frac{1}{2}} \lesssim|u|_{1, \infty}|v|_{\frac{1}{2}}
\end{aligned}
$$

where $\mathcal{F}^{s}$ is a Fourier multiplier of $\left(1+|\xi|^{2}\right)^{\frac{s}{2}}$. 


\subsection{Estimates involving $\varphi$}

We shall derive some estimates of $\eta$ chosen by (1.19) and (1.20) in terms of $h$ and $v$. It shows that $\eta$ has $\frac{1}{2}$ higher order standard Sobolev regularity in $\mathcal{S}$ than $h$. Note that the different choice of cut-off function $\kappa$ in (1.20) from the one in [32] allows one to control the $L^{2}$ norm of $\eta$ itself. Assume that $A$ is chosen such that $\partial_{z} \varphi_{0}(y, z) \geq 1$ at the initial time and

$$
\partial_{z} \varphi(t, y, z) \geq c_{0}, \quad \forall t \in\left[0, T^{\varepsilon}\right]
$$

for some $c_{0}>0$ and small time $T^{\varepsilon}$. With this a priori assumption, one obtains that

Lemma 2.4 It holds for $\eta$, defined in (1.19), (1.20), that:

$$
\begin{aligned}
& \forall k \in \mathbb{N}, \quad\|\eta\|_{H^{k}(\mathcal{S})} \leq C_{k}|h|_{k-\frac{1}{2}}, \\
& \forall k, l \in \mathbb{N}, \quad\left\|\partial_{t}^{l} \eta\right\|_{H^{k}(\mathcal{S})} \leq C_{k}\left|\partial_{t}^{l} h\right|_{k-\frac{1}{2}},
\end{aligned}
$$

and moreover, we also have the $L^{\infty}$ estimates

$$
\begin{aligned}
& \forall k \in \mathbb{N}, \quad\|\eta\|_{W^{k, \infty}} \leq C_{k}|h|_{k, \infty}, \\
& \forall k, l \in \mathbb{N}, \quad\left\|\partial_{t}^{l} \eta\right\|_{W^{k, \infty}} \leq C_{k}\left|\partial_{t}^{l} h\right|_{k, \infty} .
\end{aligned}
$$

Proof. It follows from the expressions (1.19) and (1.20) that

$$
\begin{aligned}
\|\eta\|^{2} & =\int_{-\infty}^{0} d z \int_{\mathbb{R}^{2}}|\hat{\eta}(t, \xi, z)|^{2} d \xi \leq \int_{\mathbb{R}^{2}}|\hat{h}(t, \xi)|^{2} d \xi \int_{-\infty}^{0}|\kappa(z\langle\xi\rangle)|^{2} d z \\
& \lesssim \int_{\mathbb{R}^{2}}\langle\xi\rangle^{-1}|\hat{h}(t, \xi)|^{2} d \xi=|h|_{-\frac{1}{2}} .
\end{aligned}
$$

The proof of remaining parts is similar to [31, so we omit the details here.

Lemma 2.1 and 2.4 yield immediately the following estimates which will be used repeatly, whose proof can be found in 32 .

Lemma 2.5 For any $m \in \mathbb{N}$, it holds that

$$
\int_{0}^{t}\left\|\frac{f}{\partial_{z} \varphi}\right\|_{\mathcal{H}^{m}}^{2} d \tau \lesssim \Lambda\left(\frac{1}{c_{0}},\|f\|_{\infty, t}+|h|_{\mathcal{W}_{t}^{1, \infty}}\right) \int_{0}^{t}\|f\|_{\mathcal{H}^{m}}^{2}+\left|\mathcal{Z}^{m} h\right|_{\frac{1}{2}}^{2} d \tau
$$

and moreover, for standard Sobolev norms, we also have that, for $0 \leq k \leq m$,

$$
\int_{0}^{t}\left\|\partial_{t}^{k}\left(\frac{f}{\partial_{z} \varphi}\right)\right\|_{H^{m-k}}^{2} d \tau \leq \Lambda\left(\frac{1}{c_{0}},\|f\|_{\infty, t}+|h|_{\mathcal{W}_{t}^{1, \infty}}\right) \int_{0}^{t}\left(\sum_{k=0}^{m}\left\|\partial_{t}^{k} f\right\|_{H^{m-k}}^{2}+\left|\partial_{t}^{k} h\right|_{m-k+\frac{1}{2}}^{2}\right) d \tau .
$$

As a consequence of Lemma 2.5, one has

Corollary 2.6 For any $m \geq 1$ and any sufficiently smooth function $f$, it holds that

$$
\begin{aligned}
\int_{0}^{t}\left\|\nabla^{\varphi} f\right\|_{\mathcal{H}^{m}}^{2} d \tau \leq & \Lambda\left(\frac{1}{c_{0}},|h|_{\mathcal{W}_{t}^{1, \infty}}\right) \int_{0}^{t}\|\nabla f\|_{\mathcal{H}^{m}}^{2} d \tau \\
& +\Lambda\left(\frac{1}{c_{0}},\left\|\partial_{z} f\right\|_{\infty, t}+|h|_{\mathcal{W}_{t}^{2, \infty}}\right) \int_{0}^{t}\left\|\partial_{z} f\right\|_{\mathcal{H}^{m-1}}^{2}+\left|\mathcal{Z}^{m} h\right|_{\frac{1}{2}}^{2} d \tau \\
\int_{0}^{t}\|\nabla f\|_{\mathcal{H}^{m}}^{2} d \tau \leq & \Lambda\left(\frac{1}{c_{0}},|h|_{\mathcal{W}_{t}^{1, \infty}}\right) \int_{0}^{t}\left\|\nabla^{\varphi} f\right\|_{\mathcal{H}^{m}}^{2} d \tau \\
& +\Lambda\left(\frac{1}{c_{0}},\left\|\partial_{z} f\right\|_{\infty, t}+|h|_{\mathcal{W}_{t}^{2, \infty}}\right) \int_{0}^{t}\left\|\partial_{z} f\right\|_{\mathcal{H}^{m-1}}^{2}+\left|\mathcal{Z}^{m} h\right|_{\frac{1}{2}}^{2} d \tau
\end{aligned}
$$

and

$$
\begin{aligned}
\int_{0}^{t}\left\|\nabla^{\varphi} \nabla^{\varphi} f\right\|_{\mathcal{H}^{m}}^{2} d \tau \leq & \Lambda\left(\frac{1}{c_{0}},|h|_{\mathcal{W}_{t}^{2, \infty}}\right)\left\{\int_{0}^{t}\left\|\nabla^{2} f\right\|_{\mathcal{H}^{m}}^{2} d \tau+\int_{0}^{t}\|\nabla f\|_{\mathcal{H}^{m}}^{2} d \tau\right. \\
& \left.+\left\|\nabla^{2} f\right\|_{\infty, t}^{2} \int_{0}^{t}\left|\mathcal{Z}^{m} h\right|_{\frac{1}{2}}^{2} d \tau+\|\nabla f\|_{\infty, t}^{2} \int_{0}^{t}\left|\nabla_{y} \mathcal{Z}^{m} h\right|_{\frac{1}{2}}^{2} d \tau\right\} .
\end{aligned}
$$


Proof. These estimates follow from (2.2), (2.8) and the identity, for $i=1,2,3$,

$$
\mathcal{Z}^{\alpha}\left(\partial_{i}^{\varphi} f\right)=\mathcal{Z}^{\alpha} \partial_{i} f-\frac{\partial_{i} \varphi}{\partial_{z} \varphi} \mathcal{Z}^{\alpha} \partial_{z} f-\left[\mathcal{Z}^{\alpha}, \frac{\partial_{i} \varphi}{\partial_{z} \varphi}\right] \partial_{z} f
$$

where $\partial_{i} \varphi$ should be replaced by -1 when $i=3$.

Corollary 2.7 For $1 \leq|\alpha| \leq k, 0<t<T$, and any sufficiently smooth function $f$, it holds that

$$
\int_{0}^{t}\left\|\left[\partial_{i}^{\varphi}, \mathcal{Z}^{\alpha}\right] f\right\|^{2} d \tau \leq \Lambda\left(\frac{1}{c_{0}},\|\nabla f\|_{\infty, t}+|h|_{\mathcal{W}_{t}^{2, \infty}}\right) \int_{0}^{t}\|\nabla f\|_{\mathcal{H}^{k-1}}^{2}+\left|\mathcal{Z}^{k} h\right|_{\frac{1}{2}}^{2} d \tau
$$

Proof. This corollary follows from (2.2), (2.8) and the identity, for $i=1,2,3$,

$$
\left[\partial_{i}^{\varphi}, \mathcal{Z}^{\alpha}\right] f=\left[\mathcal{Z}^{\alpha}, \frac{\partial_{i} \varphi}{\partial_{z} \varphi}\right]\left(\partial_{z} f\right)+\frac{\partial_{i}^{\varphi}}{\partial_{z} \varphi}\left[\mathcal{Z}^{\alpha}, \partial_{z}\right]
$$

where $\partial_{i} \varphi$ should be replaced by -1 when $i=3$.

The following lemma gives the regularity of free surfaces once we gain the corresponding one of the velocity.

Lemma 2.8 For any $m \in \mathbb{N}, \varepsilon \in(0,1]$, it holds that

$$
\varepsilon\left|\mathcal{Z}^{m} h\right|_{\frac{1}{2}}^{2} d \tau \leq \varepsilon\left|\mathcal{Z}^{m} h_{0}\right|_{\frac{1}{2}}^{2}+\varepsilon \int_{0}^{t}\|\nabla v\|_{\mathcal{H}^{m}}^{2} d \tau+\Lambda\left(\left.|\nabla|_{y} h\right|_{\infty, t}+\|v\|_{\mathcal{W}_{t}^{1, \infty}}\right) \int_{0}^{t}\left(\|v\|_{\mathcal{H}^{m}}^{2}+\varepsilon\left|\mathcal{Z}^{m} h\right|_{\frac{1}{2}}^{2}\right) d \tau \text {. }
$$

Proof. The proof of this lemma is similar to Proposition 3.4 in [32] except for the time derivative involving. By taking time derivative in the boundary condition (1.32) and following the same argument in [32, we can prove this lemma. The details are omitted here for brevity.

Since the Jacobian of the change of variable (1.24) is $\partial_{z} \varphi$, it is natural to use on $\mathcal{S}$ the following weighted $L^{2}$ scalar products:

$$
\int_{\mathcal{S}} f g d \mathcal{V}_{t}, \text { with } d \mathcal{V}_{t}=\partial_{z} \varphi(t, y, z) d y d z
$$

With this notation, one has the following integration by parts identities for the operators $\partial_{i}^{\varphi}$ :

Lemma 2.9 For any sufficient smooth function $f$ and $g$, it holds that

$$
\begin{aligned}
& \int_{\mathcal{S}} \partial_{i}^{\varphi} f g d \mathcal{V}_{t}=-\int_{\mathcal{S}} f \partial_{i}^{\varphi} g d \mathcal{V}_{t}+\int_{z=0} f g \mathbf{N}_{i} d y, \quad i=1,2,3, \\
& \int_{\mathcal{S}} \partial_{t}^{\varphi} f g d \mathcal{V}_{t}=\partial_{t} \int_{\mathcal{S}} f g d \mathcal{V}_{t}-\int_{\mathcal{S}} f \partial_{t}^{\varphi} g d \mathcal{V}_{t}-\int_{z=0} f g \partial_{t} h d y,
\end{aligned}
$$

where $\mathbf{N}=\left(-\partial_{1} h,-\partial_{2} h, 1\right)^{t}$.

The following Korn's inequality is also needed(see [32] for the proof).

Lemma 2.10 Assume that $\partial_{z} \varphi \geq c_{0}$ and $\|\nabla \varphi\|_{L^{\infty}}+\left\|\nabla^{2} \varphi\right\|_{L^{\infty}} \leq \frac{1}{c_{0}}$ for some $c_{0}>0$, then there exists $\Lambda_{0}:=\Lambda\left(\frac{1}{c_{0}}\right)$, such that for every $v \in H^{1}(\mathcal{S})$, it holds that

$$
\|\nabla v\|_{L^{2}(\mathcal{S})}^{2} \leq \Lambda_{0} \int_{\mathcal{S}}\left|\nabla^{\varphi} v\right|^{2} d \mathcal{V}_{t}
$$

and

$$
\|\nabla v\|_{L^{2}(\mathcal{S})}^{2} \leq \Lambda_{0}\left(\int_{\mathcal{S}}\left|S^{\varphi} v\right|^{2} d \mathcal{V}_{t}+\|v\|_{L^{2}(\mathcal{S})}^{2}\right),
$$

where $S^{\varphi} v=\frac{1}{2}\left(\nabla^{\varphi} v+\left(\nabla^{\varphi} v\right)^{t}\right)$. 


\section{Equations for higher order conormal derivative terms}

In this section, we derive the equations for $\left(\mathcal{Z}^{\alpha} v, \mathcal{Z}^{\alpha} p, \mathcal{Z}^{\alpha} \eta\right)$ involving high order conormal derivatives. As explained in [32, the commutators between $\mathcal{Z}^{\alpha}$ and $\partial_{t}^{\varphi}+v \cdot \nabla^{\varphi}$ or $\nabla^{\varphi} p$ in the equations yields a loss of $\frac{1}{2}$ derivative due to the lower regularity of $\varphi$ when $\sigma$ is not a given constant independent of $\varepsilon$. We shall introduce the Alinhac good unknowns 1] to yields some nonlinear cancellation in the equations so that the commutators are all the lower order terms.

\subsection{A commutator estimate}

In order to perform higher order conormal estimates, we first derive the equations satisfied by $\left(\mathcal{Z}^{\alpha} v, \mathcal{Z}^{\alpha} p\right)$. We thus need to commute the conoraml vector field $\mathcal{Z}^{\alpha}$ with each term in the equation (1.31). The following commutation relations are needed, which has been shown in 32 except for $Z_{0}=\partial_{t}$.

It is easy to check that for $i=0,1,2,3$, and a smooth function $f$, one has

$$
\mathcal{Z}^{\alpha} \partial_{i}^{\varphi} f=\partial_{i}^{\varphi} \mathcal{Z}^{\alpha} f-\partial_{z}^{\varphi} f \partial_{i}^{\varphi} \mathcal{Z}^{\alpha} \eta+\mathcal{C}_{i}^{\alpha}(f)
$$

where the commutator $\mathcal{C}_{i}^{\alpha}(f)$ is given for $\alpha \neq 0$ and $i \neq 3$ by

$$
\mathcal{C}_{i}^{\alpha}(f)=\mathcal{C}_{i, 1}^{\alpha}(f)+\mathcal{C}_{i, 2}^{\alpha}(f)+\mathcal{C}_{i, 3}^{\alpha}(f),
$$

with

$$
\begin{aligned}
& \mathcal{C}_{i, 1}^{\alpha}(f)=-\left[\mathcal{Z}^{\alpha}, \frac{\partial_{i} \varphi}{\partial_{z} \varphi}, \partial_{z} f\right] \\
& \mathcal{C}_{i, 2}^{\alpha}(f)=-\partial_{z} f\left[\mathcal{Z}^{\alpha}, \partial_{i} \varphi, \frac{1}{\partial_{z} \varphi}\right]-\partial_{i} \varphi\left(\mathcal{Z}^{\alpha}\left(\frac{1}{\partial_{z} \varphi}\right)+\frac{\mathcal{Z}^{\alpha} \partial_{z} \eta}{\left(\partial_{z} \varphi\right)^{2}}\right) \partial_{z} f, \\
& \mathcal{C}_{i, 3}^{\alpha}(f)=-\frac{\partial_{i} \varphi}{\partial_{z} \varphi}\left[\mathcal{Z}^{\alpha}, \partial_{z}\right] f+\frac{\partial_{i} \varphi}{\left(\partial_{z} \varphi\right)^{2}} \partial_{z} f\left[\mathcal{Z}^{\alpha}, \partial_{z}\right] \eta
\end{aligned}
$$

For $i=3$, we only need to replace $\partial_{i} \varphi$ by -1 in the above expression. The following commutator estimates hold:

Lemma 3.1 For $1 \leq|\alpha| \leq m, i=0,1,2,3$, one has that

$$
\int_{0}^{t}\left\|\mathcal{C}_{i}^{\alpha}(f)\right\|^{2} d \tau \lesssim \Lambda\left(\frac{1}{c_{0}},|h|_{\mathcal{W}_{t}^{2, \infty}}+\|\nabla f\|_{\mathcal{W}_{t}^{1, \infty}}\right) \int_{0}^{t}\|\nabla f\|_{\mathcal{H}^{m-1}}^{2}+\left|\mathcal{Z}^{m-1} h\right|_{\frac{1}{2}}^{2} d \tau .
$$

Proof. The proof is similar to 32 which involves only spacial norms, since the role of $Z_{0}=\partial_{t}$ is the same to tangential derivatives. The details are omitted here.

\subsection{Equations satisfied by $\left(\mathcal{Z}^{\alpha} v, \mathcal{Z}^{\alpha} p, \mathcal{Z}^{\alpha} \eta\right)$}

We now derive the system satisfied by $\mathcal{Z}^{\alpha} v, \mathcal{Z}^{\alpha} p, \mathcal{Z}^{\alpha} \eta$. Alihnac's good unknown are defined as

$$
V^{\alpha}=\mathcal{Z}^{\alpha} v-\partial_{z}^{\varphi} v \mathcal{Z}^{\alpha} \eta \text {, and } Q^{\alpha}=\mathcal{Z}^{\alpha} p-\partial_{z}^{\varphi} p \mathcal{Z}^{\alpha} \eta
$$

It should be noted that the following computation work only in a neighborhood of the boundary, but, for notational convenience, we write it in a general form without confusion.

Lemma 3.2 For $1 \leq|\alpha| \leq m, V^{\alpha}$ and $Q^{\alpha}$ satisfy

$$
\begin{aligned}
& \operatorname{div}^{\varphi} V^{\alpha}+\frac{1}{\gamma p} \partial_{t}^{\varphi} Q^{\alpha}+\frac{1}{\gamma p} v \cdot \nabla^{\varphi} Q^{\alpha} \\
& =\mathcal{Z}^{\alpha} \eta\left(\partial_{z}^{\varphi}\left(\frac{1}{\gamma p}\right) \partial_{t}^{\varphi} p+\partial_{z}^{\varphi}\left(\frac{v}{\gamma p}\right) \cdot \nabla^{\varphi} p\right)-\mathcal{C}^{\alpha}(d)-\frac{1}{\gamma p} \mathcal{C}_{0}^{\alpha}(p)-\frac{v}{\gamma p} \cdot \mathcal{C}^{\alpha}(p) \\
& \quad-\left[\mathcal{Z}^{\alpha}, \frac{1}{\gamma p}\right] \partial_{t}^{\varphi} p-\left[\mathcal{Z}^{\alpha}, \frac{v}{\gamma p}\right] \cdot \nabla^{\varphi} p \\
& =: \mathcal{R}_{d}^{\alpha},
\end{aligned}
$$


and

$$
\begin{aligned}
& \varrho\left(\partial_{t}^{\varphi}+v \cdot \nabla^{\varphi}\right) V^{\alpha}+\nabla^{\varphi} Q^{\alpha}-2 \mu \varepsilon d i v^{\varphi} S^{\varphi} V^{\alpha}-\lambda \varepsilon \nabla^{\varphi} d i v^{\varphi} V^{\alpha} \\
& =\mathcal{Z}^{\alpha} \eta \partial_{z}^{\varphi} \varrho\left(\partial_{t}^{\varphi} v+v \cdot \nabla^{\varphi} v\right)+\varrho \mathcal{Z}^{\alpha} \eta\left(\partial_{z}^{\varphi} v \cdot \nabla^{\varphi}\right) v-\left[\mathcal{Z}^{\alpha}, \varrho\right]\left(\partial_{t}^{\varphi} v+v \cdot \nabla^{\varphi} v\right)-\mathcal{C}^{\alpha}(p) \\
& \quad-\varrho \mathcal{C}^{\alpha}(T)+2 \mu \varepsilon d i v^{\varphi} \mathcal{E}^{\alpha}(v)+2 \mu \varepsilon \mathcal{D}^{\alpha}\left(S^{\varphi} v\right)+\lambda \varepsilon \nabla^{\varphi} \mathcal{C}^{\alpha}(d)+\lambda \varepsilon \mathcal{C}^{\alpha}\left(\operatorname{div}^{\varphi} v\right) \\
& =: \\
& \mathcal{R}_{M}^{\alpha},
\end{aligned}
$$

where the commutators $\mathcal{C}_{0}^{\alpha}(p), \mathcal{C}^{\alpha}(p), \mathcal{C}^{\alpha}(d), \mathcal{C}^{\alpha}(T)$ and $\mathcal{E}^{\alpha}(v)$ admit the estimates:

$$
\begin{aligned}
& \int_{0}^{t}\left\|\left(\mathcal{C}_{0}^{\alpha}(p), \mathcal{C}^{\alpha}(p)\right)\right\|^{2} d \tau \leq \Lambda\left(\frac{1}{c_{0}},|h|_{\mathcal{W}_{t}^{2, \infty}}+\|\nabla p\|_{\mathcal{W}_{t}^{1, \infty}}\right) \int_{0}^{t}\|\nabla p\|_{\mathcal{H}^{m-1}}^{2}+\left|\mathcal{Z}^{m-1} h\right|_{\frac{1}{2}}^{2} d \tau, \\
& \int_{0}^{t}\left\|\mathcal{C}^{\alpha}(d)\right\|^{2}+\left\|\mathcal{E}^{\alpha}(v)\right\|^{2} d \tau \leq \Lambda\left(\frac{1}{c_{0}},|h|_{\mathcal{W}_{t}^{2, \infty}}+\|\nabla v\|_{\mathcal{W}_{t}^{1, \infty}}\right) \int_{0}^{t}\|\nabla v\|_{\mathcal{H}^{m-1}}^{2}+\left|\mathcal{Z}^{m-1} h\right|_{\frac{1}{2}}^{2} d \tau, \\
& \int_{0}^{t}\left\|\mathcal{C}^{\alpha}(T)\right\|^{2} d \tau \leq \Lambda\left(\frac{1}{c_{0}},\|(v, \nabla v)\|_{\mathcal{W}_{t}^{1, \infty}}+|h|_{\mathcal{W}_{t}^{2, \infty}}\right) \int_{0}^{t}\|v\|_{\mathcal{H}^{m}}^{2}+\|\nabla v\|_{\mathcal{H}^{m-1}}^{2}+\left|\mathcal{Z}^{m} h\right|_{L^{2}}^{2} d \tau,
\end{aligned}
$$

and

$$
\begin{aligned}
& \int_{0}^{t}\left\|\left(\operatorname{div}^{\varphi} \mathcal{E}^{\alpha}(v), \mathcal{D}^{\alpha}\left(S^{\varphi} v\right), \nabla^{\alpha} \mathcal{C}^{\alpha}(d), \mathcal{C}^{\alpha}\left(d i v^{\varphi} v\right)\right)\right\|^{2} d \tau \\
& \leq \Lambda\left(\frac{1}{c_{0}},|h|_{\mathcal{W}_{t}^{3, \infty}}+\|\nabla v\|_{\mathcal{W}_{t}^{1, \infty}}+\sqrt{\varepsilon}\left\|\nabla^{2} v\right\|_{L_{t}^{\infty}}\right) \int_{0}^{t} \varepsilon^{2}\left\|\nabla^{2} v\right\|_{\mathcal{H}^{m-1}}^{2}+\|\nabla v\|_{\mathcal{H}^{m-1}}^{2}+\varepsilon^{2}\left|\nabla_{y} \mathcal{Z}^{m-1} h\right|_{\frac{1}{2}}^{2} d \tau
\end{aligned}
$$

Proof. It follows from (3.1) that

$$
\begin{aligned}
& \mathcal{Z}^{\alpha} \operatorname{div}^{\varphi} v=\operatorname{div}^{\varphi} \mathcal{Z}^{\alpha} v-\partial_{z}^{\varphi} v \cdot \nabla^{\varphi} \mathcal{Z}^{\alpha} \eta+\mathcal{C}^{\alpha}(d), \\
& \mathcal{Z}^{\alpha}\left(\frac{1}{\gamma p} \partial_{t}^{\varphi} p\right)=\frac{1}{\gamma p}\left(\partial_{t}^{\varphi} \mathcal{Z}^{\alpha} p-\partial_{z}^{\varphi} p \partial_{t}^{\varphi} \mathcal{Z}^{\alpha} \eta+\mathcal{C}_{0}^{\alpha}(p)\right)+\left[\mathcal{Z}^{\alpha}, \frac{1}{\gamma p}\right] \partial_{t}^{\varphi} p, \\
& \mathcal{Z}^{\alpha}\left(\frac{v}{\gamma p} \cdot \nabla^{\varphi} p\right)=\frac{v}{\gamma p} \cdot\left(\nabla^{\varphi} \mathcal{Z}^{\alpha} p-\partial_{z}^{\varphi} p \nabla^{\varphi} \mathcal{Z}^{\alpha} \eta+\mathcal{C}^{\alpha}(p)\right)+\left[\mathcal{Z}^{\alpha}, \frac{v}{\gamma p}\right] \cdot \nabla^{\varphi} p,
\end{aligned}
$$

where $\mathcal{C}^{\alpha}(d)=\sum_{i=1}^{3} \mathcal{C}_{i}^{\alpha}\left(v_{i}\right), \mathcal{C}^{\alpha}(p)=\left(\mathcal{C}_{1}^{\alpha}(p), \mathcal{C}_{2}^{\alpha}(p), \mathcal{C}_{3}^{\alpha}(p)\right)^{t}$. It follows from Lemma 3.1 that

$$
\begin{aligned}
& \int_{0}^{t}\left\|\mathcal{C}^{\alpha}(d)\right\|^{2} d \tau \leq \Lambda\left(\frac{1}{c_{0}},|h|_{\mathcal{W}_{t}^{2, \infty}}+\|\nabla v\|_{\mathcal{W}_{t}^{1, \infty}}\right) \int_{0}^{t}\|\nabla v\|_{\mathcal{H}^{m-1}}^{2}+\left|\mathcal{Z}^{m-1} h\right|_{\frac{1}{2}}^{2} d \tau \\
& \int_{0}^{t}\left\|\left(\mathcal{C}_{0}^{\alpha}(p), \mathcal{C}^{\alpha}(p)\right)\right\|^{2} d \tau \leq \Lambda\left(\frac{1}{c_{0}},|h|_{\mathcal{W}_{t}^{2, \infty}}+\|\nabla p\|_{\mathcal{W}_{t}^{1, \infty}}\right) \int_{0}^{t}\|\nabla p\|_{\mathcal{H}^{m-1}}^{2}+\left|\mathcal{Z}^{m-1} h\right|_{\frac{1}{2}}^{2} d \tau
\end{aligned}
$$

which proves (3.7) and a part of (3.8). Adding (3.11)-(3.13) and using (1.31)

Note that

$$
\mathcal{Z}^{\alpha}\left(\varrho\left(\partial_{t}^{\varphi} v+v \cdot \nabla^{\varphi} v\right)\right)=\varrho \mathcal{Z}^{\alpha}\left(\partial_{t}^{\varphi} v+v \cdot \nabla^{\varphi} v\right)+\left[\mathcal{Z}^{\alpha}, \varrho\right]\left(\partial_{t}^{\varphi} v+v \cdot \nabla^{\varphi} v\right)
$$

Rewrite the transport operator $\partial_{t}^{\varphi}+v \cdot \nabla^{\varphi}$ as

$$
\partial_{t}^{\varphi}+v \cdot \nabla^{\varphi}=\partial_{t}+v_{y} \nabla_{y}+V_{z} \partial_{z}
$$

where

$$
V_{z}=\frac{1}{\partial_{z} \varphi} v_{z}=\frac{1}{\partial_{z} \varphi}\left(v \cdot \mathbf{N}-\partial_{t} \varphi\right)=\frac{1}{\partial_{z} \varphi}\left(v \cdot \mathbf{N}-\partial_{t} \eta\right),
$$

and $\mathbf{N}(t, y, z)$ defined as

$$
\mathbf{N}(t, y, z)=\left(-\partial_{1} \eta(t, y, z),-\partial_{2} \eta(t, y, z), 1\right)^{t} .
$$

is an extension of the outward normal vector to the boundary. Henceforth, we will use it and the outward normal vector to the boundary without confusion.

It follows that

$$
\mathcal{Z}^{\alpha}\left(\partial_{t}^{\varphi}+v \cdot \nabla^{\varphi}\right) v=\left(\partial_{t}^{\varphi}+v \cdot \nabla^{\varphi}\right) \mathcal{Z}^{\alpha} v-\partial_{z}^{\varphi} v\left(\partial_{t}^{\varphi}+v \cdot \nabla^{\varphi}\right) \mathcal{Z}^{\alpha} \eta+\mathcal{C}^{\alpha}(T)
$$


where the commutator $\mathcal{C}^{\alpha}(T)$ is given by

$$
\mathcal{C}^{\alpha}(T)=\sum_{i=1}^{6} T_{i}^{\alpha}
$$

and

$$
\begin{aligned}
& T_{1}^{\alpha}=\left[\mathcal{Z}^{\alpha}, v_{y}\right] \nabla_{y} v, T_{2}^{\alpha}=\left[\mathcal{Z}^{\alpha}, V_{z}, \partial_{z} v\right], T_{3}^{\alpha}=\frac{\partial_{z} v}{\partial_{z} \varphi}\left(\left[\mathcal{Z}^{\alpha}, v_{y}\right] \nabla_{y} \eta+\mathcal{Z}^{\alpha} v_{z}\right), \\
& T_{4}^{\alpha}=\left(\mathcal{Z}^{\alpha}\left(\frac{1}{\partial_{z} \varphi}\right)+\frac{\mathcal{Z}^{\alpha} \partial_{z} \eta}{\left(\partial_{z} \varphi\right)^{2}}\right) v_{z} \partial_{z} v, T_{5}^{\alpha}=-\frac{v_{z} \partial_{z} v}{\left(\partial_{z} \varphi\right)^{2}}\left[\mathcal{Z}^{\alpha}, \partial_{z}\right] \eta+V_{z}\left[\mathcal{Z}^{\alpha}, \partial_{z}\right] v, T_{6}^{\alpha}=\left[\mathcal{Z}^{\alpha}, \frac{1}{\partial_{z} \varphi}, v_{z}\right] \partial_{z} v .
\end{aligned}
$$

Finally, for the viscous term, it follows from (3.1) that

$$
\mathcal{Z}^{\alpha} \operatorname{div}^{\varphi} S^{\varphi} v=\operatorname{div}^{\alpha} \mathcal{Z}^{\alpha} S^{\varphi} v-\partial_{z}^{\varphi} S^{\varphi} v \cdot \nabla^{\varphi} \mathcal{Z}^{\alpha} \eta+\mathcal{D}^{\alpha}\left(S^{\varphi} v\right)
$$

where $\mathcal{D}^{\alpha}\left(S^{\varphi} v\right)_{i}=\mathcal{C}_{j}^{\alpha}\left(S^{\varphi} v\right)_{i, j}$. Note that

$$
\mathcal{Z}^{\alpha} S^{\varphi} v=S^{\varphi} \mathcal{Z}^{\alpha} v-\partial_{z}^{\varphi} v \otimes \nabla^{\varphi} \mathcal{Z}^{\alpha} \eta-\nabla^{\varphi} \mathcal{Z}^{\alpha} \eta \otimes \partial_{z}^{\varphi} v+\mathcal{E}^{\alpha}(v),
$$

with $\left(\mathcal{E}^{\alpha} v\right)_{i j}=\mathcal{C}_{i}^{\alpha}\left(v_{j}\right)+\mathcal{C}_{j}^{\alpha}\left(v_{i}\right)$. Therefore, it follows from (3.18) and (3.19) that

$$
\begin{aligned}
\mathcal{Z}^{\alpha} \operatorname{div}^{\varphi} S^{\varphi} v= & \operatorname{div}^{\varphi} S^{\varphi} \mathcal{Z}^{\alpha} v-\operatorname{div}^{\varphi}\left(\partial_{z}^{\varphi} v \otimes \nabla^{\varphi} \mathcal{Z}^{\alpha} \eta+\nabla^{\varphi} \mathcal{Z}^{\alpha} \eta \otimes \partial_{z}^{\varphi} v\right) \\
& +\operatorname{div}^{\varphi} \mathcal{E}^{\alpha}(v)-\partial_{z}^{\varphi} S^{\varphi} v \cdot \nabla^{\varphi} \mathcal{Z}^{\alpha} \eta+2 \mu \varepsilon \mathcal{D}^{\alpha}\left(S^{\varphi} v\right) .
\end{aligned}
$$

Similarly, one has

$$
\begin{aligned}
\mathcal{Z}^{\alpha} \nabla^{\varphi} \operatorname{div}^{\varphi} v & =\nabla^{\varphi} \mathcal{Z}^{\alpha} \operatorname{div}^{\varphi} v-\partial_{z}^{\varphi} \operatorname{div}^{\varphi} v \nabla^{\varphi} \mathcal{Z}^{\alpha} \eta+\mathcal{C}^{\alpha}\left(\operatorname{div}^{\varphi} v\right) \\
& =\nabla^{\varphi} \operatorname{div}^{\varphi} \mathcal{Z}^{\alpha} v-\nabla^{\varphi}\left(\partial_{z}^{\varphi} v \cdot \nabla^{\varphi} \mathcal{Z}^{\alpha} \eta\right)-\partial_{z}^{\varphi} \operatorname{div}^{\varphi} v \nabla^{\varphi} \mathcal{Z}^{\alpha} \eta+\nabla^{\alpha} \mathcal{C}^{\alpha}(d)+\mathcal{C}^{\alpha}\left(\operatorname{div}^{\varphi} v\right)
\end{aligned}
$$

where (3.11) has been used in the last equality. Collecting (3.14), (3.17), (3.20), (3.21) and using (1.31) 2 lead to (3.6). Then Lemma 3.1 yields that

$$
\int_{0}^{t}\left\|\mathcal{E}^{\alpha}(v)\right\|^{2} d \tau \lesssim \Lambda\left(\frac{1}{c_{0}},|h|_{\mathcal{W}_{t}^{2, \infty}}+\|\nabla v\|_{\mathcal{W}_{t}^{1, \infty}}\right) \int_{0}^{t}\|\nabla v\|_{\mathcal{H}^{m-1}}^{2}+\left|\mathcal{Z}^{m-1} h\right|_{\frac{1}{2}}^{2} d \tau
$$

and

$$
\begin{aligned}
& \int_{0}^{t}\left\|\left(\operatorname{div}^{\varphi} \mathcal{E}^{\alpha}(v), \mathcal{D}^{\alpha}\left(S^{\varphi} v\right), \nabla^{\alpha} \mathcal{C}^{\alpha}(d), \mathcal{C}^{\alpha}\left(\operatorname{div}^{\varphi} v\right)\right)\right\|^{2} d \tau \\
& \leq \Lambda\left(\frac{1}{c_{0}},|h|_{\mathcal{W}_{t}^{3, \infty}}+\|\nabla v\|_{\mathcal{W}_{t}^{1, \infty}}+\sqrt{\varepsilon}\left\|\nabla^{2} v\right\|_{L_{t}^{\infty}}\right) \int_{0}^{t} \varepsilon^{2}\left\|\nabla^{2} v\right\|_{\mathcal{H}^{m-1}}^{2}+\|\nabla v\|_{\mathcal{H}^{m-1}}^{2}+\varepsilon^{2}\left|\nabla_{y} \mathcal{Z}^{m-1} h\right|_{\frac{1}{2}}^{2} d \tau .
\end{aligned}
$$

It remains to estimate the commutator $\mathcal{C}^{\alpha}(T)$. One first needs some estimates of $v_{z}$ and $V_{z}$ which will be used later. It follows from the definition of $v_{z}$ and $V_{z}$ in (3.16) that

$$
\begin{aligned}
\left\|v_{z}\right\|_{\infty, t}+\left\|V_{z}\right\|_{\infty, t} \leq & \Lambda\left(\frac{1}{c_{0}},\|v\|_{\infty, t}+\|\nabla \eta\|_{\infty, t}+\left\|\partial_{t} \eta\right\|_{\infty, t}\right) \leq \Lambda\left(\frac{1}{c_{0}},\|v\|_{\infty, t}+|h|_{\mathcal{W}_{t}^{1, \infty}}\right) \\
\left\|\mathcal{Z} v_{z}\right\|_{\infty, t}+\left\|\mathcal{Z} V_{z}\right\|_{\infty, t} & \leq \Lambda\left(\frac{1}{c_{0}},\|\mathcal{Z} v \cdot \mathbf{N}+v \cdot \mathcal{Z} \mathbf{N}\|_{\infty, t}+\left\|\partial_{t} \mathcal{Z} \eta\right\|_{\infty, t}+\left\|v_{z}\right\|_{\infty, t}+\left\|\mathcal{Z} \partial_{z} \varphi\right\|_{\infty, t}\right) \\
& \leq \Lambda\left(\frac{1}{c_{0}},\|v\|_{\mathcal{W}_{t}^{1, \infty}}+|h|_{\mathcal{W}_{t}^{2, \infty}}\right)
\end{aligned}
$$

Using (2.1) and Lemma 2.4 one has that

$$
\begin{aligned}
& \int_{0}^{t}\left\|\mathcal{Z} v_{z}\right\|_{\mathcal{H}^{m-2}}^{2} d \tau \lesssim \int_{0}^{t}\|\eta\|_{\mathcal{H}^{m}}^{2}+\|v \cdot \mathbf{N}\|_{\mathcal{H}^{m-1}}^{2} d \tau \\
& \leq \Lambda\left(\frac{1}{c_{0}},\|v\|_{\infty, t}+|h|_{\mathcal{W}_{t}^{1, \infty}}\right) \int_{0}^{t}\|v\|_{\mathcal{H}^{m-1}}^{2}+|h|_{\mathcal{H}^{m}}^{2} d \tau .
\end{aligned}
$$


It follows from Lemma 2.5 that

$$
\begin{aligned}
& \int_{0}^{t}\left\|\mathcal{Z} V_{z}\right\|_{\mathcal{H}^{m-2}}^{2} d \tau \leq \int_{0}^{t}\left\|\frac{1}{\partial_{z} \varphi} \mathcal{Z} v_{z}\right\|_{\mathcal{H}^{m-2}}^{2}+\left\|v_{z} \mathcal{Z}\left(\frac{1}{\partial_{z} \varphi}\right)\right\|_{\mathcal{H}^{m-2}}^{2} d \tau \\
& \leq \Lambda\left(\frac{1}{c_{0}},\left\|v_{z}\right\|_{\mathcal{W}_{t}^{1, \infty}}+|h|_{\mathcal{W}_{t}^{2, \infty}}\right) \int_{0}^{t}\left\|\left(v_{z}, \mathcal{Z} v_{z}\right)\right\|_{\mathcal{H}^{m-2}}^{2}+\left|\mathcal{Z}^{m-2} h\right|_{\frac{1}{2}}^{2} d \tau+\left\|\frac{\mathcal{Z} \partial_{z} \eta}{\left(\partial_{z} \varphi\right)^{2}}\right\|_{\mathcal{H}^{m-2}}^{2} d \tau \\
& \leq \Lambda\left(\frac{1}{c_{0}},\|v\|_{\mathcal{W}_{t}^{1, \infty}}+|h|_{\mathcal{W}_{t}^{2, \infty}}\right) \int_{0}^{t}\|v\|_{\mathcal{H}^{m-1}}^{2}+|h|_{\mathcal{H}^{m}}^{2} d \tau
\end{aligned}
$$

As a consequence of Lemma 2.1 and the above estimates for $v_{z}, V_{z}$, one gets that

$$
\int_{0}^{t}\left\|\mathcal{C}^{\alpha}(T)\right\|^{2} d \tau \leq \Lambda\left(\frac{1}{c_{0}},\|(v, \nabla v)\|_{\mathcal{W}_{t}^{1, \infty}}+|h|_{\mathcal{W}_{t}^{2, \infty}}\right) \int_{0}^{t}\|v\|_{\mathcal{H}^{m}}^{2}+\|\nabla v\|_{\mathcal{H}^{m-1}}^{2}+|h|_{\mathcal{H}^{m}}^{2} d \tau
$$

Therefore, the proof of Lemma 3.2 is completed.

\subsection{Boundary conditions for $\left(\mathcal{Z}^{\alpha} v, \mathcal{Z}^{\alpha} p, \mathcal{Z}^{\alpha} \eta\right)$}

In the following, we derive the boundary conditions for $\left(\mathcal{Z}^{\alpha} v, \mathcal{Z}^{\alpha} p, \mathcal{Z}^{\alpha} \eta\right)$. Note that the only interesting case occurs when $\alpha_{3}=0$, since $\mathcal{Z}^{\alpha} v=\mathcal{Z}^{\alpha} \eta=\mathcal{Z}^{\alpha} p=0$ with $\alpha_{3} \neq 0$ on the boundary. We start with the dynamic boundary condition.

Lemma 3.3 Let $1 \leq|\alpha| \leq m$ with $\alpha_{3}=0$, on the boundary $\{z=0\}$, it holds that

$$
\begin{aligned}
& \left(2 \mu \varepsilon S^{\varphi} V^{\alpha}+\lambda \varepsilon d i v^{\varphi} V^{\alpha}\right) \mathbf{N}+\mathcal{Z}^{\alpha} h\left(2 \mu \varepsilon \partial_{z}^{\varphi} S^{\varphi} v+\lambda \varepsilon \partial_{z}^{\varphi} d i v^{\varphi} v\right) \mathbf{N}-\sigma \nabla_{y} \cdot \mathcal{Z}^{\alpha}\left(\frac{\nabla_{y} h}{\sqrt{1+\left|\nabla_{y} h\right|^{2}}}\right) \mathbf{N} \\
& -\mathcal{Z}^{\alpha} p \mathbf{N}+\left(2 \mu \varepsilon S^{\varphi} v+\lambda \varepsilon d i v^{\varphi} v-\sigma \nabla_{y} \cdot \frac{\nabla_{y} h}{\sqrt{1+\left|\nabla_{y} h\right|^{2}}}-\left(p-p_{e}\right)\right) \mathcal{Z}^{\alpha} \mathbf{N}=\mathcal{C}^{\alpha}(B),
\end{aligned}
$$

where the commutator $\mathcal{C}^{\alpha}(B)$ satisfies

$$
\int_{0}^{t}\left|\mathcal{C}^{\alpha}(B)\right|_{L^{2}}^{2} d \tau \leq \Lambda\left(\frac{1}{c_{0}},|h|_{\mathcal{W}_{t}^{2, \infty}}+\|\nabla v\|_{\mathcal{W}_{t}^{1, \infty}}\right) \varepsilon^{2} \int_{0}^{t}\left|(\nabla v)^{b}\right|_{\mathcal{H}^{m-1}}^{2}+\left|\nabla_{y} h\right|_{\mathcal{H}^{m-1}}^{2} d \tau
$$

Here and henceforth, ()$^{b}$ denotes the value of function on the boundary $z=0$.

Remark 3.4 It should be emphasized that the (3.26) holds only locally on the boundary. But, this is enough since one can assume that the solution $(\varrho, v)$ is supported compactly on a neighborhood of the boundary. While, for the interior domain the boundary condition will not be involved in the conormal estimates.

Proof. Applying the operator $\mathcal{Z}^{\alpha}$ to (1.33) and using (3.11), (3.19), and the Alinhac good unknowns, one obtains (3.26) with the commutator $\mathcal{C}^{\alpha}(B)$ of the form

$$
\mathcal{C}^{\alpha}(B)=\left[\mathcal{Z}^{\alpha}, p-p_{e}+\sigma \nabla_{y} \cdot \frac{\nabla_{y} h}{\sqrt{1+\left|\nabla_{y} h\right|^{2}}}-2 \mu \varepsilon S^{\varphi} v-\lambda \varepsilon \operatorname{div}^{\varphi} v, \mathbf{N}\right]-2 \mu \varepsilon \mathcal{E}^{\alpha}(v)-\lambda \varepsilon \mathcal{C}^{\alpha}(d) .
$$

In order to control $\mathcal{C}^{\alpha}(B)$, we first notice, from (1.33), that

$$
p-p_{e}+\sigma \nabla_{y} \cdot \frac{\nabla_{y} h}{\sqrt{1+\left|\nabla_{y} h\right|^{2}}}=2 \mu \varepsilon\left(S^{\varphi} v \mathbf{n}\right) \cdot \mathbf{n}+\lambda \varepsilon d i v^{\varphi} v .
$$

which implies that

$$
\int_{0}^{t}\left|\mathcal{C}^{\alpha}(B)\right|_{L^{2}}^{2} d \tau \lesssim \varepsilon^{2} \int_{0}^{t}\left|\left[\mathcal{Z}^{\alpha}, \Pi\left(S^{\varphi} v\right), \mathbf{N}\right]\right|_{L^{2}\left(\mathbb{R}^{2}\right)}^{2}+\left|\mathcal{E}^{\alpha}(v)\right|_{L^{2}\left(\mathbb{R}^{2}\right)}^{2}+\left|\mathcal{C}^{\alpha}(d)\right|_{L^{2}\left(\mathbb{R}^{2}\right)}^{2} d \tau
$$


Here and henceforth, $\Pi:=\mathrm{Id}-\mathbf{n} \otimes \mathbf{n}$ denotes the tangential vector field. It follows from Lemma 2.1 that

$$
\begin{aligned}
\int_{0}^{t}\left|\left[\mathcal{Z}^{\alpha}, \Pi\left(S^{\varphi} v\right), \mathbf{N}\right]\right|_{L^{2}}^{2} d \tau & \lesssim \int_{0}^{t}\left\|\mathcal{Z} \Pi\left(S^{\varphi} v\right)\right\|_{\infty, t}^{2}|\mathcal{Z} \mathbf{N}|_{\mathcal{H}^{m-2}}^{2}+\|\mathcal{Z} \mathbf{N}\|_{\infty, t}^{2}\left|\mathcal{Z} \Pi\left(S^{\varphi} v\right)\right|_{\mathcal{H}^{m-2}}^{2} d \tau \\
& \lesssim \Lambda\left(\frac{1}{c_{0}},|h|_{\mathcal{W}_{t}^{2, \infty}}+\|\nabla v\|_{\mathcal{W}_{t}^{1, \infty}}\right) \int_{0}^{t}\left|\nabla_{y} h\right|_{\mathcal{H}^{m-1}}^{2}+\left|\nabla v^{b}\right|_{\mathcal{H}^{m-1}}^{2} d \tau
\end{aligned}
$$

To estimate $\mathcal{E}^{\alpha}(v)$ and $\mathcal{C}^{\alpha}(d)$ on the boundary, one needs only to control $\int_{0}^{t}\left|\mathcal{C}_{i}^{\alpha}\left(v_{j}\right)\right|_{L^{2}\left(\mathbb{R}^{2}\right)}^{2} d \tau$. Note that $\mathcal{C}_{i, 3}^{\alpha}\left(v_{j}\right)=0$ since we only consider the case that $\alpha_{3}=0$. Then, using the similar argument as in the proof of (3.3), one gets that

$$
\int_{0}^{t}\left|\mathcal{C}_{i}^{\alpha}\left(v_{j}\right)\right|^{2} d \tau=\Lambda\left(\frac{1}{c_{0}},|h|_{\mathcal{W}_{t}^{2, \infty}}+\|\nabla v\|_{\mathcal{W}_{t}^{1, \infty}}\right) \int_{0}^{t}\left|(\nabla v)^{b}\right|_{\mathcal{H}^{m-1}}^{2}+\left|\nabla_{y} h\right|_{\mathcal{H}^{m-1}}^{2} d \tau
$$

Substituting (3.29), (3.30) into (3.28), we prove (3.27). Therefore, the proof of Lemma 3.3 is completed.

Lemma 3.5 For any $1 \leq|\alpha| \leq m$ such that $\alpha_{3}=0$, on the boundary $\{z=0\}$, it holds that

$$
\partial_{t} \mathcal{Z}^{\alpha} h-v^{b} \cdot \mathcal{Z}^{\alpha} \mathbf{N}-V^{\alpha} \cdot \mathbf{N}=\mathcal{C}^{\alpha}(h),
$$

where the commutator $\mathcal{C}^{\alpha}(h)$ satisfies the estimate

$$
\int_{0}^{t}\left|\mathcal{C}^{\alpha}(h)\right|_{L^{2}}^{2} d \tau \leq \Lambda\left(\frac{1}{c_{0}},\|v\|_{\mathcal{W}_{t}^{1, \infty}}+\|\nabla v\|_{\infty, t}+|h|_{\mathcal{W}_{t}^{2, \infty}}\right) \int_{0}^{t}|h|_{\mathcal{H}^{m}}^{2}+\left|v_{y}^{b}\right|_{\mathcal{H}^{m-1}}^{2} .
$$

Proof. Applying the operator $\mathcal{Z}^{\alpha}$ to the kinematic boundary condition (1.32) ${ }_{1}$ shows that

$$
\partial_{t} \mathcal{Z}^{\alpha} h-v^{b} \cdot \mathcal{Z}^{\alpha} \mathbf{N}-V^{\alpha} \cdot \mathbf{N}=-\left[\mathcal{Z}^{\alpha}, v_{y}^{b}, \nabla_{y} h\right]+\frac{\left(\partial_{z} v\right)^{b}}{\partial_{z} \varphi} \mathcal{Z}^{\alpha} h \mathbf{N}:=\mathcal{C}^{\alpha}(h) .
$$

It follows from Lemma 2.1 that

$$
\int_{0}^{t}\left|\mathcal{C}^{\alpha}(h)\right|_{L^{2}}^{2} d \tau \leq \Lambda\left(\frac{1}{c_{0}},\|v\|_{\mathcal{W}_{t}^{1, \infty}}+\|\nabla v\|_{\infty, t}+|h|_{\mathcal{W}_{t}^{2, \infty}}\right) \int_{0}^{t}|h|_{\mathcal{H}^{m}}^{2}+\left|v_{y}^{b}\right|_{\mathcal{H}^{m-1}}^{2},
$$

which complete the proof of this lemma.

\section{A priori Estimates}

The aim of this section is to derive the a priori estimates in Theorem 4.1, which is a crucial step to prove Theorem 1.5. We drop the superscript $\varepsilon$ throughout this section for notation convenience.

Theorem 4.1 (A priori estimates) Let $m$ be an integer satisfying $m \geq 6, \varepsilon \in(0,1], \sigma \in[0,1]$, and $(\rho, u, F)$ be sufficiently smooth solution, defined on $\left[0, T^{\varepsilon}\right]$, to (1.1), (1.5), (1.6), (1.8), (1.9) with the initial data satisfying (1.38) and (1.46). Then it holds that

$$
\left|\varrho_{0}(y, z)\right| \exp \left(-\int_{0}^{t}\left\|d i v^{\varphi} v(\tau)\right\|_{L^{\infty}} d \tau\right) \leq \varrho(y, z, t) \leq\left|\varrho_{0}(y, z)\right| \exp \left(\int_{0}^{t}\left\|d i v^{\varphi} v(\tau)\right\|_{L^{\infty}} d \tau\right), \forall t \in\left[0, T^{\varepsilon}\right]
$$

Furthermore, there exists a time $T_{a}>0$ independent of $\sigma$ and $\varepsilon$ such that the following a priori estimate holds:

$$
\begin{aligned}
\Theta_{m}(t)=\sup _{0 \leq \tau \leq t}\left(1+\|(p, v, h)(\tau)\|_{X_{m}^{\varepsilon}}^{2}\right)+\int_{0}^{t}\|\nabla p(\tau)\|_{\mathcal{H}^{m-1}}^{2}+\|\Delta p(\tau)\|_{\mathcal{H}^{2}}^{2}+\|\nabla v(\tau)\|_{\mathcal{H}^{m-1}}^{4} d \tau \\
\quad+\varepsilon \int_{0}^{t}\|\nabla v(\tau)\|_{\mathcal{H}^{m}}^{2}+\left\|\nabla^{2} v(\tau)\right\|_{\mathcal{H}^{m-2}}^{2} d \tau+\varepsilon^{2} \int_{0}^{t}\left\|\nabla^{2} v(\tau)\right\|_{\mathcal{H}^{m-1}}^{2} d \tau \\
\leq \Lambda\left(C_{0}, \tilde{C}_{0}\right), \quad \forall t \in\left[0, \min \left(T_{a}, T_{\varepsilon}\right)\right]
\end{aligned}
$$

where $T_{a}$ depends only on $C_{0}, d_{0}$ and $\tilde{C}_{0}$. 
The main difficulties in the proof of Theorem 4.1 are to derive higher order conormal energy estimates for $(p, v, h)$. To overcome these difficulties, we will apply the Alinhac good unknowns

$$
V^{\alpha}=\mathcal{Z}^{\alpha} v-\partial_{z}^{\varphi} v \mathcal{Z}^{\alpha} \eta, \quad Q^{\alpha}=\mathcal{Z}^{\alpha} p-\partial_{z}^{\varphi} p \mathcal{Z}^{\alpha} \eta, \quad \alpha \neq 0
$$

and for $\alpha=0$, we denote $V^{0}=v$ and $Q^{0}=p$. As emphasized before, the good unknowns are chosen so that the corresponding commutators in the equations for $V^{\alpha}$ and $Q^{\alpha}$, which are computed in the previous section, are all lower order terms. The control of these good unknowns and $\mathcal{Z}^{\alpha} h$, which can be obtained by the standard energy method, yields a control of $v, p$ and $h$. Set

$$
\left\|V^{m}(t)\right\|^{2}=\sum_{|\alpha| \leq m}\left\|V^{\alpha}(t)\right\|^{2}, \quad\left\|\nabla V^{m}(t)\right\|^{2}=\sum_{|\alpha| \leq m}\left\|\nabla V^{\alpha}(t)\right\|^{2}, \quad\left\|Q^{m}(t)\right\|^{2}=\sum_{|\alpha| \leq m}\left\|Q^{\alpha}(t)\right\|^{2} .
$$

Throughout this section, we assume the following a priori assumptions hold:

$$
0<\frac{1}{4 C_{0}} \leq \varrho(t) \leq 4 C_{0}, \partial_{z} \varphi \geq c_{0},|h|_{\mathcal{H}^{3, \infty}}+\left|\nabla_{y} h\right|_{\mathcal{H}^{\left[\frac{m}{2}\right]+1}} \leq \frac{1}{c_{0}}, \quad-\left.\partial_{z}^{\varphi} p\right|_{z=0} \geq \frac{c_{0}}{2}, \forall t \in\left[0, T^{\varepsilon}\right]
$$

Thus, by using the assumption (4.0.4), one has that

$$
\begin{aligned}
\|v(t)\|_{\mathcal{H}^{m}}^{2} & \leq\left\|V^{m}(t)\right\|^{2}+\sum_{|\alpha| \leq m}\left\|\partial_{z}^{\varphi} v(t) \mathcal{Z}^{\alpha} \eta(t)\right\|^{2} \leq\left\|V^{m}(t)\right\|^{2}+\Lambda\left(\frac{1}{c_{0}},\|\nabla v\|_{L^{\infty}}\right)|h|_{\mathcal{H}^{m}}^{2}, \\
\|p(t)\|_{\mathcal{H}^{m}}^{2} & \leq\left\|Q^{m}(t)\right\|^{2}+\sum_{|\alpha| \leq m}\left\|\partial_{z}^{\varphi} p(t) \mathcal{Z}^{\alpha} \eta(t)\right\|^{2} \leq\left\|Q^{m}(t)\right\|^{2}+\Lambda\left(\frac{1}{c_{0}},\|\nabla p\|_{L^{\infty}}\right)|h|_{\mathcal{H}^{m}}^{2} .
\end{aligned}
$$

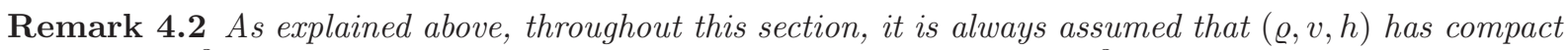
support in $\mathbb{R}^{3}$ or in a vicinity of $\partial \mathcal{S}$, and the system (1.31) holds in $\mathbb{R}^{3}$ or $\mathcal{S}$ because one can realize this assumption by multiplying a cut-off function and at the cost of some source terms (which are easy to estimate in the conormal Sobolev space because at least one derivative is applied to the cut-off function) appearing in the right hand side of (1.31).

Throughout this section, we shall work on the interval of time $\left[0, T^{\varepsilon}\right]$ such that (4.0.4) holds. And we point out that the generic constant $C$ may depend on $\frac{1}{c_{0}}, \mu$ and $\lambda$ in this section. Since the proof of Theorem 4.1 is very complicated, we divide the proof into the following subsections.

\subsection{Basic energy estimate}

First, we give the basic energy estimate which corresponds to the physical energy. Since it is not necessary to use the local coordinates to prove the basic energy estimate for (1.1), we use the Euler coordinates here. Precisely, the following lemma holds:

Lemma 4.3 For a smooth solution to (1.1), (1.5) and (1.6), it holds that

$$
\begin{aligned}
& \int_{\Omega_{t}} \frac{1}{2} \rho u^{2}+\frac{p(\rho)}{\gamma-1} d x+p_{e}\left|\Omega_{t}\right|+\sigma\left|\Sigma_{t}\right|+\int_{0}^{t} \int_{\Omega_{\tau}} 2 \mu \varepsilon|S u|^{2}+\lambda \varepsilon|d i v u|^{2} d x d \tau \\
& \leq \int_{\Omega_{0}} \frac{1}{2} \rho_{0} u_{0}^{2}+\frac{p\left(\rho_{0}\right)}{\gamma-1} d x+p_{e}\left|\Omega_{0}\right|+\sigma\left|\Sigma_{0}\right| .
\end{aligned}
$$

where $\left|\Omega_{t}\right|,\left|\Sigma_{t}\right|$ denote the volume of $\Omega_{t}$ and surface area of $\Sigma_{t}$, respectively.

Proof. Multiplying $(1.1)_{2}$ by $u$ and integrating over $\Omega_{t}$, one gets from integration by parts and the kinetic boundary condition (1.5) that

$$
\begin{aligned}
0= & \frac{d}{d t} \int_{\Omega_{t}} \frac{1}{2} \rho u^{2} d x-\int_{\Omega_{t}} p \operatorname{div} u d x+2 \mu \varepsilon \int_{\Omega_{t}}|S u|^{2} d x+\lambda \varepsilon \int_{\Omega_{t}}|\operatorname{div} u|^{2} d x \\
& +\int_{\Sigma_{t}}(p \mathbf{n}-2 \mu \varepsilon S u \mathbf{n}-\lambda \varepsilon \operatorname{div} u \mathbf{n}) \cdot u d S .
\end{aligned}
$$


where we have used the mass equation $[1.1)_{1}$ and the transport formula. The dynamic boundary condition (1.6) yields that

$$
\int_{\Sigma_{t}}(p \mathbf{n}-2 \mu \varepsilon S u \mathbf{n}-\lambda \varepsilon \operatorname{div} u \mathbf{n}) \cdot u d S=\int_{\Sigma_{t}}\left(p_{e} \mathbf{n}-\sigma H \mathbf{n}\right) \cdot u d S=\int_{\Omega_{t}} p_{e} \operatorname{div} u d x-\sigma \int_{\Sigma_{t}} H \mathbf{n} \cdot u d S .
$$

Using the mass equation (1.1) 1 and the transport formula, one obtains that

$$
-\int_{\Omega_{t}} p \operatorname{div} u d x+\int_{\Omega_{t}} p_{e} \operatorname{div} u d x=\frac{d}{d t} \int_{\Omega_{t}} \frac{\rho^{\gamma}}{\gamma-1} d x+\frac{d}{d t} \int_{\Omega_{t}} p_{e} d x .
$$

Note that the mean curvature of the free surface is give by (1.7). Let $\Sigma_{t}$ be determined by a local coordinate system $x=x\left(s_{1}, s_{2}, t\right),\left(s_{1}, s_{2}\right) \in U \subset \mathbb{R}^{2}$ so that its Riemannian metric given by

$$
d s=g_{\alpha \beta} d x^{\alpha} d x^{\beta},
$$

where $U$ is an open set, $g_{\alpha \beta}=x_{\alpha} \cdot x_{\beta}$ with $x_{\alpha}=\partial_{s^{\alpha}} x$ and the convention summation over the repeated indices has been used. It is well known that the Laplace-Beltrami operator can be written as

$$
\Delta_{\Sigma_{t}}(t)=\frac{1}{\sqrt{g}} \partial_{s^{\alpha}}\left(\sqrt{g} g^{\alpha \beta} \partial_{s^{\beta}}\right), \quad \alpha, \beta=1,2,
$$

with $g=\operatorname{det}_{\alpha \beta}$ and $\left(g^{\alpha \beta}\right)=\left(g_{\alpha \beta}\right)^{-1}$. Then, one gets that

$$
\begin{aligned}
& -\sigma \int_{\Sigma_{t}} H \mathbf{n} \cdot u d S=-\sigma \int_{\Sigma_{t}} g^{-\frac{1}{2}} \partial_{s^{\alpha}}\left(\sqrt{g} g^{\alpha \beta} \partial_{s^{\beta}} x\right) \cdot u d S \\
= & -\sigma \int_{U} \partial_{s^{\alpha}}\left(\sqrt{g} g^{\alpha \beta} \partial_{s^{\beta}} x\right) \cdot x_{t} d s_{1} d s_{2}=\frac{\sigma}{2} \int_{U} \sqrt{g} g^{\alpha \beta} \partial_{t} g_{\alpha \beta} d s_{1} d s_{2} \\
= & \sigma \frac{d}{d t} \int_{U} \sqrt{g} d s_{1} d s_{2}=\sigma \frac{d}{d t} \int_{\partial \Omega_{t}} d S .
\end{aligned}
$$

Substituting (4.1.3)-(4.1.6) into (4.1.2), one gets that

$$
\frac{d}{d t}\left(\int_{\Omega_{t}} \frac{1}{2} \rho u^{2}+\frac{p(\rho)}{\gamma-1} d x+p_{e}\left|\Omega_{t}\right|+\sigma\left|\Sigma_{t}\right|\right)+\int_{\Omega_{t}} 2 \mu \varepsilon|S u|^{2}+\lambda \varepsilon|\operatorname{div} u|^{2} d x=0 .
$$

Integrating the above equation, we proved (4.1.1). Therefore, the proof of this lemma is completed.

\subsection{Higher order conormal energy estimates}

Next, we perform the standard $L^{2}$ energy estimates on the Alinhac good unknowns $V^{\alpha}$ and $Q^{\alpha}$ solved by (3.5), (3.6). However, a new difficulty shall be overcame when the effect of surface tension $(\sigma \neq 0)$ is taken into consideration. It seems difficult to bound the boundary term involving the highest order derivatives of $\sigma \nabla_{y} \cdot \frac{\nabla_{y} h}{\sqrt{1+\left|\nabla_{y} h\right|^{2}}}$ due to the less regularity of $h, v(\mathrm{cf}$.(1.63) $)$. We will use the third equation in the boundary condition (3.26) and the equation (3.6) to turn this boundary term back into the volume integral. On the other hand, since (1.62) is invalid for compressible flows, $\varepsilon^{2} \int_{0}^{t}\left\|\nabla^{2} v\right\|_{\mathcal{H}^{m-1}}^{2} d \tau$ will appear in the estimate of $V^{\alpha}$ and $Q^{\alpha}$, when one directly apply the trace estimates to terms involving $\varepsilon^{2} \int_{0}^{t}\left|\left(\partial_{z} v\right)^{b}\right|_{\mathcal{H}^{m-1}}^{2} d \tau(\mathrm{cf}$. Lemma 3.3). Fortunately, with a $\varepsilon^{2}$ there, this term can be bounded by the dissipation terms in the estimates for normal derivatives and $\operatorname{div}^{\varphi} v$ in the next steps. Precisely, one has the following lemma.

Lemma 4.4 For any $t \in\left[0, T^{\varepsilon}\right]$ and $m \geq 5$, it holds that

$$
\begin{aligned}
& \left\|V^{m}(t)\right\|^{2}+\left\|Q^{m}(t)\right\|^{2}+|h(t)|_{\mathcal{H}^{m}}^{2}+\sigma\left|\nabla_{y} h(t)\right|_{\mathcal{H}^{m}}^{2}+\varepsilon \int_{0}^{t}\left\|\nabla V^{m}\right\|^{2} d \tau \\
& \leq \Lambda\left(\frac{1}{c_{0}},\left\|V^{m}(0)\right\|^{2}+\left\|Q^{m}(0)\right\|^{2}+\|v(0)\|_{\mathcal{H}^{m}}^{2}+\sigma\left|\nabla_{y} h(0)\right|_{\mathcal{H}^{m}}^{2}+|h(0)|_{\mathcal{H}^{m}}^{2}\right)+\Lambda_{0} \delta \int_{0}^{t}\|\nabla p\|_{\mathcal{H}^{m-1}}^{2} d \tau \\
& \quad+\Lambda_{0} \delta \varepsilon^{2} \int_{0}^{t}\left\|\nabla^{2} v\right\|_{\mathcal{H}^{m-1}}^{2} d \tau+C_{\delta} \Lambda_{\infty}(t) \int_{0}^{t} \Lambda\left(Y_{m}(\tau)\right)+\|\nabla v\|_{\mathcal{H}^{m-1}}^{2}+\varepsilon\left|\mathcal{Z}^{m} h\right|_{\frac{1}{2}}^{2} d \tau
\end{aligned}
$$


where $\Lambda_{0}=\Lambda\left(\frac{1}{c_{0}}, C_{0}\right), \Lambda_{\infty}(t)=\Lambda\left(\frac{1}{c_{0}}, C_{0}, \mathcal{Q}(t)\right)$ and

$$
\begin{aligned}
\mathcal{Q}(t):= & \|(v, \nabla v)\|_{\mathcal{W}_{t}^{1, \infty}}+\|(p, \nabla p)\|_{\mathcal{W}_{t}^{1, \infty}}+|h|_{\mathcal{W}_{t}^{3, \infty}}+\varepsilon^{\frac{1}{2}}\left\|\partial_{z z} v\right\|_{\infty, t}, \\
Y_{m}(t):= & \left\|\left(V^{m}, Q^{m}\right)(t)\right\|^{2}+\|\nabla(p, v)(t)\|_{\mathcal{H}^{m-2}}^{2}+\left|\left(h, \sqrt{\sigma} \nabla_{y} h\right)(t)\right|_{\mathcal{H}^{m}}^{2}+\left\|\Delta^{\varphi} p(t)\right\|_{\mathcal{H}^{1}}^{2} \\
& +\varepsilon\|\nabla(p, v)(t)\|_{\mathcal{H}^{m-1}}^{2}+\varepsilon\left\|\Delta^{\varphi} p(t)\right\|_{\mathcal{H}^{2}}^{2} .
\end{aligned}
$$

Proof. The case for $m=0$ is proved already in Lemma 4.3. Assume that (4.2.1) is proved for $k \leq m-1$. We shall prove that it holds for $k=m \geq 1$. Multiplying the equation (3.6) by $V^{\alpha}$ and integrating over the domain $\mathcal{S}$ yield

$$
\begin{aligned}
& \frac{d}{d t} \int_{\mathcal{S}} \frac{1}{2} \varrho\left|V^{\alpha}\right| d \mathcal{V}_{t}+\int_{\mathcal{S}} 2 \mu \varepsilon\left|S^{\varphi} V^{\alpha}\right|^{2}+\lambda \varepsilon\left|d i v^{\varphi} V^{\alpha}\right|^{2} d \mathcal{V}_{t} \\
& =\int_{z=0}\left(2 \mu \varepsilon S^{\varphi} V^{\alpha} \mathbf{N}+\lambda \varepsilon d i v^{\varphi} V^{\alpha} \mathbf{N}-Q^{\alpha} \mathbf{N}\right) V^{\alpha} d y+\int_{\mathcal{S}} Q^{\alpha} d i v^{\varphi} V^{\alpha} d \mathcal{V}_{t}+\int_{\mathcal{S}} \mathcal{R}_{M}^{\alpha} V^{\alpha} d \mathcal{V}_{t} \\
& =: \mathcal{B}+J+\int_{\mathcal{S}} \mathcal{R}_{M}^{\alpha} V^{\alpha} d \mathcal{V}_{t},
\end{aligned}
$$

where we have used the integration by parts and the boundary condition (1.32).

For the boundary term $\mathcal{B}$ in (4.2.4), it follows from (3.26) that

$$
\begin{aligned}
& \int_{0}^{t} \mathcal{B} d \tau=\sigma \int_{0}^{t} \int_{z=0} \nabla_{y} \cdot \mathcal{Z}^{\alpha}\left(\frac{\nabla_{y} h}{\sqrt{1+\left|\nabla_{y} h\right|^{2}}}\right) \mathbf{N} \cdot V^{\alpha} d y d \tau+\int_{0}^{t} \int_{z=0} \partial_{z}^{\varphi} p \mathcal{Z}^{\alpha} h \mathbf{N} \cdot V^{\alpha} d y d \tau \\
& -\int_{0}^{t} \int_{z=0}\left(2 \mu \varepsilon S^{\varphi} v+\lambda \varepsilon d i v^{\varphi} v-\sigma \nabla_{y} \cdot \frac{\nabla_{y} h}{\sqrt{1+\left|\nabla_{y} h\right|^{2}}}-\left(p-p_{e}\right)\right) \mathcal{Z}^{\alpha} \mathbf{N} \cdot V^{\alpha} d y d \tau \\
& -\int_{0}^{t} \int_{z=0} \mathcal{Z}^{\alpha} h\left(2 \mu \varepsilon \partial_{z}^{\varphi} S^{\varphi} v+\lambda \varepsilon \partial_{z}^{\varphi} d i v^{\varphi} v\right) \mathbf{N} V^{\alpha} d y d \tau+\int_{0}^{t} \int_{z=0} \mathcal{C}^{\alpha}(B) V^{\alpha} d y d \tau=: \sum_{i=1}^{5} K_{i}
\end{aligned}
$$

which will be dealt term by term. First, we deal with the most difficult one $K_{1}$. The kinetic boundary condition (3.31) yields

$$
K_{1}=\sigma \int_{0}^{t} \int_{z=0} \nabla_{y} \cdot \mathcal{Z}^{\alpha}\left(\frac{\nabla_{y} h}{\sqrt{1+\left|\nabla_{y} h\right|^{2}}}\right)\left(\partial_{t} \mathcal{Z}^{\alpha} h-v^{b} \cdot \mathcal{Z}^{\alpha} \mathbf{N}-\mathcal{C}^{\alpha}(h)\right) d y d \tau .
$$

Note that

$$
\mathcal{Z}^{\alpha} \frac{\nabla_{y} h}{\sqrt{1+\left|\nabla_{y} h\right|^{2}}}=\frac{\mathcal{Z}^{\alpha} \nabla_{y} h}{\sqrt{1+\left|\nabla_{y} h\right|^{2}}}-\frac{\nabla_{y} h\left\langle\nabla_{y} h, \mathcal{Z}^{\alpha} \nabla_{y} h\right\rangle}{\left(1+\left|\nabla_{y} h\right|^{2}\right)^{\frac{3}{2}}}+\mathcal{C}^{\alpha}(S),
$$

where $\mathcal{C}^{\alpha}(S)=\left[\mathcal{Z}^{\alpha}, \nabla_{y} h, \frac{1}{\sqrt{1+\left|\nabla_{y} h\right|^{2}}}\right]$. It follows from the integration by parts that

$$
\begin{aligned}
& \sigma \int_{0}^{t} \int_{z=0} \nabla_{y} \cdot\left(\frac{\mathcal{Z}^{\alpha} \nabla_{y} h}{\sqrt{1+\left|\nabla_{y} h\right|^{2}}}-\frac{\nabla_{y} h\left\langle\nabla_{y} h, \mathcal{Z}^{\alpha} \nabla_{y} h\right\rangle}{\left(1+\left|\nabla_{y} h\right|^{2}\right)^{\frac{3}{2}}}\right) \partial_{t} \mathcal{Z}^{\alpha} h d y d \tau \\
= & -\sigma \int_{0}^{t} \int_{z=0}\left(\frac{\mathcal{Z}^{\alpha} \nabla_{y} h}{\sqrt{1+\left|\nabla_{y} h\right|^{2}}}-\frac{\nabla_{y} h\left\langle\nabla_{y} h, \mathcal{Z}^{\alpha} \nabla_{y} h\right\rangle}{\left(1+\left|\nabla_{y} h\right|^{2}\right)^{\frac{3}{2}}}\right) \cdot \partial_{t} \mathcal{Z}^{\alpha} \nabla_{y} h d y d \tau \\
= & -\sigma \int_{0}^{t} \frac{d}{d \tau} \int_{z=0}\left(\frac{\left|\mathcal{Z}^{\alpha} \nabla_{y} h\right|^{2}}{2 \sqrt{1+\left|\nabla_{y} h\right|^{2}}}-\frac{\left|\left\langle\nabla_{y} h, \mathcal{Z}^{\alpha} \nabla_{y} h\right\rangle\right|^{2}}{2\left(1+\left|\nabla_{y} h\right|^{2}\right)^{\frac{3}{2}}}\right) d y d \tau \\
& +\sigma \int_{0}^{t} \int_{z=0}\left(-\frac{\left|\mathcal{Z}^{\alpha} \nabla_{y} h\right|^{2}\left\langle\nabla_{y} h, \partial_{t} \nabla_{y} h\right\rangle}{2\left(1+\left|\nabla_{y} h\right|^{2}\right)^{\frac{3}{2}}}+\frac{3\left|\left\langle\nabla_{y} h, \mathcal{Z}^{\alpha} \nabla_{y} h\right\rangle\right|^{2}\left\langle\nabla_{y} h, \partial_{t} \nabla_{y} h\right\rangle}{2\left(1+\left|\nabla_{y} h\right|^{2}\right)^{\frac{5}{2}}}\right) d y d \tau \\
& -\sigma \int_{0}^{t} \int_{z=0} \frac{\left\langle\nabla_{y} h, \mathcal{Z}^{\alpha} \nabla_{y} h\right\rangle\left\langle\partial_{t} \nabla_{y} h, \mathcal{Z}^{\alpha} \nabla_{y} h\right\rangle}{\left(1+\left|\nabla_{y} h\right|^{2}\right)^{\frac{3}{2}}} d y d \tau \\
\leq & -\sigma \int_{z=0} \frac{\left|\mathcal{Z}^{\alpha} \nabla_{y} h(t)\right|^{2}}{2\left(1+\left|\nabla_{y} h(t)\right|^{2}\right)^{\frac{3}{2}}} d y+\sigma \int_{z=0}\left(\frac{\left|\mathcal{Z}^{\alpha} \nabla_{y} h_{0}\right|^{2}}{2 \sqrt{1+\left|\nabla_{y} h_{0}\right|^{2}}}-\frac{\left|\left\langle\nabla_{y} h_{0}, \mathcal{Z}^{\alpha} \nabla_{y} h_{0}\right\rangle\right|^{2}}{2\left(1+\left|\nabla_{y} h_{0}\right|^{2}\right)^{\frac{3}{2}}}\right) d y \\
& +\Lambda_{\infty}(t) \sigma \int_{0}^{t}\left|\nabla_{y} h\right|_{\mathcal{H}^{m}}^{2} d \tau,
\end{aligned}
$$


and

$$
\begin{aligned}
& -\sigma \int_{0}^{t} \int_{z=0} \nabla_{y} \cdot\left(\frac{\mathcal{Z}^{\alpha} \nabla_{y} h}{\sqrt{1+\left|\nabla_{y} h\right|^{2}}}-\frac{\nabla_{y} h\left\langle\nabla_{y} h, \mathcal{Z}^{\alpha} \nabla_{y} h\right\rangle}{\left(1+\left|\nabla_{y} h\right|^{2}\right)^{\frac{3}{2}}}\right) v^{b} \cdot \mathcal{Z}^{\alpha} \mathbf{N} \\
= & \sigma \int_{0}^{t} \int_{z=0}\left(\frac{\mathcal{Z}^{\alpha} \nabla_{y} h}{\sqrt{1+\left|\nabla_{y} h\right|^{2}}}-\frac{\nabla_{y} h\left\langle\nabla_{y} h, \mathcal{Z}^{\alpha} \nabla_{y} h\right\rangle}{\left(1+\left|\nabla_{y} h\right|^{2}\right)^{\frac{3}{2}}}\right) \cdot \nabla_{y}\left(v^{b} \cdot \mathcal{Z}^{\alpha} \mathbf{N}\right) d y d \tau \\
\leq & \sigma \int_{0}^{t} \int_{z=0}\left(\frac{\mathcal{Z}^{\alpha} \nabla_{y} h}{\sqrt{1+\left|\nabla_{y} h\right|^{2}}}-\frac{\nabla_{y} h\left\langle\nabla_{y} h, \mathcal{Z}^{\alpha} \nabla_{y} h\right\rangle}{\left(1+\left|\nabla_{y} h\right|^{2}\right)^{\frac{3}{2}}}\right) \cdot\left(v_{y}^{b} \cdot \nabla_{y}\right) \mathcal{Z}^{\alpha} \nabla_{y} h d y d \tau \\
& +\sigma \int_{0}^{t} \int_{z=0}\left(\frac{\mathcal{Z}^{\alpha} \nabla_{y} h}{\sqrt{1+\left|\nabla_{y} h\right|^{2}}}-\frac{\nabla_{y} h\left\langle\nabla_{y} h, \mathcal{Z}^{\alpha} \nabla_{y} h\right\rangle}{\left(1+\left|\nabla_{y} h\right|^{2}\right)^{\frac{3}{2}}}\right) \cdot\left(\nabla_{y} v^{b} \cdot \mathcal{Z}^{\alpha} \mathbf{N}\right) d y d \tau \\
\leq & \sigma \int_{0}^{t} \int_{z=0} \frac{v_{y}^{b} \cdot \nabla_{y}\left(\left|\mathcal{Z}^{\alpha} \nabla_{y} h\right|^{2}\right)}{2 \sqrt{1+\left|\nabla_{y} h\right|^{2}}}-\frac{v_{y}^{b} \cdot \nabla_{y}\left(\left|\left\langle\nabla_{y} h, \mathcal{Z}^{\alpha} \nabla_{y} h\right\rangle\right|^{2}\right)}{2\left(1+\left|\nabla_{y} h\right|^{2}\right)^{\frac{3}{2}}} d y d \tau \\
& +\sigma \int_{0}^{t} \int_{z=0} \frac{\mathcal{Z}^{\alpha} \nabla_{y} h\left\langle\nabla_{y} h, \mathcal{Z}^{\alpha} \nabla_{y} h\right\rangle}{\left(1+\left|\nabla_{y} h\right|^{2}\right)^{\frac{3}{2}}}\left(v_{y}^{b} \cdot \nabla_{y}\right) \nabla_{y} h d y d \tau \\
& +\sigma \int_{0}^{t} \int_{z=0}\left(\frac{\mathcal{Z}^{\alpha} \nabla_{y} h}{\sqrt{1+\left|\nabla_{y} h\right|^{2}}}-\frac{\nabla_{y} h\left\langle\nabla_{y} h, \mathcal{Z}^{\alpha} \nabla_{y} h\right\rangle}{\left(1+\left|\nabla_{y} h\right|^{2}\right)^{\frac{3}{2}}}\right) \cdot\left(\nabla_{y} v_{y}^{b} \cdot \mathcal{Z}^{\alpha} \nabla_{y} h\right) d y d \tau \\
\leq & \Lambda_{\infty}(t) \sigma \int_{0}^{t}\left|\nabla_{y} h\right|_{\mathcal{H}^{m}}^{2} d \tau .
\end{aligned}
$$

Using Lemma 2.1, one has that

$$
\int_{0}^{t}\left|\mathcal{C}^{\alpha}(S)\right|_{L^{2}}^{2} d \tau \leq \Lambda_{\infty}(t) \int_{0}^{t}\left|\nabla_{y} h\right|_{\mathcal{H}^{m-1}}^{2} d \tau
$$

and

$$
\int_{0}^{t}\left|\partial_{t} \mathcal{C}^{\alpha}(S)\right|_{L^{2}}^{2}+\left|\nabla_{y} \mathcal{C}^{\alpha}(S)\right|_{L^{2}}^{2} d \tau \leq \Lambda_{\infty}(t) \int_{0}^{t}\left|\nabla_{y} h\right|_{\mathcal{H}^{m}}^{2} d \tau
$$

Therefore, from (4.2.10), (3.32) and trace estimate (2.5), one obtains that

$$
\begin{aligned}
& \left|\sigma \int_{0}^{t} \int_{z=0} \nabla_{y} \cdot \mathcal{C}^{\alpha}(S)\left(-v^{b} \cdot \mathcal{Z}^{\alpha} \mathbf{N}-\mathcal{C}^{\alpha}(h)\right) d y d \tau\right| \\
& \leq \sigma \Lambda_{\infty}(t)\left\{\int_{0}^{t}\left|\nabla_{y} \cdot \mathcal{C}^{\alpha}(S)\right|_{L^{2}}^{2}\right\}^{\frac{1}{2}}\left\{\int_{0}^{t}\left|\nabla_{y} h\right|_{\mathcal{H}^{m}}^{2}+\left|\mathcal{C}^{\alpha}(h)\right|^{2} d \tau\right\}^{\frac{1}{2}} \\
& \leq \Lambda_{\infty}(t) \int_{0}^{t}|h|_{\mathcal{H}^{m}}^{2}+\|v\|_{\mathcal{H}^{m-1}}^{2}+\sigma\left|\nabla_{y} h\right|_{\mathcal{H}^{m}}^{2}+\|\nabla v\|_{\mathcal{H}^{m-1}}^{2} d \tau
\end{aligned}
$$

For $\int_{0}^{t} \int_{z=0} \nabla_{y} \cdot \mathcal{C}^{\alpha}(S) \partial_{t} \mathcal{Z}^{\alpha} h d y d \tau$, if $\mathcal{Z}^{\alpha} \neq \partial_{t}^{m}$, then (4.2.10) yields immediately that

$$
\left|\sigma \int_{0}^{t} \int_{z=0} \nabla_{y} \cdot \mathcal{C}^{\alpha}(S) \partial_{t} \mathcal{Z}^{\alpha} h\right| \leq \Lambda_{\infty}(t) \sigma \int_{0}^{t} \mid \nabla_{y} h_{\mathcal{H}^{m}}^{2} d \tau .
$$

For $\mathcal{Z}^{\alpha}=\partial_{t}^{m}$, it follows from integration by parts and (4.2.10) that

$$
\begin{aligned}
& \left|\sigma \int_{0}^{t} \int_{z=0} \nabla_{y} \cdot \mathcal{C}^{\alpha}(S) \partial_{t}^{m+1} h d y d \tau\right|=\left|\sigma \int_{0}^{t} \int_{z=0} \mathcal{C}^{\alpha}(S) \partial_{t}^{m+1} \nabla_{y} h d y d \tau\right| \\
& \leq\left|\int_{z=0} \sigma \mathcal{C}^{\alpha}(S) \partial_{t}^{m} \nabla_{y} h d y\right|+\left|\sigma \int_{z=0} \mathcal{C}^{\alpha}\left(S_{0}\right) \partial_{t}^{m} \nabla_{y} h_{0} d y\right|+\left|\sigma \int_{0}^{t} \int_{z=0} \partial_{t} \mathcal{C}^{\alpha}(S) \partial_{t}^{m} \nabla_{y} h d y d \tau\right| \\
& \leq \Lambda_{0}\left[\left|\nabla_{y} h(0)\right|_{\mathcal{H}^{m-1}}^{2}+\sigma\left|\nabla_{y} h_{0}\right|_{\mathcal{H}^{m}}^{2}\right]+\frac{\sigma}{8} \int_{z=0} \frac{\left|\mathcal{Z}^{\alpha} \nabla_{y} h(t)\right|^{2}}{2\left(1+\left|\nabla_{y} h(t)\right|^{2}\right)^{\frac{3}{2}}} d y+\Lambda_{0} \sigma\left|\nabla_{y} h\right|_{\mathcal{H}^{m-1}}^{2} \\
& \quad+\Lambda_{\infty}(t) \sigma \int_{0}^{t}\left|\nabla_{y} h\right|_{\mathcal{H}^{m}}^{2} d \tau,
\end{aligned}
$$


where one has used that, for $m \geq 5$,

$$
\begin{aligned}
\left|\mathcal{C}^{\alpha}(S)(t)\right|_{L^{2}}^{2} & \lesssim \sum_{a=1}^{m-1}\left|\partial_{t}^{m-a} \nabla_{y} h \partial_{t}^{a} \frac{1}{\sqrt{1+\left|\nabla_{y} h\right|^{2}}}\right|_{L^{2}}^{2} \\
& \lesssim \sum_{a=1}^{\left[\frac{m}{2}\right]}\left|\partial_{t}^{a} \frac{1}{\sqrt{1+\left|\nabla_{y} h\right|^{2}}}\right|_{t, \infty}^{2}\left|\partial_{t}^{m-a} \nabla_{y} h\right|_{L^{2}}^{2}+\sum_{a=\left[\frac{m}{2}\right]+1}^{m-1}\left|\partial_{t}^{m-a} \nabla_{y} h\right|_{t, \infty}^{2}\left|\partial_{t}^{a} \frac{1}{\sqrt{1+\left|\nabla_{y} h\right|^{2}}}\right|_{L^{2}}^{2} \\
& \leq \Lambda\left(\frac{1}{c_{0}},\left|\nabla_{y} h\right|_{\mathcal{H}^{\left[\frac{m}{2}\right]+1}}^{2}\right)\left|\nabla_{y} h\right|_{\mathcal{H}^{m-1}}^{2} \leq \Lambda_{0}\left|\nabla_{y} h\right|_{\mathcal{H}^{m-1}}^{2}
\end{aligned}
$$

It should be reminded that the estimate of

$$
-\sigma \int_{0}^{t} \int_{z=0} \nabla_{y} \cdot\left(\frac{\mathcal{Z}^{\alpha} \nabla_{y} h}{\sqrt{1+\left|\nabla_{y} h\right|^{2}}}-\frac{\nabla_{y} h\left\langle\nabla_{y} h, \mathcal{Z}^{\alpha} \nabla_{y} h\right\rangle}{\left(1+\left|\nabla_{y} h\right|^{2}\right)^{\frac{3}{2}}}\right) \mathcal{C}^{\alpha}(h) d y d \tau
$$

is a subtle issue. If one uses directly the integration by parts, the term $\int_{0}^{t}\left|\nabla_{y} v^{b}\right|_{\mathcal{H}^{m-1}}^{2}$, which is difficult to estimate, must appear due to the boundary term $\mathcal{Z}^{\beta} v_{y}^{b} \cdot \mathcal{Z} \nabla_{y} h$ with $|\beta|=m-1$ in $\mathcal{C}^{\alpha}(h)$. Therefore, to deal with this boundary term, we will take full advantage of the boundary condition (3.26) and equation (3.6) to turn it into a volume integral. In fact, the dynamic boundary condition (3.26) yields that

$$
\begin{aligned}
& -\sigma \nabla_{y} \cdot \mathcal{Z}^{\alpha}\left(\frac{\nabla_{y} h}{\sqrt{1+\left|\nabla_{y} h\right|^{2}}}\right)-\partial_{z}^{\varphi} p \mathcal{Z}^{\alpha} h \\
= & Q^{\alpha}-\left(\left(2 \mu \varepsilon S^{\varphi} V^{\alpha}+\lambda \varepsilon \operatorname{div}^{\varphi} V^{\alpha}\right) \mathbf{N}\right)_{3}-\left(\mathcal{Z}^{\alpha} h\left(2 \mu \varepsilon \partial_{z}^{\varphi} S^{\varphi} v+\lambda \varepsilon \partial_{z}^{\varphi} \operatorname{div}^{\varphi} v\right) \mathbf{N}\right)_{3}+\mathcal{C}^{\alpha}(B)_{3} .
\end{aligned}
$$

where $(\cdot)_{3}$ denotes the third component of a vector. Thus, it follows from (3.27) and trace estimate (2.5) that

$$
\begin{aligned}
- & \sigma \int_{0}^{t} \int_{z=0} \nabla_{y} \cdot\left(\frac{\mathcal{Z}^{\alpha} \nabla_{y} h}{\sqrt{1+\left|\nabla_{y} h\right|^{2}}}-\frac{\nabla_{y} h\left\langle\nabla_{y} h, \mathcal{Z}^{\alpha} \nabla_{y} h\right\rangle}{\left(1+\left|\nabla_{y} h\right|^{2}\right)^{\frac{3}{2}}}\right) \mathcal{Z}^{\beta} v_{y}^{b} \cdot \mathcal{Z} \nabla_{y} h d y d \tau \\
\leq & \int_{0}^{t} \int_{z=0} Q^{\alpha} \mathcal{Z}^{\beta} v_{y}^{b} \cdot \mathcal{Z} \nabla_{y} h d y d \tau-\int_{0}^{t} \int_{z=0}\left(\left(2 \mu \varepsilon S^{\varphi} V^{\alpha}+\lambda \varepsilon \operatorname{div}^{\varphi} V^{\alpha}\right) \mathbf{N}\right)_{3} \mathcal{Z}^{\beta} v_{y}^{b} \cdot \mathcal{Z} \nabla_{y} h d y d \tau \\
& +\Lambda_{\infty}(t)\left(\int_{0}^{t}\left|v_{y}^{b}\right|_{\mathcal{H}^{m-1}}^{2} d \tau\right)^{\frac{1}{2}}\left(\int_{0}^{t}|h|_{\mathcal{H}^{m}}^{2}+\left|\mathcal{C}^{\alpha}(B)_{3}\right|_{L^{2}}^{2}+\sigma\left|\nabla_{y} \cdot \mathcal{C}^{\alpha}(S)\right|_{L^{2}}^{2} d \tau\right)^{\frac{1}{2}} \\
\leq & \int_{0}^{t} \int_{z=0} Q^{\alpha} \mathcal{Z}^{\beta} v_{y}^{b} \cdot \mathcal{Z} \nabla_{y} h d y d \tau-\int_{0}^{t} \int_{z=0}\left(\left(2 \mu \varepsilon S^{\varphi} V^{\alpha}+\lambda \varepsilon \operatorname{div}^{\varphi} V^{\alpha}\right) \mathbf{N}\right)_{3} \mathcal{Z}^{\beta} v_{y}^{b} \cdot \mathcal{Z} \nabla_{y} h d y d \tau \\
& +\delta \int_{0}^{t} \varepsilon^{2}\left\|\nabla^{2} v\right\|_{\mathcal{H}^{m-1}}^{2} d \tau+C_{\delta} \Lambda_{\infty}(t) \int_{0}^{t}|h|_{\mathcal{H}^{m}}^{2}+\|v\|_{\mathcal{H}^{m-1}}^{2}+\|\nabla v\|_{\mathcal{H}^{m-1}}^{2}+\sigma\left|\nabla_{y} h\right|_{\mathcal{H}^{m}}^{2} d \tau,
\end{aligned}
$$

where $|\beta|=m-1$. To estimate the boundary term $\int_{0}^{t} \int_{z=0} Q^{\alpha} \mathcal{Z}^{\beta} v_{y}^{b} \cdot \mathcal{Z} \nabla_{y} h d y d \tau$ in (4.2.17), using integration by parts, we note that

$$
\begin{aligned}
& \int_{0}^{t} \int_{z=0} Q^{\alpha} \mathcal{Z}^{\beta} v_{y}^{b} \cdot \mathcal{Z} \nabla_{y} h d y d \tau \\
& =\int_{0}^{t} \int_{\mathcal{S}} \partial_{z}^{\varphi} Q^{\alpha} \mathcal{Z}^{\beta} v_{y} \cdot \mathcal{Z} \nabla_{y} \eta d \mathcal{V}_{\tau} d \tau+\int_{0}^{t} \int_{\mathcal{S}} Q^{\alpha} \partial_{z}^{\varphi}\left(\mathcal{Z}^{\beta} v_{y} \cdot \mathcal{Z} \nabla_{y} \eta\right) d \mathcal{V}_{\tau} d \tau \\
& \leq \int_{0}^{t} \int_{\mathcal{S}} \partial_{z}^{\varphi} Q^{\alpha} \mathcal{Z}^{\beta} v_{y} \cdot \mathcal{Z} \nabla_{y} \eta d \mathcal{V}_{\tau} d \tau+\int_{0}^{t}\|\nabla v\|_{\mathcal{H}^{m-1}}^{2} d \tau+\Lambda_{\infty}(t) \int_{0}^{t}\|v\|_{\mathcal{H}^{m-1}}^{2}+\left\|Q^{m}\right\|^{2} d \tau
\end{aligned}
$$

Using the equation (3.6), one obtains that

$$
\begin{aligned}
& \int_{0}^{t} \int_{\mathcal{S}} \partial_{z}^{\varphi} Q^{\alpha} \mathcal{Z}^{\beta} v_{y} \cdot \mathcal{Z} \nabla_{y} \eta d \mathcal{V}_{\tau} d \tau \\
& \leq-\int_{0}^{t} \int_{\mathcal{S}} \varrho\left(\partial_{t}+v_{y} \cdot \nabla_{y}+V_{z} \partial_{z}\right)\left(V^{\alpha}\right)_{3} \mathcal{Z}^{\beta} v_{y} \cdot \mathcal{Z} \nabla_{y} \eta d \mathcal{V}_{\tau} d \tau \\
& \quad+\int_{0}^{t} \int_{\mathcal{S}}\left(2 \mu \varepsilon \operatorname{div}^{\varphi} S^{\varphi} V^{\alpha}+\lambda \varepsilon \nabla^{\varphi} \operatorname{div}^{\varphi} V^{\alpha}\right)_{3} \mathcal{Z}^{\beta} v_{y} \cdot \mathcal{Z} \nabla_{y} \eta d \mathcal{V}_{\tau} d \tau+\Lambda_{\infty}(t) \int_{0}^{t}\left\|\mathcal{R}_{M}^{\alpha}\right\|\|v\|_{\mathcal{H}^{m-1}} d \tau
\end{aligned}
$$


where $|\beta|=m-1$ and $\left\|\mathcal{R}_{M}^{\alpha}\right\|$ will be estimated later. Integration by parts, and using $V_{z}=0$ on $z=0$, one gets that

$$
\begin{aligned}
& \quad-\int_{0}^{t} \int_{\mathcal{S}} \varrho\left(\partial_{t}+v_{y} \cdot \nabla_{y}+V_{z} \partial_{z}\right)\left(V^{\alpha}\right)_{3} \mathcal{Z}^{\beta} v_{y} \cdot \mathcal{Z} \nabla_{y} \eta d \mathcal{V}_{\tau} d \tau \\
& =-\int_{\mathcal{S}} \varrho\left(V^{\alpha}\right)_{3} \mathcal{Z}^{\beta} v_{y} \cdot \nabla_{y} \eta d \mathcal{V}_{t}+\int_{\mathcal{S}} \varrho_{0}\left(V_{0}^{\alpha}\right)_{3} \mathcal{Z}^{\beta} v_{y}(0) \cdot \nabla_{y} \eta_{0} d \mathcal{V}_{0} \\
& +\Lambda_{\infty}(t) \int_{0}^{t}\left\|V^{\alpha}(\tau)\right\|^{2}+\|v(\tau)\|_{\mathcal{H}^{m}}^{2} d \tau \\
& \leq \frac{1}{16} \int \varrho\left|V^{\alpha}(t)\right|^{2} d \mathcal{V}_{t}+\Lambda_{0}\left[\left\|V^{\alpha}(0)\right\|^{2}+\|v(0)\|_{\mathcal{H}^{m-1}}^{2}\right]+\Lambda_{\infty}(t) \int_{0}^{t}\left\|V^{m}\right\|^{2}+\|v\|_{\mathcal{H}^{m}}^{2}+|h|_{\mathcal{H}^{m}}^{2} d \tau .
\end{aligned}
$$

For the second term in (4.2.19), it follows from the integrating by parts that

$$
\begin{aligned}
& \int_{0}^{t} \int_{\mathcal{S}}\left(2 \mu \varepsilon \operatorname{div}^{\varphi} S^{\varphi} V^{\alpha}+\lambda \varepsilon \nabla^{\varphi} \operatorname{div}^{\varphi} V^{\alpha}\right)_{3} \mathcal{Z}^{\beta} v_{y} \cdot \mathcal{Z} \nabla_{y} \eta d \mathcal{V}_{\tau} d \tau \\
& =-\int_{0}^{t} \int_{\mathcal{S}} \mu \varepsilon\left(\nabla^{\varphi} V_{3}^{\alpha}+\partial_{z}^{\varphi} V^{\alpha}\right) \cdot \nabla^{\varphi}\left(\mathcal{Z}^{\beta} v_{y} \cdot \mathcal{Z} \nabla_{y} \eta\right)+\lambda \varepsilon \operatorname{div}^{\varphi} V^{\alpha} \partial_{z}^{\varphi}\left(\mathcal{Z}^{\beta} v_{y} \cdot \mathcal{Z} \nabla_{y} \eta\right) d \mathcal{V}_{\tau} d \tau \\
& +\int_{0}^{t} \int_{z=0}\left(\left(2 \mu \varepsilon S^{\varphi} V^{\alpha}+\lambda \varepsilon \operatorname{div}^{\varphi} V^{\alpha}\right) \mathbf{N}\right)_{3} \mathcal{Z}^{\beta} v_{y}^{b} \cdot \mathcal{Z} \nabla_{y} h d y d \tau \\
& \leq \delta \varepsilon \int_{0}^{t}\left\|\nabla V^{m}\right\|^{2} d \tau+C_{\delta} \Lambda_{\infty}(t) \int_{0}^{t} \varepsilon\|\nabla v\|_{\mathcal{H}^{m-1}}^{2} \\
& +\int_{0}^{t} \int_{z=0}\left(\left(2 \mu \varepsilon S^{\varphi} V^{\alpha}+\lambda \varepsilon \operatorname{div}^{\varphi} V^{\alpha}\right) \mathbf{N}\right)_{3} \mathcal{Z}^{\beta} v_{y}^{b} \cdot \mathcal{Z} \nabla_{y} h d y d \tau .
\end{aligned}
$$

Combining (4.2.17)-(4.2.21) and noticing the cancellation between the worst boundary terms in (4.2.17) and (4.2.21), it follows from (4.0.5), for $|\beta|=m-1$, that

$$
\begin{aligned}
& -\sigma \int_{0}^{t} \int_{z=0} \nabla_{y} \cdot\left(\frac{\mathcal{Z}^{\alpha} \nabla_{y} h}{\sqrt{1+\left|\nabla_{y} h\right|^{2}}}-\frac{\nabla_{y} h\left\langle\nabla_{y} h, \mathcal{Z}^{\alpha} \nabla_{y} h\right\rangle}{\left(1+\left|\nabla_{y} h\right|^{2}\right)^{\frac{3}{2}}}\right) \mathcal{Z}^{\beta} v_{y}^{b} \cdot \mathcal{Z} \nabla_{y} h d y d \tau \\
& \leq \frac{1}{16} \int \varrho\left|V^{\alpha}(t)\right|^{2} d \mathcal{V}_{t}+C \delta \int_{0}^{t} \varepsilon^{2}\left\|\nabla^{2} v\right\|_{\mathcal{H}^{m-1}}^{2}+\varepsilon\left\|\nabla V^{m}\right\|_{\mathcal{H}^{m-1}}^{2} d \tau \\
& +\Lambda_{0}\left[\left\|V^{m}(0)\right\|^{2}+\|v(0)\|_{\mathcal{H}^{m-1}}^{2}\right]+C_{\delta} \Lambda_{\infty}(t) \int_{0}^{t} Y_{m}+\|\nabla v\|_{\mathcal{H}^{m-1}}^{2}+\left\|\mathcal{R}_{M}^{\alpha}\right\| Y_{m} d \tau .
\end{aligned}
$$

For the terms of the form $\mathcal{Z}^{\beta} v_{y} \mathcal{Z}^{\gamma} \nabla_{y} h$ with $|\beta|+|\gamma| \leq m, 1 \leq|\beta| \leq m-2$ and the term $\frac{\left(\partial_{z} v\right)^{b}}{\partial_{z} \varphi} \mathcal{Z}^{\alpha} h \mathbf{N}$ in $\mathcal{C}^{\alpha}(h)$, it follows from the integration by parts, Lemma 2.1, trace estimate (2.5) and (4.0.5) that

$$
\begin{aligned}
& -\sigma \int_{0}^{t} \int_{z=0} \nabla_{y} \cdot\left(\frac{\mathcal{Z}^{\alpha} \nabla_{y} h}{\sqrt{1+\left|\nabla_{y} h\right|^{2}}}-\frac{\nabla_{y} h\left\langle\nabla_{y} h, \mathcal{Z}^{\alpha} \nabla_{y} h\right\rangle}{\left(1+\left|\nabla_{y} h\right|^{2}\right)^{\frac{3}{2}}}\right)\left(\mathcal{Z}^{\beta} v_{y} \mathcal{Z}^{\gamma} \nabla_{y} h+\frac{\left(\partial_{z} v\right)^{b}}{\partial_{z} \varphi} \mathcal{Z}^{\alpha} h \mathbf{N}\right) d y d \tau \\
= & \sigma \int_{0}^{t} \int_{z=0}\left(\frac{\mathcal{Z}^{\alpha} \nabla_{y} h}{\sqrt{1+\left|\nabla_{y} h\right|^{2}}}-\frac{\nabla_{y} h\left\langle\nabla_{y} h, \mathcal{Z}^{\alpha} \nabla_{y} h\right\rangle}{\left(1+\left|\nabla_{y} h\right|^{2}\right)^{\frac{3}{2}}}\right) \nabla_{y}\left(\mathcal{Z}^{\beta} v_{y} \mathcal{Z}^{\gamma} \nabla_{y} h+\frac{\left(\partial_{z} v\right)^{b}}{\partial_{z} \varphi} \mathcal{Z}^{\alpha} h \mathbf{N}\right) d y d \tau \\
\leq & \Lambda_{\infty}(t) \int_{0}^{t}\|\nabla v\|_{\mathcal{H}^{m-1}}^{2}+\left\|V^{m}\right\|^{2}+|h|_{\mathcal{H}^{m}}^{2}+\sigma\left|\nabla_{y} h\right|_{\mathcal{H}^{m}}^{2} d \tau .
\end{aligned}
$$

Substituting (4.2.7)-(4.2.13), (4.2.22) and (4.2.23) into (4.2.6), one obtains, for $\mathcal{Z}^{\alpha} \neq \partial_{t}^{m}$, that

$$
\begin{aligned}
K_{1} \leq & \Lambda_{0}\left(\sigma\left|\nabla_{y} h_{0}\right|_{\mathcal{H}^{m}}^{2}+\left\|V^{m}(0)\right\|^{2}+\|v(0)\|_{\mathcal{H}^{m-1}}^{2}\right)-\sigma \int_{z=0} \frac{\left|\mathcal{Z}^{\alpha} \nabla_{y} h(t)\right|^{2}}{4\left(1+\left|\nabla_{y} h(t)\right|^{2}\right)^{\frac{3}{2}}} d y \\
& +\frac{1}{16} \int \varrho\left|V^{\alpha}(t)\right|^{2} d \mathcal{V}_{t}+C \delta \int_{0}^{t} \varepsilon^{2}\left\|\nabla^{2} v\right\|_{\mathcal{H}^{m-1}}^{2}+\varepsilon\left\|\nabla V^{m}\right\|_{\mathcal{H}^{m-1}}^{2} d \tau \\
& +C_{\delta} \Lambda_{\infty}(t) \int_{0}^{t} Y_{m}+\|\nabla v\|_{\mathcal{H}^{m-1}}^{2}+\left\|\mathcal{R}_{M}^{\alpha}\right\| Y_{m} d \tau,
\end{aligned}
$$


and for $\mathcal{Z}^{\alpha}=\partial_{t}^{m}$, it holds that

$$
\begin{aligned}
K_{1} \leq & \Lambda_{0}\left[\left|\nabla_{y} h(0)\right|_{\mathcal{H}^{m-1}}^{2}+\sigma\left|\nabla_{y} h_{0}\right|_{\mathcal{H}^{m}}^{2}+\left\|V^{m}(0)\right\|^{2}+\|v(0)\|_{\mathcal{H}^{m-1}}^{2}\right]+\Lambda_{0}\left|\nabla_{y} h\right|_{\mathcal{H}^{m-1}}^{2} \\
& -\sigma \int_{z=0} \frac{\left|\mathcal{Z}^{\alpha} \nabla_{y} h(t)\right|^{2}}{4\left(1+\left|\nabla_{y} h(t)\right|^{2}\right)^{\frac{3}{2}}} d y+\Lambda_{0} \delta \int_{0}^{t} \varepsilon^{2}\left\|\nabla^{2} v\right\|_{\mathcal{H}^{m-1}}^{2}+\varepsilon\left\|\nabla V^{m}\right\|_{\mathcal{H}^{m-1}}^{2} d \tau \\
& +\frac{1}{16} \int \varrho\left|V^{\alpha}(t)\right|^{2} d \mathcal{V}_{t}+C_{\delta} \Lambda_{\infty}(t) \int_{0}^{t} Y_{m}+\|\nabla v\|_{\mathcal{H}^{m-1}}^{2}+\left\|\mathcal{R}_{M}^{\alpha}\right\| Y_{m} d \tau,
\end{aligned}
$$

where the remaining term $\left\|\mathcal{R}_{M}^{\alpha}\right\|$ in (4.2.24) and (4.2.25) will be estimated later.

It follows from (3.31), (3.32) and (2.5) that

$$
\begin{aligned}
K_{2}= & \int_{0}^{t} \int_{z=0} \partial_{z}^{\varphi} p \mathcal{Z}^{\alpha} h\left(\partial_{t} \mathcal{Z}^{\alpha} h-v^{b} \cdot \mathcal{Z}^{\alpha} \mathbf{N}-\mathcal{C}^{\alpha}(h)\right) d y d \tau \\
= & \frac{1}{2} \int_{z=0} \partial_{z}^{\varphi} p(t)\left|\mathcal{Z}^{\alpha} h(t)\right|^{2} d y-\frac{1}{2} \int_{z=0} \partial_{z}^{\varphi} p(0)\left|\mathcal{Z}^{\alpha} h(0)\right|^{2} d y \\
& -\frac{1}{2} \int_{0}^{t} \int_{z=0} \partial_{t} \partial_{z}^{\varphi} p\left|\mathcal{Z}^{\alpha} h\right|^{2} d y d \tau-\int_{0}^{t} \int_{z=0} \partial_{z}^{\varphi} p \mathcal{Z}^{\alpha} h\left(v^{b} \cdot \mathcal{Z}^{\alpha} \mathbf{N}+\mathcal{C}^{\alpha}(h)\right) d y d \tau \\
\leq & \frac{1}{2} \int_{z=0} \partial_{z}^{\varphi} p(t)\left|\mathcal{Z}^{\alpha} h(t)\right|^{2} d y-\frac{1}{2} \int_{z=0} \partial_{z}^{\varphi} p(0)\left|\mathcal{Z}^{\alpha} h(0)\right|^{2} d y+\Lambda_{\infty}(t) \int_{0}^{t}|h|_{\mathcal{H}^{m}}^{2} d \tau \\
& -\int_{0}^{t} \int_{z=0} \partial_{z}^{\varphi} p v^{b} \cdot \nabla_{y}\left(\frac{\left|\mathcal{Z}^{\alpha} h\right|^{2}}{2}\right)+\partial_{z}^{\varphi} p \mathcal{Z}^{\alpha} h \mathcal{C}^{\alpha}(h) d y d \tau \\
\leq & \frac{1}{2} \int_{z=0} \partial_{z}^{\varphi} p(t)\left|\mathcal{Z}^{\alpha} h(t)\right|^{2} d y-\frac{1}{2} \int_{z=0} \partial_{z}^{\varphi} p(0)\left|\mathcal{Z}^{\alpha} h(0)\right|^{2} d y \\
& +\Lambda_{\infty}(t) \int_{0}^{t}\|\nabla v\|_{\mathcal{H}^{m-1}}^{2}+|h|_{\mathcal{H}^{m}}^{2}+\|v\|_{\mathcal{H}^{m-1}}^{2} d \tau
\end{aligned}
$$

Since the boundary condition (1.33) yields immediately that

$$
p-p_{e}=\left(2 \mu \varepsilon S^{\varphi} v+\lambda \varepsilon \operatorname{div}^{\varphi} v\right) \mathbf{n} \cdot \mathbf{n}-\sigma \nabla_{y} \cdot \frac{\nabla_{y} h}{\sqrt{1+\left|\nabla_{y} h\right|^{2}}} .
$$

This, together with Lemma 2.3 and trace theorem, implies that

$$
\begin{aligned}
K_{3} & \leq \Lambda_{\infty}(t) \varepsilon\left(\int_{0}^{t}\left|\mathcal{Z}^{\alpha} \mathbf{N}\right|_{-\frac{1}{2}}^{2}\right)^{\frac{1}{2}}\left(\int_{0}^{t}\left|\left(V^{\alpha}\right)^{b}\right|_{\frac{1}{2}}^{2} d \tau\right)^{\frac{1}{2}} \\
& \leq \delta \varepsilon \int_{0}^{t}\left\|\nabla V^{m}\right\|^{2} d \tau+C_{\delta} \Lambda_{\infty}(t) \int_{0}^{t} \varepsilon\left|\mathcal{Z}^{m} h\right|_{\frac{1}{2}}^{2} d \tau .
\end{aligned}
$$

Direct calculations yield immediately that

$$
\begin{aligned}
K_{4} & \leq \Lambda\left(\frac{1}{c_{0}},\|v\|_{\mathcal{W}^{2, \infty}, t}+\|\nabla v\|_{\mathcal{W}^{1, \infty}, t}\right) \varepsilon^{\frac{1}{2}}\left\|\partial_{z z} v\right\|_{\infty, t} \varepsilon^{\frac{1}{2}} \int_{0}^{t}|h|_{\mathcal{H}^{m}}\left|V^{\alpha}\right| d \tau \\
& \leq \delta \varepsilon \int_{0}^{t}\left\|\nabla V^{m}\right\|^{2} d \tau+C_{\delta} \Lambda_{\infty}(t) \int_{0}^{t}\left(|h|_{\mathcal{H}^{m}}^{2}+\left\|V^{m}\right\|^{2}\right) d \tau .
\end{aligned}
$$

It follows from (3.27) that

$$
\begin{aligned}
& K_{5} \leq \int_{0}^{t}\left|\mathcal{C}^{\alpha}(B)\right|_{L^{2}}\left|V^{\alpha}\right|_{L^{2}} d \tau \leq\left(\int_{0}^{t}\left|\mathcal{C}^{\alpha}(\mathcal{B})\right|_{L^{2}}^{2} d \tau\right)^{\frac{1}{2}}\left(\int_{0}^{t}\left\|V^{\alpha}\right\|\left\|\nabla V^{\alpha}\right\|\right)^{\frac{1}{2}} \\
& \leq \Lambda_{\infty}(t)\left\{\varepsilon^{2} \int_{0}^{t}\left|(\nabla v)^{b}\right|_{\mathcal{H}^{m-1}}^{2}+\left|\nabla_{y} h\right|_{\mathcal{H}^{m-1}}^{2} d \tau\right\}^{\frac{1}{2}}\left\{\int_{0}^{t}\left\|\nabla V^{\alpha}\right\|\left\|V^{\alpha}\right\|+\left\|V^{\alpha}\right\|^{2}\right\}^{\frac{1}{2}} \\
& \leq \delta \varepsilon \int_{0}^{t}\left\|\nabla V^{m}\right\|^{2} d \tau+\delta \varepsilon^{2} \int_{0}^{t}\left\|\nabla^{2} v\right\|_{\mathcal{H}^{m-1}}^{2} d \tau+C_{\delta} \Lambda_{\infty}(t) \int_{0}^{t} Y_{m}+\|\nabla v\|_{\mathcal{H}^{m-1}}^{2} d \tau .
\end{aligned}
$$


Therefore, from (4.2.24)- 4.2.29), one obtains the boundary estimate for $\mathcal{Z}^{\alpha} \neq \partial_{t}^{m}$ that

$$
\begin{aligned}
\int_{0}^{t} \mathcal{B} d \tau \leq & \Lambda_{0}\left[\left|h_{0}\right|_{\mathcal{H}^{m}}^{2}+\sigma\left|\nabla_{y} h_{0}\right|_{\mathcal{H}^{m}}^{2}+\left\|V^{m}(0)\right\|^{2}+\|v(0)\|_{\mathcal{H}^{m-1}}^{2}\right]+\frac{1}{2} \int_{z=0} \partial_{z}^{\varphi} p(t)\left|\mathcal{Z}^{\alpha} h(t)\right|^{2} d y \\
& -\sigma \int_{z=0} \frac{\left|\nabla_{y} \mathcal{Z}^{\alpha} h(t)\right|^{2}}{4\left(1+\left|\nabla_{y} h(t)\right|^{2}\right)^{\frac{3}{2}}} d y+C \delta \int_{0}^{t} \varepsilon^{2}\left\|\nabla^{2} v\right\|_{\mathcal{H}^{m-1}}^{2}+\varepsilon\left\|\nabla V^{m}\right\|^{2} d \tau \\
& +C_{\delta} \Lambda_{\infty}(t) \int_{0}^{t} Y_{m}+\|\nabla v\|_{\mathcal{H}^{m-1}}^{2}+\left\|\mathcal{R}_{M}^{\alpha}\right\| Y_{m} d \tau+\frac{1}{16} \int \varrho\left|V^{\alpha}(t)\right|^{2} d \mathcal{V}_{t}
\end{aligned}
$$

and for $\mathcal{Z}^{\alpha}=\partial_{t}^{m}$, it holds that

$$
\begin{aligned}
\int_{0}^{t} \mathcal{B} d \tau \leq & \Lambda_{0}\left[\left|h_{0}\right|_{\mathcal{H}^{m}}^{2}+\sigma\left|\nabla_{y} h_{0}\right|_{\mathcal{H}^{m}}^{2}+\left\|V^{m}(0)\right\|^{2}+\|v(0)\|_{\mathcal{H}^{m-1}}^{2}\right]+\Lambda_{0}\left|\nabla_{y} h\right|_{\mathcal{H}^{m-1}}^{2} \\
& +\frac{1}{16} \int \varrho\left|V^{\alpha}(t)\right|^{2} d \mathcal{V}_{t}+\frac{1}{2} \int_{z=0} \partial_{z}^{\varphi} p(t)\left|\mathcal{Z}^{\alpha} h(t)\right|^{2} d y-\sigma \int_{z=0} \frac{\left|\nabla_{y} \mathcal{Z}^{\alpha} h(t)\right|^{2}}{4\left(1+\left|\nabla_{y} h(t)\right|^{2}\right)^{\frac{3}{2}}} d y \\
& +C \delta \int_{0}^{t} \varepsilon^{2}\left\|\nabla^{2} v\right\|_{\mathcal{H}^{m-1}}^{2}+\varepsilon\left\|\nabla V^{m}\right\|^{2} d \tau+C_{\delta} \Lambda_{\infty}(t) \int_{0}^{t} Y_{m}+\|\nabla v\|_{\mathcal{H}^{m-1}}^{2}+\left\|\mathcal{R}_{M}^{\alpha}\right\| Y_{m} d \tau
\end{aligned}
$$

Using (3.5) and integration by parts, one obtains that

$$
\begin{aligned}
& \int_{0}^{t} J d \tau=\int_{0}^{t} \int_{\mathcal{S}} Q^{\alpha}\left(-\frac{1}{\gamma p} \partial_{t}^{\varphi} Q^{\alpha}-\frac{1}{\gamma p} v \cdot \nabla^{\varphi} Q^{\alpha}+\mathcal{R}_{d}^{\alpha}\right) d \mathcal{V}_{\tau} d \tau \\
& =-\int_{\mathcal{S}} \frac{\left|Q^{\alpha}(t)\right|^{2}}{2 \gamma p(t)} d \mathcal{V}_{t}+\int_{\mathcal{S}} \frac{\left|Q^{\alpha}(0)\right|^{2}}{2 \gamma p(0)} d \mathcal{V}_{0} \\
& \quad+\int_{0}^{t} \int_{\mathcal{S}}\left(\partial_{t}\left(\frac{1}{2 \gamma p}\right)+d_{i v}^{\varphi}\left(\frac{v}{2 \gamma p}\right)\right)\left|Q^{\alpha}\right|^{2} d \mathcal{V}_{\tau}+\int_{0}^{t} \int_{\mathcal{S}} Q^{\alpha} \mathcal{R}_{d}^{\alpha} d \mathcal{V}_{\tau} \\
& \leq-\int_{\mathcal{S}} \frac{\left|Q^{\alpha}(t)\right|^{2}}{2 \gamma p(t)} d \mathcal{V}_{t}+\int_{\mathcal{S}} \frac{\left|Q^{\alpha}(0)\right|^{2}}{2 \gamma p(0)} d \mathcal{V}_{0}+\Lambda_{\infty}(t) \int_{0}^{t}\left\|Q^{m}(t)\right\|^{2} d \tau+\int_{0}^{t} \int_{\mathcal{S}} Q^{\alpha} \mathcal{R}_{d}^{\alpha} d \mathcal{V}_{\tau} d \tau,
\end{aligned}
$$

where

$\mathcal{R}_{d}^{\alpha}=\mathcal{Z}^{\alpha} \eta\left(\partial_{z}^{\varphi}\left(\frac{1}{\gamma p}\right) \partial_{t}^{\varphi} p+\partial_{z}^{\varphi}\left(\frac{v}{\gamma p}\right) \cdot \nabla^{\varphi} p\right)-\mathcal{C}^{\alpha}(d)-\frac{1}{\gamma p} \mathcal{C}_{0}^{\alpha}(p)-\frac{v}{\gamma p} \cdot \mathcal{C}^{\alpha}(p)-\left[\mathcal{Z}^{\alpha}, \frac{1}{\gamma p}\right] \partial_{t}^{\varphi} p-\left[\mathcal{Z}^{\alpha}, \frac{v}{\gamma p}\right] \cdot \nabla^{\varphi} p$.

Furthermore, it is easy to obtain that

$$
\int_{0}^{t} \int_{\mathcal{S}} Q^{\alpha} \mathcal{Z}^{\alpha} \eta\left(\partial_{z}^{\varphi}\left(\frac{1}{\gamma p}\right) \partial_{t}^{\varphi} p+\partial_{z}^{\varphi}\left(\frac{v}{\gamma p}\right) \cdot \nabla^{\varphi} p\right) d \mathcal{V}_{\tau} d \tau \leq \Lambda_{\infty}(t) \int_{0}^{t}\left\|Q^{m}(t)\right\|^{2}+|h|_{\mathcal{H}^{m}}^{2} d \tau
$$

It follows from (3.7)-3.8 that

$$
\begin{aligned}
& \int_{0}^{t} \int_{\mathcal{S}} Q^{\alpha}\left(-\mathcal{C}^{\alpha}(d)-\frac{1}{\gamma p} \mathcal{C}_{0}^{\alpha}(p)-\frac{v}{\gamma p} \cdot \mathcal{C}^{\alpha}(p)\right) d \mathcal{V}_{\tau} d \tau \\
& \leq \Lambda\left(\frac{1}{c_{0}},\|v\|_{\infty, t}\right)\left(\int_{0}^{t}\left\|Q^{\alpha}\right\|^{2} d \tau\right)^{\frac{1}{2}}\left(\int_{0}^{t}\left(\left\|\mathcal{C}^{\alpha}(d)\right\|+\left\|\mathcal{C}_{0}^{\alpha}(p)\right\|+C^{\alpha}(p) \|\right)^{2} d \tau\right)^{\frac{1}{2}} \\
& \leq \delta \int_{0}^{t}\|\nabla p\|_{\mathcal{H}^{m-1}}^{2} d \tau+C_{\delta} \Lambda_{\infty}(t) \int_{0}^{t}\|\nabla v\|_{\mathcal{H}^{m-1}}^{2}+\left\|Q^{m}(t)\right\|^{2}+|h|_{\mathcal{H}^{m}}^{2} d \tau
\end{aligned}
$$

Using (2.2), (4.0.5) and (4.0.6), one gets that

$$
\begin{aligned}
& \int_{0}^{t} \int_{\mathcal{S}} Q^{\alpha}\left(-\left[\mathcal{Z}^{\alpha}, \frac{1}{\gamma p}\right] \partial_{t}^{\varphi} p-\left[\mathcal{Z}^{\alpha}, \frac{v}{\gamma p}\right] \cdot \nabla^{\varphi} p\right) d \mathcal{V}_{\tau} d \tau \\
& \leq \int_{0}^{t}\left\|Q^{\alpha}\right\|\left(\left\|\left[\mathcal{Z}^{\alpha}, \frac{1}{\gamma p}\right] \partial_{t}^{\varphi} p\right\|+\left\|\left[\mathcal{Z}^{\alpha}, \frac{v}{\gamma p}\right] \cdot \nabla^{\varphi} p\right\|\right) d \tau \\
& \leq \Lambda_{\infty}(t)\left(\int_{0}^{t}\left\|Q^{\alpha}\right\|^{2} d \tau\right)^{\frac{1}{2}}\left(\int_{0}^{t}\left(\|p\|_{\mathcal{H}^{m}}^{2}+\|\nabla p\|_{\mathcal{H}^{m-1}}^{2}+\|v\|_{\mathcal{H}^{m}}^{2}+|h|_{\mathcal{H}^{m}}^{2} d \tau\right)^{\frac{1}{2}}\right.
\end{aligned}
$$




$$
\begin{aligned}
& \leq \delta \int_{0}^{t}\|\nabla p\|_{\mathcal{H}^{m-1}}^{2} d \tau+C_{\delta} \Lambda_{\infty}(t) \int_{0}^{t}\|v\|_{\mathcal{H}^{m}}^{2}+\|p\|_{\mathcal{H}^{m}}^{2}+\left\|Q^{\alpha}\right\|^{2}+|h|_{\mathcal{H}^{m}}^{2} d \tau \\
& \leq \delta \int_{0}^{t}\|\nabla p\|_{\mathcal{H}^{m-1}}^{2} d \tau+C_{\delta} \Lambda_{\infty}(t) \int_{0}^{t}\left\|V^{m}(t)\right\|^{2}+\left\|Q^{m}(t)\right\|^{2}+|h|_{\mathcal{H}^{m}}^{2} d \tau
\end{aligned}
$$

Combining (4.2.32)-4.2.35) leads to that

$$
\begin{aligned}
\int_{0}^{t} J d \tau \leq & -\int_{\mathcal{S}} \frac{\left|Q^{\alpha}(t)\right|^{2}}{2 \gamma p(t)} d \mathcal{V}_{t}+\int_{\mathcal{S}} \frac{\left|Q^{\alpha}(0)\right|^{2}}{2 \gamma p(0)} d \mathcal{V}_{t}+\delta \int_{0}^{t}\|\nabla p\|_{\mathcal{H}^{m-1}}^{2} d \tau \\
& +C_{\delta} \Lambda_{\infty}(t) \int_{0}^{t}\|\nabla v\|_{\mathcal{H}^{m-1}}^{2}+\left\|V^{m}(t)\right\|^{2}+\left\|Q^{m}(t)\right\|^{2}+|h|_{\mathcal{H}^{m}}^{2} d \tau
\end{aligned}
$$

Now we estimate the terms involve $\mathcal{R}_{M}^{\alpha}$. Using (3.6)-(3.10), it is easy to obtain that

$$
\int_{0}^{t}\left\|\mathcal{R}_{M}^{\alpha}\right\|^{2} d \tau \leq \Lambda_{\infty}(t) \int_{0}^{t} \varepsilon^{2}\left\|\nabla^{2} v\right\|_{\mathcal{H}^{m-1}}^{2}+Y_{m}+\varepsilon\left|\mathcal{Z}^{m} h\right|_{\frac{1}{2}}^{2} d \tau
$$

Therefore, using holder inequality, one gets that

$$
\begin{aligned}
& \left|\int_{0}^{t} \int_{\mathcal{S}} \mathcal{R}_{M}^{\alpha} V^{\alpha} d \mathcal{V}_{\tau} d \tau\right| \leq \int_{0}^{t}\left\|\mathcal{R}_{M}^{\alpha}\right\|\left\|V^{\alpha}\right\| d \tau \\
& \leq \delta \int_{0}^{t} \varepsilon^{2}\left\|\nabla^{2} v\right\|_{\mathcal{H}^{m-1}}^{2} d \tau+C_{\delta} \Lambda_{\infty}(t) \int_{0}^{t} Y_{m}+\|\nabla v\|_{\mathcal{H}^{m-1}}^{2}+\varepsilon\left|\mathcal{Z}^{m} h\right|_{\frac{1}{2}}^{2} d \tau,
\end{aligned}
$$

and

$$
\int_{0}^{t}\left\|\mathcal{R}_{M}^{\alpha}\right\| Y_{m} d \tau \leq \delta \int_{0}^{t} \varepsilon^{2}\left\|\nabla^{2} v\right\|_{\mathcal{H}^{m-1}}^{2} d \tau+C_{\delta} \Lambda_{\infty}(t) \int_{0}^{t} Y_{m}+\|\nabla v\|_{\mathcal{H}^{m-1}}^{2}+\varepsilon\left|\mathcal{Z}^{m} h\right|_{\frac{1}{2}}^{2} d \tau
$$

Combining (4.2.4), (4.2.30), (4.2.36), (4.2.37) and (4.2.38), we obtain, for $\mathcal{Z}^{\alpha} \neq \partial_{t}^{m}$, that

$$
\begin{aligned}
& \int_{\mathcal{S}} \frac{\varrho}{4}\left|V^{\alpha}(t)\right|+\frac{\left|Q^{\alpha}(t)\right|^{2}}{2 \gamma p(t)} d \mathcal{V}_{t}+\frac{1}{2} \int_{z=0}-\partial_{z}^{\varphi} p(t)\left|\mathcal{Z}^{\alpha} h(t)\right|^{2}+\sigma \frac{\left|\nabla_{y} \mathcal{Z}^{\alpha} h(t)\right|^{2}}{\left(1+\left|\nabla_{y} h(t)\right|^{2}\right)^{\frac{3}{2}}} d y \\
& \quad+\int_{0}^{t} \int_{\mathcal{S}} 2 \mu \varepsilon\left|S^{\varphi} V^{\alpha}\right|^{2}+\lambda \varepsilon\left|d i v^{\varphi} V^{\alpha}\right|^{2} d \mathcal{V}_{\tau} d \tau \\
& \leq \Lambda_{0}\left[\left|\left(h, \sqrt{\sigma} \nabla_{y} h\right)(0)\right|_{\mathcal{H}^{m}}^{2}+\left\|\left(V^{m}, Q^{m}\right)(0)\right\|^{2}+\|v(0)\|_{\mathcal{H}^{m}}^{2}\right]+\delta \int_{0}^{t}\|\nabla p\|_{\mathcal{H}^{m-1}}^{2} d \tau \\
& \quad+\Lambda_{0} \delta \int_{0}^{t} \varepsilon\left\|\nabla V^{m}\right\|^{2}+\varepsilon^{2}\left\|\nabla^{2} v\right\|_{\mathcal{H}^{m-1}}^{2} d \tau+\Lambda_{\infty}(t) \int_{0}^{t} \Lambda\left(Y_{m}\right)+\|\nabla v\|_{\mathcal{H}^{m-1}}^{2}+\varepsilon\left|\mathcal{Z}^{m} h\right|_{\frac{1}{2}}^{2} d \tau .
\end{aligned}
$$

Therefore, summing over $\alpha$, using the a priori assumptions (4.0.4) on the Taylor sign condition and Lemma 2.10, and taking $\delta$ suitably small, one proves 4.2.1) for the case $\mathcal{Z}^{\alpha} \neq \partial_{t}^{m}$.

Collecting (4.2.4), 4.2.31), 44.2.36), 4.2.37), 4.2.38), and using 4.2.1 for the case of $\mathcal{Z}^{\alpha} \neq \partial_{t}^{m}$ whose proof has already been closed in (4.2.39), one yields immediately (4.2.1) for the case $\mathcal{Z}^{\alpha}=\partial_{t}^{m}$. Therefore, the proof of Lemma 4.4 is completed.

\subsection{Estimates for $\nabla^{\varphi} p$ and $d i v^{\varphi} v$}

To deal with the compressibility, one needs to obtain some uniform estimates for the pressure and the divergence of the velocity field. For incompressible flows, the divergence of the velocity field is zero and the pressure plays a role of Lagrangian multiplier so that the pressure can be estimated by elliptic regularities, see 32 . While, the pressure $p(\varrho)=\varrho^{\gamma}$, shown in (1.31) ${ }_{2}$ for compressible flows, satisfies a transport equation due to(1.31) 1 so that it can be estimated by energy method. However, it seems that one can only expect uniform estimates of $m-2$ order conormal derivatives of $\nabla p$, which is one order lower than the one in incompressible flows in 32 , since the divergence of the velocity field is not free but depends on the regularity of the free surfaces in compressible flows.

Precisely, one can obtain the following estimates for $\nabla^{\varphi} p$ and $\operatorname{div}^{\varphi} v$. 
Proposition 4.5 For any $t \in\left[0, T^{\varepsilon}\right]$ and $m \geq 2$, it holds that

$$
\begin{aligned}
& \|\nabla v(t)\|_{\mathcal{H}^{m-2}}^{2}+\varepsilon\left\|\left(d i v^{\varphi} v, \nabla^{\varphi} p\right)(t)\right\|_{\mathcal{H}^{m-1}}^{2}+\varepsilon^{2} \int_{0}^{t}\left\|\nabla^{\varphi} \mathcal{Z}^{m-1} d i v^{\varphi} v\right\|^{2} d \tau \\
& \leq\left\|\nabla v_{0}\right\|_{\mathcal{H}^{m-2}}^{2}+\Lambda_{0} \varepsilon\left\|\left(d i v^{\varphi} v_{0}, \nabla^{\varphi} p_{0}\right)\right\|_{\mathcal{H}^{m-1}}^{2}+\left(\delta_{1}+\delta \Lambda_{0}\right) \varepsilon^{2} \int_{0}^{t}\left\|\nabla^{2} v\right\|_{\mathcal{H}^{m-1}}^{2} d \tau \\
& \quad+\frac{\Lambda_{0}}{\delta_{1}} \varepsilon \int_{0}^{t}\left\|\nabla V^{m}\right\|^{2} d \tau+C_{\delta} \Lambda_{\infty}(t)\left(1+\left\|\nabla d i v^{\varphi} v\right\|_{\infty, t}^{2}\right) \int_{0}^{t} \Lambda\left(Y_{m}\right)+\|\nabla v\|_{\mathcal{H}^{m-1}}^{2}+\varepsilon\left|\mathcal{Z}^{m} h\right|_{\frac{1}{2}}^{2} d \tau,
\end{aligned}
$$

and

$$
\|\nabla p(t)\|_{\mathcal{H}^{m-2}}^{2}+\int_{0}^{t}\|\nabla p\|_{\mathcal{H}^{m-1}}^{2} d \tau \leq \Lambda_{0} \varepsilon^{2} \int_{0}^{t}\left\|\nabla^{2} v\right\|_{\mathcal{H}^{m-1}}^{2} d \tau+\Lambda_{\infty}(t) \int_{0}^{t} \Lambda\left(Y_{m}\right) d \tau,
$$

where $\delta_{1}>0$ and $\delta>0$ are small constants which will be chosen later.

It should be noted that the estimate of $\varepsilon^{2} \int_{0}^{t}\left\|\nabla^{\varphi} \mathcal{Z}^{m-1} \operatorname{div}^{\varphi} v\right\|^{2} d \tau$ is the key to control $\varepsilon^{2} \int_{0}^{t}\left\|\nabla^{2} v\right\|_{\mathcal{H}^{m-1}}^{2} d \tau$. This is not necessary for incompressible case, since $\varepsilon^{2} \int_{0}^{t}\left\|\nabla^{2} v\right\|_{\mathcal{H}^{m-1}}^{2} d \tau$ does not appear due to (1.62).

The proof of this proposition is a consequence of the following lemmas. Note that

$$
\left\{\begin{array}{l}
\|\nabla v(t)\|_{\mathcal{H}^{m-2}}^{2} \leq\left\|\nabla v_{0}\right\|_{\mathcal{H}^{m-2}}^{2}+\int_{0}^{t}\left\|\partial_{t} \nabla v\right\|_{\mathcal{H}^{m-2}}^{2} d \tau \leq\left\|\nabla v_{0}\right\|_{\mathcal{H}^{m-2}}^{2}+\int_{0}^{t}\|\nabla v\|_{\mathcal{H}^{m-1}}^{2} d \tau, \\
\|\nabla p(t)\|_{\mathcal{H}^{m-2}}^{2} \leq\left\|\nabla p_{0}\right\|_{\mathcal{H}^{m-2}}^{2}+\int_{0}^{t}\left\|\partial_{t} \nabla p\right\|_{\mathcal{H}^{m-2}}^{2} d \tau \leq\left\|\nabla p_{0}\right\|_{\mathcal{H}^{m-2}}^{2}+\int_{0}^{t}\|\nabla p\|_{\mathcal{H}^{m-1}}^{2} d \tau
\end{array}\right.
$$

We will estimate $\varepsilon\left\|\left(\nabla^{\varphi} p, \operatorname{div}^{\varphi} v\right)\right\|_{\mathcal{H}^{m-1}}, \varepsilon^{2} \int_{0}^{t}\left\|\nabla^{\varphi} \mathcal{Z}^{m-1} \operatorname{div}^{\varphi} v\right\|^{2} d \tau$ and $\int_{0}^{t}\|\nabla p\|_{\mathcal{H}^{m-1}}^{2} d \tau$ in the remaining part of this subsection. The estimate of $\int_{0}^{t}\|\nabla v\|_{\mathcal{H}^{m-1}}^{2} d \tau$ will be left to later subsections.

The first lemma gives the equations satisfied by $\mathcal{Z}^{\alpha} v$ and $\mathcal{Z}^{\alpha} \nabla^{\varphi} p$.

Lemma 4.6 For $|\alpha| \leq m$, the equations for $\mathcal{Z}^{\alpha} \nabla^{\varphi} p$ and $\mathcal{Z}^{\alpha}$ div $v$ read as

$$
\left\{\begin{array}{l}
\mathcal{Z}^{\alpha} d i v^{\varphi} v+\frac{1}{\gamma p}\left(\partial_{t}^{\varphi}+v \cdot \nabla^{\varphi}\right) \mathcal{Z}^{\alpha} p=\mathcal{C}_{T}^{\alpha}(p), \\
\varrho\left(\partial_{t}^{\varphi}+v \cdot \nabla^{\varphi}\right) \mathcal{Z}^{\alpha} v+\mathcal{Z}^{\alpha} \nabla^{\varphi} p-\mathcal{Z}^{\alpha}\left(2 \mu \varepsilon d i v^{\varphi} S^{\varphi} v+\lambda \varepsilon \nabla^{\varphi} \operatorname{div}^{\varphi} v\right)=\mathcal{C}_{M}^{\alpha}(p),
\end{array}\right.
$$

where the commutators are given by

$$
\begin{aligned}
& \mathcal{C}_{T}^{\alpha}(p):=-\frac{1}{\gamma p}\left(\left[\mathcal{Z}^{\alpha}, v_{y}\right] \nabla_{y} p+\left[\mathcal{Z}^{\alpha}, V_{z}\right] \partial_{z} p+V_{z}\left[\mathcal{Z}^{\alpha}, \partial_{z}\right] p\right)-\left[\mathcal{Z}^{\alpha}, \frac{1}{\gamma p}\right]\left(\partial_{t}^{\varphi} p+v \cdot \nabla^{\varphi} p\right), \\
& \mathcal{C}_{M}^{\alpha}(p):=-\varrho\left(\left[\mathcal{Z}^{\alpha}, v_{y}\right] \nabla_{y} v+\left[\mathcal{Z}^{\alpha}, V_{z}\right] \partial_{z} v+V_{z}\left[\mathcal{Z}^{\alpha}, \partial_{z}\right] v\right)-\left[\mathcal{Z}^{\alpha}, \varrho\right]\left(\partial_{t}^{\varphi} v+v \cdot \nabla^{\varphi} v\right) .
\end{aligned}
$$

Moreover, these commutators vanish when $|\alpha|=0$.

Proof. The mass equation (1.31) 1 yields that

$$
\operatorname{div}^{\varphi} v+\frac{1}{\gamma p}\left(\partial_{t}^{\varphi} p+v \cdot \nabla^{\varphi} p\right)=0 .
$$

Applying $\mathcal{Z}^{\alpha}$ to (4.3.5) and (1.31) 2 shows the lemma by trivial calculations.

Lemma 4.7 For any $t \in\left[0, T^{\varepsilon}\right], m \geq 1$, it holds that

$$
\begin{gathered}
\varepsilon\left\|\left(d i v^{\varphi} v, \nabla^{\varphi} p\right)(t)\right\|_{\mathcal{H}^{m-1}}^{2}+\varepsilon^{2} \int_{0}^{t}\left\|\nabla^{\varphi} \mathcal{Z}^{m-1} d i v^{\varphi} v\right\|^{2} d \tau \\
\leq \Lambda_{0} \varepsilon\left\|\left(d i v^{\varphi_{0}} v_{0}, \nabla^{\varphi_{0}} p_{0}\right)\right\|_{\mathcal{H}^{m-1}}^{2}+\delta_{1} \int_{0}^{t} \varepsilon^{2}\left\|\nabla^{2} v\right\|_{\mathcal{H}^{m-1}}^{2}+\delta \int_{0}^{t}\|\nabla p\|_{\mathcal{H}^{m-1}}^{2} d \tau \\
+\frac{\Lambda_{0}}{\delta_{1}} \varepsilon \int_{0}^{t}\left\|\nabla V^{m}\right\|^{2} d \tau+C_{\delta} \Lambda_{\infty}(t) \varepsilon^{2} \int_{0}^{t}\left\|\nabla^{2} v\right\|_{\mathcal{H}^{m-2}}^{2} d \tau \\
+C_{\delta_{1}} \Lambda_{\infty}(t)\left(1+\left\|\nabla d i v^{\varphi} v\right\|_{L_{t, x}^{\infty}}^{2}\right) \int_{0}^{t} \Lambda\left(Y_{m}(\tau)\right)+\varepsilon\left|\mathcal{Z}^{m} h\right|_{\frac{1}{2}}^{2} d \tau
\end{gathered}
$$

where $\delta$ and $\delta_{1}$ will be chosen later. 
Proof. Multiplying (4.3.4) ${ }_{2}$ by $\varepsilon \nabla^{\varphi} \mathcal{Z}^{\alpha} \operatorname{div}^{\varphi} v$ and integrating over $\mathcal{S} \times[0, t]$ lead to that

$$
\begin{aligned}
& \varepsilon \int_{0}^{t} \int_{\mathcal{S}} \varrho\left(\partial_{t}^{\varphi}+v \cdot \nabla^{\varphi}\right) \mathcal{Z}^{\alpha} v \cdot \nabla^{\varphi} \mathcal{Z}^{\alpha} \operatorname{div}^{\varphi} v d \mathcal{V}_{\tau} d \tau+\varepsilon \int_{0}^{t} \int_{\mathcal{S}} \mathcal{Z}^{\alpha} \nabla^{\varphi} p \cdot \nabla^{\varphi} \mathcal{Z}^{\alpha} \operatorname{div}^{\varphi} v d \mathcal{V}_{\tau} d \tau \\
& =(2 \mu+\lambda) \varepsilon^{2} \int_{0}^{t} \int_{\mathcal{S}} \mathcal{Z}^{\alpha} \nabla^{\varphi} \operatorname{div}^{\varphi} v \cdot \nabla^{\varphi} \mathcal{Z}^{\alpha} \operatorname{div}^{\varphi} v d \mathcal{V}_{\tau} d \tau \\
& \quad-\mu \varepsilon^{2} \int_{0}^{t} \int_{\mathcal{S}} \mathcal{Z}^{\alpha}\left(\nabla^{\varphi} \times\left(\nabla^{\varphi} \times v\right)\right) \cdot \nabla^{\varphi} \mathcal{Z}^{\alpha} \operatorname{div}^{\varphi} v d \mathcal{V}_{\tau} d \tau \\
& \quad+\varepsilon \int_{0}^{t} \int_{\mathcal{S}} \mathcal{C}_{M}^{\alpha}(p) \cdot \nabla^{\varphi} \mathcal{Z}^{\alpha} \operatorname{div}^{\varphi} v d \mathcal{V}_{\tau} d \tau=\sum_{i=1}^{3} J_{i}
\end{aligned}
$$

Integration by parts shows that

$$
\begin{aligned}
& \varepsilon \int_{0}^{t} \int_{\mathcal{S}} \varrho\left(\partial_{t}^{\varphi}+v \cdot \nabla^{\varphi}\right) \mathcal{Z}^{\alpha} v \cdot \nabla^{\varphi} \mathcal{Z}^{\alpha} \operatorname{div}^{\varphi} v d \mathcal{V}_{\tau} d \tau \\
& =-\varepsilon \int_{0}^{t} \int_{\mathcal{S}} \varrho\left(\partial_{t}^{\varphi}+v \cdot \nabla^{\varphi}\right) \operatorname{div}^{\varphi} \mathcal{Z}^{\alpha} v \mathcal{Z}^{\alpha} \operatorname{div}^{\varphi} v d \mathcal{V}_{\tau} d \tau \\
& \quad-\varepsilon \int_{0}^{t} \int_{\mathcal{S}}\left(\nabla^{\varphi} \varrho\left(\partial_{t}+v_{y} \cdot \nabla_{y}+V_{z} \partial_{z}\right) \mathcal{Z}^{\alpha} v+\varrho\left(\nabla^{\varphi} v\right)^{t} \nabla^{\varphi} \mathcal{Z}^{\alpha} v\right) \mathcal{Z}^{\alpha} \operatorname{div}^{\varphi} v d \mathcal{V}_{\tau} d \tau \\
& \quad+\varepsilon \int_{0}^{t} \int_{z=0} \varrho\left(\partial_{t}+v_{y} \cdot \nabla_{y}+V_{z} \partial_{z}\right) \mathcal{Z}^{\alpha} v \cdot \mathbf{N} \mathcal{Z}^{\alpha} \operatorname{div}^{\varphi} v d \mathcal{V}_{\tau} d \tau=\sum_{1}^{3} K_{i} .
\end{aligned}
$$

Note that

$$
\mathcal{Z}\left[\partial_{i}^{\varphi}, \mathcal{Z}^{\alpha}\right] f=-\frac{\partial_{i} \varphi}{\partial_{z} \varphi}\left[\mathcal{Z}, \partial_{z}\right] \mathcal{Z}^{\alpha} f-\mathcal{Z}\left(\frac{\partial_{i} \varphi}{\partial_{z} \varphi}\right) \partial_{z} \mathcal{Z}^{\alpha} f+\left[\partial_{i}^{\varphi}, \mathcal{Z} \mathcal{Z}^{\alpha}\right] f, \quad\left[Z_{3}, \partial_{z}\right]=-\frac{1}{(1-z)^{2}} \partial_{z}
$$

It follows from (2.1), (2.2) and (2.13) that

$$
\begin{aligned}
K_{1}= & -\frac{1}{2} \varepsilon \int_{\mathcal{S}} \varrho\left|\mathcal{Z}^{\alpha} \operatorname{div}^{\varphi} v(t)\right|^{2} d \mathcal{V}_{t}+\frac{1}{2} \varepsilon \int_{\mathcal{S}} \varrho_{0}\left|\mathcal{Z}^{\alpha} \operatorname{div}^{\varphi_{0}} v(0)\right|^{2} d \mathcal{V}_{0} \\
& -\varepsilon \int_{0}^{t} \int_{\mathcal{S}} \varrho\left(\partial_{t}+v_{y} \cdot \nabla_{y}+V_{z} \partial_{z}\right)\left[\operatorname{div}^{\varphi}, \mathcal{Z}^{\alpha}\right] v \mathcal{Z}^{\alpha} \operatorname{div}^{\varphi_{\tau}} v d \mathcal{V}_{\tau} d \tau \\
\leq & -\frac{1}{2} \varepsilon \int_{\mathcal{S}} \varrho\left|\mathcal{Z}^{\alpha} \operatorname{div}^{\varphi} v(t)\right|^{2} d \mathcal{V}_{t}+\frac{1}{2} \varepsilon \int_{\mathcal{S}} \varrho_{0}\left|\mathcal{Z}^{\alpha} \operatorname{div}^{\varphi_{0}} v(0)\right|^{2} d \mathcal{V}_{0} \\
& +\Lambda_{\infty}(t) \varepsilon \int_{0}^{t}\left\|\left(\partial_{t}+v_{y} \cdot \nabla_{y}+\frac{(1-z) V_{z}}{z} Z_{3}\right)\left[\operatorname{div}^{\varphi}, \mathcal{Z}^{\alpha}\right] v\right\|\left\|\mathcal{Z}^{\alpha} \operatorname{div}^{\varphi} v\right\| d \tau \\
\leq & -\frac{1}{2} \varepsilon \int_{\mathcal{S}} \varrho\left|\mathcal{Z}^{\alpha} \operatorname{div}^{\varphi} v(t)\right|^{2} d \mathcal{V}_{t}+\frac{1}{2} \varepsilon \int_{\mathcal{S}} \varrho_{0}\left|\mathcal{Z}^{\alpha} \operatorname{div}^{\varphi_{0}} v(0)\right|^{2} d \mathcal{V}_{0} \\
& +\Lambda_{\infty}(t) \int_{0}^{t} \varepsilon\|\nabla v\|_{\mathcal{H}^{m-1}}^{2}+\varepsilon\left|\mathcal{Z}^{m} h\right|_{\frac{1}{2}}^{2} d \tau,
\end{aligned}
$$

where one has the fact $\left.V_{z}\right|_{z=0}=0$ and

$$
\left\|\frac{V_{z}}{z}\right\|_{\infty, t} \leq\left\|V_{z}\right\|_{\infty, t}+\left\|\partial_{z} V_{z}\right\|_{\infty, t} \leq \Lambda\left(\frac{1}{c_{0}},\left\|\left(v, \partial_{z} v\right)\right\|_{\infty, t}+|h|_{\mathcal{W}_{t}^{2, \infty}}\right) .
$$

Similarly, one gets that

$$
K_{2} \leq \Lambda_{\infty}(t) \int_{0}^{t}\|v\|_{\mathcal{H}^{m}}^{2}+\|\nabla v\|_{\mathcal{H}^{m-1}}^{2}+\varepsilon\left|\mathcal{Z}^{m-1} h\right|_{\frac{1}{2}}^{2} d \tau
$$


As to the boundary term $K_{3}$, one can obtain from the trace estimates (2.5) that

$$
\begin{gathered}
K_{3} \leq \Lambda_{\infty}(t) \varepsilon \int_{0}^{t}\left|\mathcal{Z} \mathcal{Z}^{\alpha} v\right|_{L^{2}\left(\mathbb{R}^{2}\right)}\left|\mathcal{Z}^{\alpha} \operatorname{div}^{\varphi} v\right|_{L^{2}\left(\mathbb{R}^{2}\right)} d \tau \\
\leq \Lambda_{\infty}(t) \varepsilon \int_{0}^{t}\left(\left\|\nabla \mathcal{Z} \mathcal{Z}^{\alpha} v\right\|^{\frac{1}{2}}\left\|\mathcal{Z} \mathcal{Z}^{\alpha} v\right\|^{\frac{1}{2}}+\left\|\mathcal{Z} \mathcal{Z}^{\alpha} v\right\|\right) \\
\cdot\left(\left\|\nabla \mathcal{Z}^{\alpha} \operatorname{div}^{\varphi} v\right\|^{\frac{1}{2}}\left\|\mathcal{Z}^{\alpha} \operatorname{div}^{\varphi} v\right\|^{\frac{1}{2}}+\left\|\mathcal{Z}^{\alpha} \operatorname{div}^{\varphi} v\right\|\right) d \tau \\
\leq \delta_{1} \varepsilon^{2} \int_{0}^{t}\left\|\nabla^{2} v\right\|_{\mathcal{H}^{m-1}}^{2} d \tau+\delta_{1} \varepsilon \int_{0}^{t}\left\|\nabla V^{m}\right\|^{2} d \tau \\
\quad+C_{\delta_{1}} \Lambda_{\infty}(t) \int_{0}^{t}\|v\|_{\mathcal{H}^{m}}^{2}+\|\nabla v\|_{\mathcal{H}^{m-1}}^{2}+\varepsilon\left|\mathcal{Z}^{m} h\right|^{2} d \tau .
\end{gathered}
$$

where (4.3.10) and Corollary 2.6 have been used.

Substituting (4.3.9), (4.3.11) and (4.3.12) into (4.3.8), one has that

$$
\begin{aligned}
& \varepsilon \int_{0}^{t} \int_{\mathcal{S}} \varrho\left(\partial_{t}^{\varphi}+v \cdot \nabla^{\varphi}\right) \mathcal{Z}^{\alpha} v \cdot \nabla^{\varphi} \mathcal{Z}^{\alpha} \operatorname{div}^{\varphi} v d \mathcal{V}_{\tau} d \tau \\
& \leq-\frac{1}{2} \varepsilon \int_{\mathcal{S}} \varrho\left|\mathcal{Z}^{\alpha} \operatorname{div}^{\varphi} v(t)\right|^{2} d \mathcal{V}_{t}+\frac{1}{2} \varepsilon \int_{\mathcal{S}} \varrho_{0}\left|\mathcal{Z}^{\alpha} \operatorname{div}^{\varphi 0} v(0)\right|^{2} d \mathcal{V}_{0}+\delta_{1} \varepsilon^{2} \int_{0}^{t}\left\|\nabla^{2} v\right\|_{\mathcal{H}^{m-1}}^{2} d \tau \\
& \quad+\delta_{1} \varepsilon \int_{0}^{t}\left\|\nabla V^{m}\right\|^{2} d \tau+C_{\delta_{1}} \Lambda_{\infty}(t) \int_{0}^{t}\|v\|_{\mathcal{H}^{m}}^{2}+\|\nabla v\|_{\mathcal{H}^{m-1}}^{2}+\left|\mathcal{Z}^{m} h\right|^{2} d \tau
\end{aligned}
$$

For the pressure term, it follows from (4.3.5) that

$$
\begin{aligned}
& \varepsilon \int_{0}^{t} \int_{\mathcal{S}} \mathcal{Z}^{\alpha} \nabla^{\varphi} p \cdot \nabla^{\varphi} \mathcal{Z}^{\alpha} \operatorname{div}^{\varphi} v d \mathcal{V}_{\tau} d \tau \\
& =\varepsilon \int_{0}^{t} \int_{\mathcal{S}} \mathcal{Z}^{\alpha} \nabla^{\varphi} p \cdot \mathcal{Z}^{\alpha} \nabla^{\varphi} \operatorname{div}^{\varphi} v d \mathcal{V}_{\tau} d \tau+\varepsilon \int_{0}^{t} \int_{\mathcal{S}} \mathcal{Z}^{\alpha} \nabla^{\varphi} p\left[\nabla^{\varphi}, \mathcal{Z}^{\alpha}\right] \operatorname{div}^{\varphi} v d \mathcal{V}_{\tau} d \tau \\
& =\varepsilon \int_{0}^{t} \int_{\mathcal{S}} \mathcal{Z}^{\alpha} \nabla^{\varphi} p \cdot\left(\left[\nabla^{\varphi}, \mathcal{Z}^{\alpha}\right] \operatorname{div}^{\varphi} v+\mathcal{Z}^{\alpha}\left(\nabla^{\varphi}\left(\frac{1}{\gamma p}\right) \partial_{t}^{\varphi} p+\nabla^{\varphi}\left(\frac{v}{\gamma p}\right) \cdot \nabla^{\varphi} p\right)\right) d \mathcal{V}_{\tau} d \tau \\
& \quad-\varepsilon \int_{0}^{t} \int_{\mathcal{S}} \mathcal{Z}^{\alpha} \nabla^{\varphi} p \cdot \mathcal{Z}^{\alpha}\left(\frac{1}{\gamma p}\left(\partial_{t}^{\varphi}+v \cdot \nabla^{\varphi}\right) \nabla^{\varphi} p\right) d \mathcal{V}_{\tau} d \tau=: L_{1}+L_{2} .
\end{aligned}
$$

Using (2.1) and (2.13), one obtains immediately that

$$
\begin{gathered}
L_{1} \leq \delta \int_{0}^{t}\|\nabla p\|_{\mathcal{H}^{m-1}}^{2} d \tau+\Lambda_{\infty}(t)\left(1+\left\|\nabla \operatorname{div}^{\varphi} v\right\|_{\infty, t}^{2}\right) \int_{0}^{t} \Lambda\left(Y_{m}\right) d \tau \\
+C_{\delta} \Lambda_{\infty}(t) \varepsilon^{2} \int_{0}^{t}\left\|\nabla^{2} v\right\|_{\mathcal{H}^{m-2}}^{2} d \tau
\end{gathered}
$$

As to $L_{2}$, note that

$$
\begin{aligned}
& \mathcal{Z}^{\alpha}\left(\frac{1}{\gamma p}\left(\partial_{t}^{\varphi}+v \cdot \nabla^{\varphi}\right) \nabla^{\varphi} p\right)=\frac{1}{\gamma p}\left(\partial_{t}^{\varphi}+v \cdot \nabla^{\varphi}\right) \mathcal{Z}^{\alpha} \nabla^{\varphi} p+\frac{V_{z}}{\gamma p}\left[\mathcal{Z}^{\alpha}, \partial_{z}\right] \nabla^{\varphi} p \\
& \quad+\sum_{\substack{\beta+\nu=\alpha \\
|\beta| \geq 1}} c_{\beta, \nu}\left(\mathcal{Z}^{\beta}\left(\frac{1}{\gamma p}\right) \mathcal{Z}^{\nu} \partial_{t} \nabla^{\varphi} p+\mathcal{Z}^{\beta}\left(\frac{v_{y}}{\gamma p}\right) \mathcal{Z}^{\nu} \nabla_{y} \nabla^{\varphi} p+\mathcal{Z}^{\beta}\left(\frac{V_{z}}{\gamma p}\right) \mathcal{Z}^{\nu} \partial_{z} \nabla^{\varphi} p\right),
\end{aligned}
$$

then, it follows from integration by parts and (2.10) that

$$
\begin{aligned}
& -\varepsilon \int_{0}^{t} \int_{\mathcal{S}} \mathcal{Z}^{\alpha} \nabla^{\varphi} p \cdot \frac{1}{\gamma p}\left(\partial_{t}^{\varphi}+v \cdot \nabla^{\varphi}\right) \mathcal{Z}^{\alpha} \nabla^{\varphi} p d \mathcal{V}_{\tau} d \tau \\
& =-\varepsilon \int_{\mathcal{S}} \frac{1}{2 \gamma p}\left|\mathcal{Z}^{\alpha} \nabla^{\varphi} p\right|^{2} d \mathcal{V}_{t}+\varepsilon \int_{\mathcal{S}} \frac{1}{2 \gamma p_{0}}\left|\mathcal{Z}^{\alpha} \nabla^{\varphi_{0}} p_{0}\right|^{2} d \mathcal{V}_{0}
\end{aligned}
$$




$$
\begin{gathered}
+\varepsilon \int_{0}^{t} \int_{\mathcal{S}}\left(\partial_{t}^{\varphi}\left(\frac{1}{2 \gamma p}\right)+\operatorname{div}^{\varphi}\left(\frac{v}{2 \gamma p}\right)\right)\left|\mathcal{Z}^{\alpha} \nabla^{\varphi} p\right|^{2} d \mathcal{V}_{t} d \tau \\
\leq-\varepsilon \int_{\mathcal{S}} \frac{1}{2 \gamma p}\left|\mathcal{Z}^{\alpha} \nabla^{\varphi} p\right|^{2} d \mathcal{V}_{t}+\varepsilon \int_{\mathcal{S}} \frac{1}{2 \gamma p_{0}}\left|\mathcal{Z}^{\alpha} \nabla^{\varphi_{0}} p_{0}\right|^{2} d \mathcal{V}_{0}+\Lambda_{\infty}(t) \int_{0}^{t} \varepsilon\|\nabla p\|_{\mathcal{H}^{m-1}}^{2}+\varepsilon\left|\mathcal{Z}^{m-1} h\right|_{\frac{1}{2}}^{2} d \tau .
\end{gathered}
$$

Since $V_{z}$ vanishes on the boundary, one has that

$$
-\varepsilon \int_{0}^{t} \int_{\mathcal{S}} \mathcal{Z}^{\alpha} \nabla^{\varphi} p \cdot \frac{V_{z}}{\gamma p}\left[\mathcal{Z}^{\alpha}, \partial_{z}\right] \nabla^{\varphi} p d \mathcal{V}_{\tau} d \tau \leq \Lambda_{\infty}(t) \int_{0}^{t} \varepsilon\|\nabla p\|_{\mathcal{H}^{m-1}}^{2}+\varepsilon\left|\mathcal{Z}^{m-1} h\right|_{\frac{1}{2}}^{2} d \tau .
$$

For the term like

$$
-\varepsilon \int_{0}^{t} \int_{\mathcal{S}} c_{\beta, \nu} \mathcal{Z}^{\alpha} \nabla^{\varphi} p \mathcal{Z}^{\beta}\left(\frac{1}{\gamma p}\right) \mathcal{Z}^{\nu} \partial_{t} \nabla^{\varphi} p
$$

where $\beta$ and $\nu$ satisfy that $\beta \neq 0$ and $\beta+\nu=\alpha$, it follows from (2.1) that

$$
\begin{gathered}
-\varepsilon \int_{0}^{t} \int_{\mathcal{S}} c_{\beta, \nu} \mathcal{Z}^{\alpha} \nabla^{\varphi} p \mathcal{Z}^{\beta}\left(\frac{1}{\gamma p}\right) \mathcal{Z}^{\nu} Z_{0} \nabla^{\varphi} p \leq \int_{0}^{t} \varepsilon\left\|\nabla^{\varphi} p\right\|_{\mathcal{H}^{m-1}}^{2} d \tau+\varepsilon \int_{0}^{t}\left\|\mathcal{Z}^{\beta-1} \mathcal{Z}\left(\frac{1}{\gamma p}\right) \mathcal{Z}^{\nu} Z_{0} \nabla^{\varphi} p\right\|^{2} d \tau \\
\leq \Lambda_{\infty}(t) \int_{0}^{t} \varepsilon\|\nabla p\|_{\mathcal{H}^{m-1}}^{2}+\|p\|_{\mathcal{H}^{m-1}}^{2}+\varepsilon\left|\mathcal{Z}^{m-1} h\right|_{\frac{1}{2}}^{2} d \tau
\end{gathered}
$$

In a similar way, one gets that

$$
-\varepsilon \int_{0}^{t} \int_{\mathcal{S}} c_{\beta, \nu} \mathcal{Z}^{\alpha} \nabla^{\varphi} p \mathcal{Z}^{\beta}\left(\frac{v_{y}}{\gamma p}\right) \mathcal{Z}^{\nu} \nabla_{y} \nabla^{\varphi} p \leq \Lambda_{\infty}(t) \int_{0}^{t} Y_{m}(\tau) d \tau .
$$

To estimate the term like

$$
-\varepsilon \int_{0}^{t} \int_{\mathcal{S}} c_{\beta, \nu} \mathcal{Z}^{\alpha} \nabla^{\varphi} p \mathcal{Z}^{\beta}\left(\frac{V_{z}}{\gamma p}\right) \mathcal{Z}^{\nu} \partial_{z} \nabla^{\varphi} p
$$

one can rewrite it as

$$
-\varepsilon \int_{0}^{t} \int_{\mathcal{S}} c_{\tilde{\beta}, \tilde{\nu}} \mathcal{Z}^{\alpha} \nabla^{\varphi} p \mathcal{Z}^{\tilde{\beta}}\left(\frac{(1-z) V_{z}}{\gamma p z}\right) Z_{3} \mathcal{Z}^{\tilde{\nu}} \nabla^{\varphi} p
$$

where $|\tilde{\beta}|+|\tilde{\nu}| \leq m-1, \tilde{\nu} \leq m-2$ and $c_{\tilde{\beta}, \tilde{\nu}}$ are smooth bounded functions depend only on $z$. Indeed, this follows from the fact that $Z_{3}((1-z) / z)=\tilde{c}(1-z) / z$ for some smooth bounded function $\tilde{c}$.

If $\tilde{\beta}=0,|\tilde{\nu}| \leq m-2$, then (4.3.10) implies that

$$
-\varepsilon \int_{0}^{t} \int_{\mathcal{S}} \mathcal{Z}^{\alpha} \nabla^{\varphi} p\left(\frac{(1-z) V_{z}}{\gamma p z}\right) Z_{3} \mathcal{Z}^{\tilde{\nu}} \nabla^{\varphi} p \leq \Lambda_{\infty}(t) \int_{0}^{t} \varepsilon\|\nabla p\|_{\mathcal{H}^{m-1}}^{2}+\varepsilon\left|\mathcal{Z}^{m-1} h\right|_{\frac{1}{2}}^{2} d \tau .
$$

When $\tilde{\beta} \neq 0$, it follows from (2.1) that

$$
\begin{aligned}
& -\varepsilon \int_{0}^{t} \int_{\mathcal{S}} \mathcal{Z}^{\alpha} \nabla^{\varphi} p \mathcal{Z}^{\tilde{\beta}}\left(\frac{(1-z) V_{z}}{\gamma p z}\right) Z_{3} \mathcal{Z}^{\tilde{\nu}} \nabla^{\varphi} p \\
\leq & \varepsilon\left\{\int_{0}^{t}\left\|\mathcal{Z}^{\alpha} \nabla^{\varphi} p\right\|^{2} d \tau\right\}^{\frac{1}{2}}\left\{\int_{0}^{t}\left\|\mathcal{Z}^{\tilde{\beta}}\left(\frac{(1-z) V_{z}}{\gamma p z}\right) Z_{3} \mathcal{Z}^{\tilde{\nu}} \nabla^{\varphi} p\right\|^{2} d \tau\right\}^{\frac{1}{2}} \\
\leq & \Lambda_{\infty}(t) \varepsilon\left\{\int_{0}^{t}\left\|\nabla^{\varphi} p\right\|_{\mathcal{H}^{m-1}}^{2} d \tau\right\}^{\frac{1}{2}}\left\{\int_{0}^{t}\left\|\nabla^{\varphi} p\right\|_{\mathcal{H}^{m-1}}^{2}+\left\|\frac{(1-z) V_{z}}{\gamma p z}\right\|_{\mathcal{H}^{m-1}}^{2} d \tau\right\}^{\frac{1}{2}},
\end{aligned}
$$

where one has used (3.22), (3.23) and (4.3.10). Using (2.1) and the Hardy inequality (Lemma 8.4 in [32), one gets that

$$
\begin{aligned}
\int_{0}^{t}\left\|\frac{(1-z) V_{z}}{\gamma p z}\right\|_{\mathcal{H}^{m-1}}^{2} d \tau & \leq \Lambda_{\infty}(t) \int_{0}^{t}\|p\|_{\mathcal{H}^{m-1}}^{2}+\left\|\frac{1-z}{z} V_{z}\right\|_{\mathcal{H}^{m-1}}^{2} d \tau \\
& \leq \Lambda_{\infty}(t) \int_{0}^{t}\|p\|_{\mathcal{H}^{m-1}}^{2}+\left\|V_{z}\right\|_{\mathcal{H}^{m-1}}^{2}+\left\|\nabla V_{z}\right\|_{\mathcal{H}^{m-1}}^{2} d \tau
\end{aligned}
$$


It follows from (3.16) that

$$
\int_{0}^{t}\left\|\nabla V_{z}\right\|_{\mathcal{H}^{m-1}}^{2} d \tau \leq \Lambda_{\infty}(t) \int_{0}^{t}\|v\|_{\mathcal{H}^{m-1}}^{2}+\|\nabla v\|_{\mathcal{H}^{m-1}}^{2}+\left|\mathcal{Z}^{m} h\right|_{\frac{1}{2}}^{2} d \tau
$$

Combining (4.3.21)-(4.3.24) leads to that

$$
-\varepsilon \int_{0}^{t} \int_{\mathcal{S}} \mathcal{Z}^{\alpha} \nabla^{\varphi} p \mathcal{Z}^{\tilde{\beta}}\left(\frac{(1-z) V_{z}}{\gamma p z}\right) Z_{3} \mathcal{Z}^{\tilde{\nu}} \nabla^{\varphi} p \leq \Lambda_{\infty}(t) \int_{0}^{t} Y_{m}(\tau)+\varepsilon\left|\mathcal{Z}^{m} h\right|_{\frac{1}{2}}^{2} d \tau .
$$

Substituting (4.3.15), (4.3.17)-(4.3.20) and (4.3.25) into (4.3.14) yields that

$$
\begin{aligned}
& \varepsilon \int_{0}^{t} \int_{\mathcal{S}} \mathcal{Z}^{\alpha} \nabla^{\varphi} p \nabla^{\varphi} \mathcal{Z}^{\alpha} \operatorname{div}^{\varphi} v d \mathcal{V}_{\tau} d \tau \\
& \leq-\varepsilon \int_{\mathcal{S}} \frac{1}{2 \gamma p}\left|\mathcal{Z}^{\alpha} \nabla^{\varphi} p\right|^{2} d \mathcal{V}_{t}+\varepsilon \int_{\mathcal{S}} \frac{1}{2 \gamma p_{0}}\left|\mathcal{Z}^{\alpha} \nabla^{\varphi_{0}} p_{0}\right|^{2} d \mathcal{V}_{0}+C_{\delta} \Lambda_{\infty}(t) \varepsilon^{2} \int_{0}^{t}\left\|\nabla^{2} v\right\|_{\mathcal{H}^{m-2}}^{2} d \tau \\
& \quad+\delta \int_{0}^{t}\left\|\nabla^{\varphi} p\right\|_{\mathcal{H}^{m-1}}^{2} d \tau+\Lambda_{\infty}(t)\left(1+\left\|\nabla \operatorname{div}^{\varphi} v\right\|_{\infty, t}^{2}\right) \int_{0}^{t} Y_{m}(\tau) d \tau
\end{aligned}
$$

Now, we estimate the RHS of (4.3.7). It follows from (2.2) (3.22) and (3.25) that

$$
\begin{gathered}
J_{1}=(2 \mu+\lambda) \varepsilon^{2} \int_{0}^{t} \int_{\mathcal{S}}\left|\nabla^{\varphi} \mathcal{Z}^{\alpha} \operatorname{div}^{\varphi} v\right|^{2} d \mathcal{V}_{\tau} d \tau+(2 \mu+\lambda) \varepsilon^{2} \int_{0}^{t} \int_{\mathcal{S}}\left[\mathcal{Z}^{\alpha}, \nabla^{\varphi}\right] \operatorname{div}^{\varphi} v \cdot \nabla^{\varphi} \mathcal{Z}^{\alpha} \operatorname{div}^{\varphi} v d \mathcal{V}_{\tau} d \tau \\
\geq \frac{2 \mu+\lambda}{2} \varepsilon^{2} \int_{0}^{t} \int_{\mathcal{S}}\left|\nabla^{\varphi} \mathcal{Z}^{\alpha} \operatorname{div}^{\varphi} v\right|^{2} d \mathcal{V}_{\tau} d \tau-\Lambda_{\infty}(t)\left(1+\left\|\nabla \operatorname{div}^{\varphi} v\right\|_{\infty, t}^{2}\right) \int_{0}^{t}\left|\mathcal{Z}^{m-1} h\right|_{\frac{1}{2}}^{2} d \tau \\
-\Lambda_{\infty}(t) \varepsilon^{2} \int_{0}^{t}\left\|\nabla^{2} v\right\|_{\mathcal{H}^{m-2}}^{2} d \tau
\end{gathered}
$$

and

$$
\begin{aligned}
\left|J_{3}\right| & \leq \frac{1}{8}(2 \mu+\lambda) \varepsilon^{2} \int_{0}^{t}\left\|\nabla^{\varphi} \mathcal{Z}^{m-1} \operatorname{div}^{\varphi} v\right\|^{2} d \tau+C \int_{0}^{t}\left\|\mathcal{C}_{M}^{\alpha}(p)\right\|^{2} d \tau \\
& \leq \frac{1}{8}(2 \mu+\lambda) \varepsilon^{2} \int_{0}^{t}\left\|\nabla \mathcal{Z}^{m-1} \operatorname{div}^{\varphi} v\right\|^{2} d \tau+\Lambda_{\infty}(t) \int_{0}^{t} Y_{m}(\tau) d \tau .
\end{aligned}
$$

Using integration by parts, one can obtain from (2.12) and the trace estimates (2.5) that

$$
\begin{aligned}
& \left|J_{2}\right|=\left|\mu \varepsilon^{2} \int_{0}^{t} \int_{\mathcal{S}} \mathcal{Z}^{\alpha}\left(\nabla^{\varphi} \times\left(\nabla^{\varphi} \times v\right)\right) \cdot \nabla^{\varphi} \mathcal{Z}^{\alpha} \operatorname{div}^{\varphi} v d \mathcal{V}_{\tau} d \tau\right| \\
& \leq \frac{1}{8}(2 \mu+\lambda) \varepsilon^{2} \int_{0}^{t}\left\|\nabla^{\varphi} \mathcal{Z}^{\alpha} \operatorname{div}^{\varphi} v\right\|^{2} d \tau+\Lambda_{0} \int_{0}^{t} \varepsilon^{2}\left\|\nabla^{2} v\right\|_{\mathcal{H}^{m-2}}^{2}+\Lambda_{\infty}(t) \int_{0}^{t} Y_{m}(\tau) d \tau \\
& \quad+\left|\mu \varepsilon^{2} \int_{0}^{t} \int_{\mathcal{S}}\left(\nabla^{\varphi} \times \mathcal{Z}^{\alpha}\left(\nabla^{\varphi} \times v\right)\right) \cdot \nabla^{\varphi} \mathcal{Z}^{\alpha} \operatorname{div}^{\varphi} v d \mathcal{V}_{\tau} d \tau\right| \\
& \leq \frac{1}{8}(2 \mu+\lambda) \varepsilon^{2} \int_{0}^{t}\left\|\nabla^{\varphi} \mathcal{Z}^{\alpha} \operatorname{div}^{\varphi} v\right\|^{2} d \tau+\Lambda_{0} \varepsilon^{2} \int_{0}^{t}\left\|\nabla^{2} v\right\|_{\mathcal{H}^{m-2}}^{2}+\Lambda_{\infty}(t) \int_{0}^{t} Y_{m}(\tau) d \tau \\
& \quad+\mu \varepsilon^{2}\left|\int_{0}^{t} \int_{z=0}\left(\mathbf{N} \times \mathcal{Z}^{\alpha}\left(\nabla^{\varphi} \times v\right)\right) \cdot\left(\nabla_{y}, 0\right) \mathcal{Z}^{\alpha} \operatorname{div}^{\varphi} v d y d \tau\right| \\
& \leq \frac{1}{8}(2 \mu+\lambda) \varepsilon^{2} \int_{0}^{t}\left\|\nabla^{\varphi} \mathcal{Z}^{\alpha} \operatorname{div}^{\varphi} v\right\|^{2} d \tau+\Lambda_{0} \int_{0}^{t} \varepsilon^{2}\left\|\nabla^{2} v\right\|_{\mathcal{H}^{m-2}}^{2}+\Lambda_{\infty}(t) \int_{0}^{t} Y_{m}(\tau) d \tau \\
& \quad+\mu \varepsilon^{2} \int_{0}^{t}\left|\left(\mathbf{N} \times \mathcal{Z}^{\alpha}\left(\nabla^{\varphi} \times v\right)\right)\right|_{\frac{1}{2}} \cdot\left|\mathcal{Z}^{\alpha} \operatorname{div}^{\varphi} v\right|_{\frac{1}{2}} d \tau \\
& \leq \frac{1}{4}(2 \mu+\lambda) \varepsilon^{2} \int_{0}^{t}\left\|\nabla^{\varphi} \mathcal{Z}^{m-1} \operatorname{div}^{\varphi} v\right\|^{2} d \tau+\delta_{1} \varepsilon^{2} \int_{0}^{t}\left\|\nabla^{2} v\right\|_{\mathcal{H}^{m-1}}^{2} d \tau+\frac{\Lambda_{0}}{\delta_{1}} \varepsilon^{2} \int_{0}^{t}\left\|\nabla V^{m}\right\|^{2} d \tau \\
& \quad+C_{\delta_{1}} \Lambda_{\infty}(t) \int_{0}^{t} Y_{m}(\tau)+\varepsilon^{2}\left\|\nabla^{2} v\right\|_{\mathcal{H}^{m-2}}^{2}+\varepsilon\left|\mathcal{Z}^{m} h\right|_{\frac{1}{2}}^{2} d \tau .
\end{aligned}
$$


Combining (4.3.7), (4.3.13), (4.3.26)-(4.3.29) and summing over $\alpha(|\alpha| \leq m-1)$ complete the proof of this lemma.

In order to close the estimate in Lemma 4.7, it remains to bound $\varepsilon^{2} \int_{0}^{t}\left\|\nabla^{2} v\right\|_{\mathcal{H}^{m-2}}^{2} d \tau$. We first estimate $\int_{0}^{t}\left\|\nabla \mathcal{Z}^{m-2} \operatorname{div}^{\varphi} u\right\|^{2} d \tau$ by using of the mass equation (4.3.4). It should be mentioned that, in general, it is difficult to derive an uniform estimate for $\int_{0}^{t}\left\|\mathcal{Z}^{m-2} \partial_{z z} u\right\|^{2} d \tau$ due to the possible appearance of boundary layers. However, one can expect the estimate of $\int_{0}^{t}\left\|\nabla \mathcal{Z}^{m-2} \operatorname{div}^{\varphi} u\right\|^{2} d \tau$ due to weaker boundary layer for $\operatorname{div} u$. Moreover, this estimate is very helpful to bound $\varepsilon \int_{0}^{t}\left\|\nabla^{2} v\right\|_{\mathcal{H}^{m-2}}^{2} d \tau$, which will be used later. Precisely, we can prove the following lemma.

Lemma 4.8 For $m \geq 2$, it holds that

$$
\int_{0}^{t}\left\|\nabla \mathcal{Z}^{m-2} d i v^{\varphi} v\right\|^{2} d \tau \leq \Lambda_{\infty}(t) \int_{0}^{t}\|\nabla p(\tau)\|_{\mathcal{H}^{m-1}}^{2}+Y_{m}(\tau) d \tau,
$$

and

$$
\varepsilon^{2} \int_{0}^{t}\left\|\nabla^{2} v\right\|_{\mathcal{H}^{m-2}}^{2} d \tau+\varepsilon^{2} \int_{0}^{t}\left\|\nabla^{\varphi} \nabla^{\varphi} v\right\|_{\mathcal{H}^{m-2}}^{2} d \tau \leq \Lambda_{\infty}(t) \int_{0}^{t} Y_{m}(\tau) d \tau .
$$

Proof. For $|\alpha| \leq m-2, m \geq 2$, it follows from (4.3.4) that

$$
\begin{aligned}
\nabla \mathcal{Z}^{\alpha} \operatorname{div}^{\varphi} v= & -\nabla\left(\frac{1}{\gamma p}\left(\partial_{t}+v_{y} \cdot \nabla_{y}+V_{z} \partial_{z}\right) \mathcal{Z}^{\alpha} p\right) \\
& -\nabla\left(\frac{1}{\gamma p}\left(\left[\mathcal{Z}^{\alpha}, v_{y}\right] \nabla_{y} p\right)-\nabla\left(\left[\mathcal{Z}^{\alpha}, \frac{1}{\gamma p}\right]\left(\partial_{t} p+v_{y} \cdot \nabla_{y} p\right)\right)\right. \\
& -\nabla\left(\frac{1}{\gamma p}\left(\left[\mathcal{Z}^{\alpha}, V_{z}\right] \partial_{z} p+V_{z}\left[\mathcal{Z}^{\alpha}, \partial_{z}\right] p\right)+\left[\mathcal{Z}^{\alpha}, \frac{1}{\gamma p}\right]\left(V_{z} \partial_{z} p\right)\right)=: \sum_{i=1}^{3} J_{i}
\end{aligned}
$$

It follows immediately from (2.1) $-(2.2)$ and the fact $\left.V_{z}\right|_{z=0}=0$ that

$$
\int_{0}^{t} \int_{\mathcal{S}}\left|J_{1}\right|^{2} d \mathcal{V}_{\tau} d \tau \leq \Lambda_{\infty}(t) \int_{0}^{t}\|(p, \nabla p)\|_{\mathcal{H}^{m-1}}^{2}
$$

and

$$
\int_{0}^{t} \int_{\mathcal{S}}\left|J_{2}\right|^{2} d \mathcal{V}_{\tau} d \tau \leq \Lambda_{\infty}(t) \int_{0}^{t}\|(p, v)\|_{\mathcal{H}^{m-2}}^{2}+\|(\nabla p, \nabla v)\|_{\mathcal{H}^{m-2}}^{2} d \tau
$$

To estimate $J_{3}$, one first notes that

$$
\nabla\left(\left[\mathcal{Z}^{\alpha}, V_{z}\right] \partial_{z} p\right)=\sum_{\substack{\beta+\nu=\alpha \\|\beta| \geq 1}} c_{\beta, \nu}\left(\nabla \mathcal{Z}^{\beta} V_{z} \mathcal{Z}^{\nu} \partial_{z} p+\mathcal{Z}^{\beta} V_{z} \nabla \mathcal{Z}^{\nu} \partial_{z} p\right)
$$

Due to (2.1) and (4.3.24), one can obtain that

$$
\begin{aligned}
\int_{0}^{t}\left\|\nabla \mathcal{Z}^{\beta} V_{z} \mathcal{Z}^{\nu} \partial_{z} p\right\|^{2} d \tau & \leq \sum_{|\tilde{\beta}| \leq m-2} \int_{0}^{t}\left\|\mathcal{Z}^{\tilde{\beta}} \nabla V_{z} \mathcal{Z}^{\nu} \partial_{z} p\right\|^{2} d \tau \\
& \leq \Lambda_{\infty}(t) \int_{0}^{t}\left\|\nabla V_{z}\right\|_{\mathcal{H}^{m-2}}^{2}+\left\|\partial_{z} p\right\|_{\mathcal{H}^{m-2}}^{2} d \tau \\
& \leq \Lambda_{\infty}(t) \int_{0}^{t}\|v\|_{\mathcal{H}^{m-2}}^{2}+\|\nabla(v, p)\|_{\mathcal{H}^{m-2}}^{2}+|h|_{\mathcal{H}^{m}}^{2} d \tau
\end{aligned}
$$

On the other hand, it follows from (2.1), Hardy inequality, 3.25) and (4.3.24) that

$$
\begin{aligned}
& \int_{0}^{t}\left\|\mathcal{Z}^{\beta} V_{z} \nabla \mathcal{Z}^{\nu} \partial_{z} p\right\|^{2} d \tau \leq \int_{0}^{t}\left\|\mathcal{Z}^{\beta}\left(\frac{1-z}{z} V_{z}\right) \mathcal{Z Z}^{\nu} \partial_{z} p\right\|^{2} d \tau \\
& \leq \Lambda_{\infty}(t) \int_{0}^{t}\left\|\frac{1-z}{z} V_{z}\right\|_{\mathcal{H}^{m-2}}^{2}+\left\|\partial_{z} p\right\|_{\mathcal{H}^{m-1}}^{2} d \tau \leq \Lambda_{\infty}(t) \int_{0}^{t}\left\|\left(V_{z}, \nabla V_{z}\right)\right\|_{\mathcal{H}^{m-2}}^{2}+\left\|\partial_{z} p\right\|_{\mathcal{H}^{m-1}}^{2} d \tau \\
& \leq \Lambda_{\infty}(t) \int_{0}^{t}\|(v, \nabla v)\|_{\mathcal{H}^{m-2}}^{2}+\|\nabla p\|_{\mathcal{H}^{m-1}}^{2}+|h|_{\mathcal{H}^{m}}^{2} d \tau
\end{aligned}
$$


Thus,

$$
\begin{aligned}
& \int_{0}^{t} \int_{\mathcal{S}}\left|\nabla\left(\frac{1}{\gamma p}\left[\mathcal{Z}^{\alpha}, V_{z}\right] \partial_{z} p\right)\right|^{2} d \mathcal{V}_{\tau} d \tau \leq \Lambda_{\infty}(t) \int_{0}^{t} \int_{\mathcal{S}}\left|\left[\mathcal{Z}^{\alpha}, V_{z}\right] \partial_{z} p\right|^{2}+\left|\nabla\left(\left[\mathcal{Z}^{\alpha}, V_{z}\right] \partial_{z} p\right)\right|^{2} d \mathcal{V}_{\tau} d \tau \\
& \leq \Lambda_{\infty}(t) \int_{0}^{t}\left\|V_{z}\right\|_{\mathcal{H}^{m-2}}^{2}+\left\|\partial_{z} p\right\|_{\mathcal{H}^{m-3}}^{2}+\left\|\nabla \mathcal{Z}^{\beta} V_{z} \mathcal{Z}^{\nu} \partial_{z} p\right\|^{2}+\left\|\mathcal{Z}^{\beta} V_{z} \nabla \mathcal{Z}^{\nu} \partial_{z} p\right\|^{2} d \tau \\
& \leq \Lambda_{\infty}(t) \int_{0}^{t}\|(v, \nabla v)\|_{\mathcal{H}^{m-2}}^{2}+\|\nabla p\|_{\mathcal{H}^{m-1}}^{2}+|h|_{\mathcal{H}^{m}}^{2} d \tau
\end{aligned}
$$

Similarly,

$$
\int_{0}^{t} \int_{\mathcal{S}}\left|\nabla\left(\left[\mathcal{Z}^{\alpha}, \frac{1}{\gamma p}\right]\right)\left(V_{z} \partial_{z} p\right)\right|^{2} d \mathcal{V}_{\tau} d \tau \leq \Lambda_{\infty}(t) \int_{0}^{t}\|(v, p, \nabla v, \nabla p)\|_{\mathcal{H}^{m-2}}^{2}+|h|_{\mathcal{H}^{m}}^{2} d \tau
$$

Finally, it follows from (2.2), (3.23) and (4.3.10) that

$$
\int_{0}^{t} \int_{\mathcal{S}}\left|\nabla\left(\frac{1}{\gamma p} V_{z}\left[\mathcal{Z}^{\alpha}, \partial_{z}\right] p\right)\right|^{2} d \mathcal{V}_{\tau} d \tau \leq \Lambda_{\infty}(t) \int_{0}^{t}\|\nabla p\|_{\mathcal{H}^{m-2}}^{2} d \tau .
$$

Combining (4.3.37), (4.3.38) and (4.3.39), one gets that

$$
\int_{0}^{t} \int_{\mathcal{S}}\left|J_{3}\right|^{2} d \mathcal{V}_{\tau} d \tau \leq \Lambda_{\infty}(t) \int_{0}^{t}\|(v, \nabla v)\|_{\mathcal{H}^{m-2}}^{2}+\|(p, \nabla p)\|_{\mathcal{H}^{m-1}}^{2}+|h|_{\mathcal{H}^{m}}^{2} d \tau
$$

Then, (4.3.30) follows from (4.3.32)-(4.3.34) and 4.3.40).

By (1.31) 2 , 4.3.30) and Corollary 2.6] one obtains that

$$
\begin{aligned}
\varepsilon^{2} \int_{0}^{t}\left\|\partial_{z}^{2} v\right\|_{\mathcal{H}^{m-2}}^{2} d \tau & \leq \varepsilon^{2} \int_{0}^{t}\left\|\nabla \mathcal{Z}^{m-2} \operatorname{div}^{\varphi} v\right\| d \tau+\Lambda_{\infty}(t) \int_{0}^{t} Y_{m}(\tau) d \tau \\
& \leq \Lambda_{\infty}(t) \int_{0}^{t} Y_{m}(\tau) d \tau
\end{aligned}
$$

Then, 4.3.31) follows immediately. Therefore, the lemma is proved.

Now we can estimate $\int_{0}^{t}\|\nabla p\|_{\mathcal{H}^{m-1}}^{2} d \tau$ in the following lemma.

Lemma 4.9 For every $m \geq 1$, it holds that

$$
\int_{0}^{t}\|\nabla p\|_{\mathcal{H}^{m-1}}^{2} d \tau \leq \Lambda_{0} \varepsilon^{2} \int_{0}^{t}\left\|\nabla^{2} v\right\|_{\mathcal{H}^{m-1}}^{2} d \tau+\Lambda_{\infty}(t) \int_{0}^{t} \Lambda\left(Y_{m}(\tau)\right)+\varepsilon\left|\mathcal{Z}^{m-1} \nabla_{y} h\right|_{\frac{1}{2}}^{2} d \tau .
$$

Proof. For any $|\alpha| \leq m-1$, it follows from $(4.3 .4)_{2},(2.1),(2.2)$, and (2.12) that

$$
\begin{aligned}
& \int_{0}^{t}\left\|\mathcal{Z}^{\alpha} \nabla^{\varphi} p\right\|^{2} d \tau \lesssim \int_{0}^{t}\left\|\varrho\left(\partial_{t}+v_{y} \cdot \nabla_{y}+V_{z} \partial_{z}\right) \mathcal{Z}^{\alpha} v\right\|^{2} d \tau \\
& \quad+\varepsilon^{2} \int_{0}^{t}\left\|\operatorname{div}^{\varphi} S^{\varphi} v\right\|_{\mathcal{H}^{m-1}}^{2}+\left\|\nabla^{\varphi} \operatorname{div}^{\varphi} v\right\|_{\mathcal{H}^{m-1}}^{2} d \tau+\int_{0}^{t}\left\|\left[\mathcal{Z}^{\alpha}, \varrho\right]\left(\partial_{t} v+v_{y} \cdot \nabla_{y} v+V_{z} \partial_{z} v\right)\right\|^{2} d \tau \\
& \quad+\int_{0}^{t}\left\|\varrho\left(\left[\mathcal{Z}^{\alpha}, v_{y}\right] \nabla_{y} v+\left[\mathcal{Z}^{\alpha}, V_{z}\right] \partial_{z} v+V_{z}\left[\mathcal{Z}^{\alpha}, \partial_{z}\right] v\right)\right\|^{2} d \tau \\
& \leq \Lambda_{\infty}(t) \int_{0}^{t}\|v\|_{\mathcal{H}^{m}}^{2}+\|\nabla v\|_{\mathcal{H}^{m-2}}^{2}+|h|_{\mathcal{H}^{m}}^{2}+\|p\|_{\mathcal{H}^{m}}^{2} d \tau+C \varepsilon^{2} \int_{0}^{t}\left\|\nabla^{\varphi} \nabla^{\varphi} v\right\|_{\mathcal{H}^{m-1}}^{2} d \tau \\
& \leq \Lambda_{0} \varepsilon^{2} \int_{0}^{t}\left\|\nabla^{2} v\right\|_{\mathcal{H}^{m-1}}^{2} d \tau+\Lambda_{\infty}(t) \int_{0}^{t} Y_{m}(\tau)+\varepsilon\left|\mathcal{Z}^{m-1} \nabla_{y} h\right|_{\frac{1}{2}}^{2} d \tau
\end{aligned}
$$

which combining (2.11) yields the proof of this lemma.

Since $\operatorname{div}^{\varphi} v$ is not free, the following estimate is the key to estimate $\int_{0}^{t}\left\|\partial_{z} v\right\|_{\mathcal{H}^{m-1}}^{2} d \tau$ : 
Lemma 4.10 For $m \geq 6$, it holds that

$$
\left\|d i v^{\varphi} v\right\|_{\mathcal{H}^{m-1}}^{2} \leq \Lambda\left(\frac{1}{c_{0}}, \mathcal{Q}_{m}(t)+\mathcal{Q}(t)\right)
$$

where $\mathcal{Q}_{m}(t) \doteq\|(v, p)(t)\|_{\mathcal{H}^{m}}^{2}+\|(\nabla v, \nabla p)(t)\|_{\mathcal{H}^{m-2}}^{2}+|h(t)|_{\mathcal{H}^{m}}^{2}$.

Proof. Applying $\mathcal{Z}^{\alpha}$ with $|\alpha| \leq m-1$ to (4.3.5) gives that

$$
\mathcal{Z}^{\alpha} \operatorname{div}^{\varphi} v=-\mathcal{Z}^{\alpha}\left(\frac{\partial_{t} p}{\gamma p}+\frac{v_{y} \cdot \nabla_{y} p}{\gamma p}\right)-\mathcal{Z}^{\alpha}\left(\frac{V_{z} \partial_{z} p}{\gamma p}\right) .
$$

Note that

$$
\mathcal{Z}^{\alpha}\left(\frac{\partial_{t} p}{p}\right)=\sum_{|\beta|=0}^{|\alpha|} \mathcal{Z}^{\beta}\left(\frac{1}{p}\right) \mathcal{Z}^{\nu} \partial_{t} p
$$

then it follows that

$$
\left\|\frac{\partial_{t} p}{p}\right\|_{\mathcal{H}^{m-1}}^{2} \leq \sum_{\substack{|\beta|=0 \\|\beta|+|\nu| \leq m-1}}^{|\beta|=\left[\frac{m}{2}\right]}\left\|\mathcal{Z}^{\beta}\left(\frac{1}{p}\right)\right\|_{\infty}^{2}\left\|\mathcal{Z}^{\nu} \partial_{t} p\right\|^{2}+\sum_{\substack{|\beta|=1+\left[\frac{m}{2}\right] \\|\beta|+|\nu| \leq m-1}}^{|\beta|=m-1}\left\|\mathcal{Z}^{\nu} \partial_{t} p\right\|_{\infty}^{2}\left\|\mathcal{Z}^{\beta}\left(\frac{1}{p}\right)\right\|^{2} .
$$

For the $L^{\infty}$ norm, it holds, for $|\beta| \leq\left[\frac{m}{2}\right]$, that

$$
\sum_{|\beta| \leq\left[\frac{m}{2}\right]}\left\|\mathcal{Z}^{\beta} p\right\|_{L^{\infty}}^{2} \lesssim \sum_{|\beta| \leq\left[\frac{m}{2}\right]}\left\|\nabla \mathcal{Z}^{\beta} p\right\|_{1}\left\|\mathcal{Z}^{\beta} p\right\|_{2} \lesssim\|p\|_{\mathcal{H}^{\left[\frac{m}{2}\right]+2}}^{2}+\|\nabla p\|_{\mathcal{H}^{\left[\frac{m}{2}\right]+1}}^{2}
$$

Thus, for $m \geq 6$, that is $\left[\frac{m}{2}\right]+2 \leq m$ and $\left[\frac{m}{2}\right]+1 \leq m-2$, one gets that

$$
\sum_{|\beta| \leq\left[\frac{m}{2}\right]}\left\|\mathcal{Z}^{\beta} p\right\|_{\infty}^{2} \leq \mathcal{Q}_{m}(t)
$$

which implies that

$$
\left\|\frac{\partial_{t} p}{p}\right\|_{\mathcal{H}^{m-1}}^{2} \leq \Lambda\left(\frac{1}{c_{0}}, \mathcal{Q}_{m}(t)+\mathcal{Q}(t)\right), \quad m \geq 6
$$

Similarly, one has that

$$
\sum_{|\beta| \leq\left[\frac{m}{2}\right]}\left\|\mathcal{Z}^{\beta} v\right\|_{\infty}^{2} \leq \Lambda\left(\frac{1}{c_{0}}, \mathcal{Q}_{m}(t)+\mathcal{Q}(t)\right), \quad m \geq 6,
$$

which yields that, for $m \geq 6$,

$$
\begin{aligned}
\left\|\frac{v_{y} \cdot \nabla_{y} p}{p}(t)\right\|_{\mathcal{H}^{m-1}}^{2} & \leq \sum_{\substack{|\beta|=0 \\
|\beta|+|\nu| \leq m-1}}^{|\beta|=\left[\frac{m}{2}\right]}\left\|\mathcal{Z}^{\beta}\left(\frac{v}{p}\right)\right\|_{\infty}^{2}\left\|\mathcal{Z}^{\alpha} \nabla_{y} p\right\|^{2}+\sum_{\substack{|\beta|=1+\left[\frac{m}{2}\right] \\
|\beta|+|\nu| \leq m-1}}^{|\beta|=m-1}\left\|\mathcal{Z}^{\nu} \nabla_{y} p\right\|_{\infty}^{2}\left\|\mathcal{Z}^{\beta}\left(\frac{v}{p}\right)\right\|^{2} \\
& \leq \Lambda\left(\frac{1}{c_{0}}, \mathcal{Q}_{m}(t)+\mathcal{Q}(t)\right)
\end{aligned}
$$

Additional care is needed to estimate $\left\|\frac{V_{z} \partial_{z} p}{p}(t)\right\|_{\mathcal{H}^{m-1}}$ since it involves $\partial_{z} p$. Rewrite this term as

$$
\mathcal{Z}^{\alpha}\left(\frac{V_{z} \partial_{z} p}{p}\right)=\frac{V_{z}}{p} \mathcal{Z}^{\alpha} \partial_{z} p+\partial_{z} p \mathcal{Z}^{\alpha}\left(\frac{V_{z}}{p}\right)+\sum_{\substack{|\beta|=1 \\ \beta+\nu=\alpha}}^{m-2} c_{k} \mathcal{Z}^{\beta}\left(\frac{V_{z}}{p}\right) \mathcal{Z}^{\nu} \partial_{z} p
$$

Then, it follows from (3.25) that

$$
\left\|\frac{V_{z}}{p} \mathcal{Z}^{\alpha} \partial_{z} p\right\|^{2} \leq\left\|\frac{(1-z) V_{z}}{z p}\right\|_{\infty}^{2}\|p\|_{\mathcal{H}^{m}}^{2} \leq \Lambda\left(\frac{1}{c_{0}}, \mathcal{Q}(t)+\mathcal{Q}_{m}(t)\right)
$$




$$
\left\|\partial_{z} p \mathcal{Z}^{\alpha}\left(\frac{V_{z}}{p}\right)(t)\right\|^{2} \leq \Lambda\left(\frac{1}{c_{0}}, \mathcal{Q}(t)+\mathcal{Q}_{m}(t)\right)
$$

and

$$
\begin{aligned}
& \sum_{\substack{|\beta|=1 \\
\beta+\nu=\alpha}}^{|\beta|=m-2}\left\|\mathcal{Z}^{\beta}\left(\frac{V_{z}}{p}\right) \mathcal{Z}^{\nu} \partial_{z} p\right\|^{2} \\
& \leq\left\|\mathcal{Z}\left(\frac{V_{z}}{p}\right)\right\|_{\infty}^{2}\left\|\partial_{z} p\right\|_{\mathcal{H}^{m-2}}^{2}+\left\|\mathcal{Z} \partial_{z} p\right\|_{L^{\infty}}^{2}\left\|\frac{V_{z}}{p}\right\|_{\mathcal{H}^{m-2}}^{2}+\sum_{\substack{|\beta|=2 \\
\beta+\nu=\alpha}}^{|\beta|=m-3}\left\|\mathcal{Z}^{\beta}\left(\frac{V_{z}}{p}\right)\right\|_{\infty}^{2}\left\|\mathcal{Z}^{\nu} \partial_{z} p\right\|^{2} \\
& \leq \Lambda\left(\frac{1}{c_{0}}, \mathcal{Q}_{m}(t)+\mathcal{Q}(t)\right)+\left\|\frac{V_{z}}{p}\right\|_{\mathcal{H}^{m-3, \infty}}^{2}\left\|\partial_{z} p\right\|_{\mathcal{H}^{m-3}}^{2} \\
& \leq \Lambda\left(\frac{1}{c_{0}}, \mathcal{Q}_{m}(t)+\mathcal{Q}(t)\right) .
\end{aligned}
$$

Collecting the inequalities (4.3.45), (4.3.50), (4.3.52)-(4.3.56) shows (4.3.44). Therefore, the proof of this lemma is completed.

\subsection{Normal derivative estimates: Part I}

In this subsection, we will focus on the estimates of normal derivative of $v$. To close the a priori estimates, one needs to bound $\int_{0}^{t}\left\|\partial_{z} v\right\|_{\mathcal{H}^{m-1}}^{2} d \tau, \varepsilon \int_{0}^{t}\left\|\partial_{z}^{2} v\right\|_{\mathcal{H}^{m-2}}^{2} d \tau$ and $\varepsilon^{2} \int_{0}^{t}\left\|\partial_{z}^{2} v\right\|_{\mathcal{H}^{m-1}}^{2} d \tau$ in this and next subsection. Since

$$
\partial_{z} v=\left(\partial_{z} v \cdot \mathbf{n}\right) \mathbf{n}+\Pi\left(\partial_{z} v\right)
$$

it suffices to estimate $\partial_{z} v \cdot \mathbf{n}$ and $\Pi\left(\partial_{z} v\right)$. First, one can bound the normal component of $\partial_{z} v$ in terms of $\operatorname{div}^{\varphi} v$ as follows:

Lemma 4.11 For any $k \in \mathbb{N}$, it holds that

$$
\int_{0}^{t}\left\|\partial_{z} v \cdot \mathbf{n}\right\|_{\mathcal{H}^{k}}^{2} \leq \Lambda_{0} \int_{0}^{t}\left\|d i v^{\varphi} v\right\|_{\mathcal{H}^{k}}^{2}+\left\|V^{k+1}\right\|^{2} d \tau+\Lambda_{\infty}(t) \int_{0}^{t}\left|\mathcal{Z}^{k} h\right|_{\frac{1}{2}}^{2} d \tau
$$

Proof. It follows from the definition of $\partial_{i}^{\varphi}$ that

$$
\operatorname{div}^{\varphi} v=\partial_{1}^{\varphi} v_{1}+\partial_{2}^{\varphi} v_{2}+\partial_{3}^{\varphi} v_{3}=\frac{1}{\partial_{z} \varphi} \partial_{z} v \cdot \mathbf{N}+\partial_{1} v_{1}+\partial_{2} v_{2} .
$$

which yields that

$$
\partial_{z} v \cdot \mathbf{n}=\frac{\partial_{z} \varphi}{\sqrt{1+\left|\nabla_{y} \varphi\right|^{2}}}\left(\operatorname{div}^{\varphi} v-\partial_{1} v_{1}-\partial_{2} v_{2}\right)
$$

Hence, (4.4.2) follows from (2.1), Lemma 2.4 and (4.0.5).

Next, we will estimate the tangential components of $\partial_{z} v$. Note that

$$
\nabla^{\varphi} v \mathbf{N}=-\partial_{1} \varphi \partial_{1}^{\varphi} v-\partial_{2} \varphi \partial_{2}^{\varphi} v+\partial_{z}^{\varphi} v=\frac{1+\left|\nabla_{y} \varphi\right|^{2}}{\partial_{z} \varphi} \partial_{z} v-\partial_{1} \varphi \partial_{1} v-\partial_{2} \varphi \partial_{2} v
$$

and

$$
\left(\nabla^{\varphi} v\right)^{t} \mathbf{N}=\left(\partial_{1}^{\varphi} v \cdot \mathbf{N}, \partial_{2}^{\varphi} v \cdot \mathbf{N}, \partial_{z}^{\varphi} v \cdot \mathbf{N}\right)^{t}=\left(\partial_{1} v \cdot \mathbf{N}, \partial_{2} v \cdot \mathbf{N}, 0\right)^{t}+\frac{1}{\partial_{z} \varphi}\left(\partial_{z} v \cdot \mathbf{N}\right) \mathbf{N}
$$

One can obtain immediately that

$$
\begin{aligned}
\Pi\left(\partial_{z} v\right) & =\frac{\partial_{z} \varphi}{1+\left|\nabla_{y} \varphi\right|^{2}}\left(\Pi\left(\nabla^{\varphi} v \mathbf{N}\right)+\Pi\left(\partial_{1} \varphi \partial_{1} v+\partial_{2} \varphi \partial_{2} v\right)\right) \\
& =\frac{\partial_{z} \varphi}{1+\left|\nabla_{y} \varphi\right|^{2}}\left(2 \Pi\left(S^{\varphi} v \mathbf{N}\right)-\Pi\left(\left(\nabla^{\varphi} v\right)^{t} \mathbf{N}\right)+\Pi\left(\partial_{1} \varphi \partial_{1} v+\partial_{2} \varphi \partial_{2} v\right)\right) \\
& =\frac{\partial_{z} \varphi}{1+\left|\nabla_{y} \varphi\right|^{2}}\left(2 \Pi\left(S^{\varphi} v \mathbf{N}\right)-\Pi\left\{\left(\partial_{1} v \cdot \mathbf{N}, \partial_{2} v \cdot \mathbf{N}, 0\right)^{t}\right\}+\Pi\left(\partial_{1} \varphi \partial_{1} v+\partial_{2} \varphi \partial_{2} v\right)\right) .
\end{aligned}
$$

As a consequence of $\partial_{z} v$ given in (4.4.3) and (4.4.6), one have the following estimates for $\partial_{z} v$ : 
Lemma 4.12 For any $k \in \mathbb{N}$, it holds that

$$
\int_{0}^{t}\left\|\partial_{z} v\right\|_{\mathcal{H}^{k}}^{2} \leq \Lambda_{0} \int_{0}^{t}\left\|\left(d i v^{\varphi} v, S_{\mathbf{n}}\right)\right\|_{\mathcal{H}^{k}}^{2}+\left\|V^{k+1}\right\|^{2} d \tau+\Lambda_{\infty, t} \int_{0}^{t}\left|\mathcal{Z}^{k} h\right|_{\frac{1}{2}}^{2} d \tau
$$

where $S_{\mathbf{n}}:=\Pi\left(S^{\varphi} v \mathbf{N}\right)$.

Furthermore, it follows from the expression of $\partial_{z z} v$ and (4.3.30) that

Lemma 4.13 For any $m \geq 1$, it holds that

$$
\varepsilon \int_{0}^{t}\left\|\nabla^{2} v(\tau)\right\|_{\mathcal{H}^{m-2}}^{2} d \tau \leq \Lambda_{0} \varepsilon \int_{0}^{t}\left\|\nabla^{\varphi} \mathcal{Z}^{m-2} S_{\mathbf{n}}(\tau)\right\|^{2} d \tau+\Lambda_{\infty}(t) \int_{0}^{t} Y_{m}(\tau) d \tau,
$$

and

$$
\begin{gathered}
\varepsilon^{2} \int_{0}^{t}\left\|\nabla^{2} v(\tau)\right\|_{\mathcal{H}^{m-1}}^{2} d \tau \leq \Lambda_{0} \varepsilon^{2} \int_{0}^{t}\left\|\left(\nabla^{\varphi} \mathcal{Z}^{m-1} d i v^{\varphi} v, \nabla^{\varphi} \mathcal{Z}^{m-1} S_{\mathbf{n}}, \nabla V^{m}\right)(\tau)\right\|^{2} d \tau \\
+\Lambda_{\infty}(t) \int_{0}^{t} Y_{m}(\tau)+\varepsilon\left|\mathcal{Z}^{m-1} \nabla_{y} h(\tau)\right|_{\frac{1}{2}}^{2} d \tau
\end{gathered}
$$

The key is to estimate $S_{\mathbf{n}}$. Now, we follow the argument in 32 for the convention-diffusion equation solved by $S_{\mathbf{n}}$ to derive the estimates of $\varepsilon \int_{0}^{t}\left\|\nabla^{\varphi} \mathcal{Z}^{m-2} S_{\mathbf{n}}(\tau)\right\|^{2} d \tau$ and $\varepsilon^{2} \int_{0}^{t}\left\|\nabla^{\varphi} \mathcal{Z}^{m-1} S_{\mathbf{n}}(\tau)\right\|^{2} d \tau$. The only difference is that $\operatorname{div}^{\varphi} v$ will be involved but has been estimated in the last subsection. It should be noted that $S_{\mathbf{n}}$ satisfies the homogeneous Dirichlet boundary condition

$$
S_{\mathbf{n}}=0, \quad \text { on } \quad z=0 .
$$

which follows from (1.33) and will be important for the following analysis.

Now, we start with the estimate of $S_{\mathbf{n}}$ in $\mathcal{H}^{m-2}$ which bound $\varepsilon \int_{0}^{t}\left\|\nabla^{\varphi} \mathcal{Z}^{m-2} S_{\mathbf{n}}(\tau)\right\|^{2} d \tau$.

Lemma 4.14 For any $t \in\left[0, T^{\varepsilon}\right]$ and $m \geq 2$, it holds that

$$
\begin{aligned}
& \left\|S_{\mathbf{n}}(t)\right\|_{\mathcal{H}^{m-2}}^{2}+\varepsilon \int_{0}^{t}\left\|\nabla^{\varphi} \mathcal{Z}^{m-2} S_{\mathbf{n}}(\tau)\right\|^{2} d \tau \\
& \leq \Lambda_{0}\left\|\mathcal{Z}^{\alpha} S_{\mathbf{n}}(0)\right\|^{2}+\delta \Lambda_{0} \varepsilon^{2} \int_{0}^{t}\left\|\left(\nabla^{\varphi} \mathcal{Z}^{m-1} \operatorname{div}^{\varphi} v, \nabla^{\varphi} \mathcal{Z}^{m-1} S_{\mathbf{n}}, \nabla^{\varphi} V^{m}\right)(\tau)\right\|^{2} d \tau \\
& \quad+C_{\delta} \Lambda\left(\frac{1}{c_{0}}, \mathcal{Q}(t)+\varepsilon\left\|\nabla^{\varphi} \operatorname{div}^{\varphi} v\right\|_{\mathcal{W}_{t}^{1, \infty}}^{2}\right) \int_{0}^{t} Y_{m}(\tau)+\varepsilon\left|\mathcal{Z}^{m} h\right|_{\frac{1}{2}}^{2} d \tau
\end{aligned}
$$

Proof. We first derive the equations satisfied by $S_{\mathbf{n}}$. Applying $\nabla^{\varphi}$ to $(1.31)_{2}$ gives that $\varrho \partial_{t}^{\varphi} \nabla^{\varphi} v+\varrho\left(v \cdot \nabla^{\varphi}\right) \cdot \nabla^{\varphi} v-\mu \varepsilon \Delta^{\varphi}\left(\nabla^{\varphi} v\right)=(\mu+\lambda) \varepsilon\left(\nabla^{\varphi}\right)^{2} \operatorname{div}^{\varphi} v-\left(\nabla^{\varphi}\right)^{2} p-\nabla^{\varphi} \varrho \otimes \partial_{t}^{\varphi} v-\nabla^{\varphi} v \nabla^{\varphi}(\varrho v)$, where $\left(\nabla^{\varphi}\right)^{2} f$ denotes the Hessian matrix of $f$. Thus,

$$
\begin{aligned}
& \varrho \partial_{t}^{\varphi}\left(S^{\varphi} v\right)+\varrho\left(v \cdot \nabla^{\varphi}\right)\left(S^{\varphi} v\right)-\mu \varepsilon \Delta^{\varphi}\left(S^{\varphi} v\right)=(\mu+\lambda) \varepsilon\left(\nabla^{\varphi}\right)^{2} \operatorname{div}^{\varphi} v-\left(\nabla^{\varphi}\right)^{2} p \\
& -\frac{1}{2}\left(\nabla^{\varphi} \varrho \otimes \partial_{t}^{\varphi} v+\partial_{t}^{\varphi} v \otimes \nabla^{\varphi} \varrho\right)-\frac{1}{2}\left(\nabla^{\varphi} v \nabla^{\varphi}(\varrho v)+\left(\nabla^{\varphi} v \nabla^{\varphi}(\varrho v)\right)^{t}\right) .
\end{aligned}
$$

Therefore, $S_{\mathbf{n}}$ satisfies

$$
\varrho \partial_{t}^{\varphi} S_{\mathbf{n}}+\varrho\left(v \cdot \nabla^{\varphi}\right) S_{\mathbf{n}}-\mu \varepsilon \Delta^{\varphi} S_{\mathbf{n}}=F,
$$

where $F$ is given by

$$
F=F_{1}+F_{2}+F_{3}
$$

with

$$
\begin{aligned}
F_{1}= & \varrho\left(\partial_{t}^{\varphi} \Pi+v \cdot \nabla^{\varphi} \Pi\right)\left(S^{\varphi} v \mathbf{N}\right)+\varrho \Pi\left(S^{\varphi} v\left(\partial_{t}^{\varphi} \mathbf{N}+v \cdot \nabla^{\varphi} \mathbf{N}\right)\right) \\
& -\frac{1}{2} \Pi\left\{\left(\nabla^{\varphi} \varrho \otimes \partial_{t}^{\varphi} v+\partial_{t}^{\varphi} v \otimes \nabla^{\varphi} \varrho\right) \mathbf{N}\right\}-\frac{1}{2} \Pi\left\{\left(\nabla^{\varphi} v \nabla^{\varphi}(\varrho v)+\left(\nabla^{\varphi} v \nabla^{\varphi}(\varrho v)\right)^{t}\right) \mathbf{N}\right\}, \\
F_{2}= & -\Pi\left\{\left(\nabla_{y}, 0\right)^{t}\left(\nabla^{\varphi} p \cdot \mathbf{N}\right)+\left(\nabla^{\varphi} \mathbf{N}\right)^{t} \nabla^{\varphi} p\right\}, \\
F_{3}= & (\mu+\lambda) \varepsilon \Pi\left\{\left(\nabla_{y}, 0\right)^{t}\left(\nabla^{\varphi} \operatorname{div}^{\varphi} v \cdot \mathbf{N}\right)-\left(\nabla^{\varphi} N\right)^{t} \nabla^{\varphi} \operatorname{div}^{\varphi} v\right\} \\
& -\mu \varepsilon\left(\Delta^{\varphi} \Pi\right)\left(S^{\varphi} v \mathbf{N}\right)-2 \mu \varepsilon \partial_{i}^{\varphi} \Pi \partial_{i}^{\varphi}\left(S^{\varphi} v \mathbf{N}\right)-\mu \varepsilon \Pi\left\{S^{\varphi} v \Delta^{\varphi} \mathbf{N}+2 \partial_{i}^{\varphi} S^{\varphi} v \partial_{i}^{\varphi} \mathbf{N}\right\} .
\end{aligned}
$$


where the summation convention has been used in the above expressions. It follows from Lemma 2.1. Lemma 2.3 and (4.3.31) that

$$
\begin{aligned}
\int_{0}^{t}\left\|F_{1}\right\|_{\mathcal{H}^{m-2}}^{2} d \tau \leq & \Lambda_{\infty}(t) \int_{0}^{t}\|(\nabla v, \nabla p)(\tau)\|_{\mathcal{H}^{m-2}}^{2}+\|(p, v)\|_{\mathcal{H}^{m-1}}^{2}+\left|\mathcal{Z}^{m-1} h\right|_{\frac{1}{2}}^{2} d \tau \\
\int_{0}^{t}\left\|F_{2}\right\|_{\mathcal{H}^{m-2}}^{2} d \tau \leq & \Lambda_{\infty}(t) \int_{0}^{t}\|\nabla p\|_{\mathcal{H}^{m-1}}^{2}+\left|\mathcal{Z}^{m-1} h\right|_{\frac{1}{2}}^{2} d \tau, \\
\int_{0}^{t}\left\|F_{3}\right\|_{\mathcal{H}^{m-2}}^{2} d \tau \leq & \Lambda_{0} \int_{0}^{t}\|v\|_{\mathcal{H}^{m-2}}^{2}+\varepsilon\left\|\nabla^{2} v\right\|_{\mathcal{H}^{m-2}}^{2}+\varepsilon^{2}\left\|\nabla^{2} v\right\|_{\mathcal{H}^{m-1}}^{2} d \tau \\
& +\Lambda\left(\frac{1}{c_{0}}, \mathcal{Q}(t)+\varepsilon\left\|\nabla \operatorname{div}^{\varphi} v\right\|_{\mathcal{W}_{t}^{1, \infty}}^{2}\right) \int_{0}^{t}\left|\mathcal{Z}^{m-1} h\right|_{\frac{1}{2}}^{2}+\varepsilon\left|\mathcal{Z}^{m} h\right|_{\frac{1}{2}}^{2} d \tau .
\end{aligned}
$$

The lemma is proved by induction. For the case $m=2$, multiplying (4.4.12) by $S_{\mathbf{n}}$, and then using Lemma 2.9 and the boundary condition (4.4.6), one gets that

$$
\int_{\mathcal{S}} \frac{1}{2} \varrho(t)\left|S_{\mathbf{n}}(t)\right|^{2} d \mathcal{V}_{t}+\mu \varepsilon \int_{0}^{t} \int_{\mathcal{S}}\left|\nabla^{\varphi} S_{\mathbf{n}}\right|^{2} d \mathcal{V}_{\tau} d \tau=\int_{\mathcal{S}} \frac{1}{2} \varrho_{0}\left|S_{\mathbf{n}}(0)\right|^{2} d \mathcal{V}_{0}+\int_{0}^{t} \int_{\mathcal{S}} F \cdot S_{\mathbf{n}} d \mathcal{V}_{\tau} d \tau
$$

It follows from (4.4.17)-(4.4.19) with $m=2$ that

$$
\begin{aligned}
& \int_{0}^{t} \int_{\mathcal{S}} F \cdot S_{\mathbf{n}} d \mathcal{V}_{\tau} d \tau \leq\left(\int_{0}^{t}\left\|\left(F_{1}, F_{2}, F_{3}\right)\right\|^{2} d \tau\right)^{\frac{1}{2}}\left(\int_{0}^{t}\left\|S_{\mathbf{n}}\right\|^{2} d \tau\right)^{\frac{1}{2}} \\
& \leq \int_{0}^{t}\|\nabla p\|_{\mathcal{H}^{1}}^{2}+\varepsilon^{2}\left\|\nabla \operatorname{div}^{\varphi} v\right\|_{\mathcal{H}^{1}}^{2}+\varepsilon^{2}\left\|\nabla^{2} v\right\|_{\mathcal{H}^{1}}^{2} d \tau \\
& \quad+\Lambda\left(\frac{1}{c_{0}}, \mathcal{Q}(t)+\left\|\nabla^{\varphi} \operatorname{div}^{\varphi} v\right\|_{\infty, t}^{2}\right) \int_{0}^{t}\|v\|_{\mathcal{H}^{2}}^{2}+\|(p, \nabla v)\|_{\mathcal{H}^{1}}^{2}+|\mathcal{Z} h|_{\frac{1}{2}}^{2}+\varepsilon\left|\mathcal{Z}^{2} h\right|_{\frac{1}{2}}^{2} \\
& \leq \Lambda\left(\frac{1}{c_{0}}, \mathcal{Q}(t)+\left\|\nabla^{\varphi} \operatorname{div}^{\varphi} v\right\|_{\infty, t}^{2}\right) \int_{0}^{t} \Lambda\left(Y_{m}(\tau)\right)+\varepsilon\left|\mathcal{Z}^{2} h\right|_{\frac{1}{2}}^{2} d \tau,
\end{aligned}
$$

where (4.3.31) has been used in the last inequality. Substituting (4.4.21) into (4.4.20), one gets from (4.3.30) and (4.4.9) that

$$
\begin{aligned}
& \int_{\mathcal{S}} \frac{1}{2} \varrho(t)\left|S_{\mathbf{n}}(t)\right|^{2} d \mathcal{V}_{t}+\mu \varepsilon \int_{0}^{t} \int\left|\nabla^{\varphi} S_{\mathbf{n}}\right|^{2} d \mathcal{V}_{\tau} d \tau \\
& \leq \int_{\mathcal{S}} \frac{1}{2} \varrho_{0}\left|S_{\mathbf{n}}(0)\right|^{2} d \mathcal{V}_{0}+\Lambda_{0} \varepsilon^{2} \int_{0}^{t}\left\|\nabla^{\varphi} \mathcal{Z} \operatorname{div}^{\varphi} v, \nabla^{\varphi} \mathcal{Z} S_{\mathbf{n}}, \nabla V^{1}\right\| d \tau \\
& \quad+\Lambda\left(\frac{1}{c_{0}}, \mathcal{Q}(t)+\left\|\nabla^{\varphi} \operatorname{div}^{\varphi} v\right\|_{\infty, t}^{2}\right) \int_{0}^{t} \Lambda\left(Y_{m}(\tau)\right)+\varepsilon\left|\mathcal{Z}^{2} h\right|_{\frac{1}{2}}^{2} d \tau .
\end{aligned}
$$

Assume that (4.4.11) has been proved for $k \leq m-3$, we shall prove it for $k=m-2$. First, applying $\mathcal{Z}^{\alpha}$, with $|\alpha|=m-2$, to (4.4.12) yields that

$$
\varrho \partial_{t}^{\varphi} \mathcal{Z}^{\alpha} S_{\mathbf{n}}+\varrho v \cdot \nabla^{\varphi} \mathcal{Z}^{\alpha} S_{\mathbf{n}}-\mu \varepsilon \Delta^{\varphi} \mathcal{Z}^{\alpha} S_{\mathbf{n}}=\mathcal{Z}^{\alpha} F+C_{S},
$$

with $C_{S}=C_{S}^{1}+C_{S}^{2}$ given by

$$
C_{S}^{1} \doteq-\left[\mathcal{Z}^{\alpha}, \varrho\right] \partial_{t} S_{\mathbf{n}}-\left[\mathcal{Z}^{\alpha}, \varrho v_{y}\right] \nabla_{y} S_{\mathbf{n}}-\left[\mathcal{Z}^{\alpha}, \varrho V_{z}\right] \partial_{z} S_{\mathbf{n}}-\varrho V_{z}\left[\mathcal{Z}^{\alpha}, \partial_{z}\right] S_{\mathbf{n}} \doteq \sum_{i=1}^{4} C_{S}^{1, i},
$$

and

$$
C_{S}^{2} \doteq \mu \varepsilon\left[\mathcal{Z}^{\alpha}, \Delta^{\varphi}\right] S_{\mathbf{n}}
$$

Multiplying (4.4.23) by $\mathcal{Z}^{\alpha} S_{\mathbf{n}}$ with $|\alpha|=m-2$ and integrating give that

$$
\begin{aligned}
& \int_{\mathcal{S}} \frac{1}{2} \varrho(t)\left|\mathcal{Z}^{\alpha} S_{\mathbf{n}}(t)\right|^{2} d \mathcal{V}_{t}+\mu \varepsilon \int_{0}^{t} \int_{\mathcal{S}}\left|\nabla^{\varphi} \mathcal{Z}^{\alpha} S_{\mathbf{n}}\right|^{2} d \mathcal{V}_{\tau} d \tau \\
& =\int_{\mathcal{S}} \frac{1}{2} \varrho_{0}\left|\mathcal{Z}^{\alpha} S_{\mathbf{n}}(0)\right|^{2} d \mathcal{V}_{0}+\int_{0}^{t} \int_{\mathcal{S}}\left|\mathcal{Z}^{\alpha} F \cdot \mathcal{Z}^{\alpha} S_{\mathbf{n}}\right| d \mathcal{V}_{\tau} d \tau+\int_{0}^{t} \int_{\mathcal{S}} C_{S} \cdot \mathcal{Z}^{\alpha} S_{\mathbf{n}} d \mathcal{V}_{\tau} d \tau
\end{aligned}
$$


As a consequence of (4.4.17)-(4.4.19), (4.3.42), (4.4.8) and (4.4.9), we obtain that

$$
\begin{aligned}
& \int_{0}^{t} \int_{\mathcal{S}}\left|\mathcal{Z}^{\alpha} F \cdot \mathcal{Z}^{\alpha} S_{\mathbf{n}}\right| d \mathcal{V}_{\tau} d \tau \leq\left(\int_{0}^{t}\left\|\left(F_{1}, F_{2}, F_{3}\right)\right\|_{\mathcal{H}^{m-2}}^{2} d \tau\right)^{\frac{1}{2}}\left(\int_{0}^{t}\left\|S_{\mathbf{n}}\right\|_{\mathcal{H}^{m-2}}^{2} d \tau\right)^{\frac{1}{2}} \\
& \leq \delta \int_{0}^{t}\|\nabla p\|_{\mathcal{H}^{m-1}}^{2}+\varepsilon\left\|\nabla^{2} v\right\|_{\mathcal{H}^{m-2}}^{2}+\varepsilon^{2}\left\|\nabla^{2} v\right\|_{\mathcal{H}^{m-1}}^{2} d \tau \\
& \quad+\Lambda\left(\frac{1}{c_{0}}, \mathcal{Q}(t)+\varepsilon\left\|\nabla \operatorname{div}^{\varphi} v\right\|_{\mathcal{W}_{t}^{1, \infty}}^{2}\right) \int_{0}^{t} Y^{m}(\tau)+\varepsilon\left|\mathcal{Z}^{m} h\right|_{\frac{1}{2}}^{2} d \tau . \\
& \leq \frac{\mu \varepsilon}{4} \int_{0}^{t}\left\|\nabla^{\varphi} \mathcal{Z}^{m-2} S_{\mathbf{n}}\right\|^{2} d \tau+\delta \Lambda_{0} \varepsilon^{2} \int_{0}^{t}\left\|\left(\nabla^{\varphi} \mathcal{Z}^{m-1} \operatorname{div}^{\varphi} v, \nabla^{\varphi} \mathcal{Z}^{m-1} S_{\mathbf{n}}, \nabla^{\varphi} V^{m}\right)(\tau)\right\|^{2} d \tau \\
& \quad+C_{\delta} \Lambda\left(\frac{1}{c_{0}}, \mathcal{Q}(t)+\varepsilon\left\|\nabla \operatorname{div}^{\varphi} v\right\|_{\mathcal{W}_{t}^{1, \infty}}^{2}\right) \int_{0}^{t} Y_{m}(\tau)+\varepsilon\left|\mathcal{Z}^{m} h(t)\right|_{\frac{1}{2}}^{2} d \tau .
\end{aligned}
$$

It remains to estimate the terms involving $C_{S}^{1}, C_{S}^{2}$. Similar to [32, integrating by parts and using the Hardy inequality, one gets that

$$
\int_{0}^{t} \int_{\mathcal{S}}\left|C_{S}^{1} \mathcal{Z}^{\alpha} S_{\mathbf{n}}\right| d \mathcal{V}_{t} d \tau \leq \int_{0}^{t} \Lambda\left(Y_{m}(\tau)\right) d \tau
$$

For the term involving $C_{S}^{2}$, we notice that $\Delta^{\varphi}$ can be rewritten as

$$
\Delta^{\varphi} f=\frac{1}{\partial_{z} \varphi} \nabla \cdot(E \nabla f)
$$

with the matrix $E$ defined by

$$
E=\left(\begin{array}{ccc}
\partial_{z} \varphi & 0 & -\partial_{1} \varphi \\
0 & \partial_{z} \varphi & -\partial_{2} \varphi \\
-\partial_{1} \varphi & -\partial_{2} \varphi & \frac{1+\left|\nabla_{y} \varphi\right|^{2}}{\partial_{z} \varphi}
\end{array}\right)
$$

This yields immediately that

$$
C_{S}^{2}=C_{S}^{2,1}+C_{S}^{2,2}+C_{S}^{2,3}
$$

with

$$
C_{S}^{2,1}=\mu \varepsilon\left[\mathcal{Z}^{\alpha}, \frac{1}{\partial_{z} \varphi}\right] \nabla \cdot\left(E \nabla S_{\mathbf{n}}\right), \quad C_{S}^{2,2}=\mu \varepsilon \frac{1}{\partial_{z} \varphi}\left[\mathcal{Z}^{\alpha}, \nabla \cdot\right]\left(E \nabla S_{\mathbf{n}}\right), \quad C_{S}^{2,3}=\mu \varepsilon \frac{1}{\partial_{z} \varphi} \nabla \cdot\left[\mathcal{Z}^{\alpha}, E \nabla\right] S_{\mathbf{n}} .
$$

By similar arguments as [32, one obtains that

$$
\begin{aligned}
\left|\int_{0}^{t} \int_{\mathcal{S}} C_{S}^{2} \mathcal{Z}^{\alpha} S_{\mathbf{n}} d \mathcal{V}_{\tau} d \tau\right| \leq & \frac{\mu \varepsilon}{16} \int_{0}^{t} \int\left|\nabla \mathcal{Z}^{m-2} S_{\mathbf{n}}\right|^{2} d \mathcal{V}_{\tau}+\Lambda_{0} \varepsilon \int_{0}^{t} \int\left|\nabla \mathcal{Z}^{m-3} S_{\mathbf{n}}\right|^{2} d \mathcal{V}_{\tau} d \tau \\
& +\Lambda_{\infty}(t) \int_{0}^{t} Y_{m}(\tau) d \tau
\end{aligned}
$$

Plugging (4.4.27), (4.4.28) and (4.4.31) into (4.4.26), we obtain that

$$
\begin{aligned}
& \int_{\mathcal{S}} \frac{1}{2} \varrho(t)\left|\mathcal{Z}^{\alpha} S_{\mathbf{n}}(t)\right|^{2} d \mathcal{V}_{t}+\mu \varepsilon \int_{0}^{t} \int_{\mathcal{S}}\left|\nabla^{\varphi} \mathcal{Z}^{\alpha} S_{\mathbf{n}}\right|^{2} d \mathcal{V}_{\tau} d \tau \\
& \leq \int_{\mathcal{S}} \frac{1}{2} \varrho_{0}\left|\mathcal{Z}^{\alpha} S_{\mathbf{n}}(0)\right|^{2} d \mathcal{V}_{0}+\Lambda_{0} \varepsilon \int_{0}^{t} \int\left|\nabla \mathcal{Z}^{m-3} S_{\mathbf{n}}\right|^{2} d \mathcal{V}_{\tau} d \tau+\Lambda_{\infty}(t) \int_{0}^{t} \Lambda\left(Y_{m}(\tau)\right)+\varepsilon\left|\mathcal{Z}^{m} h\right|_{\frac{1}{2}}^{2} d \tau
\end{aligned}
$$

Thus, the lemma is proved by (2.10) and the induction assumption to control $\Lambda_{0} \varepsilon \int_{0}^{t}\left\|\nabla^{\varphi} \mathcal{Z}^{m-3} S_{\mathbf{n}}\right\|^{2} d \tau$.

The following lemma give the estimate of $\varepsilon^{2} \int_{0}^{t}\left\|\nabla^{\varphi} \mathcal{Z}^{m-1} S_{\mathbf{n}}\right\|^{2} d \tau$. 
Lemma 4.15 For any $t \in\left[0, T^{\varepsilon}\right]$ and $m \geq 1$, it holds that

$$
\begin{aligned}
\varepsilon\left\|S_{\mathbf{n}}(t)\right\|_{\mathcal{H}^{m-1}}^{2}+\varepsilon^{2} \int_{0}^{t}\left\|\nabla^{\varphi} \mathcal{Z}^{m-1} S_{\mathbf{n}}\right\|^{2} d \tau \\
\leq \frac{1}{\delta_{1}} \Lambda\left(\frac{1}{c_{0}}, Y_{m}(0)\right)+\Lambda_{0} \delta_{1} \varepsilon^{2} \int_{0}^{t} \int\left|\nabla^{\varphi} \mathcal{Z}^{m-1} d i v^{\varphi} v\right|^{2} d \mathcal{V}_{\tau} d \tau \\
\quad+C_{\delta_{1}} \Lambda_{\infty}(t) \int_{0}^{t} \Lambda\left(Y_{m}(\tau)\right)+\varepsilon\left|\mathcal{Z}^{m} h\right|_{\frac{1}{2}}^{2}+\|\nabla v\|_{\mathcal{H}^{m-1}}^{2} d \tau
\end{aligned}
$$

where $\delta_{1}>0$ and $\delta>0$ will be chosen later.

Proof. Multiplying (4.4.23) by $\varepsilon \mathcal{Z}^{\alpha} S_{\mathbf{n}}$ with $|\alpha|=m-1$ and integrating give that

$$
\begin{aligned}
& \int_{\mathcal{S}} \frac{\varepsilon}{2} \varrho(t)\left|\mathcal{Z}^{\alpha} S_{\mathbf{n}}(t)\right|^{2} d \mathcal{V}_{t}+\mu \varepsilon^{2} \int_{0}^{t} \int_{\mathcal{S}}\left|\nabla^{\varphi} \mathcal{Z}^{\alpha} S_{\mathbf{n}}\right|^{2} d \mathcal{V}_{\tau} d \tau \\
& =\int_{\mathcal{S}} \frac{\varepsilon}{2} \varrho_{0}\left|\mathcal{Z}^{\alpha} S_{\mathbf{n}}(0)\right|^{2} d \mathcal{V}_{0}+\varepsilon\left|\int_{0}^{t} \int_{\mathcal{S}} \mathcal{Z}^{\alpha} F \cdot \mathcal{Z}^{\alpha} S_{\mathbf{n}} d \mathcal{V}_{\tau} d \tau\right|+\varepsilon\left|\int_{0}^{t} \int_{\mathcal{S}} C_{S} \cdot \mathcal{Z}^{\alpha} S_{\mathbf{n}} d \mathcal{V}_{\tau} d \tau\right| .
\end{aligned}
$$

Similar to (4.4.17), it is easy to obtain that

$$
\int_{0}^{t}\left\|F_{1}\right\|_{\mathcal{H}^{m-1}}^{2} d \tau \leq \Lambda_{\infty}(t) \int_{0}^{t}\|(\nabla v, \nabla p)\|_{\mathcal{H}^{m-1}}^{2}+\|(p, v)\|_{\mathcal{H}^{m}}^{2}+\left|\mathcal{Z}^{m} h\right|_{\frac{1}{2}}^{2} d \tau
$$

which implies that

$$
\varepsilon\left|\int_{0}^{t} \int_{\mathcal{S}} \mathcal{Z}^{\alpha} F_{1} \cdot \mathcal{Z}^{\alpha} S_{\mathbf{n}} d \mathcal{V}_{\tau} d \tau\right| \leq \Lambda_{\infty}(t) \int_{0}^{t} Y_{m}(\tau)+\varepsilon\left|\mathcal{Z}^{m} h\right|_{\frac{1}{2}}^{2} d \tau
$$

Using integration by parts, one can get that

$$
\begin{gathered}
\varepsilon\left|\int_{0}^{t} \int_{\mathcal{S}} \mathcal{Z}^{\alpha} F_{2} \cdot \mathcal{Z}^{\alpha} S_{\mathbf{n}} d \mathcal{V}_{\tau} d \tau\right| \leq \varepsilon \int_{0}^{t}\left\|\nabla V^{m}\right\|^{2} d \tau+\Lambda_{\infty}(t) \int_{0}^{t} Y_{m}(\tau)+\varepsilon\left|\mathcal{Z}^{m} h\right|_{\frac{1}{2}}^{2} d \tau \\
\varepsilon\left|\int_{0}^{t} \int_{\mathcal{S}} \mathcal{Z}^{\alpha} F_{3} \cdot \mathcal{Z}^{\alpha} S_{\mathbf{n}} d \mathcal{V}_{\tau} d \tau\right| \\
\leq \frac{\mu \varepsilon^{2}}{16} \int_{0}^{t} \int\left|\nabla^{\varphi} \mathcal{Z}^{\alpha} S_{\mathbf{n}}\right|^{2} d \mathcal{V}_{\tau} d \tau+\Lambda_{0} \delta_{1} \varepsilon^{2} \int_{0}^{t} \int\left|\nabla^{\varphi} \mathcal{Z}^{m-1} \operatorname{div}^{\varphi} v\right|^{2} d \mathcal{V}_{\tau} d \tau \\
\quad+\frac{\Lambda_{0} \varepsilon^{2}}{\delta_{1}} \int_{0}^{t}\left\|\nabla V^{m}\right\|^{2} d \tau+C_{\delta_{1}} \Lambda_{\infty}(t) \int_{0}^{t} \Lambda\left(Y_{m}(\tau)\right)+\varepsilon\left|\mathcal{Z}^{m} h\right|_{\frac{1}{2}}^{2} d \tau
\end{gathered}
$$

and

$$
\begin{aligned}
& \varepsilon\left|\int_{0}^{t} \int_{\mathcal{S}} C_{S}^{1} \mathcal{Z}^{\alpha} S_{\mathbf{n}} d \mathcal{V}_{\tau} d \tau\right|+\varepsilon\left|\int_{0}^{t} \int_{\mathcal{S}} C_{S}^{2} \mathcal{Z}^{\alpha} S_{\mathbf{n}} d \mathcal{V}_{\tau} d \tau\right| \\
& \leq \frac{\mu \varepsilon^{2}}{16} \int_{0}^{t} \int\left|\nabla^{\varphi} \mathcal{Z}^{\alpha} S_{\mathbf{n}}\right|^{2} d \mathcal{V}_{\tau} d \tau+\Lambda_{\infty}(t) \int_{0}^{t} \Lambda\left(Y_{m}(\tau)\right)+\varepsilon\left|\mathcal{Z}^{m} h\right|_{\frac{1}{2}}^{2} d \tau
\end{aligned}
$$

Substituting (4.4.34)-4.4.37) into (4.4.33), we prove (4.4.33). Therefore, the proof of this lemma is completed.

Then, suitable choice of $\delta$ and $\delta_{1}$ yield the following proposition

Proposition 4.16 For any $t \in\left[0, T^{\varepsilon}\right], m \geq 5$, it holds that

$$
\begin{aligned}
& \left\|\left(V^{m}, Q^{m}\right)(t)\right\|^{2}+\left|\left(h, \sqrt{\sigma} \nabla_{y} h\right)(t)\right|_{\mathcal{H}^{m}}^{2}+\|(\nabla p, \nabla v)(t)\|_{\mathcal{H}^{m-2}}^{2}+\varepsilon\left\|\left(d i v^{\varphi} v, \nabla^{\varphi} p, S_{\mathbf{n}}\right)(t)\right\|_{\mathcal{H}^{m-1}}^{2} \\
& \quad+\varepsilon\left|\mathcal{Z}^{m} h\right|_{\frac{1}{2}}^{2}+\int_{0}^{t}\|\nabla p\|_{\mathcal{H}^{m-1}}^{2} d \tau+\varepsilon \int_{0}^{t}\|\nabla v\|_{\mathcal{H}^{m}}^{2} d \tau+\int_{0}^{t} \varepsilon\left\|\nabla^{2} v\right\|_{\mathcal{H}^{m-2}}^{2}+\varepsilon^{2}\left\|\nabla^{2} v\right\|_{\mathcal{H}^{m-1}}^{2} d \tau \\
& \leq \Lambda\left(\frac{1}{c_{0}}, Y_{m}(0)\right)+\Lambda_{\infty}(t)\left(1+\left\|\nabla^{\varphi} d i v^{\varphi} v\right\|_{\infty, t}^{2}\right) \int_{0}^{t} \Lambda\left(Y_{m}(\tau)\right)+\varepsilon\left|\mathcal{Z}^{m} h\right|_{\frac{1}{2}}^{2}+\|\nabla v\|_{\mathcal{H}^{m-1}}^{2} d \tau
\end{aligned}
$$


Proof. Combining Propositions 4.4 4.5 Lemmas $4.13,4.14$ and 4.15 one can obtain that

$$
\begin{aligned}
& \left\|\left(V^{m}, Q^{m}\right)(t)\right\|^{2}+\left|\left(h, \sqrt{\sigma} \nabla_{y} h\right)(t)\right|_{\mathcal{H}^{m}}^{2}+\|\nabla v(t)\|_{\mathcal{H}^{m-2}}^{2}+\varepsilon\left\|\left(\operatorname{div}^{\varphi} v, \nabla^{\varphi} p, S_{\mathbf{n}}\right)(t)\right\|_{\mathcal{H}^{m-1}}^{2} \\
& \quad+\int_{0}^{t} \varepsilon\left\|\nabla V^{m}(\tau)\right\|^{2}+\varepsilon\left\|\nabla^{2} v(\tau)\right\|_{\mathcal{H}^{m-2}}^{2}+\varepsilon^{2}\left\|\nabla^{2} v(\tau)\right\|_{\mathcal{H}^{m-1}}^{2} d \tau \\
& \leq \frac{1}{\delta_{1}} \Lambda\left(\frac{1}{c_{0}}, Y_{m}(0)\right)+\Lambda_{0}\left(\delta_{1}+\Lambda_{0} \frac{\delta}{\delta_{1}}\right) \varepsilon^{2} \int_{0}^{t}\left\|\nabla^{2} v(\tau)\right\|_{\mathcal{H}^{m-1}}^{2} d \tau+\left(\delta_{1}+\Lambda_{0} \frac{\delta}{\delta_{1}}\right) \varepsilon \int_{0}^{t}\left\|\nabla V^{m}\right\| d \tau \\
& \quad+C_{\delta} \Lambda_{\infty}(t)\left(1+\left\|\nabla^{\varphi} \operatorname{div}^{\varphi} v\right\|_{L_{t, x}^{\infty}}^{2}\right) \int_{0}^{t} \Lambda\left(Y_{m}(\tau)\right)+\varepsilon\left|\mathcal{Z}^{m} h\right|_{\frac{1}{2}}^{2}+\|\nabla v\|_{\mathcal{H}^{m-1}}^{2} d \tau .
\end{aligned}
$$

Choosing $\delta_{1}>0$ to be small such that $\delta_{1} \leq \frac{1}{4 \Lambda_{0}}$, and $\delta=\frac{\delta_{1}^{2}}{\Lambda_{0}}$ yields that

$$
\begin{aligned}
& \left\|\left(V^{m}, Q^{m}\right)(t)\right\|^{2}+\left|\left(h, \sqrt{\sigma} \nabla_{y} h\right)(t)\right|_{\mathcal{H}^{m}}^{2}+\|\nabla v(t)\|_{\mathcal{H}^{m-2}}^{2}+\varepsilon\left\|\left(\operatorname{div}^{\varphi} v, \nabla^{\varphi} p, S_{\mathbf{n}}\right)(t)\right\|_{\mathcal{H}^{m-1}}^{2} \\
& +\int_{0}^{t} \varepsilon\left\|\nabla^{\varphi} V^{m}(\tau)\right\|^{2}+\varepsilon\left\|\nabla^{2} v(\tau)\right\|_{\mathcal{H}^{m-2}}^{2}+\varepsilon^{2}\left\|\nabla^{2} v(\tau)\right\|_{\mathcal{H}^{m-1}}^{2} d \tau \\
& \leq \Lambda\left(\frac{1}{c_{0}}, Y_{m}(0)\right)+\Lambda_{\infty}(t)\left(1+\left\|\nabla^{\varphi} \operatorname{div}^{\varphi} v\right\|_{L_{t, x}^{\infty}}^{2}\right) \int_{0}^{t} \Lambda\left(Y_{m}(\tau)\right)+\varepsilon\left|\mathcal{Z}^{m} h\right|_{\frac{1}{2}}^{2}+\|\nabla v\|_{\mathcal{H}^{m-1}}^{2} d \tau .
\end{aligned}
$$

This, together with (4.3.2) and Lemma 2.8, yields immediately (4.4.38). Thus, the proof of this proposition is completed.

\subsection{Normal derivative estimates: Part II}

Now we start to estimate $\int_{0}^{t}\|\nabla v\|_{\mathcal{H}^{m-1}}^{2} d \tau$. It follows from Lemmas 4.10 and 4.12 that one needs only to bound $\left\|S_{\mathbf{n}}\right\|_{\mathcal{H}^{m-1}}$. However, this seems difficult since $\left\|\nabla^{\varphi} p\right\|_{\mathcal{H}^{m}}$ and $\left\|\nabla^{\varphi} \operatorname{div}^{\varphi} v\right\|_{\mathcal{H}^{m}}$, which appear in $F_{2}$ and $F_{3}$ in the equation (4.4.12) for $S_{\mathbf{n}}$, cannot be estimated uniformly in $\varepsilon$ (c.f. Lemma 4.15). As in [32], we use the vorticity instead of $S_{\mathbf{n}}$ to perform this estimate. Let us set

$$
\omega_{\mathbf{n}}:=\omega \times \mathbf{N}=\Pi(\omega \times \mathbf{N})
$$

where $\omega$ is the vorticity defined by $\omega=\nabla^{\varphi} \times v$. Since (4.4.5) yields that

$$
2 S_{\mathbf{n}}=\omega_{\mathbf{n}}+2 \Pi\left\{\left(\partial_{1} v \cdot \mathbf{N}, \partial_{2} v \cdot \mathbf{N}, 0\right)^{t}\right\},
$$

one can obtain from (4.4.10) that

$$
\left(\omega_{\mathbf{n}}\right)^{b}=-2 \Pi\left\{\left(\partial_{1} v \cdot \mathbf{N}, \partial_{2} v \cdot \mathbf{N}, 0\right)^{t}\right\} .
$$

which implies that

$$
\left(\mathcal{Z}^{\alpha} \omega \times \mathbf{N}\right)^{b}=-\left(\omega \times \mathcal{Z}^{\alpha} \mathbf{N}\right)^{b}-\left(\left[\mathcal{Z}^{\alpha}, \omega \times, \mathbf{N}\right]\right)^{b}-2 \mathcal{Z}^{\alpha}\left(\Pi\left\{\left(\partial_{1} v \cdot \mathbf{N}, \partial_{2} v \cdot \mathbf{N}, 0\right)^{t}\right\}\right) .
$$

Thus, one can obtain the sharp estimate that on the boundary, for $\alpha \leq m-1$,

$$
\begin{aligned}
\sqrt{\varepsilon} \int_{0}^{t}\left|\left(\mathcal{Z}^{\alpha} \omega \times \mathbf{N}\right)^{b}\right|^{2} d \tau & \leq \Lambda_{0} \sqrt{\varepsilon} \int_{0}^{t}\left|\omega^{b}\right|_{\mathcal{H}^{m-2}}^{2}+\left|v^{b}\right|_{\mathcal{H}^{m}}^{2} d \tau+\Lambda_{\infty}(t) \int_{0}^{t} \sqrt{\varepsilon}\left|\nabla_{y} h\right|_{\mathcal{H}^{m-1}}^{2} d \tau \\
& \leq \Lambda_{0} \varepsilon \int_{0}^{t}\left\|\nabla^{2} v\right\|_{\mathcal{H}^{m-2}}+\|\nabla v\|_{\mathcal{H}^{m}}^{2}+\Lambda_{\infty}(t) \int_{0}^{t} Y_{m}(\tau) d \tau .
\end{aligned}
$$

Hence, one can only expect to bound $\int_{0}^{t}\left\|\mathcal{Z}^{m-1} \omega \times \mathbf{N}\right\|^{4} d \tau$ by the similar argument in 32. Here and henceforth, $\left\|\mathcal{Z}^{m-1} \omega \times \mathbf{N}\right\|^{2}=\sum_{|\alpha| \leq m-1}\left\|\mathcal{Z}^{\alpha} \omega \times \mathbf{N}\right\|^{2}$. It should be emphasized that, one may not expect to bound directly $\mathcal{Z}^{m-1} \omega$ like [32, since it involves $\sqrt{\varepsilon} \int_{0}^{t}\left|\omega^{b}\right|_{\mathcal{H}^{m-1}}^{2} d \tau$ which seems impossible to be bounded due to the invalid of (1.62) in the compressible flows. However, as shown in (4.5.2), $\omega_{\mathbf{n}}$ involves only the tangential derivatives of $v$ so that one can follow the argument in 32] for $\omega_{\mathbf{n}}$ instead of $\omega$. Actually, we will bound $\mathcal{Z}^{m-1} \omega \times \mathbf{N}$ to avoid too much regularity of $h$ involving.

The main result of this subsection is the following proposition: 
Proposition 4.17 It holds that, for any $m \geq 6$ that

$$
\begin{aligned}
\left(\int_{0}^{t}\|\nabla v\|_{\mathcal{H}^{m-1}}^{4}\right)^{\frac{1}{2}} \leq & \Lambda_{0}\left\|\mathcal{Z}^{\alpha} \omega_{0}\right\|^{2}+\varepsilon \int_{0}^{t}\left\|\nabla V^{m}\right\|^{2}+\left\|\nabla^{\varphi} \mathcal{Z}^{m-2} d i v^{\varphi} v\right\|^{2}+\left\|\nabla^{\varphi} \mathcal{Z}^{m-2} S_{\mathbf{n}}\right\|^{2} d \tau \\
& +\Lambda(M(t)) \int_{0}^{t} \Lambda\left(Y_{m}(\tau)\right) d \tau+\Lambda_{\infty}(t) t^{\frac{1}{2}} \int_{0}^{t}\|\nabla p\|_{\mathcal{H}^{m-1}}^{2} d \tau
\end{aligned}
$$

where

$$
M(t):=\sup _{0 \leq \tau \leq t}\left(\Lambda\left(\frac{1}{c_{0}}, \mathcal{Q}(\tau)+\mathcal{Q}_{m}(\tau)+\varepsilon\left|\mathcal{Z}^{m} h\right|_{\frac{1}{2}}^{2}\right)+\varepsilon \int_{0}^{\tau}\left\|\nabla V^{6}\right\|^{2}+\left\|\nabla^{2} v\right\|_{\mathcal{H}^{4}}^{2} d s\right) .
$$

The proof of this proposition is a consequence of (4.5.10) and Proposition 4.18 below.

Since

$$
\mathcal{Z}^{\alpha} \omega_{\mathbf{n}}=\mathcal{Z}^{\alpha}(\omega \times \mathbf{N})=\mathcal{Z}^{\alpha} \omega \times \mathbf{N}-\left[\mathcal{Z}^{\alpha}, \mathbf{N} \times\right] \omega, \quad|\alpha| \leq m-1,
$$

and

$$
\begin{aligned}
\left\|-\left[\mathcal{Z}^{\alpha}, \mathbf{N} \times\right] \omega\right\|^{2} & \leq\|\omega\|_{\infty}^{2}\left\|\nabla_{y} \eta\right\|_{\mathcal{H}^{m-1}}^{2}+\|\omega\|_{\mathcal{H}^{1, \infty}}^{2}\left\|\nabla_{y} \eta\right\|_{\mathcal{H}^{m-2}}^{2}+\sum_{\substack{|\beta|=1 \\
\beta+\nu=\alpha}}^{|\beta|=m-3}\left\|\mathcal{Z}^{\beta} \mathbf{N} \times \mathcal{Z}^{\nu} \omega\right\|^{2} \\
& \leq \Lambda\left(\frac{1}{c_{0}}, \mathcal{Q}_{m}(t)+\mathcal{Q}(t)\right),
\end{aligned}
$$

we can obtain from (4.4.1) (4.4.3), (4.4.6) (4.3.44) and 4.5.2) that

$$
\begin{aligned}
\int_{0}^{t}\left\|\partial_{z} v\right\|_{\mathcal{H}^{m-1}}^{4} d \tau & \leq \Lambda_{0} \int_{0}^{t}\left\|\mathcal{Z}^{m-1} \omega \times \mathbf{N}\right\|^{4}+\left\|\operatorname{div}^{\varphi} v\right\|_{\mathcal{H}^{m-1}}^{4} d \tau+\Lambda_{\infty}(t) \int_{0}^{t} \Lambda\left(Y_{m}(\tau)\right) d \tau \\
& \leq \Lambda_{0} \int_{0}^{t}\left\|\mathcal{Z}^{m-1} \omega \times \mathbf{N}\right\|^{4} d \tau+\Lambda_{\infty}(t) \int_{0}^{t} \Lambda\left(Y_{m}(\tau)\right) d \tau
\end{aligned}
$$

So, it remains to control $\mathcal{Z}^{\alpha} \omega \times \mathbf{N}$, for $|\alpha| \leq m-1$.

Now we first derive the equation solved by $\mathcal{Z}^{\alpha} \omega \times \mathbf{N}$ as follows. Applying $\nabla^{\varphi} \times$ to $(1.31)_{2}$ yields that

$$
\varrho \partial_{t}^{\varphi} \omega+\varrho v \cdot \nabla^{\varphi} \omega-\mu \varepsilon \Delta^{\varphi} \omega=F_{1},
$$

where $F_{1}$

$$
F_{1}=-\nabla^{\varphi} \varrho \times \partial_{t}^{\varphi} v-\nabla^{\varphi} \varrho \times\left(\left(v \cdot \nabla^{\varphi}\right) v\right)+\varrho \omega \cdot \nabla^{\varphi} v-\varrho \operatorname{div}^{\varphi} v \omega .
$$

Applying $\mathcal{Z}^{\alpha}$, with $|\alpha|=m-1$, to (4.5.11) gives that

$$
\varrho \partial_{t} \mathcal{Z}^{\alpha} \omega+\varrho v_{y} \cdot \nabla_{y} \mathcal{Z}^{\alpha} \omega+\varrho V_{z} \partial_{z} \mathcal{Z}^{\alpha} \omega-\mu \varepsilon \Delta^{\varphi} \mathcal{Z}^{\alpha} \omega=\mathcal{Z}^{\alpha} F_{1}+F_{2},
$$

where

$$
F_{2}=\left[\mathcal{Z}^{\alpha}, \varrho\right] \partial_{t} \omega+\left[\mathcal{Z}^{\alpha}, \varrho v_{y}\right] \nabla_{y} \omega+\left[\mathcal{Z}^{\alpha}, \varrho V_{z}\right] \partial_{z} \omega+\varrho V_{z}\left[\mathcal{Z}^{\alpha}, \partial_{z}\right] \omega+\mu \varepsilon\left[\mathcal{Z}^{\alpha}, \Delta^{\varphi}\right] \omega .
$$

Multiply (4.5.13) to get that

$$
\varrho \partial_{t}\left(\mathcal{Z}^{\alpha} \omega \times \mathbf{N}\right)+\varrho v_{y} \cdot \nabla_{y}\left(\mathcal{Z}^{\alpha} \omega \times \mathbf{N}\right)+\varrho V_{z} \partial_{z}\left(\mathcal{Z}^{\alpha} \omega \times \mathbf{N}\right)-\mu \varepsilon \Delta^{\varphi}\left(\mathcal{Z}^{\alpha} \omega \times \mathbf{N}\right)=F,
$$

where the source term $F$ is given by

$$
F=\mathcal{Z}^{\alpha} F_{1} \times \mathbf{N}+F_{2} \times \mathbf{N}+F_{3},
$$

with $F_{3}$ given by

$$
F_{3}=\varrho \mathcal{Z}^{\alpha} \omega \times \partial_{t} \mathbf{N}+\varrho v_{y} \cdot \mathcal{Z}^{\alpha} \omega \times \nabla_{y} \mathbf{N}+\varrho V_{z} \mathcal{Z}^{\alpha} \omega \times \partial_{z} \mathbf{N}+\mu \varepsilon \mathcal{Z}^{\alpha} \omega \times \Delta^{\varphi} \mathbf{N}+2 \mu \varepsilon \nabla^{\varphi} \mathcal{Z}^{\alpha} \omega \times \nabla^{\varphi} \mathbf{N}
$$

Next, we follow the argument in [32], where $\mathcal{Z}^{m-1} \omega$ is bounded, to perform the estimate of $\mathcal{Z}^{m-1} \omega \times \mathbf{N}$ as follows. 
Proposition 4.18 For any $t \in\left[0, T^{\varepsilon}\right]$ and $|\alpha|=m-1$, there exists $\Lambda(M(t))$ such that

$$
\begin{aligned}
\left\|\mathcal{Z}^{\alpha} \omega \times \mathbf{N}\right\|_{L^{4}\left(0, t ; L^{2}(\mathcal{S})\right) \leq}^{2} & \Lambda_{0}\left\|\mathcal{Z}^{\alpha} \omega_{0}\right\|^{2}+\Lambda_{0} \varepsilon \int_{0}^{t}\left\|\nabla V^{m}\right\|^{2}+\left\|\nabla^{2} v\right\|_{\mathcal{H}^{m-2}}^{2} d \tau \\
& +\Lambda(M(t)) \int_{0}^{t} \Lambda\left(Y_{m}(\tau)\right) d \tau+\Lambda_{\infty}(t) t^{\frac{1}{2}} \int_{0}^{t}\|\nabla(p, v)(\tau)\|_{\mathcal{H}^{m-1}}^{2} d \tau
\end{aligned}
$$

where $M(t)$ is defined in 4.5.7].

The proof of this Proposition will be a consequence of the following lemmas. As in 32 , we split $\mathcal{Z}^{\alpha} \omega \times \mathbf{N}$ into two parts of the form

$$
\mathcal{Z}^{\alpha} \omega \times \mathbf{N}=\omega_{h}^{\alpha}+\omega_{n h}^{\alpha}
$$

where $\omega_{n h}^{\alpha}$ solves the non-homogeneous problem:

$$
\left\{\begin{array}{l}
\varrho \partial_{t} \omega_{n h}^{\alpha}+\varrho v_{y} \cdot \nabla_{y} \omega_{n h}^{\alpha}+\varrho V_{z} \partial_{z} \omega_{n h}^{\alpha}-\mu \varepsilon \Delta^{\varphi} \omega_{n h}^{\alpha}=F \\
\left.\omega_{n h}^{\alpha}\right|_{t=0}=\mathcal{Z}^{\alpha} \omega_{0} \times \mathbf{N}_{0} \\
\left.\omega_{n h}^{\alpha}\right|_{z=0}=0
\end{array}\right.
$$

while $\omega_{h}^{\alpha}$ solves the homogeneous one:

$$
\left\{\begin{array}{l}
\varrho \partial_{t} \omega_{h}^{\alpha}+\varrho v_{y} \cdot \nabla_{y} \omega_{h}^{\alpha}+\varrho V_{z} \partial_{z} \omega_{h}^{\alpha}-\mu \varepsilon \Delta^{\varphi} \omega_{h}^{\alpha}=0, \\
\left.\omega_{n}^{\alpha}\right|_{t=0}=0 \\
\left.\omega_{n}^{\alpha}\right|_{z=0}=\left(\mathcal{Z}^{\alpha} \omega \times \mathbf{N}\right)^{b} .
\end{array}\right.
$$

The solution $\omega_{n h}^{\alpha}$ to (4.5.20) can be estimated by standard energy estimates.

Lemma 4.19 For any $|\alpha| \leq m-1$, it holds that

$$
\begin{aligned}
\left\|\omega_{n h}^{\alpha}\right\|^{2}+\mu \varepsilon \int_{0}^{t}\left\|\nabla^{\varphi} \omega_{n h}^{\alpha}\right\|^{2} d \tau \leq & \Lambda_{0}\left\|\mathcal{Z}^{\alpha} \omega_{0}\right\|^{2}+\Lambda_{0} \varepsilon \int_{0}^{t}\left\|\nabla^{2} v\right\|_{\mathcal{H}^{m-2}}^{2} d \tau \\
& +\Lambda_{\infty}(t) \int_{0}^{t} \varepsilon\left|\mathcal{Z}^{m} h\right|_{\frac{1}{2}}^{2}+\|\nabla(p, v)\|_{\mathcal{H}^{m-1}}^{2}+\Lambda\left(Y_{m}(\tau)\right) d \tau .
\end{aligned}
$$

Proof. Noting the homogeneous Dirichlet boundary condition for $\omega_{n h}^{\alpha}$, one deduces from (4.5.20) by a standard energy estimate that

$$
\frac{1}{2} \int_{\mathcal{S}} \varrho\left|\omega_{n h}^{\alpha}\right|^{2} d \mathcal{V}_{\tau}+\mu \varepsilon \int_{0}^{t} \int_{\mathcal{S}}\left|\nabla^{\varphi} \omega_{n h}^{\alpha}\right| d \mathcal{V}_{\tau} d \tau \leq \Lambda_{0} \int_{\mathcal{S}} \varrho_{0}\left|\mathcal{Z}^{\alpha} \omega_{0}\right|^{2} d \mathcal{V}_{\tau}+\left|\int_{0}^{t} \int_{\mathcal{S}} F \cdot \omega_{n h}^{\alpha} d \mathcal{V}_{\tau} d \tau\right|
$$

where $F$ is given by (4.5.16). Then (2.1) implies that

$$
\begin{aligned}
& \left|\int_{0}^{t} \int_{\mathcal{S}} \mathcal{Z}^{\alpha} F_{1} \times \mathbf{N} \cdot \omega_{n h}^{\alpha} d \mathcal{V}_{\tau} d \tau\right|+\int_{0}^{t} \int_{\mathcal{S}}\left|F_{3} \cdot \omega_{n h}^{\alpha}\right|^{2} d \mathcal{V}_{\tau} d \tau \\
& \leq \frac{1}{16} \sup _{0 \leq \tau \leq t} \int\left|\omega_{n h}^{\alpha}(\tau)\right|^{2} d \mathcal{V}_{\tau}+\Lambda_{\infty}(t) \int_{0}^{t}\|(p, v)\|_{\mathcal{H}^{m}}^{2}+\|(\nabla p, \nabla v)\|_{\mathcal{H}^{m-1}}^{2}+\left|\mathcal{Z}^{m-1} h\right|_{\frac{1}{2}}^{2} d \tau
\end{aligned}
$$

Using similar arguments as $[32$, we can obtain that

$$
\begin{aligned}
& \int_{0}^{t} \int_{\mathcal{S}}\left|F_{2} \times \mathbf{N} \cdot \omega_{n h}^{\alpha}\right| d \mathcal{V}_{\tau} d \tau \\
& \leq \frac{\mu}{8} \varepsilon \int_{0}^{t} \int\left|\nabla^{\varphi} \omega_{n h}^{\alpha}\right|^{2} d \mathcal{V}_{\tau} d \tau+\Lambda_{0} \varepsilon \int_{0}^{t}\left\|\nabla^{2} v\right\|_{\mathcal{H}^{m-1}}^{2} d \tau+\frac{1}{16} \sup _{0 \leq \tau \leq t} \int\left|\omega_{n h}^{\alpha}(\tau)\right|^{2} d \mathcal{V}_{\tau} \\
& \quad+\Lambda_{\infty}(t) \int_{0}^{t} \varepsilon\left|\mathcal{Z}^{m} h\right|_{\frac{1}{2}}^{2}+\|\nabla(p, v)\|_{\mathcal{H}^{m-1}}^{2}+\Lambda\left(Y_{m}(\tau)\right) d \tau .
\end{aligned}
$$

Substituting (4.5.24) and (4.5.25) into (4.5.23) proves Proposition 4.19, Thus, the proof is completed.

To treat $\omega_{h}$, we will modify the approach of microlocal analysis used in 32 to obtain that 
Lemma 4.20 For any $|\alpha|=m-1$, it holds that

$$
\left(\int_{0}^{t}\left\|\omega_{h}^{\alpha}(\tau)\right\|^{4} d \tau\right)^{\frac{1}{2}} \leq \varepsilon \int_{0}^{t}\left\|\nabla V^{m}\right\|^{2}+\left\|\nabla^{2} v\right\|_{\mathcal{H}^{m-2}}^{2} d \tau+\Lambda(M(t)) \int_{0}^{t} \Lambda\left(Y_{m}(\tau)\right) d \tau
$$

where $M(t)$ is defined in 4.5.7).

In order to prove the above lemma, we follow the argument in 32 to use Lagrangian coordinates to eliminate the convection term so that it is convenient to perform the microlocal symmetrizer method. Let us define a parametrization by

$$
\partial_{t} X(t, y, z)=u(t, X(t, y, z))=v\left(t, \Phi(t, \cdot)^{-1} \circ X\right), \quad X(0, y, z)=\Phi(0, y, z),
$$

where $\Phi(t, \cdot)^{-1}$ stands for the inverse of the map $\Phi(t, \cdot)$ defined by (1.24). Define $J(t, y, z)=|\operatorname{det} \nabla X(t, y, z)|$ to be the Jacobian of the change of variable. The following estimates for $X$ are proved in Lemma 10.5 in 32]:

Lemma 4.21 It holds that for $t \in[0, T]$,

$$
\begin{aligned}
& \|\nabla X(t)\|_{L^{\infty}}+\left\|\partial_{t} \nabla X(t)\right\|_{L^{\infty}} \leq \Lambda_{0} e^{t \Lambda(M(t))}, \\
& \|\nabla X(t)\|_{1, \infty}+\left\|\partial_{t} \nabla X(t)\right\|_{1, \infty} \leq \Lambda(M(t)) e^{t \Lambda(M(t))}, \\
& \sqrt{\varepsilon}\left\|\nabla^{2} X\right\|_{1, \infty}+\sqrt{\varepsilon}\left\|\partial_{t} \nabla^{2} X\right\|_{L^{\infty}} \leq \Lambda(M(t))\left(1+t^{2}\right) e^{t \Lambda(M(t))} .
\end{aligned}
$$

Now set

$$
\Omega^{\alpha}=e^{-\gamma t} \omega_{h}^{\alpha}\left(t, \Phi^{-1} \circ X\right)
$$

where $\gamma>0$ is a large parameter to be chosen. Then $\Omega^{\alpha}$ solves in $\mathcal{S}$ the equation

$$
a_{0}\left(\partial_{t} \Omega^{\alpha}+\gamma \Omega^{\alpha}\right)-\mu \varepsilon \partial_{i}\left(a_{i j} \partial_{j} \Omega^{\alpha}\right)=0
$$

where

$$
a_{0}=\varrho\left(t, \Phi^{-1} \circ X\right)|J|^{\frac{1}{2}}, \quad\left(a_{i j}\right)=|J|^{\frac{1}{2}} P^{-1}, \quad P_{i j}=\partial_{i} X \cdot \partial_{j} X .
$$

Thanks to Lemma 4.21, the equation 4.5.32) is a parabolic equation. On the boundary, it holds that

$$
\left.\Omega^{\alpha}\right|_{z=0}=\left(\Omega^{\alpha}\right)^{b}:=e^{-\gamma t}\left(\mathcal{Z}^{\alpha} \omega \times \mathbf{N}\right)\left(t,\left(\Phi^{-1} \circ X\right)(t, y, 0) .\right.
$$

The following theorem holds:

Theorem 4.22 (Masmoudi-Rousset [32]) There exists $\gamma_{0}$ depending only on $M>0$ such that for $\gamma \geq \gamma_{0}$, the solution of (4.5.32) with the boundary condition (4.5.34) satisfies the estimate

$$
\left\|\Omega^{m-1}\right\|_{H^{\frac{1}{4}}\left(0, T ; L^{2}(\mathcal{S})\right)}^{2} \leq \Lambda(M(T)) \sqrt{\varepsilon} \int_{0}^{T}\left|\left(\Omega^{m-1}\right)^{b}\right|_{L^{2}\left(\mathbb{R}^{2}\right)}^{2},
$$

where the norm $H^{\frac{1}{4}}\left(0, T ; L^{2}\right)$ is defined by

$$
\|f\|_{H^{\frac{1}{4}\left(0, T ; L^{2}\right)}}=\inf \left\{\|P f\|_{H^{\frac{1}{4}\left(\mathbb{R}, L^{2}(\mathcal{S})\right)}}, P f=f \quad \text { on }[0, T] \times \mathcal{S}\right\},
$$

with the norm on the whole space by Fourier transform in time.

Proof of Theorem 4.22. The proof of this Theorem is almost the same as the proof of Theorem 10.6 in 32 where the symmetrized method and paradifferential calculus are used. This is because the coefficients of (4.5.32) given in (4.5.33) is the same type (determined by the estimate in Lemma 4.21. $\|(\varrho, \nabla \varrho)\|_{1, \infty} \leq M$ and initial data which ensure $\left.\Phi_{0}\right)$ of symbols as the one in [32] which is essential in the progress of taking use of paradifferential calculus. We refer to Theorem 10.6 in 32 for more details. 
Proof of Lemma 4.20 As a consequence of Theorem 4.22 and the Sobolev embedding inequality, one has that

$$
\left\|\Omega^{\alpha}\right\|_{L^{4}\left(0, T ; L^{2}(S)\right)}^{2} \leq C\left\|\Omega^{m-1}\right\|_{H^{\frac{1}{4}}\left(0, T ; L^{2}(S)\right)}^{2} \leq \Lambda(M(T)) \sqrt{\varepsilon} \int_{0}^{T}\left|\left(\Omega^{m-1}\right)^{b}\right|_{L^{2}\left(\mathbb{R}^{2}\right)}^{2}
$$

Consequently, it follows from changing of variable and (4.5.5) that

$$
\begin{aligned}
\left\|\omega_{h}^{\alpha}\right\|_{L^{4}\left(0, t ; L^{2}(S)\right)}^{2} & \leq \Lambda(M(t)) \sqrt{\varepsilon} \int_{0}^{t}\left|\left(\mathcal{Z}^{\alpha} \omega \times \mathbf{N}\right)^{b}\right|_{L^{2}\left(\mathbb{R}^{2}\right)}^{2} \\
& \leq \varepsilon \int_{0}^{t}\|\nabla v\|_{\mathcal{H}^{m}}^{2}+\left\|\nabla^{2} v\right\|_{\mathcal{H}^{m-2}}^{2} d \tau+\Lambda(M(t)) \int_{0}^{t} Y_{m}(\tau) d \tau .
\end{aligned}
$$

which yields Proposition 4.20 Therefore, the proof is completed.

\section{6 $\quad L^{\infty}$-estimates}

In order to close the estimates, one needs to bound the $L^{\infty}$ norms of $v, h$ and $p$ contained in $\Lambda_{\infty}(t)$. We start with the following estimates through standard Sobolev embedding theorem and anisotropic Sobolev embedding theorem.

Lemma 4.23 For any $t \in\left[0, T^{\varepsilon}\right]$, the following estimates hold:

$$
\begin{aligned}
& |h(t)|_{\mathcal{H}^{k, \infty}} \lesssim\left|\mathcal{Z}^{k} h(t)\right|_{\frac{3}{2}}, k \in \mathbb{N}, \\
& \|(p, v)(t)\|_{\mathcal{H}^{2, \infty}}^{2} \leq \Lambda\left(\frac{1}{c_{0}}, \mathcal{Q}_{5}(t)\right), \\
& \|\nabla p(t)\|_{\mathcal{H}^{1, \infty}}^{2} \leq\left\|\Delta^{\varphi} p(t)\right\|_{\mathcal{H}^{1}}^{2}+\Lambda\left(\frac{1}{c_{0}}, \mathcal{Q}_{5}(t)\right)\|\nabla p(t)\|_{\mathcal{H}^{4}}^{2}, \\
& \left\|d i v^{\varphi} v(t)\right\|_{\mathcal{H}^{1, \infty}}^{2} \leq\left\|\Delta^{\varphi} p(t)\right\|_{\mathcal{H}^{1}}^{2}+\Lambda\left(\frac{1}{c_{0}}, \mathcal{Q}_{5}(t)\right)\left(1+\|\nabla p(t)\|_{\mathcal{H}^{4}}^{2}\right), \\
& \left\|\nabla d i v^{\varphi} v(t)\right\|_{\infty}^{2} \leq\left\|\Delta^{\varphi} p\right\|_{\mathcal{H}^{1}}^{2}+\Lambda\left(\frac{1}{c_{0}}, \mathcal{Q}_{5}(t)+\|\nabla v(t)\|_{\infty}^{2}\right)\left(1+\|\nabla p\|_{\mathcal{H}^{4}}^{2}\right), \\
& \left\|\nabla d i v^{\varphi} v(t)\right\|_{\mathcal{H}^{1, \infty}}^{2} \leq \delta\left\|\Delta^{\varphi} p(t)\right\|_{\mathcal{H}^{2}}^{2}+C_{\delta} \Lambda\left(\frac{1}{c_{0}}, \mathcal{Q}_{6}(t)+\|\nabla v(t)\|_{\mathcal{W}^{1, \infty}}^{2}\right)\left(1+\|\nabla p\|_{\mathcal{H}^{5}}^{2}\right) .
\end{aligned}
$$

Proof. (4.6.1) follows from the two dimensional Sobolev embedding. The anisotropic Sobolev embedding (2.4) yields that

$$
\|(p, v)(t)\|_{\mathcal{H}^{2, \infty}}^{2} \lesssim\|(\nabla p, \nabla v)(t)\|_{\mathcal{H}^{3}}\|(p, v)(t)\|_{\mathcal{H}^{4}} \leq \Lambda\left(\frac{1}{c_{0}}, \mathcal{Q}_{5}(t)\right) .
$$

To prove (4.6.3), one notes that

$$
\Delta^{\varphi}=\frac{1+\left|\nabla_{y} \varphi\right|^{2}}{\left|\partial_{z} \varphi\right|^{2}} \partial_{z z}+\sum_{i=1,2}\left(\partial_{i i}-\frac{2 \partial_{i} \varphi}{\partial_{z} \varphi} \partial_{i} \partial_{z}-2 \partial_{i}\left(\frac{\partial_{i} \varphi}{\partial_{z} \varphi}\right) \partial_{z}+\frac{\partial_{i} \varphi}{\partial_{z} \varphi} \partial_{z}\left(\frac{\partial_{i} \varphi}{\partial_{z} \varphi}\right) \partial_{z}+\frac{1}{\partial_{z} \varphi} \partial_{z}\left(\frac{1}{\partial_{z} \varphi}\right) \partial_{z}\right),
$$

which follows from the definition of $\partial_{i}^{\varphi}$ immediately. Thus, (2.4) implies that

$$
\begin{aligned}
\|\nabla p(t)\|_{\infty}^{2} & \lesssim\|\nabla p(t)\|_{3}\left\|\partial_{z} \nabla p(t)+\right\| \nabla p(t)\left\|_{3}^{2}\right\| \lesssim\|\nabla p(t)\|_{3}\left(\left\|\partial_{z} p(t)\right\|_{3}+\left\|\partial_{z z} p(t)\right\|\right) \\
& \lesssim \Lambda\left(\frac{1}{c_{0}},|h(t)|_{2, \infty}\right)\|\nabla p(t)\|_{3}\left(\|\nabla p(t)\|_{3}+\|p(t)\|_{2}+\left\|\Delta^{\varphi} p(t)\right\|\right) \\
& \leq\left\|\Delta^{\varphi} p(t)\right\|^{2}+\Lambda\left(\frac{1}{c_{0}}, \mathcal{Q}_{5}(t)\right) .
\end{aligned}
$$

Similarly,

$$
\begin{aligned}
\|\mathcal{Z} \nabla p(t)\|_{\infty}^{2} & \lesssim\|\mathcal{Z} \nabla p\|_{3}\left\|\partial_{z} \mathcal{Z} \nabla p\right\|+\|\nabla p\|_{4}^{2} \\
& \lesssim \Lambda\left(\frac{1}{c_{0}},|h(t)|_{3, \infty}\right)\|\nabla p(t)\|_{\mathcal{H}^{4}}\left(\|\nabla p(t)\|_{\mathcal{H}^{4}}+\|p(t)\|_{\mathcal{H}^{3}}+\left\|\Delta^{\varphi} p(t)\right\|_{\mathcal{H}^{1}}\right) \\
& \leq\left\|\Delta^{\varphi} p(t)\right\|_{\mathcal{H}^{1}}^{2}+\Lambda\left(\frac{1}{c_{0}}, \mathcal{Q}_{5}(t)\right)\|\nabla p\|_{\mathcal{H}^{4}}^{2} .
\end{aligned}
$$


Therefore, (4.6.3) holds true. Next, it follows from (4.3.5), (4.6.2) and (4.6.3) that

$$
\begin{aligned}
\left\|\operatorname{div}^{\varphi} v(t)\right\|_{\mathcal{H}^{1, \infty}}^{2} & \lesssim\left\|\frac{1}{p}(t)\right\|_{\mathcal{H}^{1, \infty}}^{2}\left(\|p(t)\|_{\mathcal{H}^{2, \infty}}^{2}+\left\|v_{y}(t)\right\|_{\mathcal{H}^{1, \infty}}^{2}\|p(t)\|_{\mathcal{H}^{2, \infty}}^{2}+\left\|V_{z}(t)\right\|_{\mathcal{H}^{1, \infty}}^{2}\left\|\partial_{z} p\right\|_{\mathcal{H}^{1, \infty}}^{2}\right) \\
& \lesssim \Lambda\left(\frac{1}{c_{0}}, \mathcal{Q}_{5}(t)\right)\left\{1+\|\nabla p(t)\|_{\mathcal{H}^{4}}\left[\|\nabla p(t)\|_{\mathcal{H}^{4}}+\|p(t)\|_{\mathcal{H}^{3}}+\left\|\Delta^{\varphi} p(t)\right\|_{\mathcal{H}^{1}}\right]\right\} \\
& \leq\left\|\Delta^{\varphi} p(t)\right\|_{\mathcal{H}^{1}}^{2}+\Lambda\left(\frac{1}{c_{0}}, \mathcal{Q}_{5}(t)\right)\left(1+\|\nabla p\|_{\mathcal{H}^{4}}^{2}\right) .
\end{aligned}
$$

Furthermore, it follows from the relation

$$
\nabla \operatorname{div}^{\varphi} v=-\frac{1}{\gamma p}\left(\partial_{t} \nabla p+v_{y} \cdot \nabla_{y} \nabla p+V_{z} \partial_{z} \nabla p\right)-\nabla\left(\frac{1}{\gamma p}\right) \partial_{t} p-\nabla\left(\frac{v_{y}}{\gamma p}\right) \cdot \nabla_{y} p-\nabla\left(\frac{V_{z}}{\gamma p}\right) \partial_{z} p,
$$

that

$$
\begin{aligned}
\| & \nabla \operatorname{div}^{\varphi} v(t) \|_{\infty}^{2} \\
\leq & \Lambda_{0}\left\{\|\nabla p(t)\|_{\mathcal{H}^{1, \infty}}^{2}+\left\|v_{y}(t)\right\|_{\infty}^{2}\|\nabla p(t)\|_{\mathcal{H}^{1, \infty}}^{2}+\left\|\left(V_{z} \partial_{z} \nabla p\right)(t)\right\|_{\infty}^{2}+\|\nabla p(t)\|_{\infty}^{2}\|p(t)\|_{\mathcal{H}^{1, \infty}}^{2}\right. \\
& \left.+\left(\left\|\nabla v_{y}(t)\right\|_{\infty}^{2}+\left\|\left(v_{y} \nabla p\right)(t)\right\|_{\infty}^{2}\right)\|p(t)\|_{\mathcal{H}^{1, \infty}}^{2}+\left(\left\|\nabla V_{z}(t)\right\|_{\infty}^{2}+\left\|\left(V_{z} \nabla p\right)(t)\right\|_{\infty}^{2}\right)\left\|\partial_{z} p(t)\right\|_{\infty}^{2}\right\} \\
\leq & \Lambda\left(\frac{1}{c_{0}}, \mathcal{Q}_{5}(t)\right)\left(1+\|\nabla v\|_{\infty}^{2}\right)\left(1+\|\nabla p\|_{\mathcal{H}^{1, \infty}}^{2}\right) \\
\leq & \left\|\Delta^{\varphi} p\right\|_{\mathcal{H}^{1}}^{2}+\Lambda\left(\frac{1}{c_{0}}, \mathcal{Q}_{5}(t)+\|\nabla v(t)\|_{\infty}^{2}\right)\left(1+\|\nabla p\|_{\mathcal{H}^{4}}^{2}\right),
\end{aligned}
$$

since $V_{z}$ vanishes on the boundary. Similarly,

$$
\left\|\nabla \operatorname{div}^{\varphi} v(t)\right\|_{\mathcal{H}^{1, \infty}}^{2} \leq \delta\left\|\Delta^{\varphi} p(t)\right\|_{\mathcal{H}^{2}}^{2}+C_{\delta} \Lambda\left(\frac{1}{c_{0}}, \mathcal{Q}_{6}(t)+\|\nabla v(t)\|_{\mathcal{H}^{1, \infty}}^{2}\right)\left(1+\|\nabla p\|_{\mathcal{H}^{5}}^{2}\right) .
$$

Therefore, the proof of this lemma is completed.

We now estimate $\left\|\Delta^{\varphi} p\right\|_{\mathcal{H}^{1}}^{2}$. Applying $\operatorname{div}^{\varphi}$ to the momentum equations yields that

$$
-(2 \mu+\lambda) \varepsilon \Delta^{\varphi} \operatorname{div}^{\varphi} v+\Delta^{\varphi} p=\operatorname{div}^{\varphi}\left(\varrho \partial_{t}^{\varphi} v+\varrho v \cdot \nabla^{\varphi} v\right)
$$

This, together with (4.3.5) and (3.15) shows that

$$
\begin{gathered}
\varepsilon \partial_{t} \Delta^{\varphi} p+\frac{\gamma p}{2 \mu+\lambda} \Delta^{\varphi} p=\frac{\gamma p}{2 \mu+\lambda} \operatorname{div}^{\varphi}\left(\varrho \partial_{t}^{\varphi} v+\varrho v \cdot \nabla^{\varphi} v\right)-\varepsilon\left(v_{y} \nabla_{y}+V_{z} \partial_{z}\right) \Delta^{\varphi} p \\
-\varepsilon \gamma p\left(\partial_{t}^{\varphi} p \Delta^{\varphi}\left(\frac{1}{\gamma p}\right)+\nabla^{\varphi} p \Delta^{\varphi}\left(\frac{v}{\gamma p}\right)+2 \nabla^{\varphi} \partial_{t}^{\varphi} p \nabla^{\varphi}\left(\frac{1}{\gamma p}\right)+2 \nabla^{\varphi}\left(\frac{v}{\gamma p}\right) \nabla^{\varphi} \nabla^{\varphi} p\right) .
\end{gathered}
$$

Lemma 4.24 For $m \geq 6$, it holds that

$$
\begin{aligned}
& \sup _{0 \leq \tau \leq t}\left(\left\|\Delta^{\varphi} p(\tau)\right\|_{\mathcal{H}^{1}}^{2}+\varepsilon\left\|\Delta^{\varphi} p(\tau)\right\|_{\mathcal{H}^{2}}^{2}\right)+\int_{0}^{t}\left\|\Delta^{\varphi} p\right\|_{\mathcal{H}^{2}}^{2} d \tau \\
& \leq C \Lambda_{0}\left(\left\|\left(\Delta^{\varphi} p\right)(0)\right\|_{\mathcal{H}^{1}}^{2}+\varepsilon\left\|\left(\Delta^{\varphi} p\right)(0)\right\|_{\mathcal{H}^{2}}^{2}\right)+\Lambda_{\infty}(t) \int_{0}^{t} \Lambda\left(Y_{m}(\tau)\right) d \tau
\end{aligned}
$$


Proof. Applying $\mathcal{Z}^{\alpha}(|\alpha|=2)$ to (4.6.14), multiplying the resulting equation by $\mathcal{Z}^{\alpha} \Delta^{\varphi} p$ and then integrating over $\mathcal{S} \times[0, t]$, one can get that

$$
\begin{aligned}
\frac{1}{2} \varepsilon\left\|\mathcal{Z}^{\alpha} \Delta^{\varphi} p\right\|^{2}+\frac{1}{2 \mu+\lambda} \int_{0}^{t} \int_{\mathcal{S}} \mathcal{Z}^{\alpha}\left(\gamma p \Delta^{\varphi} p\right) \mathcal{Z}^{\alpha} \Delta^{\varphi} p d \mathcal{V}_{\tau} d \tau \\
=\frac{\varepsilon}{2}\left\|\left(\mathcal{Z}^{\alpha} \Delta^{\varphi} p\right)(0)\right\|^{2}-\varepsilon \int_{0}^{t} \int_{\mathcal{S}} \mathcal{Z}^{\alpha}\left(v_{y} \cdot \nabla_{y} \Delta^{\varphi} p+V_{z} \partial_{z} \Delta^{\varphi} p\right) \mathcal{Z}^{\alpha} \Delta^{\varphi} p d \mathcal{V}_{\tau} d \tau \\
\quad-\varepsilon \int_{0}^{t} \int_{\mathcal{S}} \mathcal{Z}^{\alpha}\left(\gamma p \partial_{t}^{\varphi} p \Delta^{\varphi}\left(\frac{1}{\gamma p}\right)\right) \mathcal{Z}^{\alpha} \Delta^{\varphi} p d \mathcal{V}_{\tau} d \tau-\varepsilon \int_{0}^{t} \int_{\mathcal{S}} \mathcal{Z}^{\alpha}\left(\gamma p \nabla^{\varphi} p \Delta^{\varphi}\left(\frac{v}{\gamma p}\right)\right) \mathcal{Z}^{\alpha} \Delta^{\varphi} p d \mathcal{V}_{\tau} d \tau \\
\quad-2 \varepsilon \int_{0}^{t} \int_{\mathcal{S}} \mathcal{Z}^{\alpha}\left(\gamma p\left(\nabla^{\varphi} \partial_{t}^{\varphi} p \nabla^{\varphi}\left(\frac{1}{\gamma p}\right)+\nabla^{\varphi}\left(\frac{v}{\gamma p}\right) \nabla^{\varphi} \nabla^{\varphi} p\right)\right) \mathcal{Z}^{\alpha} \Delta^{\varphi} p d \mathcal{V}_{\tau} d \tau \\
-\frac{1}{2 \mu+\lambda} \int_{0}^{t} \int_{\mathcal{S}} \mathcal{Z}^{\alpha}\left\{\gamma p \operatorname{div}^{\varphi}\left(\varrho\left(\partial_{t}^{\varphi}+v \cdot \nabla^{\varphi}\right) v\right)\right\} \mathcal{Z}^{\alpha} \Delta^{\varphi} p d \mathcal{V}_{\tau} d \tau=: \frac{\varepsilon}{2}\left\|\left(\mathcal{Z}^{\alpha} \Delta^{\varphi} p\right)(0)\right\|^{2}+\sum_{i=1}^{5} J_{i}
\end{aligned}
$$

The second term on the LHS above can be estimated directly as

$$
\begin{aligned}
& \frac{1}{2 \mu+\lambda} \int_{0}^{t} \int_{\mathcal{S}} \mathcal{Z}^{\alpha}\left(\gamma p \Delta^{\varphi} p\right) \mathcal{Z}^{\alpha} \Delta^{\varphi} p d \mathcal{V}_{\tau} d \tau \geq \frac{1}{2 \mu+\lambda} \int_{0}^{t} \int_{\mathcal{S}} \gamma p\left|\mathcal{Z}^{\alpha} \Delta^{\varphi} p\right|^{2} d \mathcal{V}_{\tau} d \tau \\
& \quad+\frac{2}{2 \mu+\lambda} \int_{0}^{t} \int_{\mathcal{S}} \gamma \mathcal{Z} p \mathcal{Z}\left(\Delta^{\varphi} p\right) \mathcal{Z}^{\alpha}\left(\Delta^{\varphi} p\right) d \mathcal{V}_{\tau} d \tau+\frac{1}{2 \mu+\lambda} \int_{0}^{t} \int_{\mathcal{S}} \gamma \mathcal{Z}^{\alpha} p \Delta^{\varphi} p \mathcal{Z}^{\alpha}\left(\Delta^{\varphi} p\right) d \mathcal{V}_{\tau} d \tau \\
& \geq \frac{3}{4(2 \mu+\lambda)} \int_{0}^{t} \int_{\mathcal{S}} \gamma p\left|\mathcal{Z}^{\alpha} \Delta^{\varphi} p\right|^{2} d \mathcal{V}_{\tau} d \tau-\Lambda_{0} \int_{0}^{t}\|\mathcal{Z} p\|_{\infty}^{2}\left\|\Delta^{\varphi} p\right\|_{\mathcal{H}^{1}}^{2}+\left\|\mathcal{Z}^{\alpha} p\right\|_{\infty}^{2}\left\|\Delta^{\varphi} p\right\|^{2} d \tau \\
& \geq \frac{3}{4(2 \mu+\lambda)} \int_{0}^{t} \int_{\mathcal{S}} \gamma p\left|\mathcal{Z}^{\alpha} \Delta^{\varphi} p\right|^{2} d \mathcal{V}_{\tau} d \tau-\int_{0}^{t} \Lambda\left(\frac{1}{c_{0}}, \mathcal{Q}_{m}(t)+\left\|\Delta^{\varphi} p\right\|_{\mathcal{H}^{1}}^{2}\right) d \tau
\end{aligned}
$$

where $m \geq 5$. Next, the terms on the RHS of (4.6.16) can be estimated separately. First, it follows from integration by parts, (2.2) and (3.25) that

$$
\begin{aligned}
\left|J_{1}\right| \leq & \varepsilon\left|\int_{0}^{t} \int_{\mathcal{S}}\left(v_{y} \nabla_{y} \mathcal{Z}^{\alpha} \Delta^{\varphi} p+V_{z} \partial_{z} \mathcal{Z}^{\alpha} \Delta^{\varphi} p\right) \mathcal{Z}^{\alpha} \Delta^{\varphi} p d \mathcal{V}_{\tau} d \tau\right| \\
& +\varepsilon\left|\int_{0}^{t} \int_{\mathcal{S}}\left(\left[\mathcal{Z}^{\alpha}, v_{y} \nabla_{y}\right] \Delta^{\varphi} p+\left[\mathcal{Z}^{\alpha}, V_{z}\right] \partial_{z} \Delta^{\varphi} p+V_{z}\left[\mathcal{Z}^{\alpha}, \partial_{z}\right] \Delta^{\varphi} p\right) \mathcal{Z}^{\alpha} \Delta^{\varphi} p d \mathcal{V}_{\tau} d \tau\right| \\
\leq & \Lambda_{\infty}(t) \int_{0}^{t} \varepsilon\left\|\Delta^{\varphi} p\right\|_{\mathcal{H}^{2}}^{2} d \tau+\varepsilon \int_{0}^{t}\left\|\frac{1-z}{z} \mathcal{Z}^{\alpha} V_{z}\right\|_{\infty}^{2}\left\|\Delta^{\varphi} p\right\|_{\mathcal{H}^{1}}^{2}+\left\|\frac{1-z}{z} \mathcal{Z} V_{z}\right\|_{\infty}^{2}\left\|\Delta^{\varphi} p\right\|_{\mathcal{H}^{2}}^{2} d \tau \\
\leq & \varepsilon^{2} \int_{0}^{t}\left\|\nabla^{2} v\right\|_{\mathcal{H}^{3}}^{2} d \tau+\Lambda_{\infty}(t) \int_{0}^{t} \varepsilon\left\|\Delta^{\varphi} p\right\|_{\mathcal{H}^{2}}^{2} d \tau+\int_{0}^{t} \Lambda\left(\frac{1}{c_{0}}, \mathcal{Q}_{m}(t)+\left\|\Delta^{\varphi} p\right\|_{\mathcal{H}^{1}}^{2}\right) d \tau \\
\leq & \Lambda_{\infty}(t) \int_{0}^{t} \Lambda\left(Y_{m}(\tau)\right) d \tau, \text { for } m \geq 6
\end{aligned}
$$

where (4.3.31) has been used in the last inequality.

Note that

$$
\Delta^{\varphi}\left(\frac{1}{p}\right)=\frac{2}{p^{3}}\left|\nabla^{\varphi} p\right|^{2}-\frac{\Delta^{\varphi} p}{p^{2}}, \quad \Delta^{\varphi}\left(\frac{v}{p}\right)=\frac{\Delta^{\varphi} v}{p}-\frac{2 \partial_{i}^{\varphi} v \partial_{i}^{\varphi} p}{p^{2}}+\frac{2 v}{p^{3}}\left|\nabla^{\varphi} p\right|^{2}-\frac{v}{p^{2}} \Delta^{\varphi} p,
$$

Thus, for $m \geq 6$, it holds that

$$
\begin{aligned}
& \left|J_{2}\right| \leq \frac{1}{16(2 \mu+\lambda)} \int_{0}^{t} \int_{\mathcal{S}} \gamma p\left|\mathcal{Z}^{\alpha} \Delta^{\varphi} p\right|^{2} d \mathcal{V}_{\tau} d \tau+C \varepsilon^{2} \int_{0}^{t}\left\|\mathcal{Z}^{\alpha}\left(\gamma p \partial_{t}^{\varphi} p \Delta^{\varphi}\left(\frac{1}{\gamma p}\right)\right)\right\|^{2} d \tau \\
& \leq \frac{1}{16(2 \mu+\lambda)} \int_{0}^{t} \int_{\mathcal{S}} \gamma p\left|\mathcal{Z}^{\alpha} \Delta^{\varphi} p\right|^{2} d \mathcal{V}_{\tau} d \tau+\Lambda_{\infty}(t) \int_{0}^{t} \Lambda\left(\frac{1}{c_{0}}, \mathcal{Q}_{m}(t)+\left\|\Delta^{\varphi} p\right\|_{\mathcal{H}^{1}}^{2}+\varepsilon\left\|\Delta^{\varphi} p\right\|_{\mathcal{H}^{2}}^{2}\right) d \tau \\
& \leq \frac{1}{16(2 \mu+\lambda)} \int_{0}^{t} \int_{\mathcal{S}} \gamma p\left|\mathcal{Z}^{\alpha} \Delta^{\varphi} p\right|^{2} d \mathcal{V}_{\tau} d \tau+\Lambda_{\infty}(t) \int_{0}^{t} \Lambda\left(Y_{m}(\tau)\right) d \tau
\end{aligned}
$$


and

$$
\begin{aligned}
\left|J_{3}\right| \leq & \varepsilon\left\{\int_{0}^{t}\left\|\mathcal{Z}^{\alpha} \Delta^{\varphi} p\right\|^{2} d \tau\right\}^{\frac{1}{2}} \\
& \times\left\{\int_{0}^{t}\left\|\mathcal{Z}^{\alpha} \nabla^{\varphi} p \cdot \Delta^{\varphi}\left(\frac{v}{p}\right)\right\|^{2}+\left\|\mathcal{Z} \nabla^{\varphi} p \mathcal{Z} \Delta^{\varphi}\left(\frac{v}{p}\right)\right\|^{2}+\left\|\nabla^{\varphi} p \mathcal{Z}^{\alpha} \Delta^{\varphi}\left(\frac{v}{p}\right)\right\|^{2} d \tau\right\}^{\frac{1}{2}} \\
\leq & \frac{1}{16(2 \mu+\lambda)} \int_{0}^{t} \int_{\mathcal{S}} \gamma p\left|\mathcal{Z}^{\alpha} \Delta^{\varphi} p\right|^{2} d \mathcal{V}_{\tau} d \tau+C \varepsilon^{2} \Lambda\left(\frac{1}{c_{0}}, \mathcal{Q}(t)+\mathcal{Q}_{m}\right) \int_{0}^{t}\left(\left\|\nabla^{\varphi} p\right\|_{\mathcal{H}^{2}}^{2}+\left\|\nabla^{\varphi} p \nabla^{\varphi} v\right\|_{\mathcal{H}^{2}}^{2}\right. \\
& \left.+\left\|\mathcal{Z}^{\alpha} \nabla^{\varphi} p\right\|_{2}^{2}\left\|\Delta^{\varphi} v\right\|_{\infty}^{2}+\left\|\mathcal{Z}^{\alpha} \nabla^{\varphi} p\right\|_{\infty}^{2}\left\|\Delta^{\varphi} p\right\|_{2}^{2}+\left\|\Delta^{\varphi}(v, p)\right\|_{\mathcal{H}^{2}}^{2}+\left\|\nabla^{\varphi}(p, v)\right\|_{\mathcal{H}^{1}}^{2}\right) d \tau \\
\leq & \frac{1}{16(2 \mu+\lambda)} \int_{0}^{t} \int_{\mathcal{S}} \gamma p\left|\mathcal{Z}^{\alpha} \Delta^{\varphi} p\right|^{2} d \mathcal{V}_{\tau} d \tau+C \Lambda\left(\frac{1}{c_{0}}, \mathcal{Q}(t)+\mathcal{Q}_{m}\right) \int_{0}^{t} \varepsilon^{2}\left\|\nabla^{2} v\right\|_{\mathcal{H}^{2}}^{2}+\Lambda\left(Y_{m}(\tau)\right) d \tau \\
\leq & \frac{1}{16(2 \mu+\lambda)} \int_{0}^{t} \int_{\mathcal{S}} \gamma p\left|\mathcal{Z}^{\alpha} \Delta^{\varphi} p\right|^{2} d \mathcal{V}_{\tau} d \tau+\Lambda_{\infty}(t) \int_{0}^{t} \Lambda\left(Y_{m}(\tau)\right) d \tau
\end{aligned}
$$

Similarly, for $m \geq 6$, it holds that

$$
\left|J_{4}\right| \leq \frac{1}{16(2 \mu+\lambda)} \int_{0}^{t} \int_{\mathcal{S}} \gamma p\left|\mathcal{Z}^{\alpha} \Delta^{\varphi} p\right|^{2} d \mathcal{V}_{\tau} d \tau+\Lambda_{\infty}(t) \int_{0}^{t} \Lambda\left(Y_{m}(\tau)\right) d \tau
$$

Next the expression

$$
\operatorname{div}^{\varphi}\left(\varrho\left(\partial_{t}^{\varphi}+v \cdot \nabla^{\varphi}\right) v\right)=\varrho \partial_{t} \operatorname{div}^{\varphi} v+\varrho v_{y} \nabla_{y} \operatorname{div}^{\varphi} v+\frac{1-z}{z} \varrho V_{z} Z_{3} \operatorname{div}^{\varphi} v+\nabla^{\varphi} \varrho \partial_{t}^{\varphi} v+\nabla^{\varphi}(\varrho v)^{t} \nabla^{\varphi} v,
$$

implies immediately, that for $m \geq 6$,

$$
\int_{0}^{t}\left\|p \operatorname{div}^{\varphi}\left(\varrho\left(\partial_{t}^{\varphi}+v \cdot \nabla^{\varphi}\right) v\right)\right\|_{\mathcal{H}^{2}}^{2} d \tau \leq \Lambda_{\infty}(t) \int_{0}^{t} \Lambda\left(Y_{m}(\tau)\right) d \tau
$$

Thus,

$$
\begin{aligned}
\left|J_{5}\right| & \leq \frac{1}{16(2 \mu+\lambda)} \int_{0}^{t} \int_{\mathcal{S}} \gamma p\left|\mathcal{Z}^{\alpha} \Delta^{\varphi} p\right|^{2} d \mathcal{V}_{\tau} d \tau+C \int_{0}^{t}\left\|p \operatorname{div}^{\varphi}\left(\varrho\left(\partial_{t}^{\varphi}+v \cdot \nabla^{\varphi}\right) v\right)\right\|_{\mathcal{H}^{2}}^{2} d \tau \\
& \leq \frac{1}{16(2 \mu+\lambda)} \int_{0}^{t} \int_{\mathcal{S}} \gamma p\left|\mathcal{Z}^{\alpha} \Delta^{\varphi} p\right|^{2} d \mathcal{V}_{\tau} d \tau+\Lambda_{\infty}(t) \int_{0}^{t} \Lambda\left(Y_{m}(\tau)\right) d \tau
\end{aligned}
$$

Substituting (4.6.17)-(4.6.23) into (4.6.16) shows that

$$
\varepsilon\left\|\Delta^{\varphi} p(\tau)\right\|_{\mathcal{H}^{2}}^{2}+\int_{0}^{t}\left\|\Delta^{\varphi} p\right\|_{\mathcal{H}^{2}}^{2} d \tau \leq \Lambda_{0} \varepsilon\left\|\Delta^{\varphi} p(0)\right\|_{\mathcal{H}^{2}}^{2}+\Lambda_{0} \int_{0}^{t} \Lambda\left(Y_{m}(\tau)\right) d \tau .
$$

On the other hand, it is easy to obtain that

$$
\begin{aligned}
\left\|\Delta^{\varphi} p\right\|_{\mathcal{H}^{1}}^{2} & \leq \Lambda_{0}\left\|\left(\Delta^{\varphi} p\right)(0)\right\|_{\mathcal{H}^{1}}^{2}+\Lambda_{0} \int_{0}^{t}\left\|\partial_{t}\left(\Delta^{\varphi} p\right)\right\|_{\mathcal{H}^{1}}^{2} d \tau \\
& \leq \Lambda_{0}\left\|\left(\Delta^{\varphi} p\right)(0)\right\|_{\mathcal{H}^{1}}^{2}+\Lambda_{0} \int_{0}^{t}\left\|\Delta^{\varphi} p\right\|_{\mathcal{H}^{2}}^{2} d \tau+C_{\delta} \Lambda_{\infty}(t) \int_{0}^{t} \Lambda\left(Y_{m}(\tau)\right) d \tau
\end{aligned}
$$

Then, combining (4.6.25) and (4.6.24) proves (4.6.15). Therefore, the proof of this lemma is completed.

We now turn to the most difficult part of $L^{\infty}$-estimates: the control of $\|\nabla v(t)\|_{\mathcal{H}^{1, \infty}}^{2}$ and $\varepsilon\left\|\partial_{z z} v(t)\right\|_{\infty}^{2}$. It follows from (4.4.1), (4.4.3), (4.4.6), (4.6.2), 4.6.4) and (4.6.15) that, for $m \geq 6$

$$
\begin{aligned}
\|\nabla v(t)\|_{\mathcal{H}^{1, \infty}}^{2} & \leq \Lambda\left(\frac{1}{c_{0}},|h(t)|_{\mathcal{H}^{3, \infty}}^{2}\right)\left(\left\|\operatorname{div}^{\varphi} v(t)\right\|_{\mathcal{H}^{1, \infty}}^{2}+\left\|S_{\mathbf{n}}(t)\right\|_{\mathcal{H}^{1, \infty}}^{2}+\|v(t)\|_{\mathcal{H}^{2, \infty}}^{2}\right) \\
& \leq\left\|\Delta^{\varphi} p\right\|_{\mathcal{H}^{1}}^{2}+\Lambda\left(\frac{1}{c_{0}}, \mathcal{Q}_{5}(t)\right)\left(1+\|\nabla p\|_{\mathcal{H}^{4}}^{2}\right)+\Lambda_{0}\left\|S_{\mathbf{n}}(t)\right\|_{\mathcal{H}^{1, \infty}}^{2} \\
& \leq \Lambda\left(Y_{6}(0)\right)+\Lambda_{0} t \sup _{0 \leq \tau \leq t} \Lambda\left(Y_{m}(\tau)\right)\left(1+\int_{0}^{t}\|\nabla p(\tau)\|_{\mathcal{H}^{5}}^{2} d \tau\right)+\Lambda_{0}\left\|S_{\mathbf{n}}(t)\right\|_{\mathcal{H}^{1, \infty}}^{2},
\end{aligned}
$$


where the following elementary estimate have been used

$$
\begin{aligned}
& \mathcal{Q}_{5}(t) \leq \mathcal{Q}_{5}(0)+C \int_{0}^{t} Y_{6}(\tau) d \tau, \\
& \|\nabla p(t)\|_{\mathcal{H}^{4}}^{2} \leq\|\nabla p(0)\|_{\mathcal{H}^{4}}^{2}+\int_{0}^{t}\|\nabla p(\tau)\|_{\mathcal{H}^{5}}^{2} d \tau .
\end{aligned}
$$

Similarly, one can get that

$$
\begin{aligned}
\varepsilon\left\|\partial_{z z} v\right\|_{\infty}^{2} & \leq \Lambda\left(\frac{1}{c_{0}},|h(t)|_{\mathcal{H}^{3, \infty}}^{2}\right) \varepsilon\left(\left\|\nabla \operatorname{div}^{\varphi} v(t)\right\|_{\infty}^{2}+\left\|\nabla S_{\mathbf{n}}(t)\right\|_{\infty}^{2}+\|\nabla v(t)\|_{\mathcal{H}^{1, \infty}}^{2}\right) \\
& \leq \Lambda_{0}\left\|\Delta^{\varphi} p\right\|_{\mathcal{H}^{1}}^{2}+\Lambda\left(\frac{1}{c_{0}}, \mathcal{Q}_{5}(t)+\|\nabla v(t)\|_{\infty}^{2}\right) \varepsilon\|\nabla p\|_{\mathcal{H}^{4}}^{2}+\Lambda_{0} \varepsilon\left(\left\|S_{\mathbf{n}}(t)\right\|_{\mathcal{H}^{1, \infty}}^{2}+\left\|\nabla S_{\mathbf{n}}(t)\right\|_{\infty}^{2}\right) \\
& \leq \Lambda\left(Y_{6}(0)\right)+\Lambda_{0} t \sup _{0 \leq \tau \leq t} \Lambda\left(Y_{m}(\tau)\right)+\Lambda_{0} \varepsilon\left(\left\|S_{\mathbf{n}}(t)\right\|_{\mathcal{H}^{1, \infty}}^{2}+\left\|\nabla S_{\mathbf{n}}(t)\right\|_{\infty}^{2}\right),
\end{aligned}
$$

Therefore, it suffices to bound $\left\|S_{\mathbf{n}}(t)\right\|_{\mathcal{H}^{1, \infty}}^{2}$ and $\varepsilon\left\|\nabla S_{\mathbf{n}}(t)\right\|_{\infty}^{2}$.

However, it seems difficult to bound $\left\|S_{\mathbf{n}}(t)\right\|_{\mathcal{H}^{1, \infty}}^{2}$ and $\varepsilon\left\|\nabla S_{\mathbf{n}}(t)\right\|_{\infty}^{2}$ by using the approach in 32. directly. This is because $\left(\nabla_{y}, 0\right)^{t}\left(\nabla^{\varphi} \operatorname{div}^{\varphi} v \cdot \mathbf{N}\right)$ must appear in (4.4.12) for compressible flows, but we can not expect to bound uniformly $\left\|\nabla \operatorname{div}^{\varphi} v\right\|_{\mathcal{H}^{1, \infty}}^{2}$ and $\varepsilon\left\|\nabla \operatorname{div}^{\varphi} v\right\|_{\mathcal{H}^{2, \infty}}^{2}$. Thus, we propose to estimate $\omega_{\mathbf{n}}$, which eliminates $\nabla^{\varphi} \operatorname{div}^{\varphi} v$ in its equation, instead of $S_{\mathbf{n}}$. Indeed, it follows from (4.5.2) that, for $m \geq 6$,

$$
\begin{aligned}
\left\|S_{\mathbf{n}}(t)\right\|_{\mathcal{H}^{1, \infty}}^{2} & \leq \Lambda_{0}\left\|\omega_{\mathbf{n}}(t)\right\|_{\mathcal{H}^{1, \infty}}^{2}+\Lambda\left(\frac{1}{c_{0}},|h(t)|_{\mathcal{H}^{3, \infty}}^{2}\right)\|v(t)\|_{\mathcal{H}^{2, \infty}}^{2} \\
& \leq \Lambda\left(\frac{1}{c_{0}} Y_{m}(0)\right)+\Lambda_{0}\left\|\omega_{\mathbf{n}}(t)\right\|_{\mathcal{H}^{1, \infty}}^{2}+C \int_{0}^{t} \Lambda\left(Y_{m}(\tau)\right) d \tau,
\end{aligned}
$$

and

$$
\begin{aligned}
\varepsilon\left\|\nabla S_{\mathbf{n}}(t)\right\|_{\infty}^{2} & \leq \Lambda_{0} \varepsilon\left\|\nabla \omega_{\mathbf{n}}\right\|_{\infty}^{2}+\Lambda\left(\frac{1}{c_{0}},|h(t)|_{\mathcal{H}^{3, \infty}}^{2}\right)\left(\varepsilon\|\nabla v(t)\|_{\mathcal{H}^{1, \infty}}^{2}+\varepsilon\|v(t)\|_{\mathcal{H}^{1, \infty}}^{2}\right) \\
& \leq \Lambda_{0} \varepsilon\left\|\nabla \omega_{\mathbf{n}}\right\|_{\infty}^{2}+\left\|\Delta^{\varphi} p\right\|_{\mathcal{H}^{1}}^{2}+\Lambda\left(\frac{1}{c_{0}}, \mathcal{Q}_{5}(t)\right)\left(1+\varepsilon\|\nabla p\|_{\mathcal{H}^{4}}^{2}\right)+\Lambda_{0}\left\|\omega_{\mathbf{n}}\right\|_{\mathcal{H}^{1, \infty}}^{2} \\
& \leq \Lambda_{0} \varepsilon\left\|\nabla \omega_{\mathbf{n}}\right\|_{\infty}^{2}+\Lambda_{0}\left\|\omega_{\mathbf{n}}\right\|_{\mathcal{H}^{1, \infty}}^{2}+\Lambda\left(\frac{1}{c_{0}}, Y_{m}(0)\right)+\Lambda_{0} \int_{0}^{t} \Lambda\left(Y_{m}(\tau)\right) d \tau
\end{aligned}
$$

Thus, it suffices to bound $\left\|\omega_{\mathbf{n}}\right\|_{\mathcal{H}^{1, \infty}}^{2}$ and $\varepsilon\left\|\nabla \omega_{\mathbf{n}}(t)\right\|_{\infty}^{2}$. However, the disadvantage of the term $\omega_{\mathbf{n}}$ is that it does not vanish on the boundary. But fortunately, the expression of $\omega_{\mathbf{n}}$ in (4.5.2) and the dynamic boundary condition (1.31) yield that, on the boundary

$$
\omega_{\mathbf{n}}^{b}=-2 \Pi\left\{\left(\nabla_{y}, 0\right)^{t} \partial_{t} h-\left(\nabla^{\varphi} \mathbf{N}\right)^{t} v^{b}\right\} .
$$

Thus, we introduce

$$
\zeta_{\mathbf{n}}:=\omega_{\mathbf{n}}+2 \Pi\left\{\left(\nabla_{y}, 0\right)^{t} \partial_{t} \eta-\left(\nabla^{\varphi} \mathbf{N}\right)^{t} v\right\}
$$

which vanishes on the boundary. Then, for $m \geq 6$, it follows from (4.6.2), (4.6.27) and (4.6.28) that

$$
\begin{aligned}
\left\|\omega_{\mathbf{n}}\right\|_{\mathcal{H}^{1, \infty}}^{2} & \leq\left\|\zeta_{\mathbf{n}}\right\|_{\mathcal{H}^{1, \infty}}^{2}+\Lambda\left(\frac{1}{c_{0}},|h(t)|_{\mathcal{H}^{3, \infty}}^{2}\right)\left(1+\|v(t)\|_{\mathcal{H}^{1, \infty}}^{2}\right) \leq\left\|\zeta_{\mathbf{n}}\right\|_{\mathcal{H}^{1, \infty}}^{2}+\Lambda\left(\frac{1}{c_{0}}, \mathcal{Q}_{5}(t)\right) \\
& \leq\left\|\zeta_{\mathbf{n}}\right\|_{\mathcal{W}^{1, \infty}}^{2}+\Lambda\left(\frac{1}{c_{0}}, Y_{m}(0)\right)+\Lambda_{0} \int_{0}^{t} \Lambda\left(Y_{m}(\tau)\right) d \tau
\end{aligned}
$$

and

$$
\begin{aligned}
\varepsilon\left\|\nabla \omega_{\mathbf{n}}\right\|_{\infty}^{2} & \leq \varepsilon\left\|\nabla \zeta_{\mathbf{n}}\right\|_{\infty}^{2}+\Lambda\left(\frac{1}{c_{0}},|h(t)|_{\mathcal{H}^{3, \infty}}^{2}\right) \varepsilon\left(1+\|\nabla v(t)\|_{\infty}^{2}\right) \\
& \leq \varepsilon\left\|\nabla \zeta_{\mathbf{n}}\right\|_{\infty}^{2}+\Lambda\left(\frac{1}{c_{0}}, Y_{m}(0)\right)+\Lambda_{0} \int_{0}^{t} P\left(Y_{m}(\tau)\right) d \tau .
\end{aligned}
$$


As a consequence, it remains to estimate $\left\|\zeta_{\mathbf{n}}(t)\right\|_{\mathcal{H}^{1, \infty}}^{2}$ and $\varepsilon\left\|\nabla \zeta_{\mathbf{n}}(t)\right\|_{\infty}^{2}$. Moreover, by using the following lemma, one can see that actually it suffices to derive these $L^{\infty}$-estimates near the boundary, that is $\left\|\chi \zeta_{\mathbf{n}}(t)\right\|_{\mathcal{W}^{1, \infty}}^{2}$ and $\varepsilon\left\|\chi \nabla \zeta_{\mathbf{n}}(t)\right\|_{\infty}^{2}$ with $\chi$ compact supported and equal to 1 in the vicinity of $z=0$.

Lemma 4.25 For any smooth cut-off function $\chi$ such that $\chi=0$ in a vicinity of $z=0$, it holds that for $m \geq k+2$,

$$
\|\chi f\|_{\mathcal{H}^{k, \infty}} \lesssim\|f\|_{\mathcal{H}^{m}} .
$$

This Lemma follows from the Sobolev embedding and the fact that the standard Sobolev norms are equivalent to the conormal ones away from the boundary. In fact, Lemma 4.25) and (4.6.2) imply, for $m \geq 6$, that

$$
\left\|\zeta_{\mathbf{n}}\right\|_{\mathcal{H}^{1, \infty}}^{2} \lesssim\left\|\chi \zeta_{\mathbf{n}}\right\|_{\mathcal{H}^{1, \infty}}^{2}+\|v\|_{\mathcal{H}^{2, \infty}}^{2} \lesssim\left\|\chi \zeta_{\mathbf{n}}\right\|_{\mathcal{H}^{1, \infty}}^{2}+\Lambda\left(\frac{1}{c_{0}}, Y_{m}(0)\right)+\int_{0}^{t} \Lambda\left(\frac{1}{c_{0}}, Y_{m}(\tau)\right) d \tau .
$$

One can obtains the following lemma by the same argument in [32] but using $\left\|\chi \zeta_{\mathbf{n}}\right\|_{\mathcal{H}^{1, \infty}}^{2}$ instead of $\left\|\chi S_{\mathbf{n}}\right\|_{\mathcal{H}^{1, \infty}}^{2}$ :

Lemma 4.26 For $m \geq 6$, it holds that

$$
\|\nabla v(t)\|_{\mathcal{H}^{1, \infty}}^{2} \leq \Lambda\left(\frac{1}{c_{0}}, Y_{m}(0)\right)+t \sup _{0 \leq \tau \leq t} \Lambda\left(\frac{1}{c_{0}}, Y_{m}(\tau)\right) \cdot\left(1+\varepsilon^{2}\left|\mathcal{Z}^{m} h(t)\right|_{\frac{1}{2}}^{2}+\int_{0}^{t}\|\nabla p(\tau)\|_{\mathcal{H}^{5}}^{2} d \tau\right) .
$$

Proof. As indicated by (4.6.26), (4.6.30), 4.6.33) and (4.6.36), we need only to estimate $\left\|\chi \zeta_{\mathbf{n}}\right\|_{\mathcal{H}^{1, \infty}}^{2}$. The main difficulty of this estimate is to handle the commutator between $\mathcal{Z}_{i}$ and $\Delta^{\varphi}$. We shall use a normal geodesic coordinate system in the vicinity of the boundary which gives a simpler expression of Laplacian 32 . Here, we can use this coordinate system because $L^{\infty}$-estimate does not require the highest regularity of the boundary. Let us define a new parametrization at the vicinity of the boundary

$$
\begin{aligned}
\Psi(t, \cdot): \mathcal{S}= & \mathbb{R}^{2} \times(-\infty, 0) \rightarrow D(t), \\
& (y, z) \mapsto(y, h(t, y))+z \mathbf{n}^{b}(t, y),
\end{aligned}
$$

where $D(t)$ is defined in (1.25) and $\mathbf{n}^{b}$ is the unit exterior normal $\mathbf{n}^{b}(t, y)=\left(-\partial_{1} h,-\partial_{2} h, 1\right) /|\mathbf{N}|$. Note that

$$
D \Psi(t, \cdot)=\left(\begin{array}{ccc}
1-z \partial_{1}\left(\frac{\partial_{1} h}{\mid \mathbf{N}}\right) & -z \partial_{2}\left(\frac{\partial_{1} h}{\mid \mathbf{N}} \mid\right) & -\frac{\partial_{1} h}{|\mathbf{N}|} \\
-z \partial_{1}\left(\frac{\partial_{2} h}{\mid \mathbf{N}} \mid\right) & 1-z \partial_{2}\left(\frac{\partial_{2} h}{\mid \mathbf{N}} \mid\right) & -\frac{\partial_{2} h}{|\mathbf{N}|} \\
\partial_{1} h+z \partial_{1}\left(\frac{1}{|\mathbf{N}|}\right) & \partial_{2} h+z \partial_{2}\left(\frac{1}{|\mathbf{N}|} \mid\right) & \left.\frac{1}{|\mathbf{N}|}\right)
\end{array}\right),
$$

which is of the form $M_{0}+R$ with $|R|_{\infty} \lesssim z|h|_{2, \infty}$ and

$$
M_{0}=\left(\begin{array}{ccc}
1 & 0 & -\frac{\partial_{1} h}{|\mathbf{N}|} \\
0 & 1 & -\frac{\partial_{2} h}{|\mathbf{N}|} \\
\partial_{1} h & \partial_{2} h & \left.\frac{1}{|\mathbf{N}|}\right)
\end{array}\right)
$$

is invertible. This yields that $\Psi(t, \cdot)$ is a diffeomorphism from $\mathbb{R}^{2} \times(-\delta, 0)$ to a vicinity of $\partial \Omega_{t}$ for some $\delta>0$ which depends only on $c_{0}>0$. By this parametrization, the scalar product in $\Omega_{t}$ induces a Riemman metric which is given by

$$
g(y, z)=\left(\begin{array}{cc}
\tilde{g}(y, z) & 0 \\
0 & 1
\end{array}\right)
$$

and the Laplacian in this coordinate system is of the form:

$$
\Delta_{g} f=\partial_{z z} f+\frac{1}{2} \partial_{z}(\ln |g|) \partial_{z} f+\Delta_{\tilde{g}} f
$$

where $|g|$ denotes the determinant of the matrix $g$ and $\Delta_{\tilde{g}} f$ is given by

$$
\Delta_{\tilde{g}} f=\frac{1}{|\tilde{g}|^{\frac{1}{2}}} \sum_{1 \leq i, j \leq 2} \partial_{y_{i}}\left(\tilde{g}^{i j}|g|^{\frac{1}{2}} \partial_{y_{j}} f\right),
$$


which involves only the tangential derivatives.

In order to use this normal geodesic coordinate system, one first localizes the equation for $\zeta_{\mathbf{n}}$. Set

$$
\omega^{\chi}=\chi(z) \omega
$$

where $\chi(z)$ is given as $\chi(z)=\tilde{\kappa}\left(\frac{z}{\delta\left(c_{0}\right)}\right)$ with $\tilde{\kappa}(z)$ be a smooth function of compact support such that $\tilde{\kappa}(z) \in[0,1]$ taking the value 1 in the vicinity of $z=0$, and $\delta\left(c_{0}\right)$ to be determined later. Note that this choice implies that $\left|\chi^{k}(z)\right| \leq \Lambda_{0}$.

Applying $\nabla^{\varphi} \times$ to $(1.31)_{2}$ yields that in $\mathcal{S}$,

$$
\varrho \partial_{t}^{\varphi} \omega+\varrho v \cdot \nabla^{\varphi} \omega-\mu \varepsilon \Delta^{\varphi} \omega=F_{\omega},
$$

where

$$
F_{\omega}=-\nabla^{\varphi} \varrho \times \partial_{t}^{\varphi} v-\nabla^{\varphi} \varrho \times\left(\left(v \cdot \nabla^{\varphi}\right) v\right)+\varrho \omega \cdot \nabla^{\varphi} v-\varrho \operatorname{div}^{\varphi} v \omega .
$$

Furthermore, it follows from 4.6.40) and 44.6.41) that

$$
\varrho \partial_{t}^{\varphi} \omega^{\chi}+\varrho v \cdot \nabla^{\varphi} \omega^{\chi}-\mu \varepsilon \Delta^{\varphi} \omega^{\chi}=F_{\omega \chi}
$$

where $F_{\omega \chi}:=F_{1}^{\chi}+\chi F_{\omega}$ with

$$
F_{1}^{\chi}=\varrho\left(V_{z} \partial_{z} \chi\right) \omega-\mu \varepsilon \nabla^{\varphi} \chi \cdot \nabla^{\varphi} \omega-\mu \varepsilon \Delta^{\varphi} \chi \omega .
$$

Since $F_{1}^{\chi}$ is supported away from the boundary, it follows from (3.22), (3.23) and (4.6.35) that

$$
\left\|F_{1}^{\chi}\right\|_{\mathcal{H}^{1, \infty}}^{2} \lesssim \Lambda\left(\frac{1}{c_{0}},|h(t)|_{\mathcal{H}^{1, \infty}}^{2}+\|(v, p)(t)\|_{\mathcal{H}^{1, \infty}}^{2}\right)\|v(t)\|_{\mathcal{H}^{5}}^{2} \lesssim \Lambda\left(\frac{1}{c_{0}}, \mathcal{Q}_{5}(t)\right) .
$$

Next, we define $\omega^{\Psi}:=\omega^{\chi}\left(t, \Phi^{-1}(t, \cdot) \circ \Psi\right)$. This change of variable is well-defined if we choose $\delta\left(c_{0}\right)$ small enough such that $D \Psi$ is invertible. Then, $\omega^{\Psi}$ solves in $\mathcal{S}$ the convection diffusion equations

$$
\varrho \partial_{t} \omega^{\Psi}+\varrho \mathbf{b} \cdot \nabla \omega^{\Psi}-\mu \varepsilon\left(\partial_{z z}+\frac{1}{2} \partial_{z}(\ln |g|) \partial_{z}\right) \omega^{\Psi}=F_{\omega \chi}\left(t, \Phi^{-1} \circ \Psi\right)+\mu \varepsilon \Delta_{\tilde{g}} \omega^{\Psi}
$$

where

$$
\mathbf{b}=(D \Psi)^{-1}\left(v\left(t, \Phi^{-1} \circ \Psi\right)-\partial_{t} \Psi\right)
$$

Set

$$
\omega_{\mathbf{n}}^{\Psi}(t, y, z)=\Pi^{b}(t, y)\left(\omega^{\Psi}(t, y, z) \times \mathbf{N}^{b}(t, y)\right),
$$

with $\mathbf{N}^{b}=\left(-\partial_{1} h,-\partial_{2} h, 1\right)$ and $\Pi^{b}=\operatorname{Id}-\mathbf{n}^{b} \otimes \mathbf{n}^{b}$. Note that $\Pi^{b}$ and $\mathbf{n}^{b}$ are independent of $z$. This yields that $\omega_{\mathbf{n}}^{\Psi}$ solves

$$
\varrho \partial_{t} \omega_{\mathbf{n}}^{\Psi}+\varrho \mathbf{b} \cdot \nabla \omega_{\mathbf{n}}^{\Psi}-\mu \varepsilon\left(\partial_{z z}+\frac{1}{2} \partial_{z}(\ln |g|) \partial_{z}\right) \omega_{\mathbf{n}}^{\Psi}=F_{\omega}^{\Psi}
$$

where $F_{\omega}^{\Psi}$ is given by

$$
F_{\omega}^{\Psi}=\Pi^{b} F_{\omega \chi} \times \mathbf{N}^{b}+F_{\omega}^{\Psi, 1}+F_{\omega}^{\Psi, 2},
$$

with

$$
\begin{aligned}
& F_{\omega}^{\Psi, 1}=\varrho\left(\partial_{t} \Pi^{b}+\mathbf{b}_{y} \cdot \nabla \Pi^{b}\right) \omega^{\Psi} \times \mathbf{N}^{b}+\varrho \Pi\left\{\omega^{\Psi} \cdot\left(\partial_{t}+\mathbf{b}_{y} \cdot \nabla_{y}\right) \mathbf{N}^{b}\right\} \\
& F_{\omega}^{\Psi, 2}=-\mu \varepsilon \Pi^{b}\left(\Delta_{\tilde{g}} \omega^{\Psi} \times \mathbf{N}^{b}\right)
\end{aligned}
$$

Define

$$
v^{\Psi}=(\chi v)\left(t, \Phi^{-1} \circ \Psi\right) .
$$

Note that $\nabla^{\varphi} \mathbf{N}^{b}=\nabla \mathbf{N}^{b}$ is independent of $z$. Thus $\Pi^{b}\left\{\left(\nabla^{\varphi} \mathbf{N}^{b}\right)^{t} v^{\Psi}\right\}$ solves

$$
\varrho \partial_{t} \Pi^{b}\left\{\left(\nabla^{\varphi} \mathbf{N}^{b}\right)^{t} v^{\Psi}\right\}+\varrho \mathbf{b} \cdot \nabla \Pi^{b}\left\{\left(\nabla^{\varphi} \mathbf{N}^{b}\right)^{t} v^{\Psi}\right\}-\mu \varepsilon\left(\partial_{z z}+\frac{1}{2} \partial_{z}(\ln |g|) \partial_{z}\right) \Pi^{b}\left\{\left(\nabla^{\varphi} \mathbf{N}^{b}\right)^{t} v^{\Psi}\right\}=F_{v}^{\Psi},
$$

where

$$
F_{v}^{\Psi}=\Pi^{b}\left\{\left(\nabla^{\varphi} \mathbf{N}^{b}\right)^{t}\left(\chi F_{v}+F_{2}^{\chi}\right)\right\}+F_{v}^{\Psi, 1}+F_{v}^{\Psi, 2},
$$


with

$$
\begin{aligned}
& F_{v}=-\nabla^{\varphi} p+(\mu+\lambda) \varepsilon \nabla^{\varphi} \operatorname{div}^{\varphi} v, \\
& F_{2}^{\chi}=\varrho\left(V_{z} \partial_{z} \chi\right) v-\mu \varepsilon \nabla \chi \cdot \nabla^{\varphi} v-\mu \varepsilon \Delta^{\varphi} \chi v, \\
& F_{v}^{\Psi, 1}=\varrho\left(\partial_{t} \Pi^{b}+\mathbf{b}_{y} \cdot \nabla \Pi^{b}\right)\left(\nabla^{\varphi} \mathbf{N}^{b}\right)^{t} v^{\Psi}+\varrho \Pi\left\{\left(\partial_{t}+\mathbf{b}_{y} \cdot \nabla_{y}\right)\left(\nabla^{\varphi} \mathbf{N}\right)^{t} v^{\Psi}\right\}, \\
& F_{v}^{\Psi, 2}=-\mu \varepsilon \Pi^{b}\left(\left(\nabla^{\varphi} \mathbf{N}^{b}\right)^{t} \Delta_{\tilde{g}} v^{\Psi}\right) .
\end{aligned}
$$

Set

$$
\zeta_{\mathbf{n}}^{\Psi}=\omega_{\mathbf{n}}^{\Psi}-2 \Pi^{b}\left\{\left(\nabla^{\varphi} \mathbf{N}^{b}\right)^{t} v^{\Psi}\right\}+2 \Pi^{b}\left(\left(\nabla_{y}, 0\right)^{t} \partial_{t} \eta\right) .
$$

It follows from (4.6.49) and (4.6.54) that $\zeta_{\mathbf{n}}^{\Psi}$ solves in $\mathcal{S}$

$$
\varrho \partial_{t} \zeta_{\mathbf{n}}^{\Psi}+\varrho \mathbf{b} \cdot \nabla \zeta_{\mathbf{n}}^{\Psi}-\mu \varepsilon\left(\partial_{z z}+\frac{1}{2} \partial_{z}(\ln |g|) \partial_{z}\right) \zeta_{\mathbf{n}}^{\Psi}=F_{\omega}^{\Psi}+F_{v}^{\Psi}+F_{\eta}^{\Psi},
$$

where

$$
F_{\eta}^{\Psi}=\varrho \partial_{t}\left(\Pi^{b}\left(\nabla_{y}, 0\right)^{t} \partial_{t} \eta^{\Psi}\right)+\varrho \mathbf{b} \cdot \nabla\left(\Pi^{b}\left(\nabla_{y}, 0\right)^{t} \partial_{t} \eta^{\Psi}\right)-\mu \varepsilon\left(\partial_{z z}+\frac{1}{2} \partial_{z}(\ln |g|) \partial_{z}\right)\left(\Pi^{b}\left(\nabla_{y}, 0\right)^{t} \partial_{t} \eta^{\Psi}\right),
$$

with $\eta^{\Psi}$ defined by $\chi \eta\left(t, \Phi^{-1} \circ \Psi\right)$. In order to eliminate the term $\frac{1}{2} \partial_{z}(\ln |g|) \partial_{z} \zeta_{\mathbf{n}}^{\Psi}$, we define

$$
\zeta_{\mathbf{n}}^{\Psi}=\frac{1}{|g|^{\frac{1}{4}}} \tilde{\zeta}_{\mathbf{n}}^{\Psi} .
$$

Plugging $\tilde{\zeta}_{\mathbf{n}}^{\Psi}$ into (4.6.61), one obtains that

$$
\varrho \partial_{t} \tilde{\zeta}_{\mathbf{n}}^{\Psi}+\varrho \mathbf{b} \cdot \nabla \tilde{\zeta}_{\mathbf{n}}^{\Psi}-\mu \varepsilon \partial_{z z} \tilde{\zeta}_{\mathbf{n}}^{\Psi}=|g|^{\frac{1}{4}}\left(F_{\omega}^{\Psi}+F_{v}^{\Psi}+F_{\eta}^{\Psi}+F_{g}^{\Psi}\right)=: S,
$$

where $F_{g}^{\Psi}$

$$
F_{g}^{\Psi}=-\left(\varrho \partial_{t}\left(|g|^{-\frac{1}{4}}\right)+\varrho \cdot \mathbf{b} \nabla\left(|g|^{-\frac{1}{4}}\right)-\mu \varepsilon \partial_{z z}\left(|g|^{-\frac{1}{4}}\right)-2 \mu \varepsilon \frac{\left(\partial_{z}|g|^{-\frac{1}{4}}\right)^{2}}{|g|^{-\frac{1}{4}}}\right) \tilde{\zeta}_{\mathbf{n}}^{\Psi} .
$$

Moreover, the boundary condition reads as

$$
\tilde{\zeta}_{\mathbf{n}}^{\Psi}=\zeta_{\mathbf{n}}^{\Psi}=\zeta_{\mathbf{n}}=0
$$

Since Sobolev conormal spaces are invariant by diffeomorphisms which preserve the boundary (see Lemma 9.5 in [32]) and $\left|\Pi-\Pi^{b}\right|+\left|\mathbf{N}-\mathbf{N}^{b}\right|=\mathcal{O}(z)$ in the vicinity of the boundary, one obtains that

$$
\begin{aligned}
\left\|\zeta_{\mathbf{n}}\right\|_{\mathcal{H}^{1, \infty}}^{2} & \leq \Lambda_{0}\left(\left\|\zeta_{\mathbf{n}}^{\Psi}\right\|_{\mathcal{H}^{1, \infty}}^{2}+|h(t)|_{\mathcal{H}^{3, \infty}}^{2}+\|v\|_{\mathcal{H}^{2, \infty}}^{2}\right) \leq \Lambda_{0}\left\|\tilde{\zeta}_{\mathbf{n}}^{\Psi}\right\|_{\mathcal{H}^{1, \infty}}^{2}+\Lambda\left(\frac{1}{c_{0}}, \mathcal{Q}_{5}(t)\right) \\
& \leq \Lambda_{0}\left\|\tilde{\zeta}_{\mathbf{n}}^{\Psi}\right\|_{\mathcal{H}^{1, \infty}}^{2}+\Lambda\left(Y_{m}(0)\right)+\int_{0}^{t} \Lambda\left(Y_{m}(\tau)\right) d \tau
\end{aligned}
$$

which implies that it suffices to estimate $\left\|\tilde{\zeta}_{\mathbf{n}}^{\Psi}\right\|_{\mathcal{H}^{1, \infty}}^{2}$. Now, we will estimate $\left\|\tilde{\zeta}_{\mathbf{n}}^{\Psi}\right\|_{\mathcal{H}^{1, \infty}}^{2}$ by taking advantage of (4.6.63) and (4.6.65). First, since

$$
b_{3}(t, y, 0)=\sum_{j=1}^{3}(D \Psi)_{3 j}^{-1}\left(v_{j}-\partial_{t} \Psi_{j}\right)(t, y, 0)=0,
$$

then (4.6.63) can be rewritten as

$$
\begin{aligned}
& \varrho(t, y, 0)\left(\partial_{t}+b_{1}(t, y, 0) \partial_{1}+b_{2}(t, y, 0) \partial_{2}+z \partial_{z} b_{3}(t, y, 0)\right) \tilde{\zeta}_{\mathbf{n}}^{\Psi}-\mu \varepsilon \partial_{z z} \tilde{\zeta}_{\mathbf{n}}^{\Psi} \\
= & S-[\varrho(t, y, z)-\varrho(t, y, 0)] \partial_{t} \tilde{\zeta}_{\mathbf{n}}^{\Psi}-\sum_{i=1,2} \varrho\left[b_{i}(t, y, z)-b_{i}(t, y, 0)\right] \partial_{i} \tilde{\zeta}_{\mathbf{n}}^{\Psi} \\
& -\varrho(t, y, 0)\left[b(t, y, z)-z \partial_{z} b_{3}(t, y, 0)\right] \partial_{z} \tilde{\zeta}_{\mathbf{n}}^{\Psi}-[\varrho(t, y, z)-\varrho(t, y, 0)] z \partial_{z} b_{3}(t, y, 0) \partial_{z} \tilde{\zeta}_{\mathbf{n}}^{\Psi}=: G .
\end{aligned}
$$


It follows from Lemma 6.2 in the Appendix that, for $m \geq 6$,

$$
\begin{aligned}
\left\|\tilde{\zeta}_{\mathbf{n}}^{\Psi}(t)\right\|_{\mathcal{H}^{1, \infty}} \lesssim & \left\|\tilde{\zeta}_{\mathbf{n}}^{\Psi}(0)\right\|_{\mathcal{H}^{1, \infty}}+\int_{0}^{t}\left\|\varrho^{-1}\right\|_{\infty}\|G\|_{\mathcal{H}^{1, \infty}} d \tau \\
& +\int_{0}^{t}\left(1+\left\|\varrho^{-1}\right\|_{\infty}\right)\left(1+\sum_{i=0}^{2}\left\|\mathcal{Z}_{i}\left(\varrho, b, \partial_{z} b_{3}\right)\right\|_{\infty}^{2}\right)\left\|\tilde{\zeta}_{\mathbf{n}}^{\Psi}\right\|_{\mathcal{H}^{1, \infty}} d \tau \\
\lesssim & \left\|\tilde{\zeta}_{\mathbf{n}}^{\Psi}(0)\right\|_{\mathcal{H}^{1, \infty}}+\Lambda_{0} \int_{0}^{t}\|G\|_{\mathcal{H}^{1, \infty}} d \tau+\int_{0}^{t} \Lambda\left(\frac{1}{c_{0}}, Y_{m}(\tau)\right) d \tau
\end{aligned}
$$

It remains to estimate $\int_{0}^{t}\|G\|_{\mathcal{H}^{1, \infty}} d \tau$. First, it follows from (4.6.42), (4.6.45) and (4.6.50)-(4.6.52) that

$$
\begin{aligned}
\left\||g|^{\frac{1}{4}} F_{\omega}^{\Psi}\right\|_{\mathcal{H}^{1, \infty}}^{2} & \leq \Lambda\left(\frac{1}{c_{0}}, \mathcal{Q}_{5}(t)\right)\left(\left(1+\|\nabla p\|_{\mathcal{H}^{1, \infty}}^{2}\right)\|\nabla v\|_{\mathcal{H}^{1, \infty}}^{2}+\|\nabla v\|_{\mathcal{H}^{1, \infty}}^{4}+\varepsilon^{2}\|\nabla v\|_{\mathcal{H}^{3, \infty}}^{2}\right) \\
& \leq \Lambda\left(\frac{1}{c_{0}}, Y_{m}(t)\right)\left(1+\varepsilon^{2}\left\|\nabla^{2} v\right\|_{\mathcal{H}^{4}}\|\nabla v\|_{\mathcal{H}^{5}}\right), m \geq 6
\end{aligned}
$$

where Lemma 4.23 has been used several times. Next, in a similar way, one can get from (4.6.55)-(4.6.59) and Lemma 4.23 that

$$
\begin{aligned}
& \left\||g|^{\frac{1}{4}} F_{v}^{\Psi}\right\|_{\mathcal{H}^{1, \infty}}^{2} \leq \Lambda\left(\frac{1}{c_{0}}, \mathcal{Q}_{5}(t)\right)\left[1+\|\nabla p\|_{\mathcal{H}^{1, \infty}}^{2}+\|\nabla v\|_{\mathcal{H}^{1, \infty}}^{2}+\varepsilon^{2}\left\|\nabla \operatorname{div}^{\varphi} v\right\|_{\mathcal{H}^{1, \infty}}^{2}+\varepsilon^{2}\|\nabla v\|_{\mathcal{H}^{3, \infty}}^{2}\right] \\
& \leq \varepsilon^{2}\left\|\Delta^{\varphi} p\right\|_{\mathcal{H}^{2}}^{2}+\Lambda\left(\frac{1}{c_{0}}, \mathcal{Q}_{6}(t)+\left\|\Delta^{\varphi} p\right\|_{\mathcal{H}^{1}}^{2}+\|\nabla v\|_{\mathcal{H}^{1, \infty}}^{2}\right)\left(\varepsilon^{2}\|\nabla p\|_{\mathcal{H}^{5}}^{2}+\varepsilon^{2}\|\nabla v\|_{\mathcal{H}^{3, \infty}}^{2}\right) \\
& \leq \Lambda\left(\frac{1}{c_{0}}, Y_{m}(t)\right)\left(1+\varepsilon^{2}\left\|\nabla^{2} v\right\|_{\mathcal{H}^{4}}\|\nabla v\|_{\mathcal{H}^{5}}\right), m \geq 6
\end{aligned}
$$

It follows from (4.6.62), (4.6.64) and Lemma 4.23 that

$$
\left\||g|^{\frac{1}{4}} F_{\eta}^{\Psi}\right\|_{\mathcal{H}^{1, \infty}}^{2}+\left\||g|^{\frac{1}{4}} F_{g}^{\Psi}\right\|_{\mathcal{H}^{1, \infty}}^{2} \leq \Lambda\left(\frac{1}{c_{0}}, Y_{m}(t)\right)\left(1+\varepsilon^{2}\left|\mathcal{Z}^{m} h(t)\right|_{\frac{1}{2}}^{2}\right), m \geq 6 .
$$

Therefore, combining (4.6.70)-(4.6.72) leads to that

$$
\|S\|_{\mathcal{H}^{1, \infty}}^{2} \leq \Lambda\left(\frac{1}{c_{0}}, Y_{m}(t)\right)\left(1+\varepsilon^{2}\left|\mathcal{Z}^{m} h(t)\right|_{\frac{1}{2}}^{2}+\varepsilon^{2}\left\|\nabla^{2} v\right\|_{\mathcal{H}^{4}}\|\nabla v\|_{\mathcal{H}^{5}}\right), m \geq 6
$$

Next, using the Taylor's formula and the fact that $\tilde{\zeta}$ is compactly supported in $z$, one obtains that

$$
\begin{aligned}
& \left\|(\varrho(t, y, z)-\varrho(t, y, 0)) \partial_{t} \tilde{\zeta}_{\mathbf{n}}^{\Psi}\right\|_{\mathcal{H}^{1, \infty}}^{2} \\
\lesssim & \Lambda_{0}\left\|\tilde{\zeta}_{\mathbf{n}}^{\Psi}\right\|_{\mathcal{H}^{1, \infty}}^{2}+\|\mathcal{Z}(\varrho(t, y, z)-\varrho(t, y, 0))\|_{\infty}^{2}\left\|\partial_{t} \tilde{\zeta}_{\mathbf{n}}^{\Psi}\right\|_{\infty}^{2}+\left\|(\varrho(t, y, z)-\varrho(t, y, 0)) \mathcal{Z} \partial_{t} \tilde{\zeta}_{\mathbf{n}}^{\Psi}\right\|_{\infty}^{2} \\
\leq & \Lambda_{0}\left\|\tilde{\zeta}_{\mathbf{n}}^{\Psi}\right\|_{\mathcal{H}^{1, \infty}}^{2}+\|\mathcal{Z} \varrho\|_{\infty}^{2}\left\|\tilde{\zeta}_{\mathbf{n}}^{\Psi}\right\|_{\infty}^{2}+\left\|\partial_{z} \varrho\right\|_{\infty}^{2}\left\|\frac{z}{1-z} \mathcal{Z} \partial_{t} \tilde{\zeta}_{\mathbf{n}}^{\Psi}\right\|_{\infty}^{2} .
\end{aligned}
$$

Due to (2.4), the following inequality holds for $|\alpha| \geq 0$

$$
\left\|\frac{z}{1-z} \mathcal{Z}^{\alpha} \tilde{\zeta}_{\mathbf{n}}^{\Psi}\right\|_{\infty}^{2} \lesssim\left\|\nabla\left(\frac{z}{1-z} \mathcal{Z}^{\alpha} \tilde{\zeta}_{\mathbf{n}}^{\Psi}\right)\right\|_{1}\left\|\frac{z}{1-z} \mathcal{Z}^{\alpha} \tilde{\zeta}_{\mathbf{n}}^{\Psi}\right\|_{2} \lesssim\left\|\mathcal{Z}^{\alpha} \tilde{\zeta}_{\mathbf{n}}^{\Psi}\right\|_{2}^{2}
$$

Therefore, plugging (4.6.75) with $|\alpha|=2$ into (4.6.74), one gets from (4.6.8) that

$$
\left\|(\varrho(t, y, z)-\varrho(t, y, 0)) \partial_{t} \tilde{\zeta}_{\mathbf{n}}^{\Psi}\right\|_{\mathcal{H}^{1, \infty}} \leq \Lambda\left(\frac{1}{c_{0}}, Y_{m}(t)\right), m \geq 6 .
$$

Similarly,

$$
\sum_{i=1,2}\left\|\varrho\left(b_{i}(t, y, z)-b_{i}(t, y, 0)\right) \partial_{i} \tilde{\zeta}_{\mathbf{n}}^{\Psi}\right\|_{\mathcal{H}^{1, \infty}}^{2} \leq \Lambda\left(\frac{1}{c_{0}}, Y_{m}(t)\right), m \geq 6
$$


Since $b_{3}(t, y, 0)=0$, it holds that

$$
\begin{aligned}
& \quad\left\|\varrho(t, y, 0)\left(b_{3}(t, y, z)-z \partial_{z} b_{3}(t, y, 0)\right) \partial_{z} \tilde{\zeta}_{\mathbf{n}}^{\Psi}\right\|_{\mathcal{H}^{1, \infty}}^{2} \\
& \leq\|\varrho\|_{\mathcal{W}^{1, \infty}}^{2}\left(\left\|\nabla b_{3}\right\|_{\infty}^{2}\left\|\tilde{\zeta}_{\mathbf{n}}^{\Psi}\right\|_{\mathcal{H}^{1, \infty}}^{2}+\left\|\mathcal{Z}\left(b_{3}(t, y, z)-z \partial_{z} b_{3}(t, y, 0)\right) \partial_{z} \tilde{\zeta}_{\mathbf{n}}^{\Psi}\right\|_{\infty}^{2}\right. \\
& \left.\quad+\left\|\left(b_{3}(t, y, z)-z \partial_{z} b_{3}(t, y, 0)\right) \mathcal{Z} \partial_{z} \tilde{\zeta}_{\mathbf{n}}^{\Psi}\right\|_{\infty}^{2}\right) \\
& \leq \Lambda\left(\frac{1}{c_{0}}, \mathcal{Q}_{5}(t)\right)\left(1+\|\nabla v\|_{\mathcal{H}^{1, \infty}}^{2}\right)\left\|\tilde{\zeta}_{\mathbf{n}}^{\Psi}\right\|_{\mathcal{H}^{1, \infty}}^{2}+\Lambda\left(\frac{1}{c_{0}}, \mathcal{Q}_{5}(t)\right)\left\|\partial_{z z} b_{3}\right\|_{\infty}^{2}\left\|\frac{z^{2}}{(1-z)^{2}} \partial_{z} \mathcal{Z} \tilde{\zeta}_{\mathbf{n}}^{\Psi}\right\|_{\infty}^{2} \\
& \leq \Lambda\left(\frac{1}{c_{0}}, \mathcal{Q}_{5}(t)\right)\left(1+\|\nabla v\|_{\mathcal{H}^{1, \infty}}^{2}\right)\left\|\tilde{\zeta}_{\mathbf{n}}^{\Psi}\right\|_{\mathcal{H}^{1, \infty}}^{2} \\
& \quad+\Lambda\left(\frac{1}{c_{0}}, \mathcal{Q}_{5}(t)\right)\left\|\partial_{z z} b_{3}\right\|_{\infty}^{2}\left\|\frac{z^{2}}{(1-z)^{2}} \partial_{z} \mathcal{Z} \tilde{\zeta}_{\mathbf{n}}^{\Psi}\right\|_{\mathcal{H}^{2}}\left\|\partial_{z}\left(\frac{z^{2}}{(1-z)^{2}} \partial_{z} \mathcal{Z} \tilde{\zeta}_{\mathbf{n}}^{\Psi}\right)\right\|_{\mathcal{H}^{1}} \\
& \leq \Lambda\left(\frac{1}{c_{0}}, Y_{m}(t)\right)+\Lambda\left(\frac{1}{c_{0}}, Y_{m}(t)\right)\left\|\partial_{z z} b_{3}\right\|_{\infty}^{2}\left\|\tilde{\zeta}_{\mathbf{n}}^{\Psi}\right\|_{\mathcal{H}^{4}}^{2}, \text { for } m \geq 6 .
\end{aligned}
$$

It follows from (4.6.39) and 4.6.47) that

$$
\begin{aligned}
& \left\|\partial_{z z} b_{3}\right\|_{\infty}^{2} \leq\left\|\partial_{z z} v \cdot \mathbf{N}\right\|_{\infty}^{2}+\Lambda\left(\frac{1}{c_{0}},|h|_{\mathcal{H}^{4, \infty}}^{2}\right) \\
& \lesssim \Lambda_{0}\left\|\partial_{z} \operatorname{div}^{\varphi} v\right\|_{\infty}^{2}+\Lambda_{0}\|\nabla v(t)\|_{\infty}^{2}+\Lambda\left(\frac{1}{c_{0}},|h|_{\mathcal{H}^{4, \infty}}^{2}\right) \leq \Lambda\left(\frac{1}{c_{0}}, Y_{m}(t)\right), m \geq 6
\end{aligned}
$$

where one has used that

$$
\begin{aligned}
\partial_{z z}(v \cdot \mathbf{N}) & =\partial_{z}\left(\partial_{z} v \cdot \mathbf{N}\right)+\partial_{z}\left(v \cdot \partial_{z} \mathbf{N}\right) \\
& =\partial_{z}\left(\partial_{z} \varphi\left(\operatorname{div}^{\varphi} v-\partial_{1} v_{1}-\partial_{2} v_{2}\right)\right)+\partial_{z}\left(v \cdot \partial_{z} \mathbf{N}\right)
\end{aligned}
$$

Thus,

$$
\left\|\varrho(t, y, 0)\left(b_{3}(t, y, z)-z \partial_{z} b_{3}(t, y, 0)\right) \partial_{z} \tilde{\zeta}_{\mathbf{n}}^{\Psi}\right\|_{\mathcal{H}^{1, \infty}}^{2} \leq \Lambda\left(\frac{1}{c_{0}}, Y_{m}(t)\right), m \geq 6 .
$$

Similarly, note that $(\varrho(t, y, z)-\varrho(t, y, 0)) z=\mathcal{O}\left(z^{2}\right)$ in a vicinity of the boundary, one gets that

$$
\left\|(\varrho(t, y, z)-\varrho(t, y, 0)) z \partial_{z} b_{3}(t, y, 0) \partial_{z} \tilde{\zeta}_{\mathbf{n}}^{\Psi}\right\|_{\mathcal{H}^{1, \infty}}^{2} \leq \Lambda\left(\frac{1}{c_{0}}, Y_{m}(t)\right), m \geq 6 .
$$

Thanks to (4.6.73), 4.6.76), 4.6.77), 4.6.80 and (4.6.81), one has that

$$
\|G\|_{\mathcal{H}^{1, \infty}}^{2} \leq \Lambda\left(\frac{1}{c_{0}}, Y_{m}(t)\right)\left(1+\varepsilon^{2}\left|\mathcal{Z}^{m} h(t)\right|_{\frac{1}{2}}^{2}+\varepsilon^{2}\left\|\nabla^{2} v\right\|_{\mathcal{H}^{4}}^{2}\right), m \geq 6 .
$$

Substituting (4.6.82) into (4.6.69) and using (4.3.31), one gets that

$$
\left\|\tilde{\zeta}_{\mathbf{n}}^{\Psi}(t)\right\|_{\mathcal{H}^{1, \infty}}^{2} \leq\left\|\tilde{\zeta}_{\mathbf{n}}^{\Psi}(0)\right\|_{\mathcal{H}^{1, \infty}}^{2}+\int_{0}^{t} \Lambda\left(\frac{1}{c_{0}}, Y_{m}(t)\right)\left(1+\varepsilon^{2}\left|\mathcal{Z}^{m} h(t)\right|_{\frac{1}{2}}^{2}\right) d \tau, m \geq 6 .
$$

Then, (4.6.37) follows from (4.6.26), 4.6.30), (4.6.33), (4.6.36), 44.6.66) and (4.6.83). Thus, the proof of this lemma is completed.

Lemma 4.27 For $m \geq 6$, it holds that

$$
\begin{aligned}
\varepsilon\left\|\partial_{z z} v(t)\right\|_{\infty}^{2} \leq t \sup _{0 \leq \tau \leq t} \Lambda\left(\frac{1}{c_{0}}, Y_{m}(\tau)+\varepsilon^{2}\left|\mathcal{Z}^{m} h(\tau)\right|_{\frac{1}{2}}^{2}\right) \cdot\left(1+\int_{0}^{t}\|\nabla p(\tau)\|_{\mathcal{H}^{5}}^{2} d \tau\right) \\
+\Lambda\left(\frac{1}{c_{0}}, Y_{m}(0)\right) .
\end{aligned}
$$

Proof. We shall reduce the problem to the estimate of $\varepsilon\left\|\partial_{z} \tilde{\zeta}_{\mathbf{n}}^{\Psi}\right\|_{\infty}$. First, it follows from (4.6.29), (4.6.31), (4.6.34) and (4.6.37) that

$$
\begin{gathered}
\varepsilon\left\|\partial_{z z} v\right\|_{\infty}^{2} \leq t \sup _{0 \leq \tau \leq t} \Lambda\left(\frac{1}{c_{0}}, Y_{m}(\tau)\right)\left(1+\varepsilon^{2}\left|\mathcal{Z}^{m} h(t)\right|_{\frac{1}{2}}^{2}+\int_{0}^{t}\|\nabla p(\tau)\|_{\mathcal{H}^{5}}^{2} d \tau\right) \\
+\Lambda\left(\frac{1}{c_{0}}, Y_{m}(0)\right)+\Lambda_{0} \varepsilon\left\|\nabla \zeta_{\mathbf{n}}\right\|_{\infty}^{2}, m \geq 6 .
\end{gathered}
$$


Note that $\left|\Pi-\Pi^{b}\right|+\left|\mathbf{N}-\mathbf{N}^{b}\right|=\mathcal{O}(z)$ in the vicinity of the boundary, one gets from the definitions of $\tilde{\zeta}_{\mathbf{n}}^{\Psi}$ and $\zeta_{\mathbf{n}}$ that

$$
\begin{aligned}
& \varepsilon\left\|\partial_{z} \zeta_{\mathbf{n}}(t)\right\|_{\infty}^{2} \leq \Lambda_{0}\left(\varepsilon\left\|\partial_{z} \tilde{\zeta}_{\mathbf{n}}^{\Psi}\right\|_{\infty}^{2}+\|(v, \nabla v)\|_{\mathcal{H}^{1, \infty}}^{2}\right) \\
& \leq \Lambda\left(\frac{1}{c_{0}}, Y_{m}(0)\right)+\Lambda_{0} t \sup _{0 \leq \tau \leq t} \Lambda\left(\frac{1}{c_{0}}, Y_{m}(\tau)\right)\left(1+\varepsilon^{2}\left|\mathcal{Z}^{m} h(t)\right|_{\frac{1}{2}}^{2}+\int_{0}^{t}\|\nabla p(\tau)\|_{\mathcal{H}^{5}}^{2} d \tau\right) \\
& \quad+\Lambda_{0} \varepsilon\left\|\partial_{z} \tilde{\zeta}_{\mathbf{n}}^{\Psi}\right\|_{\infty}, m \geq 6 .
\end{aligned}
$$

It remains to estimate $\varepsilon\left\|\partial_{z} \tilde{\zeta}_{\mathbf{n}}^{\Psi}\right\|_{\infty}^{2}$. Since $\tilde{\zeta}_{\mathbf{n}}^{\Psi}$ solves (4.6.63) in $\mathcal{S}$ with the homogeneous Dirichlet boundary condition, one can use the one-dimensional heat kernel of $z>0$

$$
G\left(t, z, z^{\prime}\right)=\frac{1}{\sqrt{4 \pi \mu \varepsilon t}}\left(\exp \left(-\frac{\left(z-z^{\prime}\right)^{2}}{4 \mu \varepsilon t}\right)-\exp \left(-\frac{\left(z+z^{\prime}\right)^{2}}{4 \mu \varepsilon t}\right)\right)
$$

to obtain

$$
\begin{aligned}
\sqrt{\varepsilon} \partial_{z} \tilde{\zeta}_{\mathbf{n}}^{\Psi}= & \sqrt{\varepsilon} \int_{0}^{+\infty} \partial_{z} G\left(t, z, z^{\prime}\right) \tilde{\zeta}_{\mathbf{n}}^{\Psi}(0) d z^{\prime} \\
& +\int_{0}^{t} \int_{0}^{+\infty} \sqrt{\varepsilon} \partial_{z} G\left(t-\tau, z, z^{\prime}\right)\left(S-\rho \mathbf{b} \cdot \nabla \tilde{\zeta}_{\mathbf{n}}^{\Psi}-(\rho-1) \partial_{t} \tilde{\zeta}_{\mathbf{n}}^{\Psi}\right) d z^{\prime} d \tau
\end{aligned}
$$

It follows from $\tilde{\zeta}_{\mathbf{n}}^{\Psi}(0)=0$ and integration by parts that

$$
\sqrt{\varepsilon}\left\|\partial_{z} \tilde{\zeta}_{\mathbf{n}}^{\Psi}\right\|_{\infty} \leq \sqrt{\varepsilon}\left\|\partial_{z} \tilde{\zeta}_{\mathbf{n}}^{\Psi}(0)\right\|_{\infty}+\int_{0}^{t} \frac{1}{\sqrt{t-\tau}}\left(\|S\|_{\infty}+\left\|\varrho \mathbf{b} \cdot \nabla \tilde{\zeta}_{\mathbf{n}}^{\Psi}\right\|_{\infty}+\left\|(\varrho-1) \partial_{t} \tilde{\zeta}_{\mathbf{n}}^{\Psi}\right\|_{\infty}\right) .
$$

It then follows from (4.6.73) that

$$
\begin{aligned}
& \left(\int_{0}^{t} \frac{1}{\sqrt{t-\tau}}\|S\|_{\mathcal{H}^{1, \infty}} d \tau\right)^{2} \\
& \leq\left(\int_{0}^{t} \frac{1}{\sqrt{t-\tau}} \Lambda\left(\frac{1}{c_{0}}, Y_{m}(t)\right)\left[1+\varepsilon\left|\mathcal{Z}^{m} h(t)\right|_{\frac{1}{2}}+\sqrt{\varepsilon}\left\|\nabla^{2} v\right\|_{\mathcal{H}^{4}}^{\frac{1}{2}} \sqrt{\varepsilon}\|\nabla v\|_{\mathcal{H}^{5}}^{\frac{1}{2}}\right] d \tau\right)^{2} \\
& \leq t \Lambda\left(\frac{1}{c_{0}}, Y_{m}(t)+\varepsilon^{2}\left|\mathcal{Z}^{m} h(t)\right|_{\frac{1}{2}}^{2}\right)+\left(\int_{0}^{t} \varepsilon^{2}\left\|\nabla^{2} v\right\|_{\mathcal{H}^{4}}^{2}\right)^{\frac{1}{2}} t^{\frac{1}{2}} \Lambda\left(\frac{1}{c_{0}}, Y_{m}(t)\right) \\
& \leq t \Lambda\left(\frac{1}{c_{0}}, Y_{m}(t)+\varepsilon^{2}\left|\mathcal{Z}^{m} h(t)\right|_{\frac{1}{2}}^{2}\right)
\end{aligned}
$$

where one has used (4.3.31) in the last inequality. Since $b_{3}(t, y, 0)=0$, so

$$
\left\|\varrho \mathbf{b} \cdot \nabla \tilde{\zeta}_{\mathbf{n}}^{\Psi}\right\|_{\infty}^{2} \leq \Lambda_{0}\left(\|b\|_{\infty}^{2}+\|\nabla b\|_{\infty}^{2}\right)\left\|\tilde{\zeta}_{\mathbf{n}}^{\Psi}\right\|_{\mathcal{H}^{1, \infty}}^{2} \leq \Lambda\left(\frac{1}{c_{0}}, Y_{m}(t)\right) .
$$

Finally, it is easy to obtain that

$$
\left\|(\varrho-1) \partial_{t} \tilde{\zeta}_{\mathbf{n}}^{\Psi}\right\|_{\infty}^{2} \leq \Lambda\left(\frac{1}{c_{0}}, Y_{m}(t) .\right.
$$

Substituting 4.6.89)-44.6.91) into (4.6.88), one gets that

$$
\varepsilon\left\|\partial_{z} \tilde{\zeta}_{\mathbf{n}}^{\Psi}\right\|_{\infty}^{2} \leq \sqrt{\varepsilon}\left\|\partial_{z} \tilde{\zeta}_{\mathbf{n}}^{\Psi}(0)\right\|_{\infty}^{2}+t \Lambda\left(\frac{1}{c_{0}}, Y_{m}(t)+\varepsilon^{2}\left|\mathcal{Z}^{m} h(t)\right|_{\frac{1}{2}}^{2}\right) .
$$

Now (4.6.84) follows from (4.6.92), 44.6.85) and (4.6.86). Therefore, the proof of this lemma is completed. $\square$

\subsection{Proof of Theorem 4.1}

Based on the estimates obtained so far, we can complete the proof of Theorem4.1 in this subsection.First, (4.0.1) follows easily from conservation of mass. 
In order to prove (4.0.2), one chooses $A>0$ large so that

$$
\partial_{z} \varphi(0, y, z) \geq 1
$$

where $A$ depends only on the initial data $|h(0)|_{H^{3}}$. For two parameters $R>0$ and $c_{0}$ to be chosen $0<\frac{1}{c_{0}} \ll R$, define

$$
\begin{gathered}
T_{\star}^{\varepsilon}=\sup \left\{T \in\left[0, \frac{d_{0}}{8(1+R)}\right] ; \Theta_{m}(T) \leq R, \partial_{z} \varphi(t) \geq c_{0},|h|_{\mathcal{H}^{3, \infty}}+\left|\nabla_{y} h\right|_{\mathcal{H}^{\left[\frac{m}{2}\right]+1}} \leq \frac{1}{c_{0}},\right. \\
\left.-\left.\partial_{z}^{\varphi} p\right|_{z=0} \geq \frac{c_{0}}{2}, 0<\frac{1}{4 C_{0}} \leq \varrho(t) \leq 4 C_{0}, \forall t \in[0, T]\right\} .
\end{gathered}
$$

The restriction $T \leq \frac{d_{0}}{8(1+R)}$ guarantees that the fluid moves a distance no more than $\frac{1}{8} d_{0}$. Then $\tilde{\Omega}_{0,1}$, $\tilde{\Omega}_{k, \frac{1}{8}}, k=1, \cdots, n$ is still a valid covering of $\Omega_{t}$ with $t \leq T$.

Now define

$$
\begin{aligned}
& \mathcal{N}_{m}(t) \triangleq \mathcal{N}_{m}(p, v, h)(t) \\
& =\sup _{0 \leq \tau \leq t}\left\{1+\left\|\left(V^{m}, Q^{m}\right)(\tau)\right\|^{2}+\left|\left(h, \sqrt{\sigma} \nabla_{y} h\right)(\tau)\right|_{\mathcal{H}^{m}}^{2}+\|(\nabla p, \nabla v)(\tau)\|_{\mathcal{H}^{m-2}}^{2}\right. \\
& +\left\|\Delta^{\varphi} p(\tau)\right\|_{\mathcal{H}^{1}}^{2}+\|\nabla(p, v)(\tau)\|_{\mathcal{H}^{1, \infty}}^{2}+\varepsilon\left\|\left(\operatorname{div}^{\varphi} v, \nabla^{\varphi} p, S_{\mathbf{n}}\right)(\tau)\right\|_{\mathcal{H}^{m-1}}^{2}+\varepsilon\left|\mathcal{Z}^{m} h\right|_{\frac{1}{2}}^{2}+\varepsilon\left\|\nabla^{2} v(\tau)\right\|_{\mathcal{H}^{1, \infty}}^{2} \\
& \left.+\varepsilon\|\nabla(p, v)(\tau)\|_{\mathcal{H}^{m-1}}^{2}+\varepsilon\left\|\Delta^{\varphi} p(\tau)\right\|_{\mathcal{H}^{2}}^{2}\right\}+\int_{0}^{t}\|\nabla p(\tau)\|_{\mathcal{H}^{m-1}}^{2} d \tau+\left(\int_{0}^{t}\|\nabla v(\tau)\|_{\mathcal{H}^{m-1}}^{4} d \tau\right)^{\frac{1}{2}} \\
& +\varepsilon \int_{0}^{t}\|\nabla v(\tau)\|_{\mathcal{H}^{m}}^{2} d \tau+\varepsilon \int_{0}^{t}\left\|\nabla^{2} v(\tau)\right\|_{\mathcal{H}^{m-2}}^{2} d \tau+\varepsilon^{2} \int_{0}^{t}\left\|\nabla^{2} v(\tau)\right\|_{\mathcal{H}^{m-1}}^{2} d \tau
\end{aligned}
$$

which implies immediately that

$$
Y_{m}(t)+M(t) \leq \Lambda_{0} \Lambda\left(\mathcal{N}_{m}(t)\right), \mathcal{N}_{m}(t) \leq \Lambda_{0} \Lambda\left(\Theta_{m}(t)\right) \text { and } \Theta_{m}(t) \leq \Lambda_{0} \Lambda\left(\mathcal{N}_{m}(t)\right) .
$$

It follows from Propositions 4.17, 4.16 that

$$
\begin{aligned}
& \left\|\left(V^{m}, Q^{m}\right)(t)\right\|^{2}+\left|\left(h, \sqrt{\sigma} \nabla_{y} h\right)(t)\right|_{\mathcal{H}^{m}}^{2}+\left\|\left(\operatorname{div}^{\varphi} v, \nabla^{\varphi} p, S_{\mathbf{n}}\right)(t)\right\|_{\mathcal{H}^{m-2}}^{2}+\varepsilon\left\|\left(\operatorname{div}^{\varphi} v, \nabla^{\varphi} p, S_{\mathbf{n}}\right)(t)\right\|_{\mathcal{H}^{m-1}}^{2} \\
& +\varepsilon\left|\mathcal{Z}^{m} h\right|_{\frac{1}{2}}^{2}+\int_{0}^{t}\|\nabla p\|_{\mathcal{H}^{m-1}}^{2} d \tau+\left(\int_{0}^{t}\|\nabla v\|_{\mathcal{H}^{m-1}}^{4} d \tau\right)^{\frac{1}{2}}+\varepsilon \int_{0}^{t}\|\nabla v(\tau)\|_{\mathcal{H}^{m}}^{2}+\left\|\nabla^{2} v(\tau)\right\|_{\mathcal{H}^{m-2}}^{2} d \tau \\
& +\varepsilon^{2} \int_{0}^{t}\left\|\nabla^{2} v(\tau)\right\|_{\mathcal{H}^{m-1}}^{2} d \tau \leq \Lambda_{0} \Lambda\left(\mathcal{N}_{m}(0)+\|\nabla v(0)\|_{\mathcal{H}^{m-1}}^{2}\right)+t^{\frac{1}{2}} \Lambda_{0} \Lambda\left(\mathcal{N}_{m}(t)\right)
\end{aligned}
$$

Then, as a consequence of 4.7.5), Lemmas 4.24, 4.26 and 4.27, one obtains that

$$
\mathcal{N}_{m}(t) \leq \Lambda\left(C_{0}, \mathcal{N}_{m}(0)+\|\nabla v(0)\|_{\mathcal{H}^{m-1}}^{2}\right)+t^{\frac{1}{2}} \Lambda\left(C_{0}, \mathcal{N}_{m}(t)\right), \quad \forall t \in\left[0, T^{\varepsilon}\right] .
$$

Therefore,

$$
\Theta_{m}(t) \leq \Lambda\left(C_{0}, \Theta_{m}(0)+\|\nabla v(0)\|_{\mathcal{H}^{m-1}}^{2}\right)+t^{\frac{1}{2}} \Lambda\left(C_{0}, R\right), \quad \forall t \in\left[0, T^{\varepsilon}\right]
$$

Moreover,

$$
\begin{gathered}
\frac{1}{C_{0}} \exp (T \Lambda(R)) \leq \varrho(t) \leq C_{0} \exp (T \Lambda(R)), \forall t \in[0, T], \\
|h|_{\mathcal{H}^{3, \infty}}^{2}+\left|\nabla_{y} h\right|_{\mathcal{H}^{\left[\frac{m}{2}\right]+1}}^{2} \leq|h(0)|_{\mathcal{H}^{3, \infty}}^{2}+\left|\nabla_{y} h(0)\right|_{\mathcal{H}^{\left[\frac{m}{2}\right]+1}}^{2}+T \Lambda(R), \forall t \in[0, T], \\
\partial_{z} \varphi(t) \geq 1-\int_{0}^{t}\left\|\partial_{t} \nabla \eta(t)\right\|_{L^{\infty}} d \tau \geq 1-T \Lambda(R), \forall t \in[0, T],
\end{gathered}
$$

once the choice of $A>0$ so that (4.7.1) is satisfied. Finally, note that

$$
-\partial_{z}^{\varphi} p(t) \geq-\partial_{z}^{\varphi} p_{0}-\int_{0}^{t}\left\|\partial_{t} \partial_{z}^{\varphi} p(\tau)\right\| d \tau \geq-\partial_{z}^{\varphi} p_{0}-T \Lambda(R), \forall t \in[0, T] .
$$


Taking $R=2 \Lambda\left(C_{0}, \Theta_{m}(0)+\|\nabla v(0)\|_{\mathcal{H}^{m-1}}^{2}\right)$, in view of (4.7.7)-44.7.11), one gets that there exists $T_{*}>0$ depending only on $C_{0}, d_{0}$ and the initial data $\Theta_{m}(0)+\|\nabla v(0)\|_{\mathcal{H}^{m-1}}^{2}$ (hence independent of $\varepsilon$ and $\sigma$ ) such that for $T \leq \min \left(T_{*}, T^{\varepsilon}\right)$

$$
\left\{\begin{array}{l}
\Theta_{m}(t) \leq 2 \Lambda\left(C_{0}, \Theta_{m}(0)+\|\nabla v(0)\|_{\mathcal{H}^{m-1}}^{2}\right), \\
\partial_{z} \varphi(t) \geq 2 c_{0},|h(t)|_{\mathcal{H}^{3, \infty}}+\left|\nabla_{y} h(t)\right|_{\left.\mathcal{H}^{\left[\frac{m}{2}\right.}\right]+1} \leq \frac{1}{2 c_{0}}, \quad \forall t \in[0, T] . \\
-\left.\partial_{z}^{\varphi} p\right|_{z=0} \geq \frac{3 c_{0}}{4}, \quad 0<\frac{1}{2 C_{0}} \leq \varrho(t) \leq 2 C_{0},
\end{array}\right.
$$

Of course, it holds that $T_{*} \leq T_{\star}^{\varepsilon}$. Then taking $T_{a}=T_{*}$, one obtains (4.0.2) and closes the a priori assumption (4.0.4). Therefore, the proof Theorem 4.1 is completed.

\section{Proof of the Main Results}

Proof of Theorem 1.5. We shall combine the a priori estimates obtained to prove the uniform existence result. Fix $m \geq 6$, consider initial data $\left(p_{0}^{\varepsilon}, v_{0}^{\varepsilon}, h_{0}^{\varepsilon}\right) \in X_{N S}^{\varepsilon, m}$ such that

$$
\mathcal{I}_{m}(0):=\sup _{\varepsilon \in(0,1], \sigma \in[0,1]}\left(\left\|\left(p_{0}^{\varepsilon}, v_{0}^{\varepsilon}, h_{0}^{\varepsilon}\right)\right\|_{X_{m}^{\varepsilon}}^{2}+\left\|\nabla v_{0}^{\varepsilon}\right\|_{\mathcal{H}^{m-1}}^{2}\right) \leq \tilde{C}_{0}
$$

and

$$
0<\frac{1}{C_{0}} \leq \varrho_{0}^{\varepsilon} \leq C_{0}
$$

For such initial data, we are not aware of a local well-posedness result for (1.1) and (1.5) and (1.6). Thus, we shall prove the local existence by using energy estimates and a classical iteration scheme. Since $\left(p_{0}^{\varepsilon}, u_{0}^{\varepsilon}, F_{0}^{\varepsilon}\right) \in X_{N S}^{\varepsilon, m}$, there exists a sequence of smooth approximate initial data $\left(p_{0}^{\varepsilon, \delta}, u_{0}^{\varepsilon, \delta}, F_{0}^{\varepsilon, \delta}\right) \in$ $X_{N S, a p}^{\varepsilon, m}(\delta$ being a regularization parameter), which have enough spatial regularity so that the time derivatives at the initial time can be defined by Navier-Stokes equations and the boundary compatibility conditions are satisfied. We construct approximate solutions, inductively, as follows

(1) Define $u^{0}=u_{0}^{\varepsilon, \delta}$, and

(2) Assuming that $u^{k-1}$ was defined for $k \geq 1$, let $\left(\rho^{k}, u^{k}\right)$ be the unique solution to the following linearized initial boundary value problem:

$$
\left\{\begin{array}{l}
\rho_{t}^{k}+\operatorname{div}\left(\rho^{k} u^{k-1}\right)=0 \text { in }(0, T) \times \Omega_{t}, \\
\rho^{k} u_{t}^{k}+\rho^{k} u^{k-1} \cdot \nabla u^{k}+\nabla p^{k}=\varepsilon \Delta u^{k}+\varepsilon \nabla \operatorname{div} u^{k} \text { in }(0, T) \times \Omega_{t}, \\
\partial_{t} F^{k}+u^{k-1} \cdot \nabla F^{k}=0, \text { on } \partial \Omega_{t}, \\
\left.\left(\rho^{k}, u^{k}, F^{k}\right)\right|_{t=0}=\left(\rho_{0}^{\varepsilon, \delta}, u_{0}^{\varepsilon, \delta}, F_{0}^{\varepsilon, \delta}\right), \text { with } \frac{2}{3 C_{0}} \leq \rho_{0}^{\varepsilon, \delta} \leq \frac{3}{2} C_{0}, \\
\text { with boundary conditions }
\end{array}\right.
$$

Since $\rho^{k}, u^{k}$ and $F^{k}$ are decoupled, the existence of the global unique smooth solution $\left(\rho^{k}, u^{k}\right)$ of (5.2) with $0<\rho^{k}<\infty$ can be obtained by using known results. For example, one can first obtain a local existence result for (5.2) by using local existence result in [40, without surface tension and 63, 64] with surface tension. Since (5.2) is a linear problem, one can extend the local solution to a global one. Although the local existence result of 40 without surface tension and 63, 64 with surface tension is proved in Sobolev-Slobodetskii spaces $W^{l, \frac{l}{2}}$ (see [63]) with $l \geq 0$, but it is enough for us to proceed our $a$ priori estimates in Section 4 by taking $l=3 \mathrm{~m}$.

Similar to Section 1, let $\left(p^{k}, v^{k}, h^{k}\right)$ be the localized version $\left(p^{k}, u^{k}, F^{k}\right)$. Applying the a priori estimates given in Theorem 4.1 and by an induction argument, we obtain a uniform time $T_{1}>0$ and constant $\tilde{C}_{3}>0$ (independent of $\varepsilon, \sigma$ and $\delta$ ), such that it holds for $\left(p^{k}, v^{k}, h^{k}\right), k \geq 1$ that

$$
\Theta_{m}\left(p^{k}, v^{k}, h^{k}\right)(t) \leq \tilde{C}_{3}, \forall t \in\left[0, T_{1}\right]
$$

and

$$
\frac{1}{2 C_{0}} \leq \varrho^{k}(t) \leq 2 C_{0}, \quad \forall t \in\left[0, T_{1}\right]
$$

where $T_{1}$ and $\tilde{C}_{3}$ depend only on $C_{0}, \tilde{C}_{0}$ and $d_{0}$. Based on the above uniform estimates for $\left(\rho^{k}, u^{k}, F^{k}\right)$, using Lagrangian coordinates and considering the difference of $\left(\rho^{k+1}-\rho^{k}, u^{k+1}-u^{k}, F^{k+1}-F^{k}\right)$, one can 
prove, by using the energy methods, that there exists an uniform time $T_{2}\left(\leq T_{1}\right)$ (independent of $\varepsilon, \sigma$ and $\delta$ ) such that $\left(\rho^{k}, u^{k}, F^{k}\right)$ converges to a limit $\left(\rho^{\varepsilon, \delta}, u^{\varepsilon, \delta}, F^{\varepsilon, \delta}\right)$ as $k \rightarrow+\infty$ in the following strong sense:

$$
\left\{\begin{array}{l}
\left(\rho^{k}, u^{k}\right) \rightarrow\left(\rho^{\varepsilon, \delta}, u^{\varepsilon, \delta}\right) \text { in } L^{\infty}\left(0, T_{2} ; L^{2}\right) \\
\nabla u^{k} \rightarrow \nabla u^{\varepsilon, \delta} \text { in } L^{2}\left(0, T_{2}, L^{2}\right) \\
F^{k}(t, x(\cdot, \cdot)) \rightarrow F^{\varepsilon, \delta}(t, x(\cdot, \cdot)) \text { in } L^{2}(U) .
\end{array}\right.
$$

Then, it is easy to $\operatorname{check}\left(\rho^{\varepsilon, \delta}, u^{\varepsilon, \delta}, F^{\varepsilon, \delta}\right)$ is a weak solution to the problem (1.1), (1.5) and (1.6) with initial data $\left(\rho_{0}^{\varepsilon, \delta}, u_{0}^{\varepsilon, \delta}, F_{0}^{\varepsilon, \delta}\right)$. Then, by virtue of the lower semi-continuity of norms, one can deduce from the uniform bounds (5.3) and (5.4) that $\left(\rho^{\varepsilon, \delta}, u^{\varepsilon, \delta}, F^{\varepsilon, \delta}\right)$ satisfies the following regularity estimates

$$
\Theta_{m}\left(p^{\varepsilon, \delta}, v^{\varepsilon, \delta}, h^{\varepsilon, \delta}\right)(t) \leq \tilde{C}_{3}, \forall t \in\left[0, T_{2}\right]
$$

and

$$
\frac{1}{2 C_{0}} \leq \varrho^{\varepsilon, \delta}(t) \leq 2 C_{0}, \quad \forall t \in\left[0, T_{2}\right]
$$

Based on the uniform estimates (5.5) and (5.6) for $\left(\rho^{\varepsilon, \delta}, u^{\varepsilon, \delta}, F^{\varepsilon, \delta}\right)$, we pass the limit $\delta \rightarrow 0$ to get a strong solution $\left(\rho^{\varepsilon}, u^{\varepsilon}, F^{\varepsilon}\right)$ to (1.1), (1.5) and (1.6) with initial data $\left(\rho_{0}^{\varepsilon}, u_{0}^{\varepsilon}, F_{0}^{\varepsilon}\right)$ satisfying (5.1) by using a strong compactness arguments. Since the compactness arguments are almost the same as the ones need for the proof of Theorem 1.10 below, we shall not give more details here. Moreover, the uniform bounds (1.47) and (1.48) are immediately results of the lower semi-continuity of norms. Changing the variable into Lagrangian coordinate and using energy method, it is easy to prove the uniqueness of $\left(\rho^{\varepsilon}, u^{\varepsilon}, F^{\varepsilon}\right)$ since we work on functions with enough regularity. Taking $T_{0}=T_{2}$ and $\tilde{C}_{1}=\tilde{C}_{3}$, we complete the proof of Theorem 1.5

Proof of Theorem 1.10. For any fixed $\sigma \geq 0$, it follows from Theorem [1.5, for any $\varepsilon \in(0,1]$, that

$$
\Theta_{m}\left(p^{\varepsilon}, v^{\varepsilon}, h^{\varepsilon}\right)(t) \leq \tilde{C}_{1}, \forall t \in\left[0, T_{0}\right],
$$

and

$$
\frac{1}{2 C_{0}} \leq \varrho^{\varepsilon}(x, t) \leq 2 C_{0}, \quad \forall t \in\left[0, T_{0}\right]
$$

Thus, $\left(p^{\varepsilon}, v^{\varepsilon}\right)$ is uniform bounded in $L^{\infty}\left([0, T] ; H_{c o}^{m}\right), \nabla\left(p^{\varepsilon}, v^{\varepsilon}\right)$ is uniform bounded in $L^{\infty}\left([0, T] ; H_{c o}^{m-2}\right)$, $h^{\varepsilon}$ is uniform bounded in $L^{\infty}\left(0, T ; H^{m}\right), \partial_{t}\left(p^{\varepsilon}, v^{\varepsilon}\right)$ is uniform bounded in $L^{\infty}\left([0, T] ; L^{2}\right)$ and $\partial_{t} h^{\varepsilon}$ is uniform bounded in $L^{\infty}\left(0, T ; L^{2}\right)$. Then, it follows from the strong compactness arguments(see [42]) that $\left(p^{\varepsilon}, v^{\varepsilon}\right)$ is compact in $C\left([0, T] ; H_{c o}^{m-2}\right)$ and $h^{\varepsilon}$ is compact in $C\left([0, T] ; H^{m-1}\right)$. In particular, there exist a sequence $\varepsilon_{n} \rightarrow 0+$ and $(p, v) \in C\left([0, T] ; H_{c o}^{m-2}\right)$ and $h \in C\left([0, T] ; H^{m-1}\right)$ such that

$$
\left\{\begin{array}{l}
\left(p^{\varepsilon_{n}}, v^{\varepsilon_{n}}\right) \rightarrow(p, v) \text { in } C\left([0, T] ; H_{c o}^{m-2}\right), \\
h^{\varepsilon} \rightarrow h \text { in } C\left([0, T] ; H^{m-1}\right),
\end{array} \text { as } \varepsilon_{n} \rightarrow 0+,\right.
$$

Denote $(\rho, u, F)$ the global version of $(\varrho, v, h)$. Applying the lower semi-continuity of norms to the bounds (5.7) and (5.8), we obtain the uniform estimates (1.50) and (1.51). Then, based on (5.9) and the uniform estimates (1.50), (1.51), it is easy to check that $(\rho, u, F)$ is a solution of the Euler equations with the free surface

$$
\left\{\begin{array}{l}
\partial_{t} \rho+\operatorname{div}(\rho u)=0, \\
\rho \partial_{t} u+\rho(u \cdot \nabla) u+\nabla p=0,
\end{array} \quad x \in \Omega_{t}, t \in\left(0, T_{0}\right]\right.
$$

with the boundary conditions

$$
\partial_{t} F+u \cdot \nabla F=0, \quad \text { and } p=p_{e}-\sigma H, \quad x \in \partial \Omega_{t},
$$

where $\Omega_{t}$ is the domain occupied by the fluid on time $t \geq 0$ and the boundary of $\Omega_{t}$ is given by

$$
\partial \Omega_{t}=\left\{x \in \mathbb{R}^{3} \mid F(x, t)=0\right\} .
$$


By using (1.50), (5.9) and the anisotropic Sobolev inequality (4.1.3), we have that

$$
\sup _{t \in[0, T]}\left\|\left(\varrho^{\varepsilon_{n}}-\rho, v^{\varepsilon_{n}}-v\right)\right\|_{L^{\infty}}^{2} \leq \sup _{t \in[0, T]}\left(\left\|\nabla\left(\varrho^{\varepsilon_{n}}-\varrho, v^{\varepsilon_{n}}-v\right)\right\|_{H_{c o}^{1}} \cdot\left\|\left(\varrho^{\varepsilon_{n}}-\varrho, v^{\varepsilon_{n}}-v\right)\right\|_{H_{c o}^{2}}\right) \rightarrow 0 .
$$

and

$$
\left|h^{\varepsilon}-h\right|_{W^{1, \infty}} \leq\left|h^{\varepsilon}-h\right|_{H^{3}} \rightarrow 0,
$$

as $\varepsilon_{n} \rightarrow 0+$. Hence, (1.52) is proved.

Consider $\left(\rho^{i}, u^{i}, F^{i}\right), i=1,2$ two solutions of (5.10), (5.11) defined on $[0, T]$ with the same initial data and the regularity stated in (1.50) and (1.51). We shall prove that $\left(\rho^{1}, u^{1}, F^{1}\right)=\left(\rho^{2}, u^{2}, F^{2}\right)$. Applying the standard energy method to the difference $\left(\rho^{1}, u^{1}, F^{1}\right)-\left(\rho^{2}, u^{2}, F^{2}\right)$ in the Lagrangian coordinates and using the uniform bounds in (1.50) and (1.51). Therefore, $(\rho, u, F)$ is a unique solution to Euler system with free surface (5.10)-(5.11). And the uniqueness yields immediately that the whole family $\left(\rho^{\varepsilon}, u^{\varepsilon}, F^{\varepsilon}\right)$ converges to $(\rho, u, F)$. Thus, the proof Theorem 1.10 is completed.

Proof of Theorem 1.11 Based on the uniform in both $\varepsilon$ and $\sigma$ estimates in Theorem 1.5, one can prove Theorem 1.11 by using the similar strong compactness argument in Theorem 1.10 The details are omitted here for brevity.

\section{Appendix}

Let $S(t, \tau)$ be the $C^{0}$ evolution operator generated by the following equation

$$
\left[\partial_{t} h+b_{1}(t, y) \partial_{y^{1}} h+b_{2}(t, y) \partial_{y^{2}} h+z b_{3}(t, y) \partial_{z} h\right]-\varepsilon d(t, y) \partial_{z z} h=0, z>0, t>\tau,
$$

with the boundary condition $h(t, y, 0)=0$ and with the initial condition $h(\tau, y, z)=h_{0}(y, z)$. The coefficients are smooth and $d(t, y)$ satisfies

$$
c_{1} \leq d(t, y) \leq \frac{1}{c_{1}}
$$

for some positive constant $c_{1}>0$.

Then we have the following Lemnas whose proof can be found in [53, the details are omitted here.

Lemma 6.1 It holds that, for $t \geq \tau \geq 0$

$$
\begin{aligned}
& \left\|S(t, \tau) h_{0}\right\|_{L^{\infty}} \leq\left\|h_{0}\right\|_{L^{\infty}}, \\
& \left\|z \partial_{z} S(t, \tau) h_{0}\right\|_{L^{\infty}} \leq C\left(\left\|h_{0}\right\|_{L^{\infty}}+\left\|z \partial_{z} h_{0}\right\|_{L^{\infty}}\right),
\end{aligned}
$$

where $C>0$ is a uniform constant independent of the bound of $d(t, y)$ and $b_{j}(t, y), j=1,2,3$.

Lemma 6.2 Consider h a smooth solution of

$$
a(t, y)\left[\partial_{t} h+b_{1}(t, y) \partial_{y^{1}} h+b_{2}(t, y) \partial_{y^{2}} h+z b_{3}(t, y) \partial_{z} h\right]-\varepsilon \partial_{z z} h=G, z>0, h(t, y, 0)=0,
$$

for some smooth function a $(t, y)$ satisfies (6.2) and vector fields $b=\left(b_{1}, b_{2}, b_{3}\right)^{t}(t, y)$. Assume that $h$ and $G$ are compactly supported in $z$. Then, one has the estimate:

$$
\begin{aligned}
\|h\|_{\mathcal{H}^{1, \infty}} \lesssim & \left\|h_{0}\right\|_{\mathcal{H}^{1, \infty}}+\int_{0}^{t}\left\|\frac{1}{a}\right\|_{L^{\infty}}\|G\|_{\mathcal{H}^{1, \infty}} d \tau \\
& +\int_{0}^{t}\left(1+\left\|\frac{1}{a}\right\|_{L^{\infty}}\right)\left(1+\sum_{i=0}^{2}\left\|Z_{i}(a, b)\right\|_{L^{\infty}}^{2}\right)\|h\|_{\mathcal{H}^{1, \infty}} d \tau .
\end{aligned}
$$

\section{Acknowledgments:}

Yong Wang is partially supported by National Natural Sciences Foundation of China No. 11371064 and 11401565. This research is support in part by Zheng Ge Ru Funds, Hong Kong RGC Earmarked Research Grants CUHK4041/11P, and CUHK4048/13P, NSFC/RGC Joint Research Scheme Grant N. CUHK443/14, A grant from the Croucher Foundation, and a Focus Area Grant at The Chinese University of Hong Kong. 


\section{References}

[1] S. Alinhac. Existence d'ondes de rarfaction pour des systmes quasi-linaires hyperboliques multidimensionnels. (French) [Existence of rarefaction waves for multidimensional hyperbolic quasilinear systems] Comm. Partial Differential Equations 14, no. 2, 173-230, 1989.

[2] D. M. Ambrose, N. Masmoudi. The zero surface tension limit of two-dimensional water waves. Comm. Pure Appl. Math., 58(10), 1287-1315, 2005.

[3] D. M. Ambrose, N. Masmoudi. The zero surface tension limit of three-dimensional water waves. Indiana Univ. Math. J., 58(2):479-521, 2009.

[4] J. T. Beale. Large-time regularity of viscous surface waves. Arch. Rational Mech. Anal., 84(4):307$352,1983 / 84$.

[5] J. T. Beale, T. Hou, J. Lowengrub. Growth rates for the linearized motion of fluid interfaces away from equilibrium. Comm. Pure Appl. Math., 46, 1269-1301, 1993.

[6] H. Beirao da Veiga, F. Crispo, Sharp inviscid limit results under Navier type boundary conditions, An $L^{p}$ theory. J. Math. Fluid Mech. 12, 397-411, 2010.

[7] H. Beirao da Veiga, F. Crispo, Concerning the $W^{k, p}$-inviscid limit for $3 D$ flows under a slip boundary condition. J. Math. Fluid Mech. 13, 117-135, 2011.

[8] Y. G. Cho, H. J. Choe, H. Kim, Unique solvability of the initial boundary value problems for compressible viscous fluids. J. Math. Pures Appl. 83, no. 2, 243-275, 2004.

[9] P. Constantin, Note on loss of regularity for solutions of the 3-D incompressible Euler and related equations. Comm. Math. Phys. 104, 311-326, 1986.

[10] P. Constantin, C. Foias, Navier Stokes equation. Univ. of Chicago press IL, 1988.

[11] W. Craig. An existence theory for water waves and the Boussinesq and Korteweg-de Vries scaling limits. Comm. Partial Differential Equations, 10 787-1003, 1985.

[12] D. Christodoulou and H. Lindblad. On the motion of the free surface of a liquid. Comm. Pure Appl. Math., 53(12), 1536-1602, 2000.

[13] D. Coutand, J. Hole, S. Shkoller, Well-posedness of the free-boundary compressible 3-D Euler equations with surface tension and the zero surface tension limit. SIAM J. Math. Anal. 45, no. 6, 3690-3767, 2013.

[14] D. Coutand, S. Shkoller, Well-posedness of the free-surface incompressible Euler equations with or without surface tension, J. Amer. Math. Soc., 20, 829-930, 2007.

[15] G. M. Gie, J. P. Kelliher, Boundary layer analysis of the Navier-Stokes equations with generalized Navier boundary conditions. J. Differential Equations. 253, no. 6, 1862-1892, 2012

[16] T. Elgindi, D. Lee, Uniform regularity for free-boundary Navier-Stokes equations with surface tension, arXiv:1403.0980

[17] P. Germain, N. Masmoudi, J. Shatah. Global solutions for the gravity water waves equation in dimension-3. C. R. Math. Acad. Sci. Paris, 347(15-16), 897-902, 2009.

[18] P. Germain, N. Masmoudi, J. Shatah. Global solutions for the gravity water waves equation in dimension 3. Ann. of Math. (2) 175, no. 2, 691-754, 2012.

[19] O. Gues, Probleme mixte hyperbolique quasi-lineaire caracteristique. Comm. Partial Differential Equations. 15, no. 5, 595-645, 1990

[20] Y. Guo, I. Tice, Local well-posedness of the viscous surface wave problem without surface tension. Anal. PDE 6, no. 2, 287-369, 2013. 
[21] D. Iftimie, F. Sueur, Viscous boundary layers for the navier-stokes equations with the navier slip conditions. Arch. Rat. Mech. Analy., 199, no.1, 145-175, 2011.

[22] T. Kato, Nonstationary flows of viscous and ideal fluids in $\mathbb{R}^{3}$. J. Functional Analysis 9, 296-305, 1972.

[23] T. Kato, Remarks on zero viscosity limit for non-stationary Navier-Stokes flows with boundary. In: Seminar on PDE (S.S.Chen, eds), Springer, New York, 85-98, 1984.

[24] D. Lannes. Well-posedness of the water-waves equations. J. Amer. Math. Soc., 18(3), 605-654, 2005.

[25] H. Lindblad, Well-posedness for the motion of an incompressible liquid with free surface boundary. Ann. of Math. (2), 162(1), 109-194, 2005.

[26] H. Lindblad, Well posedness for the motion of a compressible liquid with free surface boundary. Comm. Math. Phys., 260, 319-392, 2005.

[27] Y. Maekawa, On the inviscid limit problem of the vorticity equations for viscous incompressible flows in the half plane. Comm. Pure Appl. Math. 67 no. 7, 1045-1128, (2014).

[28] A. J. Majda, A. L. Bertozzi, Vorticity and incompressible flow. Cambridge Texts in Applied Mathematics 27. Cambridge University Press, Cambridge, 2002.

[29] A. J. Majda, S. Osher, Initial-boundary value problems for hyperbolic equations with uniformly characteristic boundary. Comm. Pure. Appl. Math. 28, no. 5, 607-675, 1975.

[30] N. Masmoudi, Remarks about the inviscid limit of the Navier-Stokes system. Comm. Math. Phys. 270, no. 3, 777-788, 2007.

[31] N. Masmoudi, F. Rousset, Uniform Regularity for the Navier-Stokes equation with Navier boundary condition. Arch. Ration. Mech. Anal. 203, no. 2, 529-575, 2012.

[32] N. Masmoudi, F. Rousset, Uniform Regulartiy and Vanishing Viscosity Limit for the Free Surface Navier-Stokes Equations. arXiv:1202.0657

[33] C.L.M.H. Navier, Sur les lois de lequilibre et du mouvement des corps elastiques. Mem. Acad. R. Sci. Inst. France 6, 369, 1827.

[34] M. Ogawa and A. Tani. Free boundary problem for an incompressible ideal fluid with surface tension. Math. Models Methods Appl. Sci., 12, 1725-1740, 2002.

[35] M. Paddick, The strong inviscid limit of the isentropic compressible Navier-Stokes equations with Navier boundary conditions. arXiv:1410.2811v1.

[36] L. Rayleigh, On the instability of jets. Proc. London Math. Soc., s1-10, 4-13, 1878.

[37] M. Sammartino, R. E. Caflisch, Zero viscosity limit for analytic solutions of the Navier-Stokes equation on a half-space. I. Existence for Euler and Prandtl equations. Comm. Math. Phys. 192, no. 2, 433-461, 1998.

[38] M. Sammartino, R. E. Caflisch, Zero viscosity limit for analytic solutions of the Navier-Stokes equation on a half-space. II. Construction of the Navier-Stokes solution. Comm. Math. Phys. 192, no. 2, 463-491, 1998 .

[39] G. Schneider and C. E. Wayne. The rigorous approximation of long-wavelength capillary-gravity waves. Arch. Ration. Mech. Anal., 162(3), 247-285, 2002.

[40] P. Secchi, A. Valli, A free boundary problem for compressible viscous fluids. J. Reine Angew. Math, 341, 1-31, 1983.

[41] J. Shatah and C. Zeng. Geometry and a priori estimates for free boundary problems of the Euler equation. Comm. Pure Appl. Math., 61(5), 698-744, 2008.

[42] J. Simon, Compact sets in the space $L^{p}(0, T ; B)$. Ann. Mat. Pura Appl. 146, no. 4, 65-96, 1987. 
[43] V.A. Solonnikov. Solvability of a problem on the motion of a viscous incompressible fluid bounded by a free surface. Math. U.S.S.R. Izvestijya 11, 1323-1358, 1977.

[44] V. A. Solonnikov, A. Tani, Free boundary problem for a viscous compressible flow with surface tension. Zip. Nauchn. Sem. LOMI, 182 (1990), 142-148; also in Constantin Caratheodory: an International Tribute, M. Rassias, ed., Vol. 2, World Scientific, 1270-1303, 1991.

[45] G. Taylor, The instability of liquid surfaces when accelerated in a direction perpendicular to their planes I. Proc. Roy. Soc. London. Ser. A., 201, 192-196, 1950.

[46] Z. Tan, Y. Wang Zero surface tension limit of viscous surface waves. Comm. Math. Phys.,328, 733-807, 2014.

[47] N. Tanaka, A. Tani, Surface waves for a compressible viscous fluid. J. Math. Fluid Mech. 5, no. 4, 303-363, 2003.

[48] R. Teman, Navier-Stokes Equations: Theory and Numerical Analysis. New York, Oxford, 1979.

[49] A. Tani. Small-time existence for the three-dimensional Navier-Stokes equations for an incompressible fluid with a free surface. Arch. Rational Mech. Anal., 133(4):299-331, 1996.

[50] Y. Trakhinin, Local existence for the free boundary problem for the non-relativistic and relativistic compressible Euler equations with a vacuum boundary condition Comm. Pure Appl. Math, 62, 1551-1594, 2009.

[51] Y. G. Wang, M. Williams, The inviscid limit and stability of characteristic boundary layers for the compressible Navier-Stokes equations with Navier-friction boundary conditions Ann. Inst. Fourier (Grenoble) 62, no. 6, 2257-2314, 2013.

[52] Y. J. Wang, Z. P. Xin, Vanishing viscosity and surface tension limits of incompressible viscous surface waves. arXiv:1504.00152.

[53] Y. Wang, Z. P. Xin, Y. Yong, Uniform regularity and vanishing viscosity limit for the compressible Navier-Stokes with general Navier-slip boundary conditions in 3-dimensional domain. arXiv: $1501.01718 \mathrm{v} 1$.

[54] S. Wu. Well-posedness in Sobolev spaces of the full water wave problem in 2-D. Invent. Math., 130, 39-72, 1997.

[55] S. Wu. Well-posedness in Sobolev spaces of the full water wave problem in 3-D. J. Amer. Math. Soc., 12, 445-495, 1999.

[56] S. Wu. Almost global wellposedness of the 2-D full water wave problem. Invent. Math., 177, 45-135, 2009.

[57] S. Wu. Global wellposedness of the 3-D full water wave problem. Invent. Math., 184, 125-220, 2011.

[58] Y. L. Xiao, Z. P. Xin, On the vanishing viscosity limit for the 3D Navier-Stokes equations with a slip boundary condition. Comm. Pure Appl. Math. Vol. LX, 1027-1055, 2007.

[59] Y. L. Xiao, Z. P. Xin, On the Inviscid Limit of the 3D Navier-Stokes Equations with Generalized Navier-slip Boundary Conditions. Commun. Math. Stat. 1, no. 3, 259-279, 2013.

[60] Y. L. Xiao, Z. P. Xin, On 3D Lagrangian Navier-Stokes a Model with a Class of Vorticity-Slip Boundary Conditions. J. Math. Fluid Mech. 15, no. 2, 215-247, 2013.

[61] Z. P. Xin, T. Yanagisawa Zero-viscosity limit of the linearized navier-stokes equations for a compressible viscous fluid in the half-plane. Comm. Pure Appl. Math., 52, 479-541, 1999.

[62] H. Yosihara, Gravity waves on the free surface of an incompressible perfect fluid of finite depth. Publ. Res. Inst. Math. Sci., 18(1):49-96, 1982.

[63] W. M. Zajaczkowski, Existence of local solutions for free boundary problems for viscous compressible barotropic fluids. Ann. Polon. Math. 60, no. 3, 255-287, 1995. 
[64] W. M. Zajaczkowski, On nonstationary motion of a compressible barotropic viscous capillary fluid bounded by a free surface. SIAM J. Math. Anal. 25, no. 1, 1-84, 1994.

[65] P. Zhang and Z. Zhang. On the free boundary problem of three-dimensional incompressible Euler equations. Comm. Pure Appl. Math., 61(7), 877-940, 2008. 